


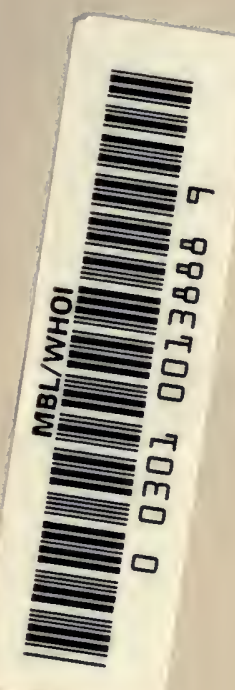




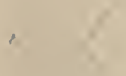






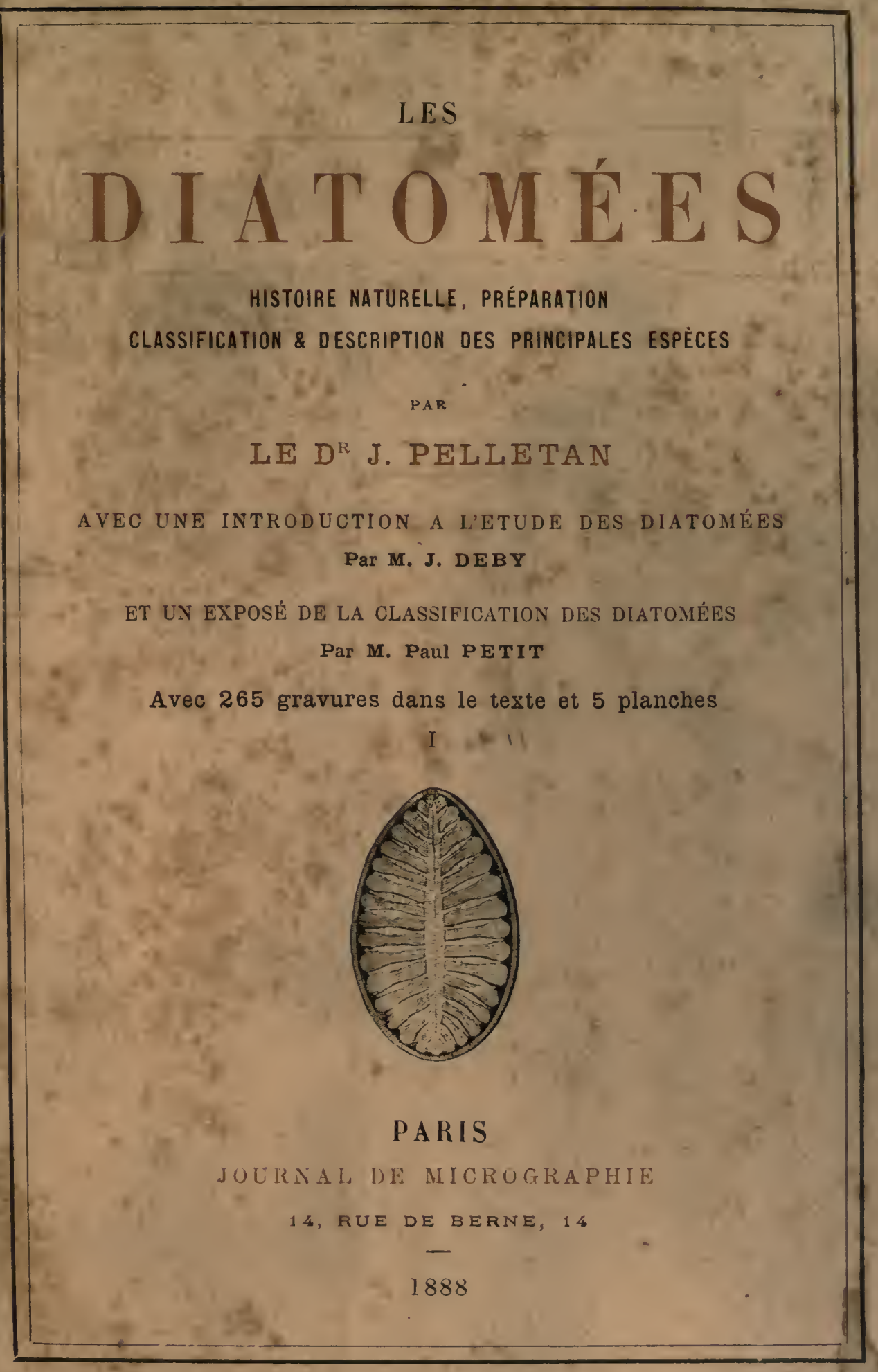


L ES

\section{I A T O M É E S}




\section{PARIS}

IMPRIMERIE TYPOGRAPHIQUE J. BOLBACH 23, RUE DE LILLE, 25 


\section{LES}

\section{I A T O M É E S}

HISTOIRE NATURELLE, PRÉPARATION

CLASSIFICATION \& DESCRIPTION DES PRINCIPALES ESPĖCES

$$
\text { PAR }
$$

\section{LE $D^{R}$ J. PELLETAN}

AVEC UNE INTRODUCTION A L'ETUDE DES DIATOMEES Par M. J. DEBY

- ET UN EXPOSÉ DE la CLASSIFICATION DES DIAT́OMÉES Par M. Paul PETIT

Avec 265 gravures dans le texte et 5 planches I
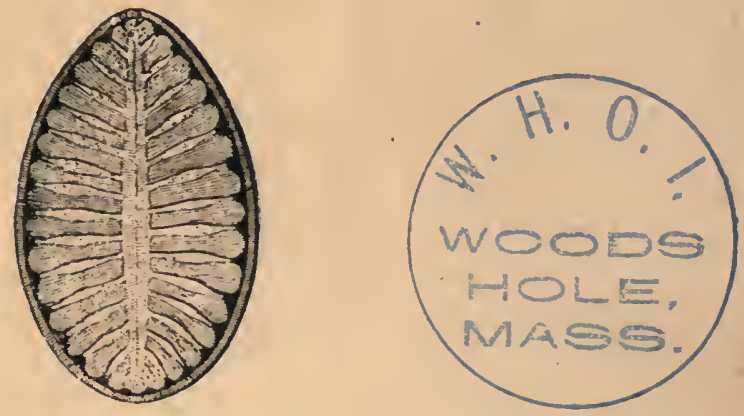

PARIS

JOURNAL DE MICROGRAPHIE 14, RUE DE BERNE, 14 



\section{TABLE}

\section{SYSTÉMATIQUE DES MATIERES}

PAGFS

Préface.

\section{PREMIERE PARTIE}

Introduction, par M. J. DebY . . . . . . . . . . . . 1

Déduplication des Diatomées . . . . . . . . . 3

Mouvements des Diatomées . . . . . . . . . . 3

Structure microscopique des Diatomées . . . . . 6

Reproduction des Diatomés. . . . . . . . 7

\section{CHAPITRE I}

Historique. . . . . . . . . . . . . . . 11

\section{CHAPITRE II}

1. Histoire naturelle des Diatomées . . . . . . 20

2. Mourements des Diatomées. . . . . . . . . 31

3. Multiplication des Diatomées . . . . . . . . 36

4. Mode de végétation des Diatomćes . . . . . . . 52

5. Réviviscence des Diatomées ... . . . . . . 55 
Structure des Diatomées . . . . . . . . . . . . 57

1. Valves . . . . . . . . . . . . . 57

2. Structure intime des valves. . . . . . . . . . . . 66

3. Appendices et dispositions particulieres. . . . . . . . 84

\section{CHAPITRE IV}

Recherche et RÉcolte des Diatonées. . . . . . . . . 93

1. Les Diatomées dans la nature . . . . . . . . . 93

2. Récolte des Diatomées . . . . . . . . . . . . 99

CHAPITRE V

Technique des Diatomées. . . . . . . . . . . . 106

1. Nettoyage des Diatomées. . . . . . . . . . 106

2. Triage des Diatomées . . . . . . . . . . , 114

CHAPITRE VI

Montage Des Diatomées. . . . . . . . . . . . 118

1. Instruments et produits. . . . . . . . . . . 118

2. Montage à sec. . . . . . . . . . . . . 122

3. Montage dans les baumes. . . . . . . . . . 124

4. Montage dans les liquides. . . . . . . . . . . 127

5. Préparations systématiques. . . . . . . . . 128

\section{CHAPITRE VII}

MOYens D'ĖTUde . . . . . . . . . . . . . . . . . . 138

1. Les Microscopes. . . . . . . . . . . . 138

2. Les Objectifs . . . . . . . . . . . . . . 148

3. Condensateurs et Appareils divers : . . . . . . . . 164

4. Éclairage . . . . . . . . . . . . . . 175

5. Microtomes.............. . 180

CHAPITRE VIII

Classification . . . . . . . . . . . . . . 188

1. Systèmes de classiflcation. . . . . . . . 188

2. Classification par M. Paul Petit. . . . . . . . 184 
Placochromaticées. HaGes

Placochromaticées. - Achnanthées. . . . . . . . 192

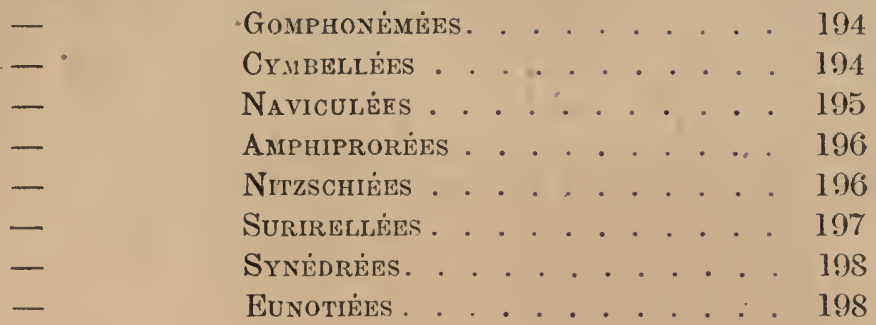

Coccochromaticées. - Fragilariées. . . . . . . . . 199

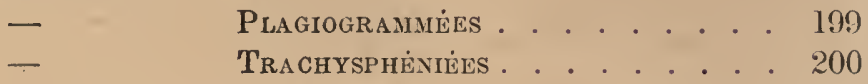

— Lrcmophoríes . . . . . . 201

— Tabellariées. . . . . . . 201

- Rhizosolíniées. . . . . . 202

- Chatocérées . . . . . . . 203

- BiddulphíEes. . . . . . . . 204

— Eupodiscées . . . . . . 201

- Héliopeltées. . . . . . . . 205

Astérolamprées . . . . . . . . 206

Coscinodiscées. . . . . . . . 206

Xanthiopyxidées. . . . . . . 207

Gathlonellées . . . . . . . 208

\title{
DEUXIEME PARTIE
}

\author{
CHA PITRE $1 \mathrm{X}$
}

Description Des PRINCIPALES ESPÈCES. . . . . . . . . 211

1. - Jre tribu : Achnanthées. . . . . . . . . 211

2. - $2^{\circ}$ tribu : Gomphonémées. . . . . . . . . 218

3. - $3^{\circ}$ tribu : Cymbellées. . . . . . . . . $225^{\circ}$

CHAPITRE X

Description des principales espèces $(S u \imath t e) . . . . . . .2243$

1. - $4^{0}$ tribu : Naviculées ............. 243

2. - Genre Navicula ........... 245 


\section{CHAPITRE XI}

Description des principales espèces (Suite). . rags

1. - $4^{\mathrm{e}}$ tribu : Naviculées (suite), G. Stauroneis. . . . . 282

2. - - G. Scoliopleura. . . 287

3. -. - $\quad$ G. Pleurosigma. . . 294

4. - - G. Donkinia, Toxonidea, Amphipleura, etc. . . . . . . . . . . . 306

Page 197, ligne 11. - C'est par erreur que le genre Amphiprora, qui appartient à la famille des AMphiprorḱes est répété parmi ceux qui composent la famille des NrtzschiÉes. Il faut le supprimer à cet endroit. 


\section{PREFACE}

Nous n'avons pas eu, en publiant cet ouvrage, l'intention de faire une monographie complète de la famille des Diatomées, rapportant tout ce qui a été dit, écrit ou imaginé sur ces charmantes Algues; nous avons voulu seulement présenter un résumé de leur histoire, un tableau général de leur organisation, de leurs caractères, de leur classification, avec la description des espèces que l'on rencontre le plus fréquemment. 'Néanmoins, sans nous égarer dans les questions de détail et les discussions de systématique, nous avons cherché à donner à notre travail assez de développement pour qu'il pût èveiller chez nos lecteurs le désir d'aller plus loin, d'étudier plus á fond ces intéressants organismes, et, par exemple, de résoudre les problèmes, si nombreux encore, qu'ils présentent à la sagacité, à la patience et à l'habileté des micrographes.

C'est pour atteindre plus sûrement notre but que nous avnns tenu a mettre notre livre, pour ainsi dire, sous le patronage de deux des diatomistes les plus autorisés de notre époque, qui, non seulement nous ont aidé des conseils de leur longue expérience, mais encore ont bien voulu nous prêter le concours de leur plume: M. Julien Deby, de Londres, qui a accepté la tâche de présenter notre ouvrage au public et d'en écrire l'Introduction, et M. Paul Petit, de Paris, qui s'est chargé de rédiger le chapitre relatif à la Classification des Diatomées.

Nous prions les deux savants auteurs de recevoir ici tous nos plus sincères et nos plus vifs remerciements pour leur amicale assistance et leur précieuse collaboration. 
D'autre part, dans la description de plusieurs centaines d'espèces qui constitue la deuxième partie de ce livre, c'est particulièrement au $D^{r} \mathrm{H}$. Van Heurck, d'Anvers, que nous nous sommes adressé; c'est de sa méthode descriptive que nous nous sommes généralement inspiré, telle qu'il l'a instituée dans son bel ouvrage que tout le monde connait, la Synopsis des Diatomées de Belgique; ce sont ses diagnoses et ses figures que nous avons le plus souvent suivies, comme ce sont ses chiffres que nous avons presque toujours adoptés pour les nombres de stries et autres mensurations délicates, chiffres qui, toutes les fois que nous avons eu à les contrôler, nous ont toujours paru remarquablement exacts.

Pour le reste, c'est à MM. Paul Petit, J. Deby, A. Truan, de Gijon; J. Brun, de Genève; P.-T Clève, d'Upsal; A. Grunow, de Vienne; J. Pantocsek, de Tavarnok, et divers autres auteurs, ou à nos observations particulières que nous avons emprunté les documents dont nous avons eu besoin.

Ce n'est pas à dire pour cela que, devant l'autorité de ces savants spécialistes, nous ayons renoncé à exprimer les idées personnelles que nous nous sommes faites sur beaucoup de points. Loin de là. Sur un grand nombre de questions discutées, nous avons émis des opinions qui nous sont propres, et nous ne nous dissimulons pas que, dans bien des cas, elles ne seront pas facilement admises par tous les diatomistes, à qui souvent elles ne paraitront guère orthodoxes. Nous les avons exprimées néanmoins, et nous les maintenons jusqu'à plus ample informé, par cette raison toute simple que nous les croyons justes, fondées d'ailleurs sur des observations directes, faites avec le plus grand soin, dans les meilleures conditions et surtout en dehors de toute idée préconçue. Nous laissons au temps le soin de les confirmer, et si, au contraire, il les renverse, elles auront encore servi à quelque chose puisqu'elles auront fait travailler.

Nous avons divisé notre ouvrage en trois parties.

Dans la première, après un très court historique de la découverte des Diatomées et des traraux dont clles ont été l'objet de la part des observateurs de la fin du siècle dernier et du commencement de celui-ci, nous étudions ces organismes au point de vue 
botanique et au point de vue micrographique. Puis nous indiquons les procédés de leur récolte, les localités on ils se trouvent, les méthodes que l'on met en œuvre pour les préparer à l'examen microscopique; enfin, les meilleurs instruments que l'on peut employer pour les étudier. Cette première partie se termine par l'exposé de leur classification par M. Paul Petit.

La seconde est tout entière consacrée à la description des principales espèces. On comprend que dans un ouvrage que nous voulions faire " élémentaire " et dont le cadre était, par conséquent, limité, nous ne pouvions songer à signaler, et encore moins à décrire, chacune des dix à douze mille espèces connues, et dont le nombre augmente d'ailleurs tous les jours. Nous ne pouvions même pas décrire tous les genres, bien que la classification de M. Paul Petit, que nous avons adoptée, en ait notablement réduit la liste. Nous avons nécessairement dû faire un choix, et nous nous sommes arrêté aux genres et aux espèces le plus généralement répandus, à ceux que l'on rencontre le plus communément dans les mers, les cours d'eaux ou les dépôts de nos pays et des contrées voisines, de préférence aux genres et aux espèces absolument exotiques, que l'on ne trouve que dans les parages lointains, ou exclusivement localisés dans certains dépôts, certains fleuves, certaines mers, disséminés en différents points du globe.

Sans doute, cette nécessité de nous restreindre nous a forcé de passer sous silence bien des espèces remarquables et intéressantes; mais, si nous eussions voulu les faire figurer dans notre livre, il aurait rapidement acquis l'étendue de plusieurs in-folios. Nous sommes donc obligé de renvoyer nos lecteurs, pour l'étude de ces espèces, aux grands ouvrages spéciaux et aux monographies particulières qui ont été publiées sur les Diatomées des différentes localités, nous bornant à signaler, lorsque cela nous a été possible, quelques-unes des formes les plus typiques.

C'est pour remédier, autant qu'il était en notre pouvoir, à l'exiguïté de notre cadre que nous avons donné quelque étendue à notre troisième partie, entièrement consacrée à une Bibliographie aussi complète que nous avons pu la faire, et dans laquelle nos lecteurs trouveront l'indication de nombreux matériaux pour parfaire leur instruction diatomologique. 
Nous avons néanmoins donné la description de cinq à six cents espèces, choisies dans toutes les tribus de la famille des Diatomées; de plus, au lieu de les énumérer les unes après les autres, comme les articles d'un cataloguc, ou de les distribuer empiriquement d'après la mèthode dichotomique, qui est parfois très difficile à appliquer et établit souvent des divisions fort peu logiques, nous avons suivi un autre système que nous croyons meilleur, du moins dans un ouvrage tel que le nôtre. Nous avons, dans chaque genre, choisi un ou plusieurs types les plus caractérisés, autour desquels nous avons groupé les espèces qui leur ressemblent le plus, en indiquant les détails qui les rapprochent et ceux qui les distinguent les unes des autres et du type commun. Ce système, peut-ètre moins méthodique, est infiniment moins fastidieux pour le lecteur et permet de décrire le plus d'objets dans le moins de lignes.

Nous avons, en effet, écrit pour des " lecteurs " lisant cet ouvrage comme on lit un livre quelconqne traitant d'histoire naturelle, et non pour des savants consultant un recueil de documents scientifiques. C'est pourquoi nous avons adopté une forme tout à fait nouvelle pour les ouvrages sur les Diatomées, c'est-àdire que nous avons renoncé au système des Atlas et des Planches, et inséré des gravures dans le texte, aux passages où elles l'expliquent ou le complètent. Les planches que l'on réunit à la fin du volume ou en atlas séparé, et où l'on peut grouper un grand nombre de figures, sont plus commodes pour l'auteur; mais si elles permettent, grâce aux procédés délicats de la lithographie, de donner des figures plus fines et plus artistiques, pouvant servir, comme nous le disions, de documents authentiques pour les savants qui les consultent plus que le texte, elles ne sont pas d'un maniement facile pour les lecteurs ordinaires, qui ont besoin d'avoir à la fois les figures et le texte sous les yeux. Il est rrai qu intercalées dans le texte, les figures typographiques ne peurent être que des reproductions par la gravure ou l'héliogravure de dessins ou de lithographies, que le tirage rapide des imprimeries ne permet guère de leur conserver toute la délicatesse et le fini du modèle; mais, telles qu'elles sont, nous avons pensé qu'elles suffisent au but que nous nous proposons, d'autant plus qu'elles nous paraissent racheter amplement ces défauts par l'avantage qu'elles ont de se trouver toujours 
à l'endroit utile, et enfin parce qu'elles permettent de faire des livres dont le prix est facilement abordable pour tout le monde.

Si donc nos lecteurs trouvent quelques-unes de nos gravures un peu défectueuses au point de vue des détails, nous les prions d'être indulgents, en raison de la difficulté que nous avions à vaincre. On sait combien infiniment petits sont sourent les dessins qui ornent les valves des Diatomées; obligé que nous étions encore de réduire la dimension de nos gravures pour les faire entrer dans le format d'un in-octavo, on comprend que certaines de ces gravures sont venues un peu confuses lorsqu'il s'agissait d'objets très fins, et d'autant plus qu'aucune n'existait, qu'il a fallu les créer toutes, notre ouvrage étant le premier qui ait paru sous cette forme dans la littérature scientifique de langue française.

Ceci dit, il ne nous reste plus qu'à livrer notre travail à l'appréciation du public et à le prier de vouloir bien, en raison de l'intention, excuser les fautes de l'auteur.

\section{Dr J. Pelletan}



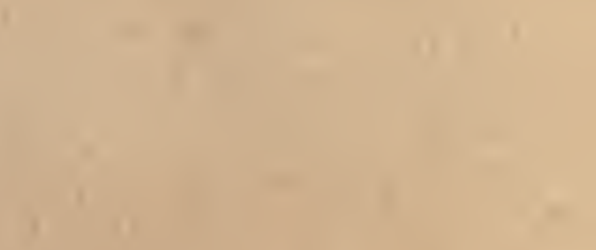


\section{PREMIÉRE PARTIE}

\section{HISTOIRE NATURELLE - PRÉPARATION}

\section{CLASSIFICATION}





\section{INTRODUCTION}

Partout dans ce monde où il existe de l'eau, ou même de l'humidité, la vie se manifeste avec vigueur par le développement d'organismes divers dont la multiplicité merveilleuse des formes paraît presqu'inépuisable. En majorité, ces êtres ont une fort petite taille: ce sont le plus souvent des "microscopiques que l'œil humain ne distingue pas sans l'emploi de la lentille grossissante. On a rangé les uns parmi les protozoaires ou animaux, les autres parmi les algues ou plantes aquatiques inférieures, c'est à dire qu'on les a partagés entre le règne animal et le rẻgne végétal. Ces deux grandes divisions ou règnes de la nature se confondent dans la région des infiniments petits et leur ligne de démarcation échappe alors au naturaliste, non pas à cause de la difficulté de la définir, mais bien plutòt parce que cette distinclion n'existe réellement pas dans la nature. Un troisième règne, celui des "protistes " a été, dans ces derniers temps, intercalé entre les précédents pour recevoir une foule de ces organismes douteux qui sont, à proprement parler, des animaux et des plantes, mais ce subterfuge, où la science veut se sauver par un mot, n'a pas de base scientitique solide et les êtres compris dans cette catégorie ne présentent pas de caractères spécifiques communs qui les différencient des autres produits vivants qui peuplent notre globe.

Parmi ces déclassés l'on rencontre, en nombre très considérable; une série de petites formes dont l'enveloppe ou carapace est formée en majeure partie de silice et auxquelles on a donné le nom de Bacillariées ou de Diatomées.

La beauté de sculpture, la variété et l'élégance des formes de ces petits êtres a depuis longtemps attiré l'attention des naturalistes et poussé les micrographes à les étudier avec assiduité, mais sans qu'on soit arrivé cependant, à leur égard, à des résultats scientifiques satisfaisants, surtout en ce qui concerne leur biologie.

Contrairement à l'opinion de tous les naturalistes qui m'ont précédé, et contrairement même aux vues que j'ai exprimées dans des publications antérieures, je suis arrivé actuellement à la conclusion que les Diatomées doivent être considérées, non pas comme des plantes uni-cellulaires, 
mais bien plutôt comme des algues pluri-cellulaires. Pour un grand nombre de familles et de genres de Diatomées cela me paraît parfaitement clair, manifeste et démontrable par le simple examen d'une longue série de formes linéaires telles que les Himanthidium, les Fragilaria, les Achnanthes, les Rhabdonema, les Striatella, les Melosira et beaut coup d'autres.

Ces plantes croissent comme le font toutes les autres algues simples et filamenteuses, par la division transverse successive des cellules végétatives (frustules, cliez les Diatomées) qui les constituent. Cette division des cellules végétatives continue pendant un temps plus ou moins long, puis tout à coup elle cesse et il se développe alors, aux dépens d'une ou de plusieurs des cellules végétatives, des fruits'ou sporanges destinés à la reproduction de l'espèce. Dans ces Diatomées chaque cellule végétative est adhérente d'une façon permanente à ses voisines.

Ėn dehor's de ces Diatomées filamenteuses, il existe une multitude d'autres espéces, qui ne présentent pas ce caractère de continuité, pour cette raison que les cellules végétatives de la plante ou les frustules, au lieu de rester adhérents, se sont successivement détachés l'un de l'autre a clraque division ou déduplication des cellules, et ont mené une vie indépendante. A la longue, cependant, après une succession de cellules purement végélatives, de frustules fo més par déduplication, il arrive un moment où une ou plusieurs de ces cellules végétatives produisent un fruit ou sporange tout comme cela a eu lieu pour les formes tilamienteuses.

Un examen quelque peu attentil des phénomènes de la vie et de la croissance des Diatomées nous conduit à considérer les frustules des Diatomées qu'on rencontre si fréquemment libres et isolés, comme étant, dans l'immense majorité des cas, de simples cellules végétatives destinées à périr sans se reproduire, après avoir donné le jour à d'autres générations de cellules végétatives dont un nombre trẻs infinitésimal seulement donnera naissance à des sporanges reproducteurs. Les centaines de millions de frustules qu'on rencontre partout oủ on les cherche no sont donc pas des individualités, ou des plantes complètes, comme on l'a cru ; ce sont les parties élémentaires à vie végétative d'un thalle qu'on pourrait, à défaut d'un terme plus convenable, appeler un thalle diffluent. D’après notre manière de voir, toutes les Diatomées sont partageables en deux séries : $1^{\circ}$ les Diatomées filamenteuses proprement dites el $2^{\circ}$ les Dialomées à frustules libres. Les unes comme les autres sont pluri-cellulaires (1) et finissent par fructifier après la production d'un nombre plus ou moins grand de cellules végétatives ou frustules. - Nous considérons les Diatomées comme des algues pluri-cellulaires filamen-

(1) C'est à dire composées de plusieurs frustules unicellulaires.

J. P. 
teuses, ou cryplofilamenteuses, selon que le thalle en est persistant pendant la vie de la plante, ou qu'il se sépare en ses éléments constitulifs au fur et à mesure que ceux-ci se développent. Le sporange, dans les deux cas, est la fin d'une série.

Déduplication des Diatomées. - Le mode de division des cellules des Diatomées, ou comme on l'exprime généralement, la déduplication des frustules, se fait d'après un plan spécial commun à toutes les Diatomées filamenteuses ainsi qu'à celles à frustules isolés, et qui diffẻre essentiellement de ce que l'on observe chez d'autres organismes (1).

Nous allons tàcher d'expliquer d'une façon aussi claire que possible le phénomẻne de la déduplication ou de la croissance des Diatomées.

Une Diatomée réduite à sa plus simple expression, c'est-à-dire la premiẻre cellule d'une plante nouvelle, représente à l'imagiriation une petite boîte dont l'enveloppe est formée de cellulose dont la substance est tellement imprégnée de silice que cette dernière prédomine généralement au point de rendre cette pellicule cassante, incombustible et inattaquable

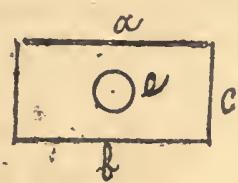

1

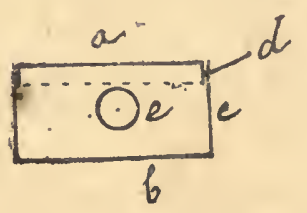

2

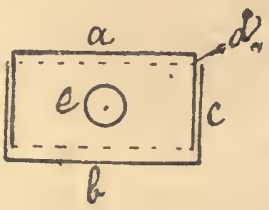

3

Fig. 1. - Frustule théorique de Diatomée. - $a$, valve supérieure; $b$, valve inférieure ; $c$, connectif unique ; $e$, noyau. - Fig. 2. - Commencement de la déduplication; développement du second connectif $d$. (Mêmes lettres que dans la fig. précédente). - Fig. 3. - Suite de la déduplication et de la formation du connectif nouveau (Mêmes lettres).

par les acides. Un examen attentil sous l'objectif d'un bon microscope avec un fort grossissement, nous démontre que notre petite boîte est constituée en réalité par trois parties distinctes : un couvercle qui s'appelle la valve supérieure, un fond de la boîte qui s'appelle la valve inférieure e un anneau qui sépare les deux valves et qu'on appelle le connectif. (Fig. 1). Ce connectif adhère assez solidement à la valve inférieure, mais ne tient que très légèrement à la valve supérieure.

Dans l'intérieur de la boîte qui constitue le frustule de la Diatomée, on aperçoit, à travers la paroi transparente, un noyau central ou nucleus et souvent un nucléole, puis une masse de protoplasme ou matière vivante; puis enfin, des matières colorantes, des corps gras et quelques corpuscules

(1) Braun, dans son ouvrage a Algarum Unicellularium, " avait déjà, dès 1855, pressenti la pluri-cellularité des Diatomées, contrairement aux vues de Nægeli. 
indéterminés. Ce contenu prouve à toute évidence que le frustule d'une Diatomée n'est quiune cellule constituée sur le même plan que toutes celles des corps or'ganisés.

En suivant avec patience l'évolution d'un frustule, on observe les phénomènes suivants :

$1^{\circ}$ Le couvercle de la boîle siliceuse ou valve supérieure sécrète peu a peu un anneau de silice tout autour de son bord interne (Fig. 2, 3. d).

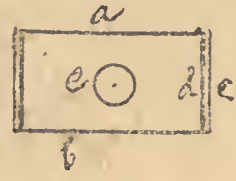

4

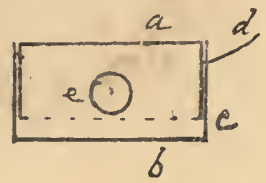

5

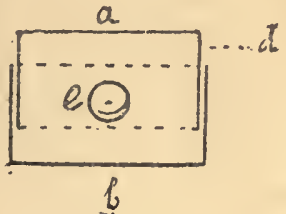

6

Fig. 4. - Suite. - Le connectif noureau $d$ est complet. (Mêmes lettres). Fig. 5. Commencement de l'écartement entre les deux valves. (Mêmes lettres). - Figg. 6. Ecartement des deux valves. (Mêmes lettres).

Cet anneau grandit peu à peu depuis le haut vers le bas de la boîte en s'appliquant étroitement à la face interne de l'anneau primitif. En fin de compte, cet anneau ou connectif nouveau atteint le fond de la boite ou la surface interne de la valve inférieure. (Fig. 4. d). Ici son développement s'arrête et nous avons sous les yeux un frustule constitué de deux valves et de deux connectifs, ces derniers superposés.

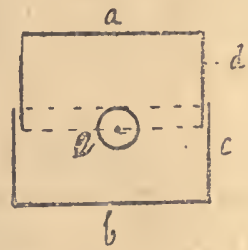

7

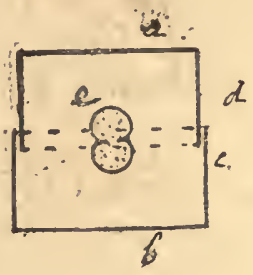

S

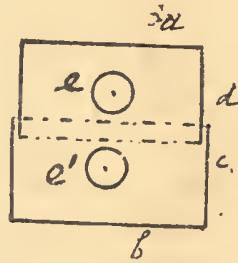

9

Fig. 7. L'écartement des deux valves atteint son maximum. - Fig. 8. Commencement de la division du noyau, (Mêmes lettres). - Fig. 9. Division complète du noyau. (Mêmeslettres).

2० Aussitôt que le connectif nouvellement formé a alteint la valve inférieure, les matiẻres vivantes contenues à l'intérieur du frustule augmentent rapidement en volume et par leur pression forcent l'écartement des deux valres, ce qui s'effeclue par le glissement graduel du connectif nouveau dans le connectif primitil comme dans une gaine ou tirant de lunette d'approche. (Fig. :ै, 6.) Cet écartement se continue graduellement jusqu'au moment où les bords libres des deux connectifs sont très près de se rapprocher. (Fig. 7.) Un peu plus et le couvercle de la boîte ou valve supérieure avec son connectif se sépareraient entièrement de la valve inférieure el de son connectif, mettant à nu le contenu du 
frustule. Mais avant ce moment critique, l'écartement des deux valves a cessé et le frustule a atteint sa largeur maxima.

Après que le glissement des connectils a atteint son maximum d'expansion, le nucléus intérieur commence à s'étrangler par le milieu (Fig. 8) et la matière de la cellule protoplasmique avec ses contenus commence à se partager en deux portions égales.

Quelques instants plus tard, le nucléus s'est divisé en deux (Fig. 9) et chaque moitié constitue un nucléus nouveau qui va occuper l'une des moitiés du protoplasme qui, maintenant aussi, est distinctement séparé en deux masses par une ligne de démarcation visible ả l'œil.

$4^{\circ}$ Chacune des deux masses protoplasmiques commence alors à sécréter, à sa surface nue, une mince pellicule siliceuse (Fig. 10) qui, par des accessions graduelles de silice, s'épaissit et reproduit exactement la valve qui lui est opposée. Dis ce moment la déduplication s'est effectuée et le

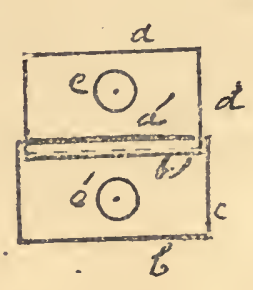

10

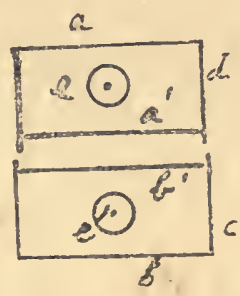

11

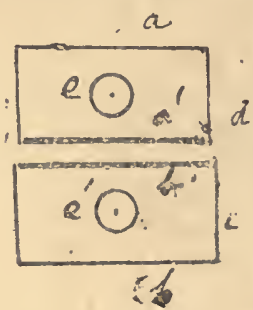

12

Fig. 10. Formation des nouvelles valves; $a$, nouvelle valve forméc dans le connectif de la valve supérieure; $b^{\prime}$, nouvelle valve formée dans le connectif de la valve inféricure. (Mêmes lettres). - Fig. 11. Séparation des deux fructules. (Mêmes lettres). - Fig. 12. Résorption des rebords du connectif; déduplication achevée. (Mrêmes lettres).

frustule|primitif se trouve transformé en un corps bi-cellulaire. Le frustule maintenant est réellement formé de quatre valves dont deux internes et deux externes, le tout retenu par deux connectifs apparienant à chacune des valves primitives. Le frustule mère a été modifié de maniẻre à ce que chacune de ses filles est constituée par l'une des valves de la mère et une valve nouvelle formée à l'intérieur de l'un des connectifs de ces valves mêres.

$\check{\jmath}^{\circ}$ Un léger glissement final des connectifs sépare bientôt les deux nouveaux frustules l'un de l'autre (Fig. 11) et les valves jeunes vont alors se placer au bord libre même des connectils pour reproduire exactement la cellule primitive qui a commencé la série; puis, les deux frustules se séparent (Fig. 12). Chacune des jeunes valves dont nous avons suivi le développement pas à pas, va à son tour passer par toutes les mêmes phases qui l'ont formée et celte succession de déduplications se continuera indéfiniment jusqu'à ce que la fructification de l'algue y mette fin.

Il est évident que, dans le cas des Diatomées filamenteuses, le glissement 
des connectifs n'a jamais lieu d'une manière complete et que les divers frustules restent attachés l'un à l'autre par l'extrémité des connectifs pendant toute la durée de l'existence de l'algue. Le mode de déduplication des cellules est d'ailleur's parfaitement le mème pour' les formes à union permanente que pour celles dont les éléments se dissocient à mesure de leur création.

La plupart des frustules des Diatomées, pendant au moins une certaine période de leur croissance, sont couverts ou protégés à l'extérieur par une matière amorphe sécrétée par l'organisme lui-même. C'est le coléoderme appelé improprement le thalle par M. le Dr Lanzi. Cette sécrétion est anhiste et peut prendre un développement très considérable ou bien, dans cerlains cas, se réduire á une simple pellicule adhérente aux valves. Elle peut aussi, comme cela'se voit chez les Schizonema, les Colletonema, les Encyonema, former des tubes qui hébergent les frustules, ou bien des tiges plus ou moins rigides qui les supportent comme dans les Gomphonema, les Achnanthes et beaucoup d'autres genres. C'est d'ailleurs une matière morte et inerte, qui ne doit pas êtrè confondue avec la matière vivante intérieure du frustule qui ne communique pas plus directement avec l'extérieur que cela n'a lieu chez d'autres cellules organiques vivantes.

Nous ne pouvons nous étendre longuement sur le contenu des cellules ou frustules des Diatomées, ce sujet ne présente d'ailleurs guère de faits particuliers. Le noyau et le nucléole sont identiques à ceux d'autres plantes.

La matière protoplasmique interne tapisse tout l'intérieur du frustule et se groupe en masses centrales et souvent terminales. Elle est le porteur des plaques ou des grains colorés ou chromatophores et présente de nombreuses vacuoles remplies d'un liquide incolore dans lequel flottent quelques globules huileux - Avec une grande attention, on découvre des filets protoplasmiques qui s'étendent entre la couche périphérique et celle qui entoure le noyau. Dans de fort bonnes conditions d'outillage et d'éclairage on peut distinguer même une circulation ou cyclose dans ces filets qui sont quelque peu finement granuleux.

Mouvernents des Diatomées. - Beaucoup de frustules de Dialomées libres sont doués d'un mouvement propre dont on n'a pas encore bien compris la cause, mais qui paraît mécanique ou due à des causes physiques. Nous sommes tentés de l'attribuer principalement à un phénomėne de capillarité, qui se manifesterait entre les conneclifs, ou le long des bords des valves. Les opinions à l'égard de ces mouvements des Diatomées sont extrêmement variées, mais aucune ne répond aux besoins de la cause.

Structure microscopique des Diatomées. - La question de la structure intime microscopique des valves des Diatomées constilue depuis longtemps le champ de bataille des micrographes et sa solution est l'un des 
problèmes les plus difficiles à élucider que l'on puisse imaginer. L'on ne peut l'aborder qu'armé des meilleurs et des plus forts objectils de notre époque et encore faut-il savoir s'en servir avec tous les raffinements modernes. Quand l'écaille siliceuse d'une Diatomée a été vue et mème dessinée, au microscope, il reste à interpréter ce qu'on a vu, pour séparer ce qui est réel de ce qui n'est dû qu'à des phénomènes compliqués d'interférence, de diffraction, de réfraction et autres causes d'illusions optiques qu'il est malaisé de débrouiller. Les fins dessins qu'on aperçoit, sous forme de stries, de côtes, de points, de réticulations se résolvent sous nos plus puissants instruments en détails si petits, si infiniment petits même, qu'on ne sait plus les apprécier avec certitude. C'est ce qui a causé tant de controverses sur leur nature qu'il m'est de toute impossibilité de passer en revue dans ces quelques pages sommaires.

Je ne puis que résumer mon opinion, toute personnelle, à l'égard de la structure intime des valves des Diatomées et qui me fait croire que le dépôt de la silice se fait à l'intérieur des jeunes valves, au moment de la déduplication, par l'intermédiaire des courants protoplasmiques qui lui servent de porteurs. Ces courants ou filets cyclotiques varient de position, allant tantôt dans un sens, tantôt dans un autre. Les dépôts se font aussi en dessins sous forme d'anastomoses, dont les orifices se remplissent de plus en plus de silice tout en laissant toujours un lumen. Ce filet siliceux anastomosé s'adapte à une couche de cellulose homogène et continue qui constitue la face externe de la valve. - A l'intérieur, lors de la maturité, le fond des petites cavités se recouvre également d'une membrane siliceuse, quelquefois même feuilletée.

Je pense qu'une Diatomée qui présente à là vue des points, des hexagones, ou d'autres dessins délicats, montre en section des cavités nombreuses fort petites, recouvertes au-dessus et au-dessous par une membrane homogène continue. L'une ou l'autre ou chacune de ces cloisons peut-porter des dessins encore plus petits qui lui sont propres. Chez les Diatomées fossiles, comme dans celles qui ont été soumises à l'action du feu ou des acides, les fines membranes externes ont disparu et dans ces cas la membrane du frustule est bien réellement perforée de trous, et forme tamis, comme cela a souvent été décrit. Nous ne pensons pas à la possibilité d'un contact direct du protoplasme du frustule avec les milieux ambiants. La cellule prend sa nourriture par intussusception ou par capillarité ; peut-être aussi par endosmose à travers ses membranes.

Elle n'absorbe que des liquides et jamais il ne pénètre à l'intérieur du frustule des particules solides quelle que soit leur exiguité.

Reproduction des Diatomées. - Comme nous l'avons dit précédemment, le thalle après avoir donné naissance à un nombre plus ou moins considérable de cellules végétatives ou de. frustules, finit par développer un ou plusieurs fruits ou sporanges. Ces sporanges sont toujours formés au dépensdu contenu d'un $\cdot$ ou de plusieurs frustules qui ne different 
généralement des autres que par une taille quelque peu moindre. Ces frustules, après s'être entourés de mucus, séparent leurs valves et s'ouvrent. Leur contenu mou protoplasmique, devenu libre, s'arrondit alors et s'enkyste pour former ce que l'on a nommé des auxospores. Après un repos plus ou moins long, ces auxospores produisent à leur tour, soit des frustules ordinaires, analogues à ceux qui commencent une série ou plante nouvelle, mais de forme plus grande, soit des spores, en nombre plus ciu moins grand, qui sont propres à reproduire, chacun, une plante nouvelle par leur développement ultérieur. Malheureusement, malgré un nombre très considérable de faits constalés, ce que nous savons de tout ce qui concerne la reproduction des Diatomées resle peu compris et mal élucidé. C'est un champ ouvert à l'observateur patient qui voudra écrire l'histoire complète d'une seule des dix mille espèces connues de Diatomées, travail qui reste encore à faire, à la honte réelle des micrographes et des naturalistes.

Une préface comme celle-ci ne peut constituer qu'un résumé bien court de ce qui va suivre dans le corps de l'ouvrage, je me vois donc forcé d'en arriver à une conclusion, en priant le lecteur de bien vouloir m'excuser d'ètre si laconique. Je le fais a vec la certitude qu'on n'aura pas à se plaindre du travail de notre savant ami M. le $\mathrm{D}^{\mathrm{r}}$ Pelletan dont l'érudition et la clarté de vues sont devenues proverbiales.

Je ne puis, en terminant, que recommander instamment l'étude si altrayante de ces véritables joyaux de la nature, les Diatomées. Rien n'est plus facile que de s'en procurer, car il y en a partout, sur la terre humide, dans les mousses des arbres el des vieux murs, sur les rochers arrosés par des sources, dans les ruisseaux, les rivières, les étangs, les fossés et les lacs d'eau douce, jusqu'au sommet des hautes montagnes el sous toutes les latiludes. Dans les eaux saumâtres, elles abondent, non seulement près de la mer, mais même dans les salines artificielles de l'intérieur des continents.

Enfin, la mer en fournit un nombre extrêmement grand, de formes rares et belles, dont les unes sont essentiellement pélagiques et passent leur existence flottant à la surface de l'océan, d'autres ne se récoltent que dans les abîmes les plus profonds qu'on ait atteints avec la drague, tandis que le plus grand nombre ne se trouvent que dans la zone littorale.

Un nombre très grand d'animaix se nourrissent presqu'exclusivement de Diatomées et les carapaces siliceuses et indigestes de ces dernières remplissent, en ce cas, leurs cavités digestives qui constituent dès lors de vérilables magasins ou des boîtes ả surprise pour le naturaliste. - Les Dialomées ont vécu, dans les temps géologiques, aussi bien dans les eaux douces que dans les mers anciennes, comme le témoigneut d'immenses dépôts qu'on rencontre dans les cinq parties du monde, presqu'exclusi- 
vement formés par leurs frustules impérissables. Beaucoup de ces formes des âges passés sont de races éteintes aujourd'hui.

Il y a près de quarante ans queje m'occupe pendant mes loisirs, hélas ! trop peu nombrẹux, de l'étude des Diatomées. J'y ai trouvé, pendant cette longue partie de mon existence, un délassement bienfaisant, une récréation saine et de bon aloi, un plaisir continu, qui m'unt maintes fois fait oublier momentanément les petites et les grandes misères d'une vie fort accidentée. Je souhaite ì tous ceux qui suivront mes conseils de trouver dans l'étude de ces admirables petils organismes autant de satisfaction que j'en ai trouvé moi-même.

Un vaste champ reste ouvert pour compléter l'histoire des Diatomées, il y a donc gloire et profit à retirer de leur étude pour celui qui voudra sérieusement l'entreprendre.

J. Deby.

Hampstead (London), 26 sept. 1887. 


\section{HISTORIQUE}

Le premier naturaliste qui, d'après Ehrenberg, observa une espèce appartenant à la famille des Diatomées serait Leeuwenhoek qui, en 1703, aurait vu sur des Algues vertes un organisme que le même Ehrenberg dit être le Synedra ulna. Cette découverte serait consignée dans un mémoire a concernant les Algues vertes qui poussent dans l'eau et quelques animalcules qui se trouvent près d'elles (1), "mémoire inséré dans les Philosophical Transactions de la Société Royale de Londres pour l'année 1703.

C'est là une erreur du célèbre micrographe allemand. Nous nous sommes reporté au travail original de Leeuwenhoek cité par lui : c'est une lettre, datée de Delft, en Hollande, le 25 Décembre 1702, faisant partie du n 283 du Recueil des Transactions. Celte lettre est uniquement consacrée á la description de quelques Infusoires Vorticelliens et de la petite plante que tout le monde connait sous le nom de lentille d'eau (Lemna major ou minor) et qu'on appelait alors Lens palustris. La planchequi accompagne cette lettre représente d'une manière très reconnaissable et la plante et les animalcules. Quant à la figure 8, spécialement désignée par Ehrenberg comme relative à une Diatomée qui serait le Synedra ulna, elle représente très neltement une radicelle grossie de Lemna, sur laquelle sont fixés des Vorticelles et des Vaginicoles. Peut-être la radicelle est-elle un peu trop droite et raide, le dessin de cellules peut-être un peu trop régulier, mais il est impossible de se méprendre sur ce qu'a voulu figurer Leeuwenlıok.

Mais le volume 1702-1703 des Transactions contient plusieurs lettres ou parties de lettres de "M. Anthony van Leeuwenhoek. " Dans une de celles-ci, datée du 5 février 1703, (2), cẹt auteur raconte qu'en juillet $\mathbf{1 7 0 2}$, en laissant au repos de l'eau prise dans un canal, à Delft, il y a vu des corps microscopiquies réguliers et transparents, formant comme des arbo-

(1) " Concerning green Weeds growing in water and some animalcula about them, by M. Lecuwenhock, Ph. Tr. 1703, n² 283.

(2) Part of letter from M. Anthony van Leeuwenhoek containing his observations on some animalcula in water, etc, - Ph. Trans. 1703, T. XXIII p.1431. 
risations. Il les compare à des cristaux et indique comment il a cherché à les imiter en laissant évaporer des solutions faibles d'un sel d'argent, produisant en petit le phénomène connu sous le nom d'arbre de Diane.

Est-ce un dépố de Diatomées que I_eeuwenhoek a observé? - Cela est fort probable, et quelques-unes des figures qu'il donne (notamment celle qui porte le $n^{\circ} 1$ ) sembleraient le prouver. Dans ce cas, il serait le premier observateur donl l'attention a été fixée sur une Diatomée.

$\mathrm{Au}$ cas contraire, la découverte de ces pelites Algues doit néanmoins être placée dans l'année 1703 - car, dans le no 286 des mêmes Philosophical Transactions (1) se trouvent deux parties de lettres adressées par un "gentleman in the country " dont le nom n'est pas donné, lettres dont Ehrenberg n'a pas eu connaissance non plus que de la précédente.

Dans la seconde de ces deux lettres (5 juiliet 1703) il est dit :

- Dans mes observations sur ces plantes, (racines de lentilles d'eau), j'ai souvent vu, adhérentes à ces tiges ou quelquefois séparées dans l'eau, beaucoup de petiles branches composées de pièces rectangulaires oblongues ou exactement carrées, réunies entr'elles comme dans le fig. VII que j'ai dessinée aussi exactement que je l'ai pu d'après nature (2). Il y a quelquefois vingt de ces pièces, ou davantage, dans une branche qui généralement adhẻre par une de ses exlrémités aux tiges de la plante, et il est remarquable, je pense, que ces parallélogrammes rectangulaires sont toujours de la même taille, le long côté n'excédant pas le tiers de l'épaisseur d'un cheveu, les carrés étant visiblement composés de deux parallélogrammes réunis par le long côté. Ils paraissent très minces, et la structure de chacun semble à peu près la même. A un très fort grossissement, ils apparaissent comme dans la Fig. VIII (3). J'ai d'abord pris ces branches pour des sels, mais trouvant qu'elles avaient toujours la même taille et qu'elles ne prenaient pas un accroissement sensible de volume tout le temps qu'elles restaient dans l'eau, qu'après avoir été un jour ou deux à sec sur une lame de verre elles ne s'altéraient pas dans leur forme, et par l'addition d'une nouvelle eau (chaude ou froide) présentaient toujour's la mème apparence et la mème cohésion, et que leur adhérence (quoiqu'elles ne se touchent que par les points angulaires) était si forte et si rigide que toutes se mouvaient ensemble et gardaient la même position les unes par rapport aux autres bien qu'elles fussent agitées par l'eau; ces consi-

(1) Letters from a Gentleman in the country relating to $M$. Leeuwenhock's letter in Transactions $n^{\circ} 283,-$ communiquées par M. C. (pas de nom). Ph. Trans. T. XXIII, 1703, nº 286, p.1499. - (La première est datée du $1^{\text {er }}$ juin, la seconde du 5 juillet 1703$)$.

(2) Ce n'est pas celte figure que cite Ehrenberg comme étant de Leeuwenhoek, mais une figure 8, Planche I, qui représente, comme nous l'avons dit ci-dessus, une radicelle de Lemna.

(3) Cetle figure représente trois frustules un peu plus grossis. 
dérations, dis-je, me persuadèrent qu'il s'agissait plutôt de plantes que de sels, mais comme elle sont si petites qư'aucun jugement ne peut en être porté par l'œil, je ne veux rien déterminer positivement. '

Cet observateur anonyme a donc été tenté de prendre aussi cette série de frustules pour des cristaux, comme il paraît d'autant plus probable que l'a fait Leeuwenhoek dans son observation de juillet 1702. Néanmoins il revient à l'idée que c'est une plante et la description qu'il en donne, comme le fait remarquer M. F. Kitton (1) est suffisante pour faire reconnaître le Tabellaria flocculosa des diatomistes modernes et non le Synedra ulna comme l'a dit Ehrenberg. La figure d'ailleurs, fort bien dessinée, ne laisse pas de doutes.

Puis, vint Louis Joblot qui, en 1714-1716, découvrit une espèce nommée par lui Vibrio Bacillus, laquelle semble être le même Tabellaria (Observations faites avec le microscope).

En 1745̈, William Arderon découvrit un animal-avoine " (oat-like animal) qu'il avait obser'vé sur l' " animal-cheveu " (hair-like animal) et qui a été longuement décrit et mêne figuré dans l'Employment for the Microscope, de Baker, paru en 17533.

Dans ce temps là, on avait assez l'habitude de désigner ces êtres microscopiques, jusqu'alors inconnus, en les comparant à des objets connus. C'est ainsi qu'on avait l'animal-cheveu, qui est une Oscillaire, l'animaltrèlle, qui est un Rotateur, un Chxtonotus, l'animal-fève, qui est un Infusoire cilié ; quant à l'animal-avoine, c'était sans doute une Diatomée appartenant au groupe des Navicules, peut-être le Navicula sphærophora ou le $N$. amphisbana.

C'est encore un Navicula, peut-être le $N$. fulva, que Schrank décrivit plus tard (1776) sous le nom de Chaos infusorum.

Le célèbre naturaliste 0tto Frédéric Müller observa, en 1773, ả Pỵ-mont, le Gomphonema truncatum dont il fit un Vorticella pyraria; en 1779, l'Achnanthes brevipes, el en 17S2 son Vibrio paxillifer qui n'est autre que la singulière Diatomée appelée depuis Gmelin jusqu'ì présent Bacillariaparodoxa. A ces espèces, il ajoula, en 1783, les Conferva pectinalis, qui est un Fragilaria actuel, et Conferva armillaris, qui est un Melosira. II décrivit encore, dans son grand ouvrage posthume publié en $\mathbf{1 7 8 6}$ (2), un Vibrio bipunctatus, qui est sans doute le Synedra ulna, et un Vibrio tripunctatus qui est le Navicula gracilis, espèces qu'Hermann avait observées en $\mathbf{1 7 8 4}$ et désignées sous le nom d'Enchelys.

Schrank, en 1797, décrivit encore deux Navicules sous' les noms de Vibrio turrifer et $V$. fuscus, et un Cocconema sous celui de Kolpoda luna.

(1) F. Kitton. - Early history of Diatomacex, dans lc Science Gossip, 1880.Traduit dans le Journal de Micrographie, 1880.

(2) 0. F. Müller, Animalcula Infusoria fluviatilia et marina, etc. Hauniæ, . 1786 , in- $4^{\circ}, 50$ planches. 
On le voit, le nombre des Diatomées connues à l'époque ou parut le célèbre ouvrage de 0 . F. Müller, était encore bien pelit. Néanmoins, les difficultés étaient déjà grandes pour les naturalistes qui les avaient observées et qui cherchaient à les classer, les uns dans le règne animal, les autres dans le régne végétal, d'autres encore à les distribuer dans les deux règnes. Et les difficultés étaient d'autant plus grandes que parmi les formes microscopiques, qu'ils découvraient, il y avait non-seulement des Diatomées véritables, mais deș Infusoires, des Acinètes, qui présentent des caractères d'animalité bien tranchés, et des Algues qui on!, au contraire, une physionomie nettement végétale. Cet embarras des classificateurs se révẻle, d'ailleurs, par l'attribution qu'ils font des espèces nouvellement décrites les unes au genre Vibrio, les autres au genre Conferva.

C'est ainsi que Gmelin, créateur dugenre Bacillaria, qu'il institua, en 1788, pour le fameux Vibrio paxillifer de 0. F. Müller dont il fil le Bacillaria paradoxa, rangea ses BaclLLaRıÉEs dans le règne animal, tandis que Vahl, dans sa Flora Danica, le rédacteur de l'English Botany, puis Roth, qui donne même une bonne figure due à Mertens d'une Diatomée dont il fait un Conferva flocculosa, les replacèrent parmi les plantes. D'autre part, vers la même époque, Schrank en fait des Vibrio et des Kolpoda, c'est-à-dire des animaux, avec Kammacher, qui, dans la Micrographia d'Adams (1798) range un Navicula dans le règne animal (1).

A partir de cette époque, les travaux sur ces organismes devinrent de plus en plus nombreux, mais les idées étaient encore bien loin d'être fixées sur leur position systématique, car Girod Chantrans en faisait des Algues qui produisaient des animaux, et Ingenhousz, ne pouvant en faire ni des bétes ni des plantes, ne trouva rien de mieux que de les classer dans le règne minéral (1802).

Cependant, c'est dans les Algues que la majorité des observateurs de la fin du XVIII e siècle classaient les organismes qui nous occupent, et notamment parmi les Conferves, en raison de la forme filamenteuse qu'alfectent un grand nombre d'espèces. Et, en réalité, c'est surtout les algologues qui s'adonnèrent à cette étude. C'est ce que firent Roth, Mertens, Trentpohl, Weber, Hornemann, Mohr, Dillwyn, Thore, Draparnaud. Agardh et, enfin, le célèbre botaniste de Candolle qui, en 1805, créa le genre Diatoma (du grec ò $\_\tau \kappa \mu \nu \omega$, diviser), auquel il donne pour type le Conferva flocculosa de Roth et qu'il plaça parmi les Algues.

Dillwyn (2) fit entrer aussi, sous le nom de Conferves, un certain nombre de Diatomées filamenteuses dans ses British Conjervæ (1809) et en

(1) Roth. - Catalogus Botanicus, Fasc. I, Tab. IV, fig. 4 êt Tab. V, fig. 6 (1797).

(2) Dillwyn. - British Confervx, in-4으, 1809. 
donna des figures qui permettent de les reconnaittre aujourd'hui (Fig. 13).

L'exemple de de Candolle fut suivi par le grand algologue Agardh qui adopta le genre Diatoma, l'étendit et le fit entrer dans sa Synopsis Algarum (1817).

C'est en se plaçant à un tout autre point de vue que Nitzsch se livra à des recherches approfondies, et de tous points excellentes, sur la constitution de ces petites plantes dont il donna réellement les premières figures exactes dans son livre publié en 1817 (1). Il reconnut leurs formes, ob-

1.

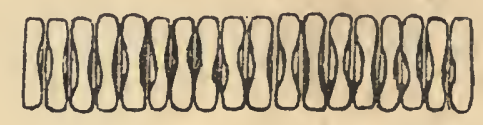

2.

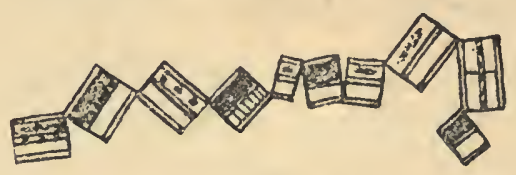

3.
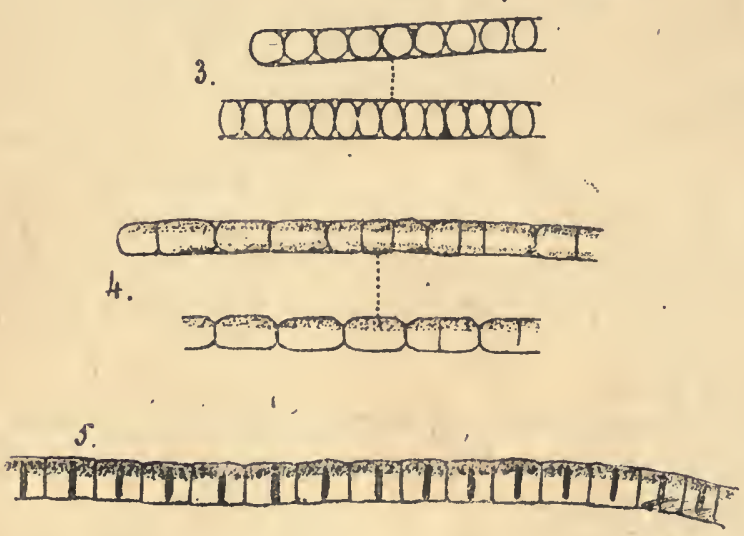

Fig. 13. - Diatomées figurées par Dillwyn dans les British Confervæ (1809).

1. - Conferva pectinalis, Dill. (Himantidium pectinale des auteurs modernes). - 2. Conferva flocculosa, Dill., Roth. (Tabellaria flocculosa, aut. mod.) - 3. Conferva nummuloides, Dill. (?) - 4. Conf. lineata (Melosira lineata, aut. mod. - 5. Conf. fasciata, Dill. (Melosira varians, aut. mod.).

Ces figures sont soigneusement copiées sur celles de Dillwin qui représentent ces Algues sous le " sixième pouvoir » de son microscope, c'est-ådire, â ce qu'il semble, 200 diamètres. Les ombres représentent l'endochrome coloré en vert dans les figures originales (2).

(1) D. Ch. Ludw. Nitzsch-Beiträge zur Infusorienkunde oder Naturbeschreibung der Zercarien und Bacillarien, avec 6 pl. sur cuivre, en couleurs. Halle, 1817, in- $8^{\circ}$.

(2) F. Kitton. Notes sur les genres et les espèces des Diatomées compris dans les "British confervae " de Dillwynn. (Journal de Micrographie, T. Vill, 1883, p. 526 et Science-Gossip, 1883.) 
serva la multiplication des "bâtonnets " par division longitudinale, ce qui lui permit d'expliquer facilement la formation des filaments et des chaînes en zig-zag, et signala la persistance de l'enveloppe après la mort de l'organisme. Mais il en considéra le plus grand nombre comme de véritables animaux, en raison de leurs mouvements, tandis que d'autres étaient récllement des végélaux. Et cependant, parmi les espèces qu'il regardait comme des plantes, plusieurs sont douées de mouvement.

Puis, Lyngbye publia, en 1819, son Tentamen Hydrophytologix Danic: $x$, livre très important pour l'époque et dans lequel il décrivit beaucoup d'espèces Bacillariées nouvelles, remania et compléta les genres Diatoma, Fragilaria et Echinella. Ce dernier a disparu des classifications el renfermait des organismes qui ont été reconnus plus tard pour des œufs d'insectes.

Pour Lyngbye, toutes ces espèces appartenaient au règne végétal. Ce ful aussi l'opinion de Link et de Bonnemaison. Le premier décrivit en $\mathbf{1 8 2 0}$ les deux nouveaux genres Hydrolinum et Lysigonium (Melosira et Schizonema), et le second, les genres Vaginaria et Spermogonia (Schizonemu).

C'est alor's que Bory de Saint-Vincent établit, dans le Dictionnaire. classique d'Histoire Naturelle, sa famille des Antunodiées qu'il place comme intermédiaire entre les animaux et les végétaux, et dans laquelle il fait entrer les Achnanthes et plusietirs aulres genres et espèces de Diatomées. Puis, il créa le genre. Navicula qu'il raltacha à la famille des Bacillariḱes, laquelle, réunie aux Infusoires, forma son groupe des PSYCHODÉES.

"Mais, dit Külzing, tandis que Bory de Saint-Vincent fondait surtout ses genres sur les recherches des autres observateurs et que les quelques investigations qui lui sont personnelles portent trop l'empreinte d'une étude superficielle, les travaux de G. A. Agardh sur les mémes groupes sont plus approfondis. Dans son Systema Algarum (1824), cet auteur mentionne les Bacillariées comme un ordre spécial des Algues, sous le nom de Diat o MéEs, et les classe mieux et plus complètement que ses prédécesseurs, dans les genres: Achnanthes, Frustulia, Meridion, Diatoma, Melosira, Schizonema, Gomphonema et Desmidium ». Ce dernier genre a, dẹpuis, été séparé des Dialomées et a formé le type de la famille des Desmidiées.

Dans les: années suivantes, le même naturaliste agrandit encore le cadre de la famille des Diatomées qui, en 1830, ne comptait pas moins de 21 genres, la plupart conservés anjourd'hui, et contenant environ $\mathbf{1 1 6}$ espèces. Pendant ce temps, d'ailleurs, plusieurs botanistes éminents avaient enrichi cette famille de nouveaux genres et de nouvelles espèces : Gaillon, Link, Leiblein ef surtout Greville (1827) et le célèbre botaniste Turpin, l'un des précurseurs de la théorie cellulaire et le créateur du genre Surirella, en 1828; puis Gray, en 1832. 
"Ainsi, dit encore Kützing, jusqu'en 1832, les travaux systématiques s'arrêtèrent à ces organismes microscopiques; beaucoup des auteurs mentionnés les considéraient, en partie comme animaux (les formes mobiles), en partie comme végétaux (les formes fixes). Agardh, Lyngbye et Leiblein seulement se déclarèrent plus décidément pour le caractère végétal; mais, excepté Schrank, aucun ne soutint formellement leur nature animale. De leur constitution intime et de leurs fonctions vilales, rien, en dehors des observations complètes données par Nitzsch et de quelques indications superficielles de Gaillon, rien n'était connu qui avancât la question relative à leur nature „- En cette même année, 1832, parul la seconde Contribution à la connaissance des organismes microscopiques de C. G. Ehrenberg. Dans cet ouvrage, les Diatomées furent décidément considérées comme des formes animales.

Cette répartition des Diatomées parmi les Infusoires a été soutenue par Elırenberg, dans les trèsnombreuses publications qu'il fit paraître pendant les années suivantes, et définitivement consacrée dans son grand ouvrage : Les Infusoires considérés comme des organismes parfaits (1). Il les incorpore à sa famille des Polygastriques, regardant la matière colorée qu'elles contiennent comme des ovaires, et les globules plus clairs qui y sont répandus, par exemple dans les Navicula, comme les diverses parlies d'un estomac à plusieurs lobes.

Les observations de l'illustre micrographe eurent un immense relentissement et exercèrent une influence considérable sur la marche de la science des infiniments petits. Elles furent faites, d'ailleurs, avec un soin inconnu jusque lả dans ces sortes de recherches, accompagnées de planches superbes et d'une admirable exactitude dans le dessin général. Ehrenberg était, du reste, supérieurement placé pour donner à ses observations une étendue et une rigueur auxquelles la plupart de ses prédécesseurs n'avaient pu atteindre. Disposant des instruments les plus parfaits que construisaient les opticiens de son temps, profitant de ses relations dans les deux contiıents, recevant des matériaux de tous les points de la terre, il put rassembler des documents en nombre considérable, étudier les formes fossiles et même retrouver à l'état vivant des espèces considérées jusqu'alors comme éteintes. C'est ainsi que pendant de longues années, et presque jusqu’à nos jours (1875)), il publia de nombreux ouvrages, notamment sa Mikrogeologie (183\%) et des mémoires plus nombreux encore insérés dans les Comptes rendus de l'Académie des Sciences de Berlin.

Dès l'époque où parut son grand ouvrage sur les. Infusoires, il y incorporait $\mathbf{2 4}$ genres comprenant 144 espèces de Diatomées, nombre qu'il augmenta considérablement dans les années suivantes.

Mais, pendant cette longue période, les observateurs s'étaient multipliés

(1) C. G. Ehrenberg: Die Infusionsthierchen als vollkommene Organismen, 2 vol. in-fol. Leipzig, 1838. 
de tous côtés et beaucoup d'entr'eux avaient porté leur attention sur cette famille des Diatomées. Ce fut d'abord A. de Brébisson, dont nous retrouverons à chaque instant le nom dans l'histoire de ces organismes; et, contrairement aux idées d'Ehrenberg, il les rangea dans la classe des Algues où, depuis lors, tous les naturalistes se sont accordés à les maintenir.-Puis, Bailey, Gréville, Külzing. - Ce dernier auteur avait, dẻs 1833, publié des iravaux importants sur les Diatomées ou Bacillariacées à enveloppe siliceuse, et établi une classification (1), que Brébisson adopta en grande partie.

A partir de ce moment, à mesure que ces formes si particulières de la vie végétale sont mieux connues, on voit de tous côtés surgir des chercheurs qui viennent enrichir la science de notions plus élendues etplus complètes. En même temps, cette étude se spécialise, pour ainsi dire, de plus en plus, et devient l'apanage de nombreux savants qui font des Diatomées l'objet à peu près exclusif de leurs recherches. Tels furent : Ralfs, Harting, Pritchard, Brigthwell, William Smith, Shadbolt, Rabenhorst, Gregory, Max Schultze, Roper, Walker Arnolt, Wallich, O'Meara, Donkin, A. M. Edwards, Janisch, P. T. Clève, Grünow, Hamilton Lawrence Smith, Eulenstein, Guinard, Paul Petit, Van Heurck, Castracane, Kitton, Flögel, Pfitzer, Ad. Schmidt, Matteo Lanzi, Cox, Julien Deby, Leuduger-Fortmorel, Otto Müller et un grand nombre de diatomistes dont nous ne pouvons citer ici les noms, qui ont fait de l'étude de cés admirables petites plantes une branche spéciale de l'histoire naturelle et de la micrographie.

Les détails si intéressants de leur organisation, les phénomènes biologiques si curieux dont elles sont le théâtre, l'élégance et la finesse des sculptures dont leur enveloppe est ornée, la difficulté même qu'offre souvent l'étude de ces mêmes sculptures, la profusion avec laquelle ces espèces sont répandues dans la nature, suffisent bien pour expliquer l'irrésistible attrait que les Diatomées ont exercé, depuis qu'elles sont connues, sur tant d'observateurs qui se sont passionnés pour ces délicates recherches.

Ajoutons que jusqu'à ces dernières années où l'étude des Bactériens est venue prendre une place importante dans les travaux des chercheurs, aucune branche de la science n'a été plus profitable à la micrographie; car, outre qu'elle a forcé les opticiens' ả perfectionner de jour en jour leurs instruments, elle a amené les physiciens à rectifier les idées qu'ils professaient depuis Galilée, Kepler et Descartes sur la formation des images dans le microscope. Enfin, elle a doté tous ceux qui s'y sont adonnés d'une incomparable habileté dans le maniement des instruments, dans l'emploi et l'appréciation des objectifs et dans la manipulation des petits objets.

(1) Kützıng, Synopsis Diatomacearum, Halle, 1833, in.80, 7 pl.

(2) J. Pelletan, Le Microscope, son emploi et ses applications, 1 vol. in-8 ${ }^{\circ}$ avec grav. et pl., Paris, 1876. 
Aussi, avions nous raison de dire dans un autre ouvrage (2) que les organismes qui ont été le plus utiles à l'avancement de la science si vaste et si ardue des infiniments petits sont les Diatomées, cette joie et ce désespoir des micrographes, ces pierres de touche de nos objectifs; les Diatomées, pour l'examen desquelles ont été construits les plus parfaits, les plus admirables (et les plus coûteux) des instruments; les Diatomées quui ont fait faire à l'art si difficile de la construction des objectifs plus de progrès, peut-être, que tous les étres réunis de la création. 


\section{HISTOIRE NATURELLE DES · DIATOMÉES}

Quelle est la position systématique des Diatomées, autrement dit, quelle place occupent elles sur l'échelle des êtres?

Nous avons vu, dans un précédent chapître, qu'elles ont élé longtemps ballottées du rẻgne animal au règne végétal, que Leeuwenhoek, 0 . F. Müller, Gmelin, Ehrenberg et d'autres auteurs les classaient parmi les animalcules infusoires, tandis qu'Agardh, Dilwyn, Turpin, de Candolle et, après eux, lous les naturalistes les placèrent parmi les Algues, sauf Ingenlıousz qui, en raison de leur enveloppe siliceuse, les rangea dans le rẻgne minéral.

Aujourd'hui, tout le monde est d'accord pour les placer dans le règne végétal, mais à l'échelon le plus bas et parmi ces êtres qui forment, pour ainsi dire, le passage des plantes aux animaux et qu'on a réunis sous le nom d'Algues unicellulaires.

Singulières plantes, en effet, que ces Algues inférieures dont certaines se meuvent de mouvements qui paraissent volontaires, souvent à l'aide de cils vibratiles semblables à ceux' des Infusoires Flagellés, possèdent un point rouge assimilable à un œil dont il remplit manifestement les fonctions, ont une vésicule pulsatile, organe de circulation ou d'excrétion, dont le mécanisme appartient évidemment à l'animalité.

Aussi, beaucoup de ces Algues inférieures, les Chlamydomonas, les Chlamydococcus, les Gonium, les Pandorina, les Volvox, etc., sont elles réclamées par les zoologistes comme faisant parlie de leur domaine.

Oủ est, en effet, le caractère distinctif, le critérium qui permet d'attribuer ces divers êtres à tel règne plutôt qu'ä tel autre?

En réalité, il n'en pas de certain, ni de complet, mais le plus satisfaisant nous paraîl devoir être cherché dans le mode de nutrition.

La pluparl des végétaux se nourrissent grâce ả la chlorophylle ou matière verte dont ils sont munis, laquelle, sous l'influence de la lumière solaire, agil sur l'acide carbonique de l'air, fixe le carbone pour la nutrition de la plante et rejette l'oxygène. Tous les végétaux ne sont pas pourvus de chlorophylle, les Champignons, par exemple, et, par conséquent, ne 
se nourrissent pas ainsi aux dépens de l'atmosphère; mais on doit, à notre avis, considérer comme des plantes tous les organismes à chlorophylle qui se nourrisssent par ce mode de nutrition exclusivement végélal et que, pour cette raison, on appelle holophytique.

Ce n'est pas qu'il n'existe un certain nombre d'ètres certainement animaux qui contiennent de la chlorophylle. Mais ils se nourrissent, néanmoins, à la manière des animaux : ils ont une bouche et avalent leur proie. La chlorophylle est, chez eux, disposée sous forme d'organes particuliers, assez compliqués, ‘u'on a appelés chromatophores, souvent en siluation et en nombre constants pour chaque espéce. Nous supposons que ces organes constituent chez ces animaux, un appareil de perfectionnement destiné à la fonction respiratoire.

Il peut arriver encore que ces organes chlorophyllés soient, chez ces animaux, doués, pour ainsi dire, d'une vie autonome. Ils ont la constitution histologique d'une cellule, se multiplient même par division ce!lulaire, à ce point qu'on les considère aujourd'hui comme des organismes végétaux parasites de l'animal. Il s.emblerait même que ces parasiles sont nécessaires el que l'animal ne pourrait pas vivre sans les parasites, de mêmeque ceux-ci, ma!gré certaines observations qui tendraient ả prouver le contraire, ne peuvent pas vivie en dehors de leur hôte. Il y aurait là un fait d'adaptation aboutissant à une symbiose par service réciproque. On a même classé ces parasiles parmi les Algues inférieures, les Palmellacées, sous les noms de Zoochlorella, Zooxanthella, etc.

Les Diatomées n'ont ni cils vibratiles, bien que certaines se meuvent, ni point rouge oculiforme, ni vésicule contractile, mais elles sont pourvues d'un endochrốme formé par une variété jaune ou brune de la chlorophylle et disposé en plaques ou en grains, c'est à dire en chromatophores, dont le nombre et la situation sont constants pour chaque espèce Elles se nourrissent uniquement grâce à celte chlorophylle modifiée, qu'on a appelée diatornine et qui agit ả la lumière solaire comme la chlorophylle des plantes vertes, décompose l'acide carbonique de l'air dont le carbone est utilisé par la Diatomée, et rejette l'oxygène. Leur mode de nutrition est donc entièrement holophytique ; elles ne possẻdent, d'ailleurs, aucun des organes de l'animalité. Il y a donc lieu de les classer dans le règne végétal et, par exemple, de les rapprocher des Algues inférieures.

La matière colorante qui forme l'endochrome ou les .chromatophores des Diatomées, bien qu'elle soit rarement verte, agit donc sous l'influence de la lumière comme la chlorophylle des plantes vertes. Nous avons dit qu'elle est formée par une modification de la chlorophylle, il serait plus juste de dire qu'elle est composée d'ın mélange, à proportions variables suivant les espèces, de chlorophylle ordinaire et d'une chlorophylle modifiée que M. Paul Petit, dans un intéressant travail qu'il a publié sur ce sujet (1), a désigné sous le nom de phycoxanthine. "Celle-ci est soluble

(1) P. Petst, Brebissonia, 1880. 
dans l'alcool faible, qu'elle colore en jaune, tandis que la chlorophylle n'est soluble que dans l'alcool fort, le chloroforme, etc. La phycoxanthine, que II. Frémy a appelée phylloxanthine, soumise au spectroscope, ne donne que deux bandes d'absorption, l'une dans l'orangé et le jaune, entre les lignes B et C du spectre, l'autre qui éteint toutes les parties de l'indigo ef du violet au delà du milieu de l'espace $\mathrm{E}-\mathrm{F}$. La chlorophylle donne cing bandes, la première dans l'espace $\mathrm{B}-\mathrm{C}$, la seconde à gauche de la ligne $\mathrm{D}$, la troisième au milieu de l'espace $\mathbf{D}-\mathrm{E}$ dans le vert, le quatrième en $\mathbf{E}$, et la dernière éteint toute la partie lumineuse du spectre au delà du milieu de l'espace $\mathrm{E}-\mathrm{F}$.

La chlorophylle ou matière verte qui, chez les Diatomées est mêlée à la phycoxanthine, se comporte absolument comme la chlorophylle ordinaire des autres plantes, et les Diatomées vertes telles que les Melosira nummuloides, Navicula rumosissima, etc., agissent elles-mêmes sur la lumière comme la chlorophylle ordinaire. Les Diatomées brunes, au contraire, ne donnent que la large bande $\mathrm{BC}$ de la phycoxanthine et l'étroite bande dans l'espace orangé $\mathrm{C}-\mathrm{D}$ de la chlorophylle. Elles éteignent aussi la partie lumineuse à partir du milieu de l'espace $\mathrm{E}-\mathrm{F}$, comme, la phycoxanthine.

Que les Diatomées agissent sur l'acide carbonique de l'air comme les plantes vertes, c'est un fait qu'il est facile de vérifier. Il est évident, d'ailleurs, qu'étant des plantes aquatiques, elles vivent à l'aide de l'air dissous dans l'eau qu'elles habitent. Si l'eau cesse d'etre aérée, les Diatomées meurent, de même que si elle renferme des gaz de putréfaction. Il est facile de répéter avec elles la fameuse expérience de Saussure, comme nous l'avons fait, il y a quelques années.

Nous avons expérimenté sur une récolte pure et fraîche de Fragilaria capucina formant une masse à peu près de la capacité d'un dé à coudre. Nous avons vidé toute la récolte dans une petite éprouvette en verre, - un tube à essai - et nous avons achevé de remplir complẻtement avec de l'eau pure de rivière; puis, nous avons placé dans l'ouverture du tube un petit bouchon de liége, assez petit pour laisser un certain espace entre sa périphérie et les parois internes du tube. Celui-ci a alors été rempli de manière à ce qu'il débordât. Puis, le fermant avec le pouce, sans emprisonner de bulles d'air, nous l'avons retourné sur un vase plein d'eau, et débouché.

Dans cette position renversée, les Diatomées, en hy vertu de leur poids, tomberaient peu à peu et, sortant du tube, viendraient se répandre au fond du vase. Mais le petit bouchon, à cause de sa légèreté spécifique, s'éléve doucement dans le tube et vient s'arrêter vers la partie supérieure en remontant les Diatomées qui restent ainsi suspendues en grande quantité en haut du tube, reposant sur le bouchon (1).

(1) On n'aurait pas besoin de ce petit artifice en opérant sur une cuve à 
L'appareil est alors consolidé au moyen d'un support à pince et placé á une vive lumière. Il n'est pas utile de l'exposer à la lumière solaire directe : le phénomène est plus rapide, il est vrai, mais l'eau, en s'échauffant, perd une certaine quantité de l'air qu'elle dissolvait, air qui vient s'accumuler au sommet du tube et trouble les résultats. En plaçant l'appareil à la lumière vive, près d'un objet éclairé par le soleil, on voit peu à peu de la masse des Diatomées se dégager de fines bulles de gaz qui se rassemblent au sommet du tube. En quelques heures, on obtient ainsi un volume de gaz occupant un centimètre cube, si les Diatomées sont bien vivantes, la lumière solaire active et l'eau employée bien fraîche.

Ce gaz est de l'oxygène, avec une petite quantité d'air en nature rendu par l'eau. Il est facile d'en faire la preuve. On retourne le tube, en le tenant fermé avec le pouce, et, au moment où l'on soulève le pouce, on insinue dans la petite atmosphère gazeuse une allumette éteinte et présentant un point en ignition. Celle-ci se rallume aussitôt. Ou bien, on fait passer la bulle de gaz dans un eudiométre et, après y avoir ajouté un volume double d'hydrogène pur, on fait jaillir dans le mélange une étincelle électrique. Le mélange brùle grâce à l'oxygène, et il ne reste plus au sommet de l'éprouvette qu'une toute petite bulle formée d'azote et de l'un ou l'autre des gaz, hydrogène ou oxygène, en excès.

Il n'y a, du reste, rien d'étonnant à voir cette chlorophylle brune des Diatomées, ou ce mélange de produits dérivés de la chlorophylle, se comporter avec l'air, sous l'influence de la lumière, comme la chlorophylle ordinaire des plantes vertes. Nous connaissons, en effet, un grand nombre de végétaux, dits à feuillage coloré, chezlesquels la chlorophylle a subi des modifications diverses, souvent très analogues à la matiẻre colorante jaune ou brune des Diatomées. Tout le groupe des Algues marines appartenant aux Fucacées est coloré par' une chlorophylle d'un brun verdâtre très foncé et tout le groupe des Floridées est coloré en un rouge parfois très vif. Les Phanérogames présentent aussi des espèces dont les feuilles sont colorées en toutes les nuances du vert ; un grand nombre, comme les Coleus, les Begonia, les Alternanthera, les Caladium et mille autres, ont toutes les nuances du vert, du rouge et du blanc; tout le monde connaît les Hêtres et les Noisetiers au feuillage pourpre, les plantes à feuilles panachées de blanc ou de jaune, le Perilla nankinensis à feuilles presque noires, etc. Tous ces mélanges de produits dérivés de la chlorophylle verte agissent comme elle sur l'atmosphère en décomposant l'acide carbonique de l'air, fixant le carbone et rejetant l'oxygène.

Enfin, ces modifications se produisent sonvent sur une mème plante : par exemple, quand les feuilles deviennent jaunes ou brunes en automne, ou meme d'un rouge de sang, comme dans la Vigne vierge.

Les Diatomées, se nourrissant comme les plantes vertes et exclusive-

mercure et en faisant passer les Diatomées et l'eau dans une éprouvette pleine de mercure et renverséc sur la cuve. 
ment comme elles, appartiennent donc évidemment au règne végétal; il reste à déterminer le groupe de végétaux dans lequel on doit les ranger.

Incontestablement, elles appartiennent à la classe des micro-organismes et doivent être placées à côté des Algues unicellulaires.

Ce sont, en effet, des organismes unicellulaires. Leur corps entier n'est, au point de vue histologique, qu'une cellule. Et quand M. J. Deby dit, dans l'Introduction de ce livre, qu'il regarde les Diatomées comme des êtres pluricellulaires, il veut exprimer que l'organisme qu'on a appelé frustule, et que l'on considêre ordinairement comme formant à lui tout seul toute la Diatomée, n'en est, au contraire, qu'un des éléments, la Diatomée se composant d'une agrégation de ces frustules unicellulaires, comme le Volvoce se compose d'une agrégation de monades formées d'une seule cellule. C'est à dire que les Diatomées sont plurifrustulaires, mais le frustule n'est qu'une cellule. Il présente une enveloppe cellulaire ou cuticule, un protoplasma, un noyau et un nucléole. De plus, il est muni de chromatophores diversement constitués.

C'est là la composition d'une simple cellule. Néanmoins, on a contesté que le frustule même des Diatomées puisse être une cellule simple. Certains auteurs se sont fondés, dans cette discussion, sur la complexité de ces organismes, dans lesquels on trouve des différenciations trop considérables, selon eux, pour qu'on puisse les attribuer à un simple élément cellulaire. D'autres se sont appuyés surtout sur l'existence de deux protoplasmas, le premier, protoplasma ordinaire ou incolore, et le second, protoplasma coloré.

Nous pensons qu'aujourd'hui cette thèse n'est plus soutenable: le protoplasma coloré, l'endochrôme, n'est formé que par les chromatophores dont on constate l'existence, non senlement chez les Diatomées, mais encore chez un grand nombre d'autres cellules, d'abord dans toutes les cellules vertes des plantes, puis cliez beaucoup d'organismes reconnus comme unicellulaires. Tels sont beaucoup d'Infusoires flagellés, comme les Dinobryiens, par exemple, qui possèdent de chaque côté de la cellule unique qui constitue leur corps, une plaque colorée en jaune brunâtre, comparable aux plaques d'endochrôme de beaucoup de Diatomées, comme les Navicula.

Quant à la complexité de la cellule qui forme le corps des Diatomées, elle ne saurait non plus présenter un argument contre l'attribution de ces petites plantes à la classe des organismes unicellulaires. Celte complexité, d'ailleurs, n'est pas si grande qu'on peut le supposer ; elle réside en entier dans la faculté dont est douée la cellule diatomée de sécréter une carapace siliceuse qui double la membrane cellulaire d'une coucheminérale indestructible et qui est pourvue de détails de structure, stries, perles, diaphragmes, pores, nodules, etc. fort compliqués, mais constants pour chaque espèce.

Mais, quand on compare ces détails de structure à ceux qui sont réalisés 
par de simples différenciations des éléments cellulaires, chez d'autres micro-organismes, on reconnait qu'ils n'ont, bien qu'extrẻmement curieux et délicals, qu'ıne importance tout à fait secondaire, auprẻs de ceux que présentent les Infusoires, les Myxosporidies et beaucoup d'autres organismes unicellulaires.

Avec les simples éléments cellulaires qui les constituent, les Infusoires peuvent se créer, par des différenciations de ces éléments, des organes locomoteurs, une bouche, un intestin, un anus, un appareil contractile, une cuticule parfois munie d'organes urticants ou trichocystes comparables à ceux des Zoophyles; on les voit se constituer des carapaces ou des coques de formes variées, voire des coquilles calcaires ou siliceuses dont les Acanthomètres, les Polycystines, les Foramifères nous offrent d'admirables exemples.

Et quant au frustule siliceux des Diatomées, ce frustule composé de deux valves, dont la jointure est formée par une bande connective, n'est-ce pas un détail qui rappelle complètement la constitution des Psorospermies, (lesquelles ne représentent qu'une phase reproductrice des Myxosporidies) composées aussi de deux valves maintenues par un ruban connectif, valves qui s'écàrtent à cerlains moments pour mettre a nu le protoplasma intérieur en vue d'une conjugaison avec un aulre organisme semblable? C'est le même phénomène qui se passe chez les Diatomées dans les cas, assez peu nombreux, où la conjugaison a été reconnue chez elles; avec celle différence, tout à l'avantage des Psorospermies, que la bande connective n'a, chez les Diatomées, qu'un rôle passif, celui de protéger les valves de nouvelle formation dans la multiplication par voie asexuelle ou division cellulaire, tandis que chez les Psorospermies les filaments connectifs agissent comme de véritables grappins ou rétinacles pour maintenir l'organisme conjoint pendant l'acle de la reproduction sexuelle ou conjugaison.

Chez les Diatomées, le seul organe de fixation pendant la conjugaison est un mucilage dans lequel les deux cellules sont enveloppées pendant toule la durée du phénomène ; tandis que, chez les Myxosporidies l'état, de mucilage ou de myxome est une véritable phase biologique, phase vègétative pendant laquelle se forment, dans le myxome, les Psorosperinies qui se conjugueront plus tard librement (1).

Nous ne voulons pas pousser plus loin qu'il ne faut cette comparaison des Diatomées arec cerlaines phases du cycle biologique des Sporozoaires, comparaison qui cependant conduit à des rapprochements très curieux. Nous voulons seulement établir que la complexité de la cellule diatomée et les différenciations qu'elle subit, ne sont point un argument contre l'opinion, aujourd'hui admise par la grande majorité des naturalistes, sur la nature unicellulaire des Diatomées; que, bien au contraire, les ana-

(1) G. Balbiavi. - Lecons sur les Sporozoaires, recueillies par le $\mathrm{D}^{\mathrm{r}} \mathrm{J}$, Pelletan. 1 rol. in-8, avec figures et planches, Paris, 1885. 
logies singulières que présentent ces'différenciations et les phénomènes dont celles-ci sont l'objet ou le moyen, ne peuvent que confirmer cette opinion, s'il en était besoin.

Si nous continuons, en effet, cette étude des Diatomées au point de vue biologique, nous arrivons à constater chez elles une série de phénomèries très intéressants et qui se rapprochent complètement de ce qu'on observe chez d'autres groupes d'organismes unicellulaires. Les uns sont relatils à la .constitution même des Diatomées, les autres à leurs différents modes de reproduction.

M. J. Deby expose, dans l'Introduction du présent ouvrage, la struccture anatomique, parfois si compliquée, de la cellule diatomée elle-méme, le frustule. Mais nous ne pensons pas que l'organisme soit tout entier constitué par cette cellule ou ce frustule. Il y a encore un autre élément sur lequel plusieurs auteurs ont déjà appelé l'attention des naturalistes, mais auquel on n'a pas, à notre avis, accordé toute l'importance qu'il peut avoir. Nous voulons parler de ce que M. Matteo Lanzi a appelé le thalle des Diatomées.

On a vu que M. Deby donne ce même nom de thalle à l'ensemble des frustules qui constituent une entité biologique Algue diatomée, et forment l'appareil végétatil de cette Algue, appareil végétatif qui peut rester cohérent, comme dans les Diatomées filamenteuses, ou se désagréger au fur et à mesure, comme dans les Diatomées diffluentes.

Cette vue nous paraît fort juste, mais il y a quelque chose de plus. Chez un très grand nombre d'espéces, on a reconnu facilement l'existence d'une substance servant de support ou d'enveloppe extérieure aux frustules cellulaires et qui a parfois une forme bien définie. Par exemple, beaucoup de Diatomées, comme les Gomiphonema, sont portées par un pédicelle, c'est-à-dire par un filament, incolore, de consistance un peu mucilagineuse et doué de certaines propriétés autonomes, par exemple, de la propriété de prendre cette forme filamenteuse et de croître en longueur en se divisant et se subdivisant par bi-partitions dichotomiques. Il est anhiste, mais il n'est pas amorphe. C'est là le " thalle " pour M. M. Lanzi ; nous l'appellerons thalame pour éviter toute confusion.et ne rien préjuger.

D'autres fois, ce thalame est constitué par des tubes transparents, géla. tineux, d'un diamètre qui varie peu et dans l'intérieur desquels les frustules cellulaires s'entassent et se disposent dans une situation constante. La matière qui constitue le thalame tubulaire de ces espèces est donc douée de la propriété autonome de croître en longueur en affectant toujours une disposition en tube. Ce n'est donc pas une matière inerte.

D'autres fois encore,le thalame ne présente pas une forme aussi nettement définie de pédicelle ou de tube, mais constitue un amas mucilagineux et transparent dans lequel sont englobés des frustules plus ou moins nombreux ou un frustule unique. C'est le mucus matricalis des auteurs. Il 
forme une masse amorplie, une phytoglée, composée d'une substance glaireuse semblable à celle dont sont enveloppées beaucoup d'Algues inférieures, Palmellacées, Nostocacées, Volvocinées, etc.

C'est dans une couche de celte glaire, qui ordinairement devient plus abondante et foisonne pour la circonstance, que s'opèrent les phénomènes de la reproduction sexuelle et de la formation des germes observés par M. Matteo Lanzi. Il peut même arriver que les thalames á forme définie, comme le pédicelle des Gomphonema, se transforment en une large expansion hyaline aux bords de laquelle, le phénomène de la reproduction accompli, poussent les pédicelles filamenteux qui portent les jeunes frustules (1).

Chez beaucoup d'espéces, le thalame mucilagineux est moins évident. Néanmoins, quoique réduit à une couche très mince, et peu visible, d'ailleurs, en raison de sa grande transparence, il n'en existe pas moins. Ainsi, chez toutes les Diatomées qui affectent la forme filamenteuse, comme les Melosira, les Himantidium, et une foule d'autres, les frustules demeurant accolés les uns aux autres au fur et à mesure qu'ils se forment par des divisions successives, il est certain que ces frustules restent adhérents parce qu'ils sont enduits, notamment sur leurs faces de contact, d'une couche extérieure agglutinative, qui est formée par la matière hyaline du thalame.

La meme observation peut se faire pour les Diatomées dont le frustule est triangulaire, comme les Meridion et les Licmophora, frustules qui, restant accolés après des divisions successives forment, non seulement des cercles, mais des figures spirales comme une vis.

Et, de même encore pour les espèces dont les frustules restent accolés par un de leurs angles, comme les Tabellaria. En général, chez celles-ci, il est assez facile de voir la petite masse ou coussinet de substance hyaline qui sert à fixer l'angle d'un frustule à l'angle du frustule voisin.

Enfin, mème chez les espèces dont les frustules paraissent libres e $i$ isolés, il est certain qu'il existe sur toute la surface extérieure une couche de substance transparente, ordinairement trẻs mince, mais dont il est néanmoíns facile de vérifier la présence el qui, par exemple, masque partiellement ou complètement à l'œil de l'observateur les détails, stries et sculptures dont est ornée la surface siliceuse des valves qu'elle recouvre. C'est cette couche agglutinative externe qui fixe les Diatomées libres sur les plantes, les pierres, et, en général, tous les corps qui leur servent de support. II faut qu'on la détruise pour pouvoir reconnaitre les détails de structure du frustule.

Nous pensons donc qu'à l'état normal et complet, l'entité biologique qu'on appelle une Diatomée se compose d'un thalame plus ou moins volumi-

(1) Matreo Lan7. - Le thalle des Diatomées (Bull. de la Soc. belge de M/2croscopie, 1880. - Journal de Micrographie, 1880.) 
neux, à forme définie ou à forme indéfinie, et d'un ou plusieurs corpuscules cellulaires ou frustules. Le tout forme le thalle de la plante.

Et nous sommes convaincu que c'est en considérant les caractẻres généraux de ce thalle tout entier, comme on le fait pour les autres Thallophytes, et les modes de fructification, quand on les connaîtra mieux, qu'on arrivera à une classification vraiment naturelle des Diatomées.

Cerlains frustules présentent une structure et un aspect identiques, mais les uns appartiennent à une espéce possédant un thalame évident, filiforme ou tubulaire, tandis que les autres appartiennent à une espèce n'ayant qu'un thalame réduit à. une mince couche péricellulaire. De sorte que si l'on examine les frustules de la première espèce, alors qu'ils sont dépourvus de leur thalame, par suite d'un accident ou par les progrès de l'âge, on est exposé à les confondre avec ceux de la seconde espèce. Et, inversement, un même frustule, suivant qu'il est encore adhérent à un thalame défini ou qu'il en est débarrassé, peut être rangé dans deux espèces différentes.

Quelle importance doit-on donc donner au thalame des Diatomées soit dans la classification, soit dans les phénoménes qui constituent la vie de la plante?

Ce sont là des questions très complexes et auxquelles nous n'avons encore à répondre que d'une manière très peu satisfaisante.

Au point de vue de la classification des espéces, il est évident que les notions tirées du thalame sont de peu de ressources. Quand celui-ci est bien défini, d'une constatation facile dans la majorité des cas, comme les thalames pédicellaires ou tubulaires, il est certain qu'il peut fournir des caractères pour la distinction des espèces. On s'en est servi, en effet, dans les classifications en usage aujourd'hui. Néanmoins, comme nous venons de ie montrer, ces caractères sont dangereux à l'emploi quand il s'agit de déterminer des frustules qui se sont libérés de leur thalame. Et quand celui-ci se réduit à la mince couche pelliculaire qui revêt le frustule de toutes les Diatomées, il est difficile, quant à présent, sinon impossible, d'y chercher des caractères. Nous pensons donc qu'actuellement on a tiré du thalame, au point de vue taxonomique, à peu près tout le parti qui était possible.

Quant au point de vue biologique, l'importance du thalame des Diatomées varie beaucoup avec la constitution qu'on peut lui supposer. N'est-ce qu'une simple matiẻre inérte, destinée à servir le support et d'enveloppe protectrice au frustule, comme celle qui enveloppe beaucoup d'Algues inférieures, les Pálmella, les Nostoc, les Gonium, les Volvox, etc., où elle ne parait remplir qu'un rôle mécanique de protection et de réunion pour les cellules vivant en colonies? S'il en est ainsi, son importance est secondaire. C'est un simple coléoderme, comme l'appelait Brébisson.

Mais, au contraire, remplit-il une fonction plasmatique? Intervient-il à certains moments, comme élément formateur? Est-il, par exemple, com- 
parable à ces masses plasmatiques, mucilagineuses aussi d'apparence, qui constituent la phase végétative des Myxosporodies, masses au sein desquelles se produisent par des différenciations, des polarisations dans ce protoplasma, ces singuliers corpuscules, aux formes si curieuses, qu'on a appelés Psorospermies?

S'il en élait ainsi, la part de cette glaire dans le cycle biologique des Diatomées serait considérable. On pourrail faire un rapproclıement saisissant entr'elles et un grand nombre de Sporozoaires dans lesquels on voit d'abord une masse protoplasmique constituer l'organisme tout entier, masse dans laquelle, plus tard, apparaissent, à la suite de processus plus ou moiıs coinpliqués, des corpuscules à forme définie, forme qui rappelle méme quelquefois celles de certaines Diatomées naviculaires à ce point qu'on a appelés ces corpuscules pseudo-navicelles.

Ainsi, pour fixer les idées, nous rappellerons brièvement l'évolution de ces bizarres Sporozoaires, si bien étudiés par M. Bütschli et surtout par M. Balbiani, et qu'on a désiginẻs sous le nom de Myxosporidies :

Sur les lamelles branchiales de certains poissons, de la Tanche, par exemple, on voit de petites masses arrondies, ou bien allongées en boyau, uquelquefois ramifiées, d'une matière plasmatique ou sarcodique, qui sont douées de mouvements amiboïdes, émetlant mème, dans quelques cas, des expansions ou pseudopodes plus ou moins longs et fins. Souvent, comme on le voit aussi chez certaines Amibes, elles ne sont le siège que de mouvements we fluctuation se propageant tantôt dans un sens, tantôt dans l'autre, et qui se produisent sur place, sans déterminer de progressionde la masse plasmatique, parce que celle-ci repose sur un support solide. Si ces masses étaient flottantes dans un liquide, on conçoit que les ondes de fluctuation, formant comme une vague qui se propage, pourraient leur imprimer un mouvement de translation dans un sens ou dans l'autre, suivant le sens de l'ondulation.

Ces masses sarcodiques grossissent peu à peu, et varient beaucoup de dimension suivant les espèces et la phase de développement, quelques unes ne dépassant pas $8 \mu$, d'autres alteignant jusqu'à $36 \mu$.

Peu à peu, il se développe, dans leur intérieur, des globules d'une matière gुrasse colorée en jaune et des granulations quui prennent le caractère de noyaux et qui se multiplient, grossissent, et, à la suite de transformalions encore mal connues, acquiẻrent une forme elliptique, s'enveloppent d'une membrane solide ou coque transparente et constituent ce qu'on a appelé une psorospermie; ces corpuscules doivent être considérés comme des corps reproducteurs ou spores.

Ces psorospermies, qui correspondent chez les Myxosporidies aux corpuscules que chez d'autres Sporozoaires, les Grégarines et les Coccidies, on a appelés pseudo-navicelles à cause de leur forme naviculaire, sont de petits organismes très compliqués.

La coque est formée de deux valves appliquées l'une contre l'autre, 
comme les deux moitiés d'une coquille de noix, et la ligne de suture est visible quand on examine le corpuscule par la tranche (ou par la face connective, comme on dirait pour une Diatomée). Chaque valve est garnie, tout autour de son bord, d'une fine bande ou ruban tellement appliqué contre ce bord qu'il est impossible de le voir, si ce n'est au moment de la déhiscence de la psorospermie.

De plus, à l'un des pôles du corpuscule et dans son intérieur, se trouvent deux vésicules qui contiennent un filament enroulé ; on peut faire sortir de la psorospermie, à l'aide de certains réactifs, ces deux filaments qui sont deux ou trois fois plus longs que la psorospermie elle-meme. Au dessous de ces deux vésicules sont deux granules brillants, vésicules polaires en expectative, et, au centre du corpuscule, un amas protopla:mique au milieu duquel est un noyau.

Telle est la période végétative de la Myxosporidie de la Tanche, période dans laquelle, au sein d'une masse protoplasmique à peu près amorphe,se forment des corpuscules figurés, très compliqués, mais que l'on peut néanmoins considérer comme de simples cellules constituant les corps reproducteurs et dont la formation inaugure la période reproductrice.

Poursuivons ủonc cette analyse et voyons comment se clôt cette dernière période et se ferme le cycle biologique de ces singuliers organisnes.

A un certain moment, la psorospermie ou spore ayant atteint sa maturité, les rubans ou bandes connectives se décollent du bord des valves, sauf par un point situé au pôle postérieur, et l'on voit alors qu'elles sont très longues et se prolongent en un filament pointu ou arrondi. Deux psorospermies voisines se saisissent, pour ainsi dire, l'une l'autre par leurs filaments et s'accrochent à l'aide de ces organes préhenseurs; ceux ci, faisant alors ressort élastique, amènent la déhiscence des valves de chaque psorospermie qui s'écartent, mettant en liberté le globule protoplasmique intérieur. Que se passe-t-il alors? Sans doute une conjugaison par fusion ou échange des globules protoplasmiques intérieurs; on n'est pas encore fixé d'une manière certaine sur ces phénomènes qui sont très dịfficiles à suivre. Toutefois, on sait que les vésicules à filament spiral se vident, les filaments sortent et se raidissent, contribuant à fixer le corpuscule à la place qu'il occupe, jusqu'à ce que le globule protoplasmique intérieur, à la suite de l'espèce de fécondation qu'il vient de subir, s'anime tout-à-coup de mouvements amiboïdes et sorte de la psorospermie à travers les valves écartées. Or, une fois libre, cette petite masse protoplasmique n'est autre chose qu'une nouvelle Myxosporidie ¿qui va grossir peu á peu et au sein de laquelle vont se passer les mêmes phénomènes que nous avons décrits.

Encore une fois, ncus ne voulons pas établir une assimilation complète des Diatomées et des Sporozoaires, mais nous cherchons si les faits 
que nous venons de résumer brièvement et qui sont relatifs aux Myxosporidies, qui se produisent d'une manière à peu près semblable chez les Sarcosporidies et les Microsporidies, ne pourraient pas nous aider à éclaircir quelques-uns des phénomẻnes que l'on remarque chez les Diatomées, notamment ceux qui ont rapport aux mouvements qu'exécutent ces organismes et aux divers processus de leur reproduction. C'est ce que nous .l'ons examiner maintenant.

\section{§ 2. - MOUVEMENTS DES DIATOMÉES}

Un grand nombre de Diatomées sont douées de mouvements propres, et particulièrement celles dont les frustules affectent une forme allongée ou en nacelle, comme les Navicula, les Pinnularia et beaucoup d'autres. Il ne s'agit pas de ces vibrations moléculaires dont sont douées les très fines particules, même minérales, suspendues dans un liquide et, qu'on appelle mouvement brownien, mais d'une motilité autonome, qui semble mìme spontanée et volontaire. Cette propriété n'a pas peu contribué jadis à faire classer ces espèces parmi les animalcules infusoires.

Si l'on dépose sur le porte-objet une goutte d'eau tenant en suspension plusieurs Navicula vivants, on les voit aussitôt se mettre en mouvement et se diriger tous, comme autant de petites nacelles, dans un sens différent, ce qui prouve que le mouvement n'est pas dù à un courant établi dans le liquide. La translation se fait toujours dans le sens de la longueur du frustule. Souvent, d'ailleurs, le petit corps, après s'être avancé dans un certain sens, s'arrête, comme hésitant, et repart en sens contraire. La plupart du temps, il va, pour ainsi dire, aveuglément, se jetant sur les obstacles qui se trouvent devant lui, et c'est alors que, d'ordinaire, il rebrousse chemin; mais quelquefois, cependant, il parait se détourner, comme par un secret instinct, des corps qui peuvent l'arrêter. Cet effet, peut être dû à une petite différence dans la densité de l'eau qui se trouve un peu condensée, dans une certaine zône autour,des corps immergés, par un effet d'attraction moléculaire ou capillaire.

Les Diatomées qui vivent associées en groupes sous forme de filaments, d'arborisations ou d'éventails peuvent aussi exécuter ces mouvements si, pour une cause quelconque, leurs frustules deviennent libres. Des espèces dont les frustules sont enfermés dans un tube gélatineux se meuvent aussi, c'est-à-dire que les frustules se déplacent dans l'intérieur du tube, ce qui démontre, par parenthẻse, que, chez ces espẻces au moins, le thalame est réellement tubuleux, ne contenant dans son intérieur que de l'eau ou une matière beaucoup moins dense que celle dont est formée la paroi.

Une des espéces les plus célẻbres "parmi les Diatomées mobiles est le Bacillaria paradoxa, l'ancien Vibrio paxillifer de 0.-F. Müller; cette espèce est composée de plusieurs frustules en bâtonnets, disposés parallè- 
lement les uns aux autres de manière à former une sorte de tablette quadrangulaire. Bientôt, le premier bâtonnet glisse le long du second, parallẻlement à sa direction, et s'arrête quand il ne touche plus la tablette que par une de ses extrémités. Puis, le second bâtonnet, imitant le premier, va le rejoindre en glissant sur le troisième; puis, le troisième va se placer sous le second, le quatrième sous le troisième, et ainsi de suite jusqu'à ce que tous les frustules se soient déplacés. La tablette s'est ainsi avancée latéralement de toute sá largeur. Alors, le premier bâtonnet yecommence son mouvement, mais en sens inverse, et reprend la position qu'il occupait d'abord; le second le suit, puis le troisième, et tous les autres. Et le phénomène se reproduit ainsi indéfiniment.

Depuis bien longtemps on cherche l'explication de ces singuliors mouvements des Diatomées. La première idée a laquelle on a dû songer naturellement, en voyant tant d'Infusoires se mouvoir à l'aide de cils vibratiles, a été d'admettre aussi chez les Diatomées la présence de cils vibratiles plus ou moins fins. Certains auteurs ont aflirmé les avoir vus, soit naissant à la surface du frustule, soit sortant du protoplasma intérieur par des pores de la carapace.

Malheureusement, aucun observateur moderne n'a pu réellement constater l'existence ni le mouvement de ces appendices. On a bien vu à la surface de certains frustules une croûte liyaline plus ou moins irrégulière et présentant en divers endroits comme de petites pointes. Mais ces pointes, plus ou moins longues, outre qu'elles n'existent pas sur tous les frustules d'une mème espèce et qu'elle n'ont pas de positions constantes, n'ont jamais manifesté de mouvements ciliaires ni vibratiles. Aussi, les considère-t-on seulement comme des produclions parasitaires.

L'explication par les cils vibratiles étant généralement repoussée, on a dû, non moins naturellement, penser à l'endosmose, car on sait que l'ère de l'endosmose a succédé dans la science à l'ère des cils vibratiles. On a donc dit que les mouvements des Diatomées sont produits par descourants endosmutiques qui s'établissent, par les pores et les fentes que présentent le frustule, entre l'eau ambiante et le liquide protoplasmique intérieur.

C'est encore à cette explication qu'on s'arrête le plus généralement aujourd'hui et c'est, à notre avis, un tort, car cette explication a un défaut grave : elle n'explique rien du tout. Des " courants endosmotiques ", c'est un mot dont on se paie pour ne pas être obligé de chercher autre chose. Cela ne serait une explication que si l'on montrait comment ces courants agissent pour produire un certain mouvement, et, un instant après, pour produire le mouvement inverse. Des courants endosmotiques ne peuvent ainsi changer de sens d'un moment à l'autre.

Aussi, beaucoup d'auteurs, sentant tout ce que cette prétendue explication a d'incomplet, ont cherché à étudier de plus près les mouvements des Diatomées, et nous ne pouvons citer ici tous les raisonnements plus ou moins compliqués qui ont été mis en avant pour arriver à une explication 
qui explique. Nous devons toutelois citer M. Hamilton L. Smith qui a employé le procédé bien connu des milieux colorés. Il a répandu dans l'eau oủ nageaient des Pinnularia, espéce à forme naviculaire, de l'indigo tel que l'emploient les aquarellistes. Cette matière colorante n'est pas, on le sait, réellement soluble dans l'eau, mais s'y répand en particules extrêmement fines qui donnent à l'eau une teinte bleue fort intense, et que l'on distingue au microscope.

Il a vu ainsi que quand la Diatomée s'avance dans un certain sens, les particules d'indigo s'amassent au niveau du nodule central des valves, en formant une pelite masse arrondie tournant sur elle-même, comme si elle était soumise à un " petit jet d'eau s sortant de la valve à cet endroit. Chacune de ces petites sphères tourbillonnantes, après avoir acquis un certain volume, se désagrège et les particules qui la composent sont chassées le long des valves, d'avant en arrière, pour s'accumuler derriẻre l'extrémité du frustule qui, dans la progression, est en arrière.

Si le mouvement de la Diatomée se renverse, les particules d'indigo sont chassées en sens contraire, c'est-à-dire toujours de la partie médiane vers l'extrémité du frustule qui est en arrière, dans le mouvement. Ces particules vont donc toujours comme si elles étaient soumises à un courant allant d'avant en arrière et se renversant, par conséquent, lorsque le mouvement se renverse.

C'est un résultat auquel, en somme, on devait s'attendre,mais c'est un effet, et l'on ne connait toujours pas la cause.

Mais cette expérience a permis de conslater, d'une manière bien nette, l'existence, à la surface du frustule, d'une couche d'une substance hyaline qui ne se laisse pas pénétrer par les grains d'indigo et forme une zone incolore entre la surface du frustule et le liquide ambiant chargé de particules bleues. C'est ce que nous avons appelé le thalame.

M. H. L. Smith a même fait la contre épreuve, c'est-à dire qu'il a coloré la couche hyaline en une nuance assez vive de manière à la mettre encore mieux en évidence. En ajoutant, dans l'eau où nagent les Pinnularia, une très petile quantité de rouge d'aniline soluble, il a vu la matière colorante se concentrer dans la couche hyaline périfrustulaire et la colorer en rose vif, alors que l'eau de la préparation était elle-même à peine teintée.

Nous avons fait la mème expérience avec de l'éosine, matière colorante pour laquelle la substance hyaline périfrustulaire présente une affinité aussi grande que pour le rouge d'aniline. L'observation de M. H. L. Smith est peu exacte quant au mouvement des particules en suspension dans le liquide, mais la coloration rose se produit immédiatement, et, comme l'a constaté aussi M. H. L. Smith avec le rouge d'aniline, le mouvement de la Diatomée est instantanément arrêté.

On aurait donc ainsi démontré, à l'aide d'un réactif colorant du proloplasma, l'existence d'une couche extérieure de protoplasma, invisible dans les conditions ordinaires, a cause de sa minceur et de sa transparence. - 
Cette couche a, d'ailleurs, été reconnue dans un assez grand nombre de cas, et il est possible que ce qu'on regarde souvent comme une production parasitaire sur certains frustules ne soit que cette couche accidentellement épaissie de protoplasma externe.

Protoplasma externe, tel est, en effet, le nom qu'ont donné plusieurs auteurs à celte couche hyaline périfrustulaire. Or, cetle couche, avons nous dit, c'est le thalame du frustule.

Pour nous, toutes les Diatomées vivantes et complètes possèdent celte couche plus ou moins épaisse et régulièrement disposée. Si certains frustules libres ne la présentent pas, c’est qu'ils l'ont perdue, comme la psorospermie sortie de sa gangue myxosporidique, comme la graine sortie du fruit, comme la pomme tombée du pommier.

Mais si les frustules sont revêtus d'une couche de nature protoplasmique, n'est-il pas possible que cette couche soit douée, comme l'est tout protoplasma, de mouvements propres, de contraction, de dilatation, d'ondulation, comme on en voit dans beaucoup de cellules, dans les Amibes, dans les masses myxosporidiques?

Et, si à la surface d'un frustule naviculaire, c'est-à-dire facilement mobile, le thalame protoplasmique éprouve un mouvement de contraction ou d'ondulation, ce mouvement ne déterminera-t-il pas la progression du léger frustule flottant au sein du liquide, dans un sens opposé, et le rejet des particules solides qui viennent le toucher, sur les côtés ou vers l'arrière?

N'est-ce pas, d'ailleurs, un phénomène presque constant dans les mouvements protoplasmiques que ces changements subits dans le sens du mouvement? - Quand on observe la marche des granulations dans les pseudopodes d'une Amide, ne les voit-on pas entraînées d'abord dans un certain sens par le courant protoplasmique, s'arrêter, puis rétrograder, le courant qui les emporte ayant changé de sens? Ces mouvements d'ondulations, comme une vague qui va et vient, ne sont-ils pas caractéristiques de certains organismes formés presqu'uniquement d'une masse de protoplasma, comme beaucoup de Rhizopodes, les Pelomyxa, certains Rhizoflagellés, etc.?

N'est-ce pas, chez certains d'entr'eux, comme chez les Acanthomètres, le protoplasma extérieur, entourant le squelette minéral, qui est chargé d'accomplir tous les mouvements dont est doué l'animal?

Et la preuve que ce serait bien à leur thalame protoplasmique, c'est-à-dire au protoplasma extérieur qui les entoure, que les Diatomées à frustules locomoteurs devraient leurs mouvements, c'est que si l'on détruit ce protoplasma, le désorganise, le coagule, par exemple, en le traitant par une matière colorante qui contracte avec lui une combinaison, immédiatement les mouvements de la Diatomée sont paralysés. Le protoplasma externe et nu, avide du réactif colorant contre lequel il n'est pas protégé, se combine aussitôt avec lui, se colore et, tué, perd sur l'heure ses propriétés; - et cela, bien avant que le proloplasma intérieur du frustule, défendu par 
la carapace siliceuse, soit le moins du monde atteint par le réactif, et bien avant même que l'eau ambiante ait pris de coloration sensible.

Évidemment, les mouvements des Diatomées ne sont pas dus à ces problématiques courants d'endosmose et d'exosmose; il paraîtrait bien plus raisonnable de les attribuer à une couche protoplasmique entourant les frustules, couche qui est souvent assez abondante et douée de mouvements actifs assez énergiques pour imprimer aux frustules eux-mèmes une impulsion à sens variable, mais toujours dans la direction où l'élément ambiant offre la moindre résistance.

Telle est la conclusion à laquelle nous amènent l'élude de ces mouvements et la comparaison que nous avons faite entre les Diatomées et certains organismes voisins, choisis particulièrement chez les Sporozoaires.

Il fảut reinarquer que cette conclusion nous ramène presqu'à l'ancienne conception des cils vibratiles. En effet, on est d'accord aujourd'hui pour regarder les cils vibratiles des organismes qui en sont doués comme des dépendances du protoplasma, pour ainsi dire comme des fins pseudopodes qui seraient constants dans leur forme. Par conséquent, les mouvements ciliaires et les mouvements protoplasmiques proprement dits sont de mème nature et ont la même origine.

La matière mucilagineuse qui recouvre les frustules des Diàtomées est, comme les substances albuminoïdes, soluble dans la potasse caustique. D'aprẻs le prof. H. L. Smith, quand on traite une Diatomée vivante par la potasse, sous le microscope, en déposant une goutte de la solution alcaline sur le bord du couvre-objet, aussitôt que la solution atteint le frustule, la matière mucilagineuse se dissout et les deux valves du frustule s'écartent, s'ouvrant comme une boîte.

1 faut donc en conclure que cette couche hyaline contribue a maintenir les deux valves en contact. Elles s'écartent sous la pression du protoplasma intérieur qui se gonfle peut-être en s'imbibant, par endosmose, de la solution alcaline; M. H. L. Smith pense que c'est plutôt par une pression naturelle du protoplasma contre les parois internes des valves, pression qui n'est plus contrebalancée par la présence et la pression en sens contraire de la matière hyaline externe.

D'après cet auteur, ce serait cette pression, tant interne par le protoplasma, qu'externe par la gaine hyaline, qui déterminerait les mouvements du frustule. De plus, dans les formes naviculées, dont les extrémités sont semblables et plus ou moins pointues, cette pression déterminerait des mouvements, tantôt dans un sens, tantôt dans l'autre, mouvements de va et vient, puisque le frustule est semblable par les deux bouts. Et alors, la matière mucilagineuse, en s'accumulant, se trouverait prendre une forme tubulaire en raison des mouvements alternatifs de la Diatomée dans la glaire. Telle serait l'origine des tubes hyalins dans lesquels sont contenus, et se meuvent, les frustules de certaines espẻces.

Dans les espèces, au contraire, dont le frustule n'est pas semblable à 
ses deux extrémilés, et a, par exemple, plus ou moins la forme d'un coin, la pression de la matière hyaline chasserait toujours le frustule dans le même sens, la base 'du coin en avant, de sorte que cette matière hyaline s'accumulerait toujour's en arrière de la pointe du coin où elle formerait les pédicelles ou stipes que l'on constale chez certaines de ces espèces, comme les Gornphonema.

C'est lả une vue de l'esprit, ingénieuse, comme toutes les conceptions du savant diatomiste américain, mais à laquelle nous pensons qu'on peut faire bien des objections.

\section{§ 2. - MULTIPLICA'TION DES DIATOMÉES}

Les Diatomées, organismes unicellulaires, se multiplient comme tous les organismes unicellulaires : $1^{\circ}$ par division ou fissiparité, mode asexuel ; $2^{\circ}$ par conjugaison, mode sexuel.

Multiplication par fissiparité. - On connaît le phénomène de la division cellulaire; les détails qui l'accompagnent ont été, dans ces dernières années, étudiées avec beaucoup de soin, par un grand nombre d'auteurs, sur des cellules des tissus, et l'on s'accorde aujourd'hui à admettre que le processus très compliqué de la division des cellules a pour origine la division du noyau et de ses éléments. C'est ce qu'on appelle maintenant le phénomène de la karyokinèse.

Nous n'avons pas á insister ici sur les modifications histologiques que subissent, pendant la division, les divers éléments des cellules, nous devons seulement indiquer la marche générale du phénomène tel qu'il se produit sur la cellule diatomée, et ses résultats.

La cellule se compose d'un utricule primordial, c'est-à-dire d'une masse protoplasmique contenant, au centre, un noyau, et au centre de celui-ci, un nucléole; puis un endochrome disposé à la partie superficielle en amas diversement constitués et distribués, formant les chromatophores; enfin, des gouttelettes grasses, plus ou moins nombreuses.

L'utricule primordial est recouvert d'une membrane ou cuticule, ordinairement chargée de silice et élaborée par le protoplasma aux dépens des matériaux qui lui sont fournis par le liquide ambiant.

Si celte enveloppe minéralisée, résistante, était homogẻne et continue autour de la cellule, on comprend que la division ne pourrait pas se faire à moins de s'accompagner de la rupture de cette espéce de kyste.

Mais nous savons que la carapace siliceuse est formée de deux valves dont l'une, dans les conditions ordinaires, est un peu plus petite que l'autre. Ces deux valves ne sont pas soudées par leurs bords, comme les deux moitiés d'une coquille de noix, mais elles sont emboîtées. L'une et l'autre sont munies, sur tout leur pourtour, d'une membrane qui se silicifie plus ou 
moins, les connectifs, zones ou handes connectives. L'une des bandes connectives, celle qui borde la plus petite des deux valves, entre dans l'autre qui la recouvre comme le couvercle d'une boite. - Il en résulte que quand la cellule intérieure augmente de volume en se doublant, l'enveloppe siliceuse s'agrandit par l'éloignement des deux valves dont les connectifs glissent l'un sur l'autre. D'ailleurs, ces connectifs peuvent s'allonger euxmêmes par leur bord, afin de se recouvrir toujours et de ne pas laisser de solution de continuité.

Pendant que les deux valves s'éloignent l'une de l'autre, poussées qu'elles sont par l'utricule primordial qui s'agrandit dans l'intérieur du frustule, le noyau s'allonge et commence à se diviser en deux, chacune des parties emportant un nucléole provenant de la division en deux du nucléole primilif. En mėme temps, le protoplasma périphérique; avec l'endochrome

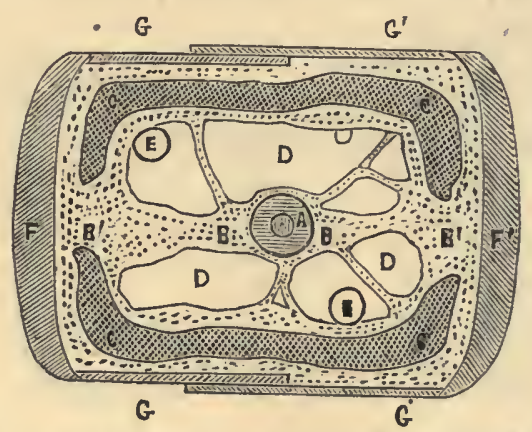

Fig. 14. - Cellule diatomée (Navicule), d'après M. J. Deby.

$\mathrm{BBB}^{\prime} \mathrm{B}^{\prime}$, proteplasma dont une couche entoure la cellule (utricule primordial); A, noyau et nucléole ; C, C, chromatophores; E, E, gouttelettes d'huile; $D$, cavité centrale ; $F, F^{\prime}$, valves; $G, G, G^{\prime}, G^{\prime}$, connectifs.

qu'il limile, s'étrangle dans la zône médiane, sous les connectils prolongés, et la cellule tout entière commence à se diviser en deux autres.

Cette division, se poursuivant, devient bientôt complète et l'on a deux utricules primordiaux ou deux cellules accolées, ayant chacune son noyau, son protoplasma, ses chromatophores, comme la Diatomée primitive. Alors, sur les deux surfaces en conlact se forment deux valves nouvelles, adossées, qui se silicifient bientôt; les bandes de conneclifs qui se recouvrent et débordent les jeunes valves ne tardent pas à se résorber, les deux frustules deviennent libres ef l'on a deux individus au lieu d'un. Un peu plus lard, il se forme dans chaque frustule, le long du bord de la nouvelle valve, un connectif qui glisse sous le connectif de la valve ancienne, et la structure initiale est rélablie dans les deux individus.

Il faut remarquer que, suivant ce processus, tout frustule posse̊de deux valves qui sont d'âge différent, une ancienne et une nouvelle, et qui ne 
sont pas de mème grandeur, la valve nouvelle toujours emboitée dans le connectif de la valve ancienne et, par conséquent, plus petite (1).

Il en résulte, si, comme cela est très probable, la division se fait toujours de la mème manière, que si la taille de la Diatomée s'amoindrit par des divisions successives, il persiste à chaque division un frustule qui maintient la taille primitive, laquelle est réglée par la valve ancienne qui persiste et assiste en témoin à toutes les divisions que subit le frustule qu'elle recouvre.

Ainsi, dans la figure 14 ci-dessus, la cellule représentée par le dessin ne donnera jamais naissance qu'à deux sortes de frustules. Ceux dont la taille est réglée par la grande valve emboitante $\mathrm{F}^{\prime}$, (le frustule de droite, fig. 16) identiques au frustule initial, et ceux dont la taille est réglée par la petite valve emboitée $F$, (le frustule de gauche). Chaque division laisse donc toujours un frustule identique à celui qui se divise et produit un frustule plus petit.

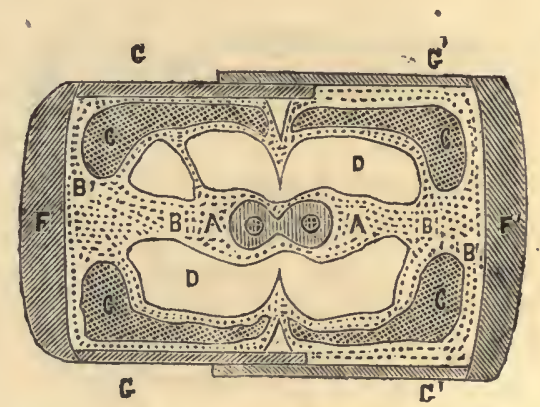

Fig. 15. - Cellule diatomée, commencement de la fissiparité. (d'après M. J. Deby').

Mèmes lettres que pour la figure précedente.

De sorte que si ces frustules " plus petits » deviennent de plus en plus petits, il persiste toujours des individus qui conservent la taille primitive, et il en est qui ont toutes les grandeurs intermédiaires.

Malgré les particularités relatives à la formation et à l'emboitement des connectifs,il est certain que ce phénomène de dédoublement du frustule des Diatomées n'est rien autre chose qu'un processus de division cellulaire

(1) «Il découle de ceci, dit M. J. Deby, que dans la majorité des genres où les connectifs sont de la largeur exacte des valves, ou bien sont même in. férieurs en diamètre à celles-ci, toute déduplication doit amener une diminution des dimensions du frustule nouveau équivalente au double de l'épais. seur d'un connectif. L'épaiss sur de ce dernier étant connue, on peut même, à priori, déterminer la taille qu'aura la descendance d'un frustule quelconque après un nombre de déduplications délerminé. ”

Cette loi de M. Deby n'amène pas un décroissement de la taille aussi rapide que l'indiquerait la raison mathématique de cette progression, parce qu'elle est troublée par la continuation du dédoublement dans les cellules anciennes ou de première génération, comme nous l'expliquons plus haut. 
telle qu'on l'observe dans les cellules des tissus et chez les organismes unicellulaires. Les Diatomistes n'aiment pas que l'on compare les Diatomées à quoi que ce soit dans le monde; pour la plupart d'entr'eux au moins, ce sont des êtres à part dans la création et qui n'ont point d'analogues. Il est bien évident que c'est là une erreur, et quant ả ce phénomène de dédoublement ou déduplication, c'est certainement un fait semblable ả la division fissipare ordinaire qu'on observe chez tous les protophytes comme chez tous les protozoaires. La division se fait en suivant un plan longitudinal et les particularités qu'elle présente dans sa marche sont régis par la forme spéciale, la structure valvaire et la carapace résistante propres aux Diatomées. Dans aucun cas, du reste, ces particularités ne sont plus singulières ni plus compliquées que celles qu'on observe chez d'autres organismes unicellulaires, chez les Infusoires, par exemple, où l'on voit des

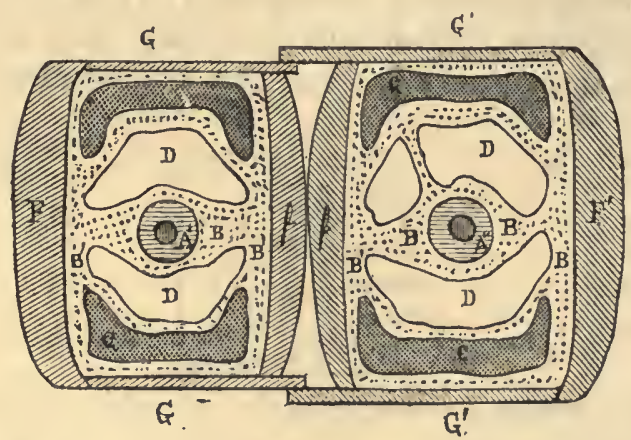

Fig. 16. - Cellule dialomée en voie de division.

f, $\mathrm{f}^{\prime}$, valves nouvelles. - Pour le reste, mêmes lettres que ci-dessus.

organes nouveaux se créer de toutes pièces, des cils se former, des cirrhes émigrer en des points différents de la surface du corps, etc.

Dans tous ces organismes, le phénomène est de même ordre et de même nature, - c'est la division cellulaire, - mais il s'accompagne de circonstances diverses en rapport avec la nature de la cellule qui se divise.

Il arrive souvent que les frustules qui se soni multipliés par division restent adhérents les uns aux autres, comme les cellules des tissus. On obtient ainsi des groupements ou des thalles cellulaires, de formes variées, suivant la forme des frustules eux-mèmes, c'est-à-dire des filaments diversement contournés. Et, si la multiplication se fait plus activement à l'extrémité, les filaments vont en diminuant de diamètre.

C'est en considération de ce phénomène que M. Deby regarde les Diatomées comme des thallophytes; seulement, s'il y a des cas oú le thalle reste agrégé, comme chez les espèces filamenteuses, il y en a d'autres oủ il se désagrège, chacune des cellules se séparant complètement, au moment de la division, de celle qui lui a donné naissance. 
On voit donc, que l'on peut rencontrer chez la mème espèce de Diatomée, selon son élat de développement, des individus possédant :

$1^{\circ}$ Deux valves, un connectif et un noyau. (Etat simple, au repos).

2. Deux valves. deux connectifs superposés et un noyau. (Etat simple et complet, au repos).

$3^{\circ}$ Deux valves, deux connectirs, deux noyaux. (Commencement de la division.

$4^{\circ}$ Quatre valves, deux connectifs, deux noyaux. (Suite de la division).

$5^{\circ}$ Quatre valves, quatre connectils, deux noyaux. (Fin de la division).

Il faut ajouter que souvent le connectif externe est caduc et se détache spontanément (1).

Chez les Diatomées, nous l'avons dit, comme chez tous les organismes qui se multiplient par division fissipare, la taille diminue à mesure que le nombre des divisions augmente, de sorle que l'on pourrait craindre que la dimension des cellules diminuât ainsi indéfiniment. Mais il se produit chez ces Algues, le même phénomène que chez tous les êtres unicellulaires

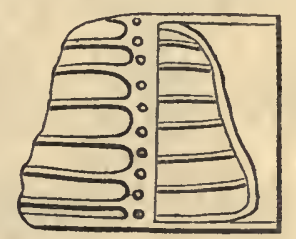

Fig. 17, - Isthmia, frustulc formé de deux valves et un connectit.

voisins : ả la suite d'une série plus ou moins longue de mulliplications asexuelles, par fissiparité, intervient une génération d'ordre sexuel qui rétablit la taille et reconstitue le type de l'espèce.

Autant qu'on en a pu juger par le très petit nombre d'observations qui ont été faites sur la reproduction sexuelle des Diatomées, observations qui, dit M. H. Van Heurck, ne portent que sur 75 espèces appartenant à 30 genres, cette reproduction sexuelle doit se faire assez rarement.

D'ailleurs, ainsi que nous l'avons déjà indiqué plus haut, la décroissance de la taille peut ne pas se faire avec la rapidité que l'on pourrait supposer. Cette constitution de la cellule en deux valves, dont l'une est toujours plus petite que l'autre, semblerait devoir amener une décroissance continue, et pour ainsi dire suivant une progression géométrique, de la dimension des frustules issus de divisions successives. Or, c'est précisément cette disposition qui contribue à retarder considérablement l'amoindrissement de la taille.

On peut se rendre compte plus facilement de ce phénomène en consi-

(1) J. DEur, - Ce que c'est qu'une Diatomée. (Journ. de Micr., 1877). 
dérant une espèce filamenteuse, dans laquelle les frustules, au lieu de se séparer après cháque dédoublement, restent accolés de telle sorte qu'il est possible de les comparer les uns aux autres.

C'est, du reste, ce qu'a fait M. Otto Müller quui a étudié le Melosira arenaria (Moore) et il a tiré de ses recherches une "loi " qui n'est pas facile à comprendre (1). Nous nous bornerons à faire remarquer les faits suivants :

(1) Otto MüLler. Die Zellhaut und das Geset: der Zelltheilungsfolge von Melosira arenaria, Moore. - Berlin, in $-8^{\circ} 1883$, avec $5 \mathrm{pl}$.

Voici l'exposé de la “ loi » de M. O. Müller, tel que le donne le Dr H. van Hourck (Syn. des Diatomées, p. 13).

" Considérés isolement, les individus-frustules (ou individus-cellules, si l'on veut) qui composent un filament de Melosira arenaria, Moore, ont une valeur biologique inégale. Comme expression extérieure de ce fait on re. marque que dans plusieurs individus, le bord d'une ou des deux valves (auquel bord la membrane connective est attachée), est épaissi d'une manière particulière; cet épaississement manque dans les autres individus. La plus jeune valve de chaque frustule reste, jusqu'au commencenenl du moment de la division, dépourvue de membrane connective et est enveloppée par la membrane connective de la valve plus âgée.

La structure de la membrane connective, différente de celle de la membrane de la valve, de même que les délimitations latérales du filament permettent une certaine distinction microscopique entre la valve librc (plus âgée et non recouverte par la membrane connective) et entre la (plus jeunel valve enveloppée par la membrane connective de la valve plus âgée de la même cellule. La succession, la disposition relative et la siructure anatomique men. tionnée des valves libres et des valves enveloppées, conduit à distinguer, dans le filament, des groupes de cellules jumelles et trigémelles disposẻes régulièrement.

On peut démontrer, de la façon la plus rigoureuse, que les cellules dont la valve enveloppée possède l'épaississement du bord sont produites par leurs aïeules spéciales en qualité de cellules filles plus grandes; celles, au contraire, où cet épaississement manque sont produites en qualité de cellules-filles plus petites.

Sitòt que les grandes cellules, et les cellules filles plus petites, peuvent èlre distinguées d'une façon certaine, on peutramener, par l'éliminalion des éléments de dernière formation (des valves enveloppées) les groupes de jumeaux et de trigémeaux à leurs cellules-aïeules de $\mathrm{N}_{1}{ }^{\circ}$ ou $\mathrm{N}_{2}{ }^{\circ}$ période divisionnaire et retrouver leur disposition anatomique spéciale et leur situation relative dans le filament de chaque période.

Si de cette façon on reconstitue l'arbre généalogique du filament, on trouve alors que les plus petites cellules.filles, très reconnaissables morphologiquement par le défaut d'épaississement du bord des valves plus jeunes, franchissent régulièrement une période divisionnaire (génération); elles subissent ainsi un ralentissement de la durée de la division.

Comme résultat de ce qui vient d'ètre dit, oa peut formuler la loi sıivante : " La plus grande cellule-fille se divise, dans la période divisionnaire consécutive, la $\mathrm{N}+1^{a}$ période ; la plus petite cellule-fille, au contraire, se divise d'abord régulièrement dans la $\mathrm{N}+2^{\circ}$ période. $n$

Cette loi occasionne non seulement un retard considérable dans la multiplication par la division, mais met aussi un obs!acle notable à la diminution 
Dans un filament de Diatomées, tontes les cellules n'ont pas la même valeur biologique. Il en est de mème, du reste, chez beaucoup d'Algues confervoïdes. Les unes sont destinées à une multiplication plus prochaine et plus active que les autres. D'après M. 0. Müller, ces cellules peuvent même être distinguées par certains caractẻres morphologiques, l'épaississement du bord des valves, par exemple, (chez le Melosira arenaria, au moins). Or, si nous supposons une Diatomée filamenteuse théorique, nous voyons une de ces cellules (A, fig. 18), produire par une première division une cellule B, dont la plus grande valve $a$ estégale à la petite valve, enveloppée, $b$, de la cellule $\mathrm{A}$. Si les cellules restent accolées, on aura un commencement de filament, 1 , composé de la cellule mère $\mathrm{A}$ et de la cellule fille $B$.

Si la cellule A subit une seconde division, elle produira une seconde cellule $\mathbf{B}^{\prime}$, semblable comme taille à la cellule B. Celle-ci est repoussée par la nouvelle venue qui s'intercale entre elle et la cellule-mère A, pour

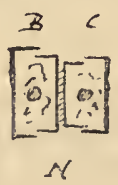

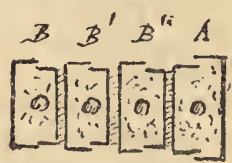

3

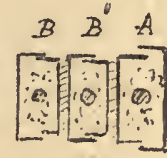

2

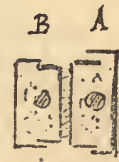

1

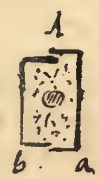

Fig. 18. - Décroissance de la taille par les divisions successives.

former le filament 2, dans lequel la taille est restée la même que dans le filament 1.

La cellule A continuant à proliférer, donne une nouvelle fille B", qui s'intercale entre elle et $\mathbf{B}^{\prime}$, et le filament, 3 , s'allonge encore, mais la taille ne diminue pas.

La cellule aïeule $\mathrm{A}$, qui a le maximum de la taille, peut donner ainsi une longue série de grandes cellules filles $\mathbf{B}, \mathbf{B}^{\prime}, \mathbf{B}^{\prime \prime}, \mathbf{B}^{\prime \prime \prime}$, etc., et le filament s'allongera beaucoup sans diminuer de taille. Une diminution de taille ap-

constante des cellules; il en résulte que la diminution de taille ne se fait pas simultanément avec la multiplication par division et que cette diminution ne se produit que dans un rapport borné.

Pour autant que l'on puisse rapporter la naissance des auxospores à la diminution de taille des frustules, la rareté de la production de ces derniers s'explique par les phénomènes qui viennent d'être décrits.

L'effet de la loi est énergique : si, par exemple, la diminution de taille après 43 divisions est telle, dans les cellules du Melosira arenaria, que la production des auxospores soit nécessaire, comme on peut l'admettre d'après les faits connus, il résulte de la loi énoncée qu'un seul auxospore se produira dans le cas actuel tandis que si la division se faisait d'après la règle généralement admise et qu'énonçait M.Deby, alors il aurait du naître 1 .052.000.000.000 auxospores. " 
paraitra seulement quand la cellule $\mathrm{B}$, première cẹllule-fille de $\mathrm{A}$, (ou une de ses sœurs $\mathrm{B}^{\prime}, \mathrm{B}^{\prime \prime}$, ou $\mathrm{B}^{\mathrm{n}}$ ) va se meltre à se diviser à son touren donnant des cellules $\mathrm{C}, \mathrm{C}^{\prime}$, d'une taille encore diminuée, lesquelles vont s'intercaler dans la continuité de filament, la prolifération ou l'allongement se produisant toujours du côté de la valve enveloppée.

Une seconde diminution de taille apparaitra quand une des cellules de troisième génération $\mathrm{C}, \mathrm{C}^{\prime} \mathrm{C}^{\prime \prime}$ se mettra à se diviser et à former des cellules encore plus petites $\mathrm{D}, \mathrm{D}^{\prime} \mathrm{D}^{\prime \prime}$, etc.

On voit que la continuation de la division dans les grandes cellules, comme A par exemple, entretiendra très longtemps la taille sans diminution. Si, plus tard, la division se produit dans les cellules B, il y aura un amoindrissement, qui persistera tant que les cellules $\mathrm{B}, \mathrm{B}^{\prime}, \mathrm{B}^{\prime \prime}$ se diviseront seules. Nouvelle diminution quand les cellules $\mathrm{C}, \mathrm{C}^{\prime}, \mathrm{C}^{\prime \prime}$, se diviseront, et ainsi de suite, et peu à peu le filament ira en s'amincissant si les divisions se font ainsi régulièrement, les nouvelles cellules s'ordonnant par rapport à leur âge relatif. Et le maintien de la taille à chacun de ces divers degrés pourra se prolonger très longtemps.

Si les divisions ne se font pas régulièrement en procédant des cellules plus anciennes aux plus jeunes, mais qu'elles se produisent simultanément entre des cellules de divers ages, cellules A, B ou C par exemple, il se formera çá et lả dans la continuité du filament des différences brusques de taille, augmentation et diminution, comme cela a lieu dans le Melosira varians.

Quant aux faits que M. Otto Muller a signalés sur l'ordre de division des cellules du Melosira arenaria il est clair qu'ils ne constituent pas une $l o i$, et du reste qu'ils ne se présentent très probablement pas de même chez toutes les éspèces. Il est clair aussi que, chez les espèces non filamenteuses et dont les frustules se séparent aussitôt qu'ils sont formés, des faits du même ordre interviennent pour ralentir le rapetissement de la taille bien qu'on ne puisse pas les observer.

Reproduction par conjugaison. ou rajeunissement. - Quel que soit le ralentissement que le mode de succession des divisions fissipares apporte a la diminution de la taille des frustules, ceux-ci ne finissent pas moins par diminuer de grandeur. 'Toutelois, la diminution n'est pas indéfinie, et il y a pour chaque espéce un minimum de taille qu'elle ne dépasse pas. Il intervient alors un phénomène, évidemment de nature sexuelle, une conjugaison plus ou moins évidente, dont le résultat est la formation d'une sorte de spore, de très grande taille, que M. E. Pfitzer a appelée auxospore el dont sort un frustule qu'on appelle frustule sporangial et qui a repris la taille maxima de l'espèce.

Malheureusement, comme nous l'avons dit, ces phénomènes de reproduction sexuelle des Diatomées nous sont encore très mal connus et, depuis bien des années déjà, la science ne s'est enrichie à ce sujet que d'un très 
petit nombre de faits. Les cas de reproduction sexuelle paraissent relativement rares ou, du moins, " nous ne connaissons, dit M. Paul Pelit, ni l'époque exacte à laquelle le phénomène se produit pour chaque espèce, ni les causes climatériques qui le font naître. Nous sommes donc réduils à attendre que le hasard favorise nos recherches. "

Quoi qu'il en soit, le phénomène peut se produire sur un seul frustule ou sur deux frustules, mais toujours, naturellement, surceux de plus petite taille.

Le premier acte du processus consiste en une sécrétion abondante de la matière mucilagineuse transparente qui entoure le frustule. Celui-ci s'enveloppe ainsi d'une couche épaisse de mucilage; puis, son protoplasma intérieur se dilate se concentre sous forme globuleuse et, sous sa pression, les deux valves se disjoignent le long de la suture des connectifs, s'écartent et se séparent. Le globule protoplasmique, dont le volume est très augmenté, est ainsi mis en liberté dans la masse gélatineuse. ll s'entoure d'une mem. brane à double contour et forme un corps ovoïde dans lequel on constate ordinairement que le protoplasma interne est ld'abord réparti en deux masses plus ou moins distincles qui paraissent fusionner ensuite. Ce corps ovoïde est un sporange.

Bientôt, dans l'intérieur de ce sporange, il se développe un corps de forme variable suivant les genres et rappelant celle du frustule primitif. Ce corps grandit rapidement, s'enveloppe d'une membrane plus ou moins incrustée de silice et souvent plissée en travers. C'est l'auxospore; celle-ci, par sa croissance, fait éclater l'enveloppe du sporange qui se rompt en deux moitiés. Parfois, en grandissant, l'auxospore reste coiffée à ses deux extrémités d'une calotte sphérique, débris de la membrane du sporange.

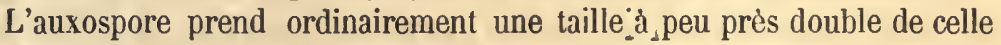
du frustule qui l'a produite; alors, à travers sa membrane transparente, on voit apparaitre dans sonintérieur un nouveau frustule doué de la forme caractéristique de l'espèce et ayant fait retour à la taille maxima de celleci. C'est un frustule sporangial.

Enfin, la membrane de l'auxospore,se brise, le frustule】sporangial est mis en liberté, la masse mucilagineuse au sein de laquelle tous ces phénoménes se sont passés se résorbe, et se réduit en cette couche mince qui subsiste autour de tous les frustules normaux.

Voilå la Diatomée remontée à sa taille et_qui va maintenant se mulliplier par division jusqu'ả la formation d'une nouvelle auxospore.

Que s'est-il passé dans ce phénomène ?

Est-ce une fécondation? - La fécondation résulte, comme on le sait, du mélange de deux plasmas dont l'un joue le role d'élément mâle et l'autre d'élément femelle. Il faudrait donc admeltre, dans ce cas oủ un seul frustule intervient, que le protoplasma de cette cellule s'est préalablement divisé en deux, peut-être en ¡vue d'une fissiparilé qui ne s'est pas opérée, et que ces deux portions se sont polarisées, différenciées, puis, lors de la mise en liberté de la masse plasmatique, se sont fusionnées, jouant l'une par 
rapport à l'autre le rỏle d'élément mâle et d'élément femelle. - C'est ainsi d'ailleurs, que les choses sont ordinairement interprélées.

Est-ce ce qu'on appelle un rajeunissement protoplasmique, phénomène dans lequel le protoplasma subit un remaniement qui le renouvelle, pour ainsi dire, et ravive ses propriétés ? - Il faut avouer, du reste, qu'on ne sait pas du tout en quoi consiste ce rajeunissement, et nous ne voyons pas de raisons probantes pour qu'on ne puisse l'interpréter comme une fécondation d'une partie du protoplasma par l'autre. Quoi qu'il en soil, nous pensons qu'on doit le considérer comme un acte d'ordre sexuel, dans lequel les deux protoplasmas agissant l'un sur l'autre ne sont pas encore séparés ni individualisés; c'est un acheminement vers la fécondation proprement dite opérée par' deux éléments protoplasmiques distincts.

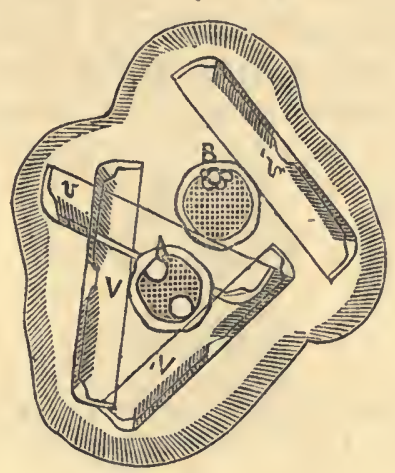

Fig. 19. - Conjugaison de deux Dialomées.

$\mathrm{V}, \mathrm{V}^{\prime}$ valves de premier frustule $; v, v^{\prime}$ valies du second frustule. A, B, masses protoplasmiques des deux frustules mis en liberté par la séparation des valves.

D'autre part, cette production de l'auxospore est elle un phénomène comparable à l'enkystement dont tant de Protozoaires nous donnent des exemples et dans lequel on voit, au sein du kyste, se produire une sorte de fusion de tous les éléments de l'animal ? Le kyste rompu, il en sort des corps reproducteurs ou l'animal lui-même dans de nouvelles conditions.

A toutes ces questions, il n'a pas encore été possible de répondre d'une maniẻre positive.

Le même phénomène de production de l'auxospore peut aboutir à la formation de deux auxospores au lieu d'une. Sauf cette particularité, le processus est absolument le même que celui que nous venons de décrire. Ce mode de reproduction est attribué par IV. Smith aux Achnanthes et aux Rhabdonema. Toutelois, M. H. van Heurck fait remarquer que ce détail n'a été confirmé par aucun auteur plus récent et qu'il pourrait bien y avoir' là une erreur d'observation. 
Eufin, la production des auxospores peut résulter du concours de deux individus de la même espèce (Fig. 19). C'est, pour ainsi dire, le processus normal ; on admet qu'il y a conjugaison entre les deux frustules, comme cela se produit chez beaucoup d'autres Algues, chez les Zygnémées, par exemple, et fécondation de l'une par l'autre, - peut étre fécondation réciproque.

Les choses se passent comme dans le cas précédent, les deux frustules s'appliquent étroitement l'un contre l'autre le long d'une des sulures de leur's valves et sécrètent une grande quantité de matière mucilagineuse transparente. Celle-ci prend souvent une forme déterminée, ovoïde par exemple, et enveloppe exactement les deux frustules conjugués. Bientôt, les valves de ceux-ci s'entrouvent le long de la suture de conjugaison et les deux masses protoplasmiques, qui ont grossi et pris une lorme globuleuse, peurent agir l'une sur l'autre. Du reste, elles sont bientôt mises en liberté dans le thalame gélatineux, par la séparation complète des valves de leurs frustules respectifs, et peuvent fusionner pour donner naissance à un seul globule arrondi qui correspond ả la zygospore des Algues zygnémées. Cette

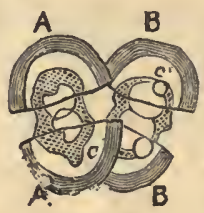

Fig. 20. - Deux sporanges A, B, brisés par le développement intérieur des deux auxospores $\mathrm{C}, \mathrm{C}^{\prime}$. (La substance interne de chaque sporange est divisée en deux masses) (J. Deby).

zygospore peut rester unique ou subir une division en deux segments; c'est un ou deux sporanges qui se recouvrent rapidement d'une membrane relativement épaisse (1).

M. Lüders (2) pense que dans la formation de deux sporanges, il y a double fécondation, c'est à dire qu'il se produit d'abord une différenciation des plasmas à l'intérieur de chacun des frustules conjugués et la partie mâle de chaque frustule se conjuguerait avec la partie femelle de l'autre.

Cette interprétation nous parait très admissible; elle concorde avec l'explication la plus plausible que l'on a donnée de la formation de l'auxospore par un frustule unique dans lequel le protoplasma se différencie et la partie jouant le rôle de mâle se conjugue avec la partie jouant le rôle de feinelle dans le mème frustule. Néanmoins, M. P. Petit la repousse

(1) W. Sult, Synopsis. - Thwaites, (Ann. and Mag. Nat. Hist. T. XX, série I, 1847. - GARTER, Ann. and.Mag. Nat. Hist. T. XVII, série II, 1886. E. Pfitzer, Bau und Entwick. der Diat. Bonn. 1871. - J. Deby, Ce que c'est qu'une Diatomée. (Journ. de Micrographie, 1877).

(2) Luders. - Bot. Zeitung, 1882, nos $7,8,9$. 
parce que, dit-il, si les choses se passaient ainsi les deux auxospores, résultant d'une véritable conjugaison se trouveraient placées perpendiculairement au grand axe des frustules parents, ce qui n'est pas. - C'est lá, à notre avis, une raison par analogie ou par induction, qui ne nous parait pas absolument concluante. Les snasses protoplasmique sont libres dans un milieu mucilagineux demi-liquide et nous pensons qu'elles peuvent s'orienter dans le sens qui leur est fourni par cette enveloppe elle-mème, ordinairement un ovoïde dont le grand axe est parallèle à celui des frustules conjugués.

Quoi qu'il en soit, il se forme un ou deux sporanges, lesquels s'entourent d'une double ou triple enveloppe et dans l'intérieur desquels le plasma paraît bien former, au moins pendant un temps, (et nous sommes surpris qu'on n'en ait pas fait la remarque jusqu'à présent), deux amas distincts conienant quelques gouttelettes graisseuses.

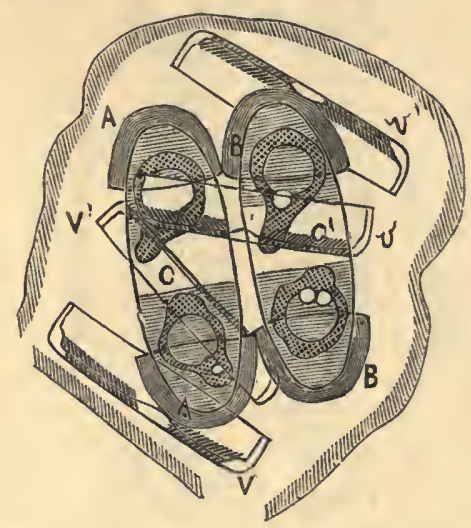

Fig. 21. - Développement des auxospores.

$\mathrm{VV}^{\prime}, v v^{\prime}$, valves vides des deux frustules. - A, B, les deux sporanges rompus, contenant les auxospores $\mathrm{C}, \mathrm{C}^{\prime}$, dont le plasma est nettement divisé en deux masses (J. Deby).

Puis, dans chaque sporange se développe une auxospore dont la croissance fait éclater la membrane du sporange. Et, pendant un certain temps, dans l'auxospore, on reconnait encore la distribution du plasma en deux masses distinctes.

Bientố les auxospores prennent une taille très considérable, double ou triple de celle des frustules primitifs, revêtent une forme spéciale, le plus souvent se strient en travers, se silicifient, et, dans leur intérieur, on voit apparaitre le frustule sporangial.

Tel est le phénomène de la reproduction sexuelle la mieux connue chez les Diatomées et telle est l'interprélation que la plupart des auteurs lıi donnent, dans des différents cas, lesquels peuvent être résumés ainsi :

$1^{\circ}$ Un seul frustule : autofécondation, produisant un sporange et une auxospore. 
2. Un seul frustule : autofécondation, produisant deux sporanges eldeux auxospores (?).

$3^{\circ}$ Deux frustules conjugués, fécondation unique : un sporange, une auxospore.

$4^{\circ}$ Deux frustules conjugués, fécondation simple ou double (croisée) :

Si la fécondation est simple: zygospore unique se dédoublant par fissiparité, d'où deux sporanges et deux auxospores.

Si la fécondation est double : deux zygosporanges et deux auxospores.

M. Paul Petit qui a observé la reproduction du Cocconema cistula refuse á ce phénomène le caractère d'une génération sexuelle. Pour lui, il n'y a que rajeunissement des plasmas, mais non fusion de l'un avec l'autre. Voici la description qu'il en donne.

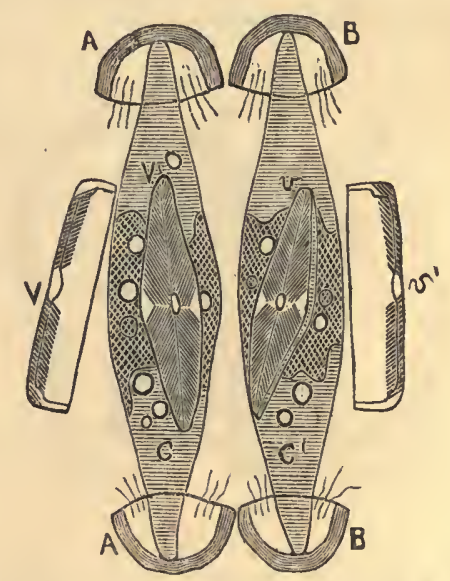

Fig. 22. - Auxospores mûres (d'après M. J. Deby). Mêmes lettres que ci-dessus.

"Chez le Cocconema cistula (PI. I, fig. 1), comme chez toutes les Cymbellées, le chromatophore, sous forme d'une seule lame, recouvre les deux valves et l'un des côtés de la zone d'emboitement, comme le montre le schéma (fig. 13) qui représente une coupe perpendiculaire au grand axe du frustule et passant par le centre. Lorsque le moment de la reproduction est arrivé, deux frustules se rapprochent et se mellent en opposition par leur côté le moins cintré. ll se produit, aussitôt après, une abondante sécrétion d'une matière gélatineuse, incolore et transparente, qui forme autour des deux frustules une large enveloppe ovoïde, complètement fermée, cette matière gélatineuse pourrait peut-être bien provenir de l'accroissement fort grand de l'enveloppe gélatineuse qui entoure d'ordinaire toutes les Diatomées. "

- Dès que l'enveloppe gélatineuse a pris tout son accroissement, on voit, dans chacun des frustules, les chromatophores s'agglomérer vers le 



\section{PLANGHE I}

Figure 1. - Frustule du Cocconema Cistula, Ehb.

Figure 2. - Frustules réunis et entourés d'une masse gélatineuse, avec le plasma aggloméré aú centre.

Figure 3. - Frustule vu de côté, laissant voir les valves écartées par la dilatation du plasma.

Figure 4. - Sortie du plasma pour constituer les auxospores.

Figures 5, 6, 7, 8. - Divers états de développement des aurospores.

Figure 9. - Les chromatophores prennent leur forme naturelle; les auxospores deviennent arquées.

Figure 10. - Auxospores fortement arquées.

Figure 11. - Auxospores avec membrane plissée.

Figura 12. - Auxospore (fortement grossie, 800 diamètres) laissant voir le plissement de la membrane et les commeucements de la ligne médiane.

Figure 13. - Coupe d'un frustule perpendiculairement an grand axe (schéma).

Figure 14. - Navicula crassinervia (de Bréb.) avec deux auxospores, vu de face.

Figure 15. - Navicula crássinervia (de Bréb.) avec auxospore plissée (vu de côté); la deuxième auxospore est masquée.

(D'après M. Paul Petit, Bull. de la Soc. Bot. de France,

T. XXXII, 1885.) 


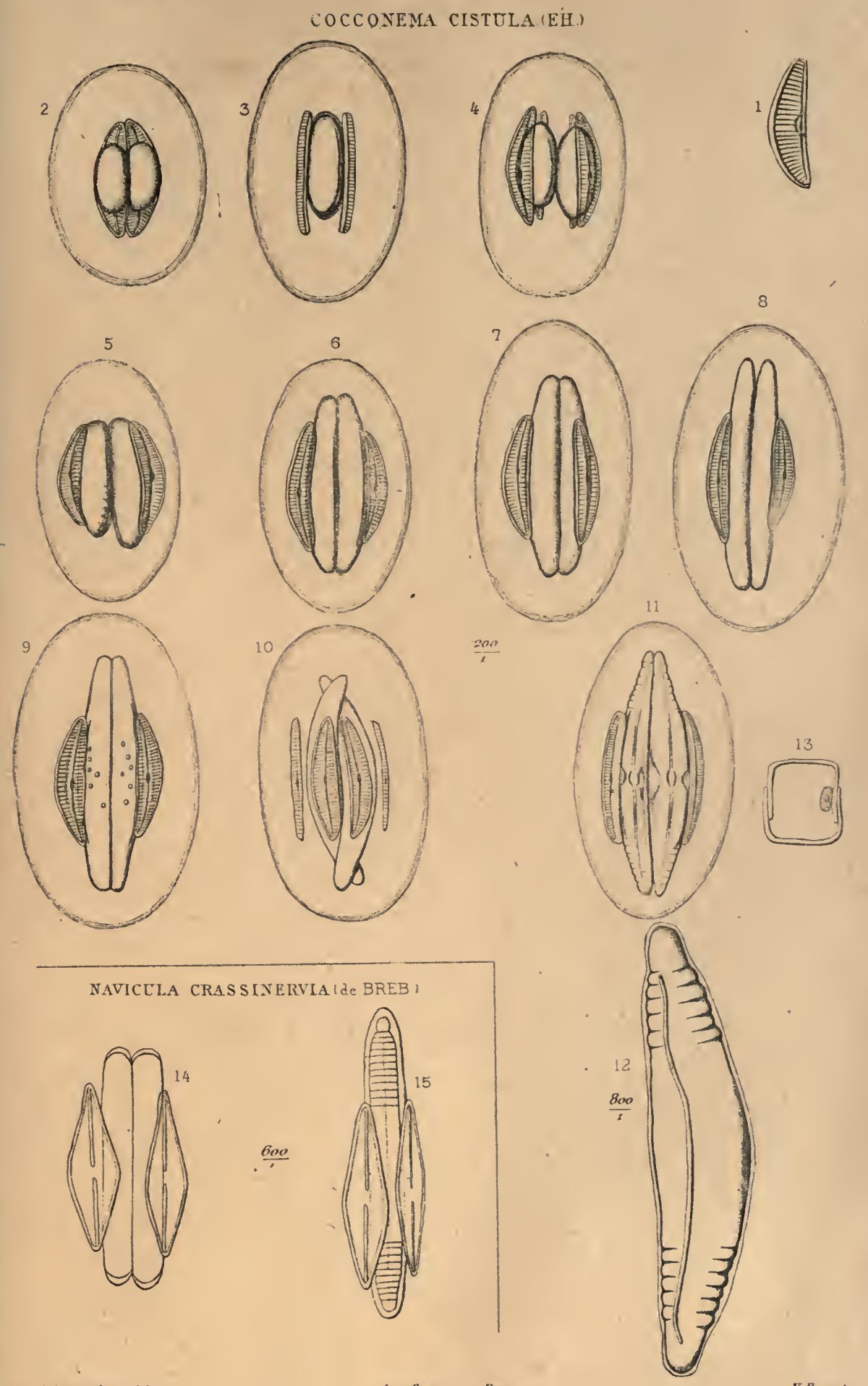



centre (Pl. I, fig. 2) en se retirant des extrémités. Bientỏt le plasma concentré, au milieu du frustule, sous forme d'une masse ellipsoïde, augmente de volume et oblige les deux valves du frustule à s'écarter (Pl. l, fig. 3) ; ce que l'on constate facilement en faisant rouler les frustules dans la préparation. Les masses protoplasmiques sortent alors des frustules par les cólés les moins cintrés, qui se sont entr'ouverts, et viennent se placer l'une contre l'autre et parallélement aux valves des deux frustules vides (Pl. I, fig. 4), sans qu'il y ait jamais de fusion entre les deux masses. Ce fait enlève donc toute idée de conjugaison; c'est un simple rajeunissement de la cellule, une reproduction asexuée. "

- Les deux masses protoplasmiques constituent les auxospores; elles renferment les noyaux ; elles sont nues et ont une teinte brun foncé dans toute leur étendue (Pl. I, fig. '5). Le volume des auxospores s'accroit rapidement, surtout dans le sens de la longueur (fig. 6 et 7); il atteint le double de la longueur des cellulules mères (fig. 8). "

"Dès que l'accroissement a pris fin, on voit apparaitre une membrane sur les auxospores; le plasma coloré se retire légèrement des extrémités (PI. I, fig. 9) et, bientôt après, ce dernier prend la forme caractéristique du chromatophore des Cymbellées. Aussitôt que le plasma coloré a acquis sa forme définitive, on voit les auxospores, d'abord subcylindriques etdroites, s'arquer plus ou moins fortement (fig. 9 et 10). En même temps, les extrémilés s'atténuent et la membrane ne tarde pas à se plisser vers les deux pointes du frustule, tout en restant lisse au centre (fig. 11), et l'on voit alors apparaître les premières traces de la ligne médiane (Pl. I. fig. 12), A ce moment, l'enveloppe du jeune frustule est plus fortement marquée, c'est le commencement de la silicification, c'est-à-dire du dépôt de la silice dans la cellulose de la membrane primilive. (1). ,

Lả s'arrêtent les observations de M. P. Petit. Il lui a été impossible de suivre plus loin le développement du frustule qui alors se trouve étre deux fois plus grand que la cellule mère.

Le même observateur a aussi rencontré, mais dans un état avancé, les auxospores du Navicula crassinervia. Elles élaient plus ou moins développées. Les unes (PI. I, fig. 14) commençaient seulement à s'accroitre; d'autres (fig. 15̄) laissaient voir, sur les extrémilés, un plissement analogue à celui des auxospores du Cocconema cistula (1).

Ces observatious confirment celles du $\mathrm{D}^{\mathrm{r}}$ Schmitz sur la formation des auxospores du Cocconema cistula (2) et sont, au contraire, en contradiction avec les théories émises par MM. Thwailes, Carter, W. Sinith et Lüders qui croient à une véritable conjugaison sexuelle.

Pour nous, nous croyons à une action sexuelle. Nous voyons qu'il y a

(1) Bull. Soc. Bot. de France, T. XXXII, 1885, (Sess. de Charleville).

(2) Bot. Zeilung, 1872, p. 217. - LF ScHuтz. - Ueber die A uxosporenbildung der Diat., Halle, 1877. 
nécessité d'un contact entre deux protoplasmas, nous pensons que ce contacl a un but, que ce but est une influence qu'ils doivent exercer l'un sur l'autre; nous savons que les observateurs, dans les cas cités plus haut, n'ont pas pu reconnaitre par quel mode, quel mécanisme s'exerce cette influence, s'il y a fusion partielle des proloplasmas, échange d'un élément des cellules pendant la conjonction de ces cellules, - mais quels que soient ce mode et ce mécanisme, de quelque manière que se produise cette influence, nous pensons qu'il y a dans ce phénomène une action sexuelle. Nous rappellerons, à cette occasion, que pendant plus d'un siècle, on a vu les Infusoires s'accoupler, sans pouvoir se rendre comple de la nature du phénomẻne, et c'est seulement à une époque tout-å-fait récente qu'on a reconnu que cette conjugaison s'accompagne de l'échange d'un élément cellulaire, d'un noyau accessoire ou nucléole. Nous pensons qu'il en sera de mème pour la conjugaison des Diatomées et que quand les observations seront plus nombreuses, qu'on aura pu appliquer à cette étude les ressources de la technique moderne, on reconnaîtra dans ce phénomène quelque chose d'analogue a ce qu'on appelle " génération sexuelle " chez les autres Protorganismes. Cette génération, d'ailleurs, n'a pas toujours pour but la production d'œufs, de spores ou de germes, mais d'individus nouveaux, rajeunis si l'on veut, remontés au type de l'espèce, doués d'une activité vitale plus intense et d'une aptitude plus grande à la multiplication fissipare.

C'est là, du reste, le seul phénomène connu qui, dans le cycle biologique d'une Diatomée, rappelle une reproduction sexuelle. D'autres, il est vrai, ont été signalés, mais outre qu'ils sont encore moins étudiés que ceux dont nous venons de parler, ils ne présentent pas une ressemblance aussi frappante avec les processus de génération sexuelle que l'on connaît chez les autres Protorganismes et l'on peut aussi bien les considérer comme une sporulation asexuelle que comme une reproduction sexuelle.

"L'apparition subite d'espèces, dit M. J. Deby, là où précédemment il n'en existait pas; leur succession périodique chaque année en des saisons indéterminées, sans qu'on puisse en trouver dans l'intervalle dans la mème localité, font pressentir la possibilité d'un mode de génération qui n'est pas encore suspecté, par germes, par micro ou macrozoospores, peut-êlre même dans le premier cas avec formation de zygozoospores comme cela a lieu pour tant d'Algues inférieures vivant dans les mêmes conditions que les Diatomées."

En effet, le Dr Matteo Lanzi, lor's de ses études sur la matière mucilagineuse qui entoure le frustule des Diatomées, matière qu'il a, comme nous l'avons dit, regardé comme un thalle, et que nous avons désigné sous le nom de thalame, le $\mathrm{D}^{\mathrm{r}}$ Matteo Lanzi, disons nous, a signalé des faits que nous ne pouvons passer sous silence (1).

M. Lanzr. - Le thalle des Diatomécs (Ann. Soc. B. de Microscopie et Journal de Nicrographie, 1878). 
Pour cet observateur, la couche externe mucilagineuse, qui prend une si grande extension au moment de la "conjugaison, " n'est autre clıse que le protoplasma cellulaire qui a acquis un développement considérable et qui, forçant les valves, non extensibles, à s'entrouvrir, est sorti du frustule. Mais, dès avant celte efiraction, le protoplasma interne avait déjá subi des modifications : il s'y était formé des corpuscules de divers aspects. Les uns sont hyalins sans membrane et ce sont ceux-lả qui, en grossissant, procurent la multiplication du thalame mucilagineux, amorphe; les autres se revêtent d'une large membrane et, après leur sortie, s'organiseront en thalame à forme définie, pédicelles, tubes, etc., si l'espéce en comporte ; d'autres, enfin, sont colorés par de l'endochrôme et toujours en nombre plus grand que deux. Ceux-ci s'enveloppent aussi d'une membrane, s'organisent en cellules, grossissent et deviennent de nouveaux frustules. Ce sont des germes.

Ainsi, lorsqu'un frustule est mùr, son protoplasma interne se différencie, augmente de volume, disjoint les valves, sort, foisonne, s'étale en lames, se gonfle en masse plus ou moins épaisse, se dispose en pédicelles ou en tubes, et entraine les germes qui vont ainsi se trouver répartis @̧a et là dans les diverses expansions du thalame. "Une fois que la masse plasmatique, ainsi organisée, est sortie du frustule, dit M. Lanzi, elle commençe à vivre d'une vie propre, cherche un appui sur d'autres corps, et s'accroît avec une rapidité surprenante. Les germes nouveaux prennent alors la forme des frustules adliérents agrégés ou libres qui leur ont donné naissance."

- Dans une récolte que je fis å Rome, dit le même auteur, je reconnus que quelques morceaux de cette couche, qui faisait adhérer aux parois d'une fontaine un Epithemia ventricosa, étaient formés par une très grande quantité de corpuscules arrondis, de couleur vert-jaunâtre, granuleux. Ces corpuscules étaient en tout semblables à ceux encore renfermés dans d'autres frustules murs d'Epithemia immergés aussi dans leur plasma hyalin, que ces frustules fussent adhérents ou libres. Telle était la ressemblance, qu'on ne pouvait douter que la substance plasmatique du " thalle " entier et les germes qu'il renfermait n'eussent été auparavant réunis dans d'autres frustules semblables à ceux qui en contenaient encore. "(Pl. II).

Dans une autre récolte, de Cymbella (Cocconema) cistula, à l'état de reproduction, M. Lanzi a vu des corpuscules arrondis, très petits, et de la couleur de l'endochrôme. lls étaient contenus dans la couche hyaline mucilagineuse et ressemblaient à d'autres contenus encore dans les frustules. En suivant leur développement, il les a vu croître avec leur forme arrondie, puis s'allonger et prendre la forme lunulée et naviculaire propre aux plus petits frustules. Enfin, ils atteignaient la taille normale, les uns restant adhérents au thalame, les autres devenant libres. Leur nombre était extrèmement considérable, leur taille présentant toutes les dimensions 
entre les deux extrêmes, et l'on ne pouvait admeltre qu'ils résultasseìt d'une fissiparité.

D'autres faits semblables ont été observés par M. M. Lanzi sur des Navicula ambigua, Nilzschia minulissima, Amphora ovalis, et il a suivi, sur le Gomphonema olivaceum, toute la série des transformations depuis l'état de germe contenu dans les frustules mûrs jusqu'à l'état de frustule nouveau parfait. Il a observé en même temps le déreloppement du thalame constituant d'abord une masse amorphe, puis poussant des pédicelles qui se dichotomisaient. De sorte que "quand le cycle végélatif fut complet, la Diatomée montrait trois formes différentes : la forme sessile ou sphénelloïde, la forme pédonculée simple ou dicholome et la forme libre. »

Nous ne reviendrons pas ici sur ce que nous avons dit, et assez longuement, à propos de la nature que M. Matteo Lanzi altribue au plasma extérieur, thalle ou thalame des Diatomées, et sur le rôle qu'il lui prête dans la reproduction ou la mulliplication de ces organismes, mais nous ne pouvons nous empécher de faire remarquer l'analogie évidente qui existe entre tous ces faits, et ceux que nous avons déjả brièvement signalés à propos de la multiplication de divers Sporozoaires, notamment les Psorospermies. Celte comparaison répugne, nous le savons, à la majorité des diatomistes, mais il n'en est pas moins évident que les phénomènes de division, de conjugaison ou de sporulation dont les Diatomées sont le théàtre ont leurs analogues dans la division, la conjugaison ou la sporulation d'autres organismes que leur nature unicellulaire et certaines différenciations spéciales de leurs éléments rendent comparables aux Dialomées. Il n'en est pas moins vrai que l'on peut dire que ces phénomènes ne diffèrent chez les uns et chez les autres que par des détails en rapport avec la structure particulière, les conditions d'existence et la situation de ces divers organismes dans la série des êtres.

\section{\$ 4. - MODE DE VÉGÉTATION DES DIATOMÉES}

On a déjả vu que la cellule Diatomée, qu'on appelle particulièrement frustule, est composée d'une membrane cellulaire formée de cellulose plus ou moins fortement imprégnée de. silice, (comme chez d'autres organismes elle est imprrégnée de carbonate de chaux ou d'autres substances) et d'un contenu.

Ce contenu est formé de protoplasma plus ou moins finement granuleux, renfermant un noyau ordinairement suspendu au milieu de la masse protoplasmique et contenant lui-même un nucléole. $\mathbf{A}$ la surface du protoplasma est un endochrôme disposé de manière variable dans les diverses espèces, mais de manière constante dans la même espèce, formant des chromatophores en plaques, lames ou grains, et pénétrés de cette modification 



\section{PLANCHE II .}

Fıgure 1. - Epithemia ventricosa, Kütz. Thalle contenant des frustules mûrs et des germes (spores) en tout semblables à ceux renfermés dans d'autres frustules libres, comme on en voit dans la figure suivante.

Figure 2. - Frustules libres du mème Epithemia qui contiennent plasma et germes de formation nouvelle.

Figure 3. - Cymbella (Cocconema) cistula, Ehb. Le thalle contient des germes (spores) et des frustules à différents degrés de développement.

Figure 4. - Frustule du même, libre et mûr.

Figure 5. - Gomphonema olivàceum, Kütz. Frustules sporangiaux accrus de volume par la dilatation du plasma qui presseleurs valves. On en voit deux autres en $a$ de la Figure 6.

Figure 6. - Thalle contenant des germes de grandeurs différentes et proportionnelles aux divers degrés de végétation. De plus, on voit : en $a$ deux fustules sporangiaux on croissance; en $b$, la forme sphénelloïde du même Gomphonema; en $c$, sa forme pédonculée.

(D'après le Dr Matteo Lanzl : Le thalle des Diatomées, Journ. de Micr. 1878.) 


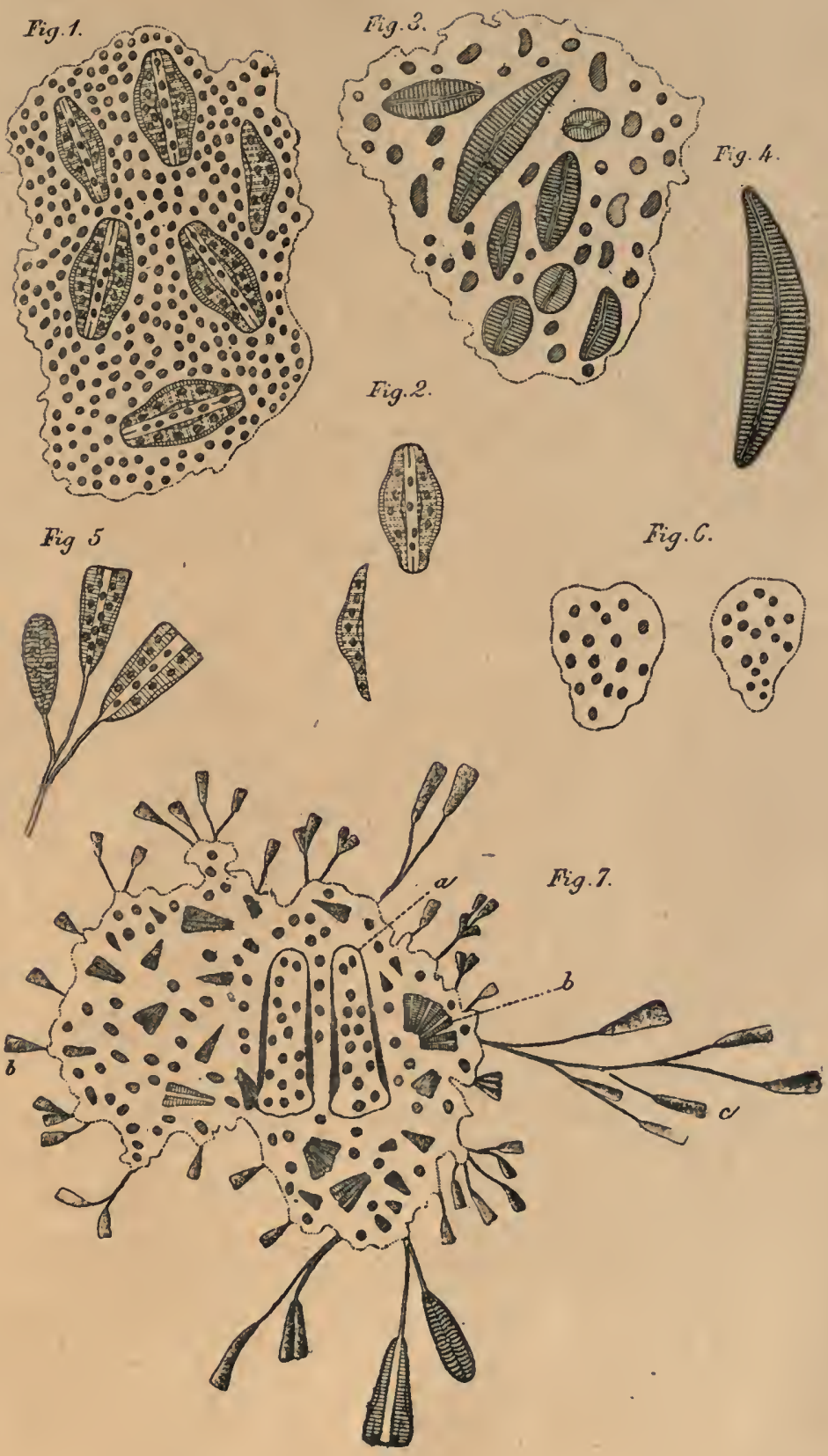



brune de la chlorophylle que l'on a appelée diatomine et dont nous avons indiqué plus haut la composition. Enfin, çà et là sont des gouttelettes huileuses. (Voir Fig. 14).

Au point de vue histologique, le frustule des Diatomées correspond donc à une cellule.

De plus, celte cellule est entourée par une matière mucilagineuse transparente qu'on a appelée coléoderme, thalle, thalame, qui reste souvent à l'élat de mince couche enveloppante, se développe quelquefois en larges masses amorphes ou ovoïdes, ou bien prend la forme de pédicelles, ou stipes, simples ou ramifiés, a l'extrémité desquels sont portés les frustules (Fig. 23); ou bien encore constitue des tubes dans l'intérieur desquels sont contenus les frustules.

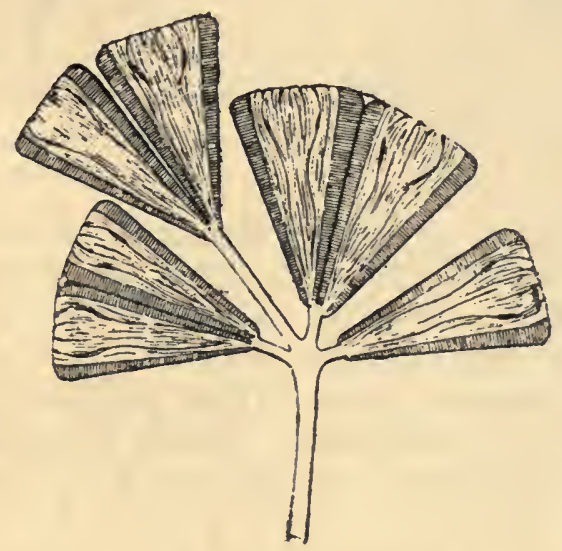

Fig. 23. - Licmophora paradoxa, $\mathrm{Ag}(1)$.

Dans certaines espéces, aprẻs leur formation par fissiparité, les frustules ou cellules restent accolées les unes aux autres, valve contre valve, par cette matière mucilagineuse incolore qui joue alors le rôle de substance intercellulaire. Ces espéces, dites filamenteuses, constiluent ainsi des filaments pluri-cellulaires, de formes diverses, qui sont composés de la même manière que les filaments des 0scillaires, des Spirogyreset d'autres Algues analogues à thalle filamenteux.

On peut supposer même que chez les espèces où l'on trouve les cellules libres et isolées, cet état est produit par la désagrégation continue, et au fur et á mesure de la multiplication, de ces cellules qui ne forment plus, pour ainsi dire, qu'un thalle virtuel. - Sous quelle influence s'est produit, dansl'évolution de l'espéce, cet émiettement ou cette segmentation continuelle

(1) D'après M. A. Truan, Diat. de Asturias. 
du thalle? C'est ce qu'on ignore, mais on peut y voir l'effet des besoins de la dissémination d'une espèce qui se reproduit rarement par génération sexuelle et en donnant seulement un très petit nombre de spores, ou de l'adaptation à des circonstances particulières de milieu.

Quant aux espèces dont les frustules restent accolés, valve contre valve, après la fissiparité et forment des filaments, on comprend que c'est la valve t

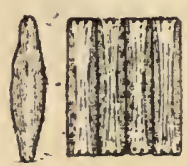

1

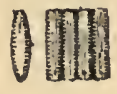

2

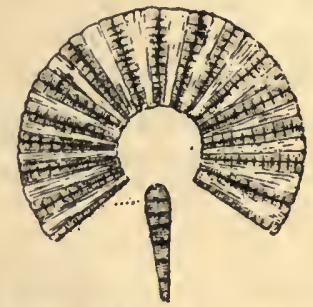

Fig. 24. - 1. Fragilaria virescens. 2. F. mutabilis.

Fig. 25. - Meridion circulaire, Ag.

qui détermine la section ou coupe du filament. Si les valves sont étroites et longues, parallèles l'une à l'autre, le filament sera plus ou moins plat et rubané (Fragilaria); si les valves sont circulaires ou à peu près, le filament sera plus ou moins cylindrique (Melosira).

Mais si les valves ne sont pas sur des plans parallèles, si elles sont inclinées l'une sur l'autre, comme dans les frustules en coin, les filaments ne sont pas droits, mais courbes, tournent en cercle ou même peuvent faire

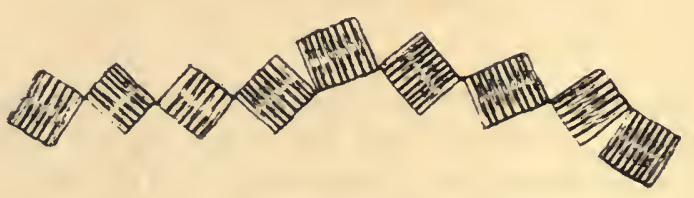

Fig. 26. - Tabellaria flocculosa.

plusieurs tours de spire plus ou moins plats, qui se superposent (Meridion).

Enfin, entre les espèces dont les frustules restent réunis en filaments droits ou courbes, et celles dont les frustules se séparent aussitôt après la fissiparité, il en est dont les cellules restent unies, mais seulement par un point de leur surface, un de leurs angles, par exemple, formant ainsi, dans ce cas, des filaments ou chaines en zigzag (Tabellaria). 


\section{\$ 5. - RÉVIVISCENCE DES DIATOMÉES}

Pendant les chaleurs de l'été, la plupart des fossés et des mares qui contenaient des Diatomées se dessèchent et restent quelquefois pendant de longs mois dans un état de dessication complet; certaines de ces Algues vivent dans la mousse des arbres et des murailles, exposées aussi à de fréquentes périodes de dessication, et cependant, aussitôt que revient l'humidité, aussitôt que les pluies ont rendu de l'eau aux fossés, de la fraîcheur aux mousses et ả l'écorce des arbres, on y retrouve les Diatomées vivantes comme auparavant.

Il était naturel, dès lors, de se demander si la dessication tue définitivement les Diatomées. On n'a jamais constaté, en effet, qu'elles aient la propriété de s'enkyster, comme le font les Infusoires et d'autres protorganismes, ce qui permel à ceux-ci de résister, dans leur kyste, à toutes les influences climatériques. L'enkystement, il est vrai, est une propriété qui ne paraît guère appartenir qu'à des organismes doués de caractẻres plus ou moins manifestes d'animalité, mais il est remplacé chez les végélaux inférieurs par la formation de spores dormantes, dans la coque desquelles le protoplasma végétal conserve sa vitalité, à l'abri des intempéries, comme l'Infusoire dans son kyste.

Or, on n'a pas constaté la formation de ces spores dormantes chez les Diatomées. Il était donc possible que les Diatomées eussent, comme certains animalcules microscopiques, les Rotifères, les Tardigrades, diverses Anguillules, la propriété de résister à une dessication complète, c'est à dire de pouvoir récupérer, après avoir ẻté complètement desséchées, l'eau qu'elles ont perdue et de renaitre à la vie.

C'est ce que M. P. Petit a résolu d'éclaircir par quelques expériences positives (1). Il a laissé sécher dans des vases de terre, á l'abri de la poussiẻre, pendant 6 et 8 mois, des Diatomées vivantes avec leurs substratum vaseux.

Examinant alors les frustules, il a vu que beaucoup étaient vides et par par conséquent morts, mais d'autres avaient conservé dans leur intérieur, à l'une de leurs extrémilés, quelques granulations brunes, restes de l'endochrôme desséché.

Il a alors ajouté dans les vases de l'eau distillée, filtrée et aérée, et les a exposés à la lumière directe du soleil. Il a vu que, dès le quatrième jour, les granulations brunes avaient grossi et repris leur couleur jaune na-

(1) P. Petit. - La dessication fait elle jérir les Diatomées. (Journal de Micrographie, 1877, p.242). 
turelle. Le lendemain, le plasma avait repris environ la moitié de so: volume. Le huitième jour, les frustules avaient recouvré leur aspect ordinaire et l'endochrôme sa disposition caractéristique pour chaque espèce. Les Navicula avaient retrouvé leurs curieux mouvements et bientôt commençaient à se multiplier par division, comme si rien d'anormal ne leur était arrivé.

D'après les expériences de M. P. Petit, pour que la réviviscence puisse se produire, il faut que la dessication ne soit pas trop brusque. C'est, d'ailleurs, ce qui arrive aussi pour les Rotifêres et autres animalcules réviviscents. Ainsi, les Diatomées qui se dessèchent lentement dans la vase retrouvent leur vitalité, tandis que celles qui sont à découvert sont ordinairement saisies par une dessication trop rapide et se désorganisent complètement.

C'est cette dessication lente qui se produit dans la vase des fossés et dans les mares. Il n'est donc pas étonnant que, pendant la saison humide, on voie apparaître, presque subitement, des Diatomées, là oủ on en aurait vainement cherché pendant la sécheresse.

Du reste, sans faire les expériences démonstratives qu'a réalisées M. Paul Petit, nous avions remarqué déjà, qu'après avoir laissé longtemps sèches, faute de penser à y ajouter de l'eau, des Diatomées que nous conservions dans des verres sur notre fenêtre, nous les trouvions toujours vivantes toutes les fois que nous leur rendions l'eau nécessaire.

D'autre part, M. F. Habirshaw, en Amérique, avait constaté le mème fait en 1871. Il était connu depuis assez longtemps par plusieurs diatomistes, notamment par M. J. Deby. - Néanmoins, il n’avait pas été constaté scientifiquement avant les expériences de M. P. Petit (1).

Ajoutons que malgré les précautions qu'on peut prendre, comme une dessication pas trop brusque, pour obtenir la réviviscence des Diatomées, toutes les espéces, et même tous les individus d'une même espèce, sont loin de se prêter avec la même facilité à cette expérience. - Il en est de même, d'ailleurs, pour les Rotifères et les autres animalcules qui présentent cette propriété.

(1) J. Pelletax. - La Réviviscence des Diatomées. (Journ. de Micrographie, 1878, p. 124). 


\section{STRUGTURE MIGROSCOPIQUE DES DIATOMÉES}

Nous avons étudié d'une manière générale la constitution des Diatomées, il nous reste à examiner les divers éléments qui composent leur frustule.

\section{\$1. - VALVES.}

Nous savons déjá que le frustule est toujours composé de deux valves réunies l'une à l'autre le long de leur ligne de suture par une membrane

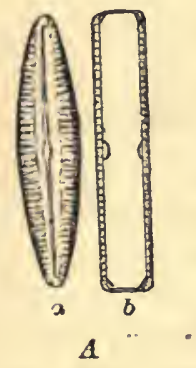

1

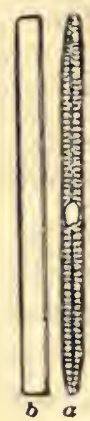

$B$

2

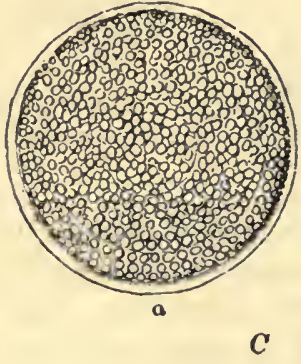

3

C

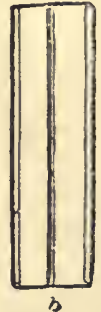

Fig. 27.

1. - Pinnularia viritis vu de face et de profil. - a, Vue de profil, ou face valvaire (side view). - $b$, Vue de face, ou face connective (front view).

2. - Synedra ulna. - a, Vue de profil, face valvaire $(s . v$,$) . -b$, Vue de face, face connective $(f \cdot v)$.

3. - Coscinodiscus patula. - a, Vue de profil, face valvaire, (s.v.). - b, Vue de face, face connective, $(f \cdot v \cdot)$.

formant comme un anneau ou un ruban et qu'on appelle zone connective ou suturale ou simplement connectif.

Nous savons de plus qu'à l'état de développement complet, le connectif est formé de deux membranes qui se recouvrent plus ou moins l'une l'autre, l'une adhérant aux bords d'une valve, l'autre aux bords de l'autre valve. 
De sorte que le frustule est constitué comıne une véritable boîte, dont les valves forment le fond et le couvercle, et les connectifs les côtés, qui s'emboitent, entrant l'un dans l'autre. Il en résulte que, dans tout frustule, une des valves est toujours sensiblement plus petite que l'autre. Nous avons vu, d'ailleurs, que cette disposition est le résultat du mode de formation des valves lors dela multiplication des cellules par fissiparité.

En raison de cette structure bivalvaire, les fruslules (1), lorsqu'on les examine sur un plan, peuvent se présenter de deux manières : ou bien ils sont, pour ainsi dire, couchés sur le flanc et l'on ne voit qu'une des valves, l'autre étant cachée par dessous. La membrane connective n'est visible qu'en projection sur les bords. C'est ce que la plupart des diatomistes désignent sous le nom de vue de côté ou de profil, ou jace valvaire, et ce que les Anglais appellent side-view. (Fig. 27, a). Ou bien le frustule est, pour ainsi dire, couché sur le dos et l'on voit au milieu la bande connective et de chaque côté, le bord plus ou moins large des deux valves.

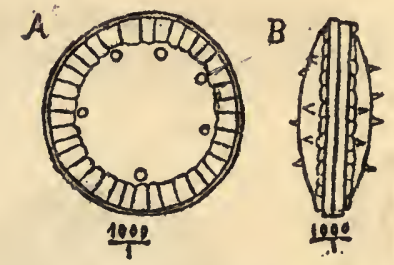

Fig. 28. - Cyclotella sexpunctata. Deb.

A. Face valvaire (side-view). - B. Face connective (front-view).

C'est la vue de face ou face connective, ou face suturale, le front-view des Anglais: Nous conserverons ces désignations consacrées par un usage presque général.

Il faut remarquer que dans certaines espèces, notamment les espèces discoïdes ou polygonales, les valves sont extrêmement larges, tandis que les connectifs sont relativement très étroits. De sorte que le profil, qui montre la face valvaire est très large, tandis que la vue de face ou de front est beaucoup plus étroite. (Fig. 28).

Ces valves ont, du reste, des formes très variées, souvent elles sont beaucoup plus longues que larges avec les longs côtés sensiblement parallèles. (Fig. 27, B). Le frustule a l'aspect d'un bâtonnet (Synedra ulna); on dit que sa forme est bacillaire. C'est de la fréquence de cette forme chez

(1) Remarquons que l'on désigne plus particulièrement sous le nom de frustule l'ensemble de la boite siliceuse d'une Diatomée, indépendamment des protoplasma, noyau, endochrôme. Quand on a détruit par le feu ou les acides toute la matière organique vivante d'une cellule de Diatomée, ce n'est plus une cellule, mais c'est encore un frustule. 
les Diatomées, ou de formes très allongées, que ces Algues avaient reçu jadis le nom de Bacillatiées. Dans un très grand nombre d'espèces les valves sont plus longues que larges, mais en ovale ou en navette ; on dit alors que le frustule est naviculaire (Fig. 29 et 30).

Puis, les valves, et par suite le frustule, peuvent être circulaires, ovales

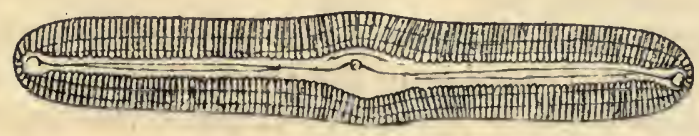

Fig. 29. - Navicula major Kz.

elliptiques, réniformes, cunéiformes, triangulaires, quadrangulaires, etc., désignations qui se comprennent toutes d'elles-mêmes et que nous n'avons pas besoin d'expliquer.

Elles peuvent encore, à leurs extrémités, se terminer par une pointe (acuminées), par une courbe courte (obtuses), par un prolongement rétréci et mousse (rostrées), par un prolongement dilaté en bouton'(capitées), etc.

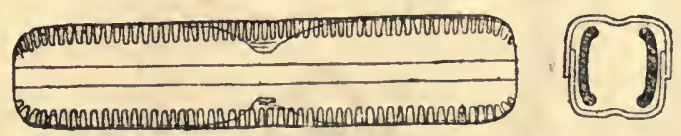

Fig. 30. - Navicula major (face connective et coupe transversale).

Mais, outre les différences de formes, elles peuvent présenter à leur surface des détails importants.

Ainsi, dans un grand nombre d'espẻces, particulièrement celles à forme plus ou moins naviculaire, les valves sont divisées suivant leur grand axe par une ligne trẻs visible et saillante qui les divise en deux moitiés longitu-

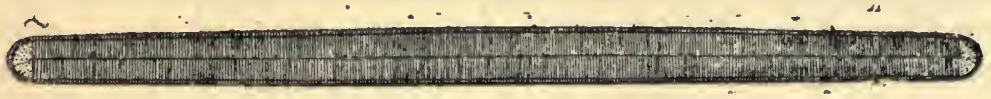

Fig. 31. - Synedra fulgens.

dinales, ordinairement (mais pas toujours) symétriques. Cette ligne s'appelle le raphé ou le rachis (Fig. 29).

Elle se termine ordinairement à chacune de ses deux extrémités prés d'un épaisissement à peu près circulaire qu'on appelle nodule. De même, 
elle est interrompue au milieu par un autre nodule. De sorte qu'il y a deux nodules terminaux et un nodule central ou médian. (Fig. 29).

Toutes les formes allongées ne présentent pas de raphé. Beaucoup, et particulièrement des formes bacillaires, au lieu de cette ligne longitudinale saillante présentent un espace lisse sur lequel cessent les autres détails, côtes ou stries, (qui ornent souvent les deux côtés de la valve. C'est un pseudo-raphé (Fig. 31.)

D'autres enfin, comme les formes larges, circulaires ou polygonales, ne présentent ni raphé ni pseudo-raphé médian. (Fig. 28).

C'est précisément sur la présence ou l'absence d'un raphé ou d'un pseudo-raphé que M. H. L. Smith a fondé sa classification, si connue, des Diatomées, classification dont nous aurons à nous occuper.

Qu'est-ce que le raphé ? Que sont les nodules?

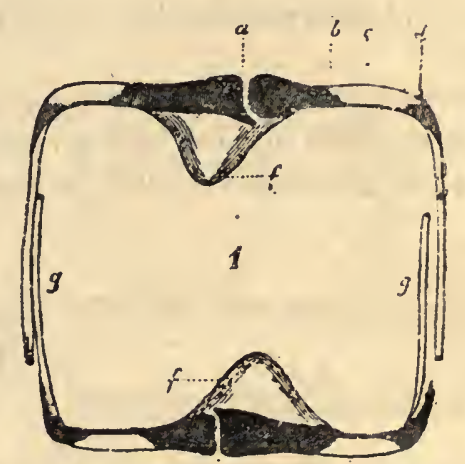

Fig. 32. - Coupe d'un grand Navicula (1500 diam.)

$a$, raphé ; $b, c, d$, un des côtés de la valve; $f, f$, nodules médians ; $g$, connectifs. (D'après M. W. Prinz).

Le raphé est évidemment une arèté saillante, épaissie, faisant saillie ordinairement à la face extérieure de la valve et à sa face interne; mais cette arête saillante est sillonnée par une véritable fente par laquelle le contenu de la cellule se met en rapport avec le monde extérieur. Strasburger admet même, ce qui nous parait au contraire trẻs peu probable, que le protoplasma interne śmet par cette fente une bande mince, une sorte de pseudopode, qui serait l'organe du mouvement de la Diatomée.

Quoi qu'il en soit, il paraît certain que le raphé est une fente longeant ou sillonnant un épaississement plus ou moins considérable de la val ve. Ce fait est mis en évidence, d'ailleurs, par les coupes que l'on a pu obtenir accidentellement de frustules à raphé. Telle est celle que représente la fig. 32, trouvée par M. W. Prinz, sur un grand Navicula du dépôt de Franzensbad.

Le même fait peut aușsi ètre çonstaté de plano sur d'autres frustules 
où, avec de bons objectils, on peut voir, à côté de la ligne saillante, plus ou moins onduleuse, qui règne au milieu de la valve, une fente très nette. C'est ce que montre la fig. 33 qui représentent une valve brisée de Navicula major, d'après M. H. L. Smith. On voit dans cette valve la fente $c b$ du raphé longeant la ligne flexueuse épaissie et interrompue par le nodule médian $e$.

Quelques auteurs, M. A. Schmidt notamment, nient cependant que le raphé soit une fente. M. Julien Deby n'admet pas de communication directe entre le contenu de la cellule et le milieu extérieur. Il est possible, en effet, que celte fente qui parait incontestable sur les valves siliceuses des frustules fossiles ou traités par les acides, soit oblurée, sur les Diatomées vivantes, par la membrane cellulaire organique. La communication entre le protoplasma interne de la cellule et le milieu ambiant, communication nécessaire aux échanges vitaux, se ferait par endosmose à travers la membrane organique dans les points ou fentes oủ manque le revêtement siliceux.

Quant aux nodules, comme on le voit sur ces deux dernières figures pour le nodule médian, ce sont des épaississements de la couche siliceuse, faisant saillie en dehors ou en dedans $(f, f$, fig. $32 ; e$, fig. 33$)$, et qui peuvent à leur surface extérieure être plus ou moins creusés en cupule. Il en est de mème des nodules terminaux; cependant sur le côté de ces nodules, que contourne ordinairement le raphé, il existe des points, élargissements de la fente de ce raphé, qui traversent l'épaisseur de la valve et par lesquels on a admis jadis que sortaient des cils vibratiles ou des pseudopodes, et, plus récemment, que se produisaient des courants d'où résulteraient les mouvements des Diatomées. La première de ces hypothẻses est aujourd'hui abandonnée des diatomistes, et nous pensons qu'il doit en etre de même pour la seconde.

Le prof. H. L. Smillı a fondé sur la présence ou l'absence du raphé une classiticatịon générale des Diatomées qui a été adoptée par un certain nombre d'auteurs, M. H. van Heurck et M. Ad. Schmidt, notamment. Fondée sur ce seul caractére de forme extérieure, celte classification n'est point ce qu'on appelle une classification naturelle, c'est un système artifi- 
ciel, un peu comme le serait, par exemple, une classification botanique qui répartirait les plantes suivant qu'elles ont les feuilles pointues, rondes ou divisées. Néanmoins, comme elle est employée dans plusieurs ouvrages importants, nous devons en dire ici quelques mots.

M. H. L. Smith divise toutes les Diatomées en trois grand groupes. Le premier, groupe des Raphidées, comprend toutes les Diatomées qui ont un raphé évident, au moins sur l'une des faces. On peut en prendre comme type les Navicula. Elles ont, du reste, ordinairement une forme plus ou moins naviculaire, c'est-à-dire en navette ou en nacelle. (Fig. 27, 1).

Le deuxième, groupe des Pseudo-raphidées, comprend les espèces qui n'ont pas de vrai raphé, mais présentent, au moins sur l'une des valves, un espace lisse simulant un raphé par l'absence des stries, côtes ou dessins. On peut prendre pour type les Synedra. Elles ont souvent une forme plus ou moins bacillaire ou en bâtonnet. (Fig. 27, 2).

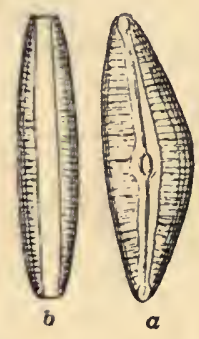

Fig. 34. - Cymbella Ehrenbergii. $a$, vue de profil; $b$, vue de face.

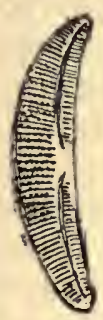

Fig. 35. - Amphora ovalis.

Le troisième, groupe des Crypto-raphidées, se compose des espèces qui n'ont ni raphé ni pseudo-raphé. On peut prendre pour type les Coscinodiscus. Elles ont ordinairement une forme discoïde, ellipsoïde large, triangulaire ou polygonale. (Fig. 27, 3)

En faisant intervenir dans ces trois grands groupes les caractères tirés de la forme des valves, de la position des nodules, etc., M. H. L. Smith établit les coupes de familles, de genres et d'espèces.

Cette classification, on doit le reconnaitre, est fort ingénieuse et attrayante. Malheureusement, elle ne considère dans les Diatomées que la matière morte, comme ferait une classification minéralogique répartissant les objets suivant la forme de leurs cristaux et la direction des axes; elle néglige complètement les Diatomées en tant qu'organismes vivants, ayant les uns avec les autres des ensembles de caractères biologiques communs et des affinités naturelles; elle contribue, par conséquent, dans une certaine mesure, à limiter l'étude des Diatomées à l'examen de leur carapace siliceuse, et à faire négliger les dispositions organiques et les phénomènes 
vitaux que présentent ces curieuses petiles plantes dont l'histoire natırelle est encore, nous le répétons, presque complẻtement à faire.

Quoi qu'il en soit, et en dehors de sa classification proprement dite, I. H. L. Smith a basé sur la division des Diatomées en trois grands groupes, telle qu'il l'établit, des considérations intéressantes que nous devons résumer (1).

Etant données ces trois formes principales, toutes les autres formes en dérivent, suivant le savant auteur américain, à l'aide de variations homologues dans les trois groupes.

Ainsi, dans le premier groupe, par exemple, les valves sont partagées normalement en deux parties symétriques par le raphé, mais dans certains cas, les valves peuvent se développer d'un côté du raphé plus que de l'autre : elles ne sont plus symétriques de chaque côté du petit axe; c'est ce que M. H. L. Smith appelle une variation équatoriale. Le frustule, gon-

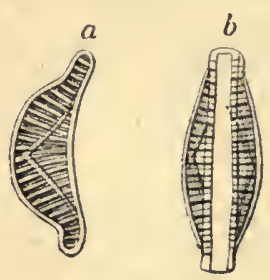

Fig. 36. - Epithemia gibberula. Eh. $a$, face valvaire; $b$, face connective.

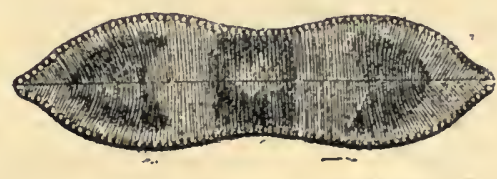

Fig. 37. - Cymatopleura Solea.

flé d'un côté, s'incurve de l'autre. C'est ce qui arrive dans les Cymbella, dans les Cocconema (fig. 34), qui sont des Navicula dans lesquels un côté des valves est plus dilaté que l'autre et la zone connective de ce côté s'est elle-mème élargie. La Diatomée s'est, pour ainsi dire, gonflée de ce côté, qu'on appelle côté dorsal. (L'autre côté, plus étroit et plus mince, est le cóté ventral).

La variation équaloriale peut s'accuser encore davantage que dans les Cymbella, et l'on obtient un Amphora. (Fig. 35).

La même variation, par expansion équatoriale de la zone connective d'un côté de la valve, se produisant dans le deuxième groupe donne les formes Epithemia, (Fig. 36), et dans le troisième groupe les formes Euodia.

La variation au lieu de se produire à l'une des extrémités du petit axe pour se faire à l'un des bouts du grand axe, elle est alors dite axiale.

(1) H. L. Sмгтн. - A contribution to the life history 0! Diatomacex (Proceedings of the Amer. Soc. Microc. 1886). - Traduit dans le Journal de Micrographie, t. XII, 1888. 
C'est ainsi que s'obtiennent les Diatomées cunéilormes comme les Gornphonema dans le premier groupe (Raphidées), Licmophora, Rhipidodendron, etc., dans le second (Pseudo-raphidées) (Fig. 23), Podosira, dans le troisiẻme (sans raphé ni pseudo-raphé).

La dilatation axiale peut se faire aux deux extrémités, et l'on obtient des formes capitées ou dilatées en 8 , dans les groupes qui comportent ces formes (Fig. 37).

D'autres variations se manifestent encore comme la torsion du frustule en S, par exemple dans les Pleurosigma, du premier groupe, torsion qu'on retrouve chez des Nitzschia du second.

Ou bien encore, certains détails de forme peuvent subir des modifications qui établissent des relations entre tel groupe et les deux autres: l'allongement, l'étirement du nodule central en une ligne tranversale, comme chez les Stauroneis, ou longitudinale, comme chez les Colletonema et les Berkeleya, et qui arrive à l'effacement à peu près complet chez les $A m$ phipleura; la disparition du raphé sur une des valves, ou son émigration

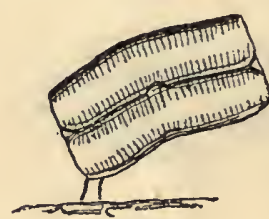

Fig. 38. - Achnantes brevipes.

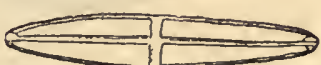

Fig. 39. - Stauroneis gracilis.

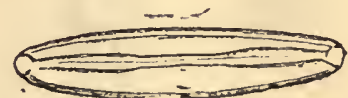

Fig. 40. - Berkeleya Harveyi, Gr. (très grossi).

graduelle vers l'un des bords de la valve, rapprochent les formes du premier groupe de celles des deux autres.

En un mol, en appliquant les mêmes lois de variation aux trois formes normales, on obtient toutes les modifications qui caractérisent les genres et les espèces contenues dans ces groupes, modifications qui procẻdent des mêmes causes.

De plus, on obtient le passage de ces formes les unes aux autrespar des variations graduelles, allant parfois jusqu'à la disparition de certains détails de structure.

Certaines de ces variations se sont fixées, et on en a fait des genres et des espèces, - alors que peut-être ont n'eût dù les regarder que commè des variétés, - mais d'autres ne sont qu'accidentelles, bien que parfois elles puissent se reproduire par la division fissipare des Irustules qui les présentent, et alors elles constituent des formes anormales, de véritables monstruosités.

Ainsi M. Weissflog a publié la photographie de divers Navicula chez lesquels le raphé a disparu sur la moitié d'une valve, (Fig. 41), ou même 
presque complètement (Fig. 42), et oủ les stries composés de grains ou chapelets au lieu de rester parallèles au pelit axe du frustule ont pris une disposition rayonnante. C'est le premier groupe de M. H. L. Smith qui passe au troisième, un Navicula qui devient un Coscinodiscus.

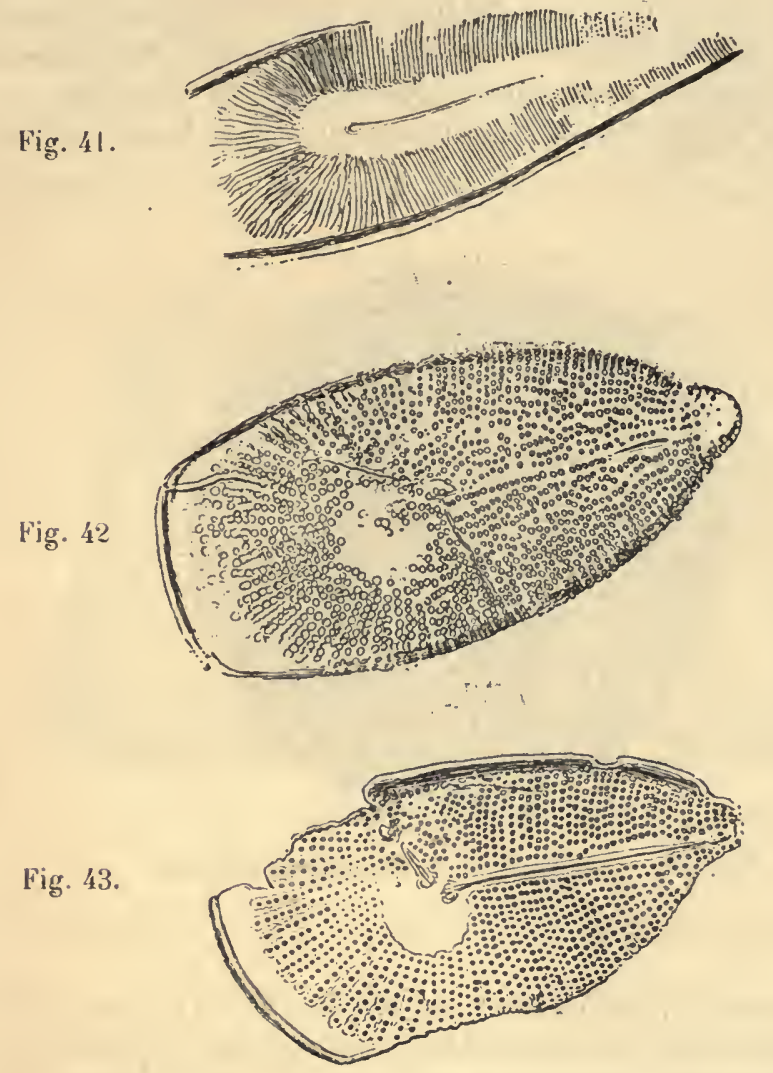

Fig. 41, 4̇̌, 43. - Diatomées anormales (d'après Weissflog).

Dans la forme suivante (Fig. 43), on voit de même un Navicula qui, dans sa moitié inférieure, passe à l'Aulacodiscus.

Nous aurons à décrire par la suite un certain nombre de monstruosités, car les Diatomées, comme tous les autres êtres vivants, sont sujettes à des anomalies, à des variations tératologiques souvent très intéressantes parce qu'elles peuvent nous révéler les différentes phases de développement par lesquelles passent ces organisines, les lois qui président à la formation de leurs parties et les divers états dont ils procèdent. 


\section{§ 2. - STFUCTURE INTIME DES VALVES}

Nous avons vu que les valves des Diatomées présentent des côtes, stries, grains ou perles, des détails de sculpture infiniment variés et délicats. Nous avons donc à examiner maintenant la structure intime de ces valves.

C'est là une question très ardue, très controversée el le plus souvent, du

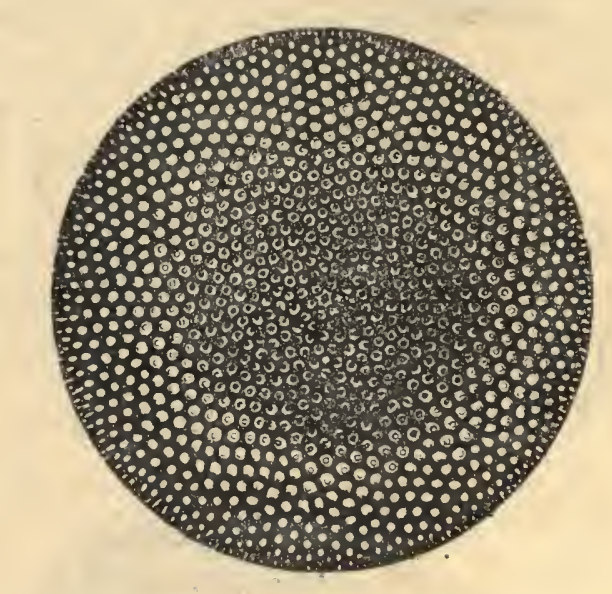

Fig. 44. - Cestodiscus (Coscrnodiseus) obscurus.

reste, extrêmement difficile à résoudre, exigeant toutes les ressources de l'optique moderne la plus perfectionnée, et une grande habileté de la part du micrographe. C'est, comme le dit M. J. Deby dans notre Introduction, le champ de bataille des diatomistes.

Quand on examine, avec un bon objectif, une Diatomée portant des stries sur ses valves, on reconnaît que, le plus souvent, les stries se résolvent en lignes de points, et tous ces points, au premier abord, paraissent comme des grains saillants, aussi les a-t-on généralement appelés perles.

Cependant, en y regardant de plus près, sur certaines espèces, comme les Triceratium, les Coscinodiscus, les Isthinia et d'autres dont les points sont plus gros, on se prend à douter, et, avec beaucoup d'attention, on admet que ces points ne sont pas en saillie, mais en creux.

C'est, pour nous, une disposition à peu près évidente sur le Coscino- 
discus Oculis Iridis (1), entr'autres, où nous avons pu constater que les' hexagones ou yeux dont sont marquées les valves ont leur centre sur un plan inférieur à celui du périmètre hexagonal. Cela est si vrai qu'en abaissant très légèrement l'objectif, on reconnait un dessin très fin que nous avons pu représenter. De sorte que le centre de l'hexagone est le fond d'un alvéole et non le sommet d'une perle. C'est le fond qui porte une sculpture extrêmement fine, que l'on peut reconnaitre en abaissant l'objectif pour mettre ce fond au foyer.

Dès 1876, nous constations ainsi que la valre des Coscinodiscus, et autres espèces dont le dessin est analogue, est composée de deux couches, la première formée d'aréoles ou alvéoles, plus ou moins semblable á un

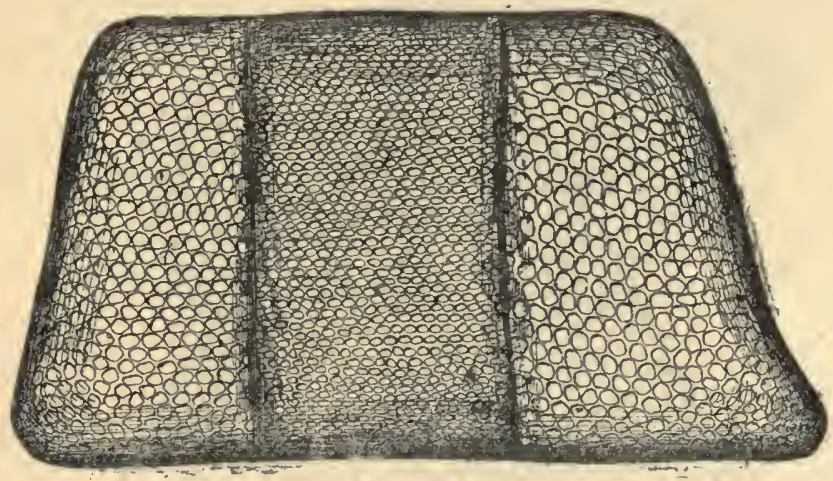

Fig. 45. - Isthmıa enervis.

gâteau d'abeilles, et la seconde plus profonde fermant ces alvéoles par le fond.

C'était presque la vérité, comme nous le verrons plus loin.

C'est, à ce que nous croyons, le professeur Bailey, de New-York (2), qui a démontré, en 1851, que la plupart des parties que l'on prenait alors pour des trous ou des dépressions des valves sont, au contraire, des épaississements, et qu'inversement les parties que l'on regardait comme épaissies ou saillantes sont, en réalité, amincies ou déprimées.

Par exemple, ot considérait les nodules des Naviculées comme des trous et les points des Isthmia comme des perles ou des bosses.

Bailey a eu l'idée de faire agir sur les frustules une solution d'acide fluorhydrique, qui dissout la silice. Il opérait sous le microscocope, en plaçant

(1) J. Pelletan. - Le Microscope, son emploi et ses applications, in-8 ${ }^{\circ} 1876$ p. 563.

(2) J. W. BAIlex, - Amer. Journ. of Sc. and Arts, $2^{2}$ série, tome XI. 
les frustules entre deux lames de mica que l'acide fluorhydrique n'attaque pas, et en recouvrant d'une semblable lame de mica la lentille de l'objectif pour la préserver des vapeurs acides. Il a vu ainsi que, sur les valves présentant des nodules, ceux-ci résistaient le plus longtemps à l'action dissolvante du fluor, tandis que s'ils eussent été des parties plus minces ou des trous ils eussent disparu les premiers ou se seraient agrandis.

Il a vu, de même, que les bandes longitudinales lisses, de chaque côté du raphé dans les Pinnularia, ne sont pas des parties amincies, mais épaissies, et qu'au bout d'un certain temps, l'acide ayant attaqué la surface, elles apparaissent striées. Cette striation est donc profonde et recouverte par une couche de silice.

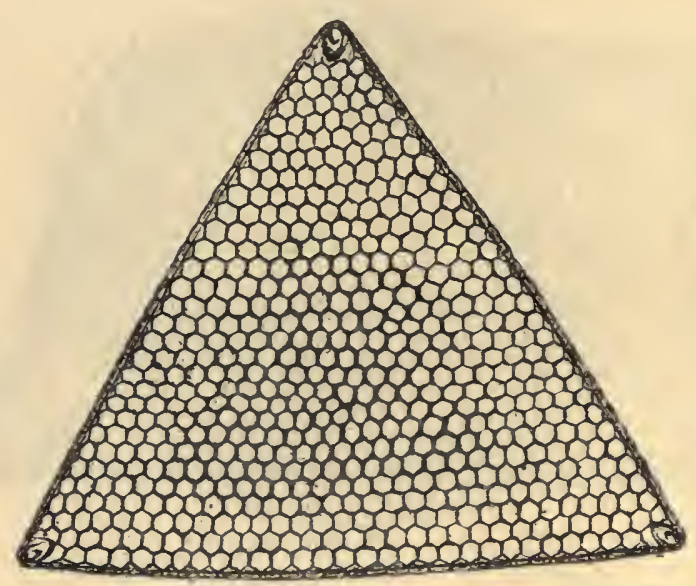

Fig. 46. - Triceratium favus.

Il a constaté encore que, chez les Isthmia, les taches ou points de la bande transversale de la valve ne sont pas des épaississements ni des bosses, mais des parties amincies ou des trous arqués, et qu'au contraire, les travées qui les séparent, et que l'on croyait tracées en creux, sont des épaississements qui résistent longtemps à l'action de l'acide et disparaissent en dernier (Fig. 40̃).

Par ce procédé ingénieux, il a pu, sur les frustules récents et même sur quelques spécimens fossiles, isoler la membrane organique de la cellule dèpouillée de toute son incrustation siliceuse.

Depuis Bailey, un grand nombre d'observateurs ont étudié la structure intime des valves des Diatomées, et ce sont particulièrement sel valves des Coscinodiscus et des Triceratium, dont les "yeux " sont relativement volumineux, qui ont été l'objet des recherches des diatomisles. Il faut citer 
surtout les travaux de Flögel (1), Otto MüHer (2), J. Cox (3), Prinz et Van Ermengem (4), Van Heurck (5) et J. Deby (6).

Aujourd'hui, tout le monde est à peu près d'accord à reconnaitre que les valves des Diatomées, et particuliẻrement de celles que nous venons de citer, sont composées de plusieurs couches, une couche supérieure et une couche inférieure réunies par un réseau composé d'alvéoles plus ou moins réguliers et souvent hexagonaux, formant comme un diploë entre deux lames osseuses.

Il arrive parfois d'ailleurs qu'une valve de Diatomée se clive en ses deux lames parallèles. M. J. Cox a pu reconnaître ainsi directement que la lame inférieure ou interne de la valve d'un Triceratiuım portait les fines ponc-

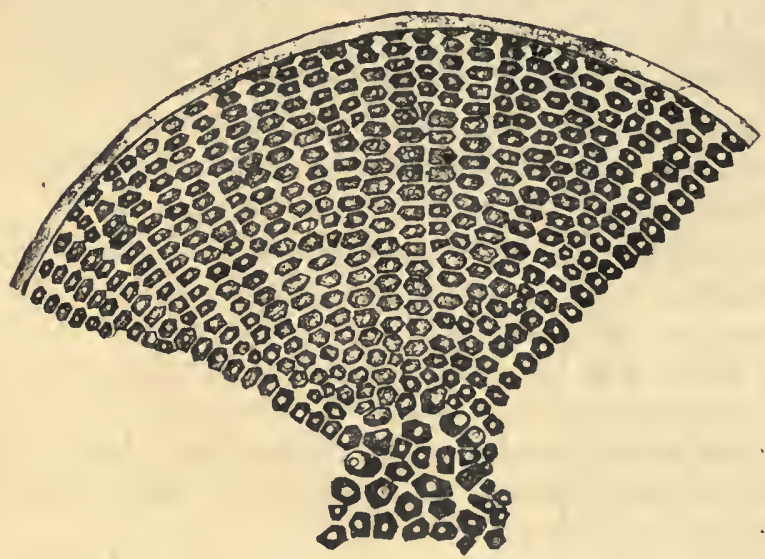

Fig. 47. - Coscinodiscus bihariensis, Pant.

tuations que l'on voit au fond des yeux quand on examine la valve dans son entier, et l'on reconnaissait la trae hexagonale laissée par le réseau alvéolaire qui était resté adhérent ả la face inférieure de la lame externe (7).

(1) Fü̈GEL. - Untersuchungen über die Structur der Zellwand, etc. (Arch. f. Mikr. Anat. T. VI, 1870).

(2) 0. MüLler. - Uber der feinerem Bau der Zellwand der Bacillariaceen, etc. (Arch. f. Physiol. Reich. et Dub. Reym. 1871),

(3) J. Cox. - Structure of the Diatom shell. (Am. Month. Micr. J. 1884).

(4) M. Prinz et Van Erm engem. - Rech. sur la struct. de qq Diat. cont. dans le cementstein du Jutland. (Ann. de la Soc. B. de Micr. T. 8).

(5) H. Van Heurck. - Bull. de la Soc. B. de Mier. 1885.

(6) J. Deвy. - Sur la structure microscopique des valves des Diatomées. (Journal de Micrographie, 1836, et Journ. Quek. Micr. Cl. 1886.)

(7) J. DrsвY. - Sur la structure microscop. des valves des Diat. (Journ. de Micrographie, 1886 p. 416). Voir la Pl. IV, fig. 4, 5, 6, qui représentent les lames sup. et inf. et la coupe d'une valve de Triceratium. 
Néanmoins, certains auteurs, comme MM. Prinz et Van Ermengem, pensent que la lame supérieure est perforée au centre des alvéoles qui sont ainsi des cavités ouvertes èn dessus comme dans un gateau d'abeilles vide. M. Stephenson, au contraire, avait supposé que c'était la lame inférieure qui portait la perforation.

Le $\mathrm{D}^{\mathrm{r}}$ Flögel et M. J. Cox pensent que les al véoles sont des cavités closes par le fond et le $\mathrm{D}^{\mathrm{r}} \mathrm{H}$. Van Heurck admet qu'une lame supérieure peut fermer les alvéoles en dessus, mais qu'elle existe à tous les états de développement : complètement formée, épaisse et solide, obturant entièrement les alvéoles, ou bien très mince et même manquant tout à fait, et laissant les alvéoles ouverts en dessus.

M. J. Deby qui, à ce que nous croyons, a fait les recherches les plus récentes sur ce sujet, pense que la couche ou membrane silicifiée supérieure existe toujours, à moins qu'elle ait été détruite accidentellement, comme cela arrive presque toujours sur les frustules fossiles qui ont subi des frottements et qui se sont usés sur leur surface, et comme cela a lieu aussi à peu près constamment sur les Diatomées qui ont été préparées par le brûlage ou traitées par les acides.

L'observation que nous avions faite jadis sur lo Coscinodiscus Oculus Iridis était donc exacte, - comme sont exactes celles de MM. Prinz et Van Ermengem, mais, ainsi que ces auteurs. nous avons étudié une Diatomée fossile dont la couche externe, usée, avait disparu, laissant les alvéoles ouverts.

Voici, d'ailleurs, les conclusions du travail de M. J. Deby.

" $1^{\circ}$ La valve de la plupart des Diatomées est composée d'une double lame.

- $2^{\circ}$ Entre les deux lames il y a un plus ou moins grand nombre de cavités limitées pâr des parois solides de silice. Ces cavités sont circulaires ou hexagonales dans leur contour.

- " 3 ० Dans toutes les valves récentes vivantes et complètes, les cavités sont closes en-dessus par la lame supérieure, au fond par la lame inférieure, et ces lames ne montrent aucune trace d'orifices, mais seulement des amincissements au sommet des cavités, excepté dans. les cas anormaux ou la cuticule organique a été partiellement ou totalement détruite par des causes accidentelles.

" $4^{\circ} \mathrm{La}$ membrane supérieure est, dans le plus grand nombre des cas, si légèrement siliceuse, que le moindre contact avec les acides la détruit et ouvre les cavités placées au-dessous d'elle. Dans d'autres cas, cette membrane, qui est généralement plus mince dans la partie centrale des aréoles, peut devenir fortement silicifiée et contenir des particules ou granules de silice fortement réfringents, placés sur ce qu'on appelle les " yeux " auxquels cas les cavités sont complètement fermées des deux côtés, sauf pour les actions osmoliques.

- $3^{\circ} \mathrm{La}$ membrane de clôture inférieure des alvéoles porte fréquemment. 
des dessins variés dont la nature, en raison de leur excessive petitesse, n'a pas encore été bien établie, mais qui doivent dépendre de la structure, car aucune image de diffraction, formée par queiqu'organisation siégeant sur le plan inférieur, ne peut les produire, attendu qu'aucune organisation n'existe sous ce fond, ni entre les diaphragmes.

" $6^{\circ}$ La fine membrane supérieure des aréoles est l'extension des bords des barres dites " en tête de clou " qui forment les parois limitantes des aréoles, comme cela a été figuré par MM. Otto Müller, Flögel, Prinz et Van Ermengem. (Dans beaucoup de Diatowiées fossiles et sur presque tous les spécimens bouillis dans les acides, la lame externe qui ferme les aréoles a disparu et les valves sont, par conséquent devenues perforées à la surface supérieure. Dans quelques cas, la lame profonde finit aussi par présenter des perforations.)

- $7^{\circ}$ Les cavités dans la valve sont limitées par des parois de silice solide.

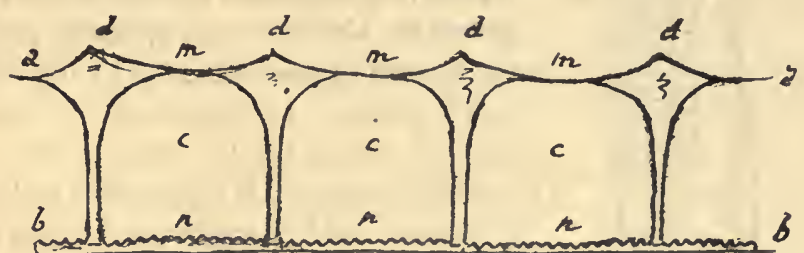

Fig.48. - Coupe schématique d'une valve de Triceratium, d'après M. J. Deby.

$a$, lame supérieure ; $b$, lame inférieure ; $c$, cavité des alvéoles $; d$, piliers interalvéolaires; $m$, partie amincie de la lame supérieure ; $n$, fond des alvéoles.

Ces parois s'étendent souvent au-delà, en-dessus ou en-dessous des membranes qui ferment les aréoles, et fréquemment s'allongent en pointes' ou épines, diverses de formes et de longueur, qui font saillie sur la valve entre les aréoles.

- $8^{\circ}$ Le sillon médian ou la fissure qu'on observe dans le raphé, ou ligne médiane épaissie de la plupart des Navicula, est aussi fermée en-dessus et en-dessous par une membrane organique très mince, légèrement silicifiée, dans toutes les valves récentes normales. Je crois cependant que de petites ouvertures peuvent exister dans ces étroites membranes de fermeture au voisinage du nodule central et des nodules terminaux. Mais ce point a besoin d'ètre encore élucidé....

" $9^{\circ}$ Les zones ou bandes connectives de certains genres, comme les Isthmia, paraissent réellement et véritablement periorées.

"10 ${ }^{\circ}$ Tout ce que les auteurs ont appelé aréoles, perles, pores, orifices, projections granuleuses, dépressions, hexagones, grains en chapelet. points, etc. ne sont qu'une seule et même chose mais exprimée d'après des 
interprétations microscopiques diverses, des idiosyncrasies différentes ou des idées préconçues. "

Ainsi, comme nous le disions, les Diatomistes paraissent s'accorder aujourd'hui sur l'existence de deux lames séparées par une substance aréolaire, lames dont l'externe ou supérieure peut manquer souvent, sans doute par suite d'usure ou de destruction accidentelle.

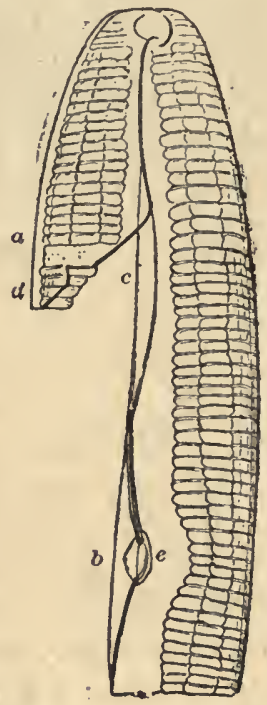

Fig. 49. - Navrcula major

raphé et nodules.

$a d$, fracture monmontrant la lame supérieure recouvrant les côtes. - Deux côtes manquent.

D'ap.M.H.L.Smith).

Pour certaines Diatomées, comme les Coscinodiscus, Eupodiscus, Triceratium, elc., cette structure paraît assez évidente et elle donnerait, sur une coupe perpendiculaire à la surface, le schéma ci-dessus (fig. 48) dans lequel $a$ représente la lame externe ou supérieure, $b$ la lame interne pouvant porter des ornementations diverses, $c$ la cavité close des alvéoles, $d$ les piliers en tête de clou qui séparent les alvéoles et $m$ la partie amincie de la lame supérieure dans l'axe des alvéoles, partie à travers laquelle, si elle est conservée. (ou par le trou qu'elle laisse, si elle est détruite), on aperçoit le fond orné $n$ de l'alvéole.

Mais en est-il de même pour toutes les Diatomées, par exemple pour celles dont les valves ne présentent pas d'yeux, mais des côtes plus ou moins robustes comme les Navicules Pinnulariées, et pour celles qui présentent des stries résolubles, avec de forts grossissements, en points distincts lesquels ont bien l'aspect de grains brillants ou, comme on dit, de perles, les Pleurosigna, Frustulia, Amphipleura, et mille autres? - La chose parait bien moins évidente, et d'autant moins que ces détails de structure sont ordinairement beaucoup plus petits et souvent très difficiles à voir.

Il nous semble que jusqu'à présent ce n'est guère que par analogie qu'on a conclu à une identité de structure chez toutes les Diatomées. Cependant, M. H. Van Heurck, grâce aux milieux réfringents à haut indice de réfraction composés par M. H. L. Smith (voir plus loin : Montage des Diatomées), a pu vérifier l'existence des alvéoles dans les genres Raphoneis, Nitzschia et Pleurosigma.

Pour nous, nous ne contestons pas la réalité de cette texture aréolaire des valves chez les espéces à fines stries résolubles en points, non plus, par conséqquent, que l'existence des deux lames, l'une supérieure fermant les 
alvéoles en dessus, l'autre inférieure fermant les alvéoles aı fond. Mais nous pensons que les points en lesquels se résolvent les stries sont bien des grains ou des perles, c'est-ḋ-dire des corps en saillie et non plus en
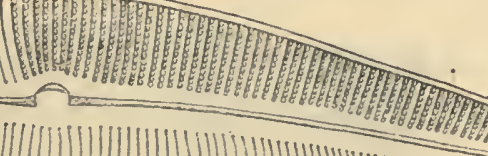

Fig. 50. - Cocconema asperur, Ehb.

Face valvaire montrant les stries résolues en perles dans une partie de la valve, d'après MM. P. Petit et Leuduger-Fortmorel.

creux ou en profondeur comme les yeux ou les taches des Coscinodiscus ou des Triceratium.

En effet, si l'on se reporte anx figures qu'ont données les auteurs de la

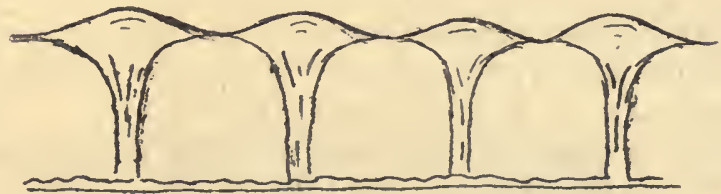

Fig. 51. - Coupe schématique perpendiculaire

à la surface d'une valve perlée.

coupe des valves (voir fig. 48), on remarque que tous ont représenté les piliers interalvéolaires sous forme de clous á tẻte saillante. C'est, en effet,

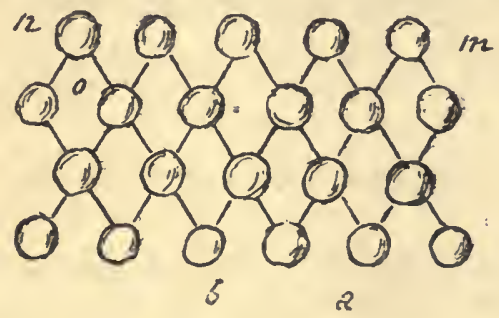

Fig. 52. - Schéma de la strialion perlće du Pleurosigma angulatvm.

ainsi qu'ils se présentent toujours. Dans les espèces dont nous parlons, Pleurosigma et autres, les alvéoles sont extrêmement petits et les têles de clous saillantes relativement grosses. Ce sont précisément ces têtes de 
clous qui constituent les grains ou perles, réellement en saillie, qu'on observe sur les valves des espéces à surlace perlée.

Si les alvéoles (o) ont une section régulière en losanges égaux disposés, par exemple, comme dans le schéma fig. כ22, sur toute la surface de la valve,

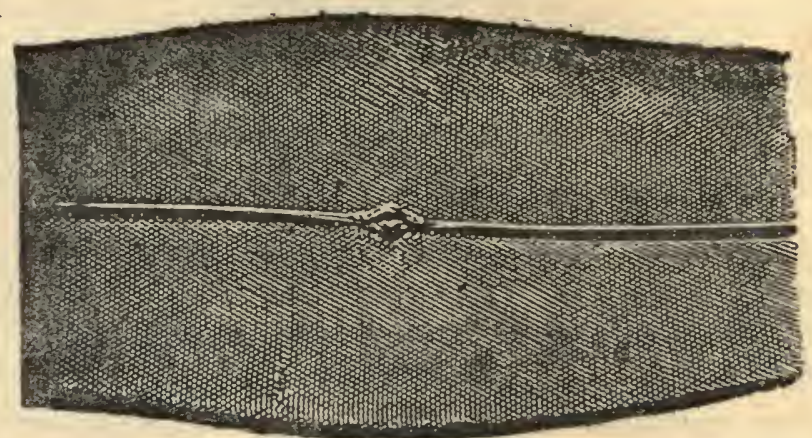

Fig. 53. - Pleurosigma angulatum résolu en perles à la lumière électrique, par M. H. Van Heurck.

on voit qu'ils forment exactement le dessin des stries du Pleurosigma angulatum résolues en perles, alignées suivant les trois directions $m n$, transversale, $m a$ et $n b$, inclinées l'une sur l'autre a $60^{\circ}$.

Les alvéoles peuvent avoir un contour carré ou rectangulaire allongé, et l'on obtient ainsi le schéma de toutes les striations perlées disposées en
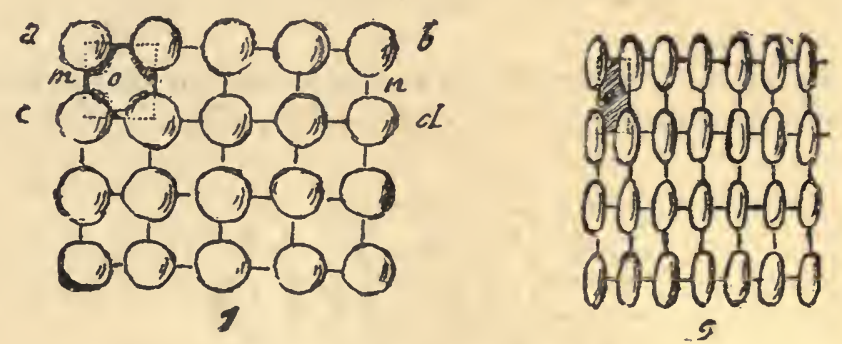

Fig. 54. - Schémas de striations parallèles perlées.

en stries parallèles, comme dans un grand nombre de Navicula, dans les Frustalin saxonica, Amphipleura pellucida, dans le Surirella gemma, etc.

Les alvéoles peuvent avoir des formes très diverses suivant la forme même de la valve et former, par les têtes saillantes des piliers qui les séparent, des lignes, c'est-à-dire des stries, très diversement inclinées les unes sur les autres, et par exemple plus ou moins rayonnantes. 
Cette disposition s'observe, comme on sait, chez un très grand nombre de Diatomées et particulièrement dans le voisinage des nodules. (Fig. อ̆5̆, こ̋6).

Si l'on suppose que les grains formant une strie sont tellement près les uns des autres qu'ils se touchent, confluent, la strie ne sera plus résoluble en grains ou perles, mais ne formera plus qu'une barre saillante ou côte

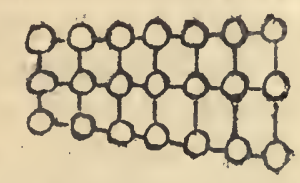

Fig. 55. - Schéma d'une striation perlée rayonnante.

séparée de la barre ou côte précédente et de la suivante par une raie en creux.

Beaucoup de Diatomées présentent des stries qui n'ont pas encore élé résolues en grains. Pour quelques-unes il est possible que les moyens optiques nous manquent encore, mais il parait très probable que chez d'autres les grains n'existent pas, qu'ils ne sont pas distincts les uns des autres et qu'ils confluent en une strie irréductible. On donne, en général, le nom

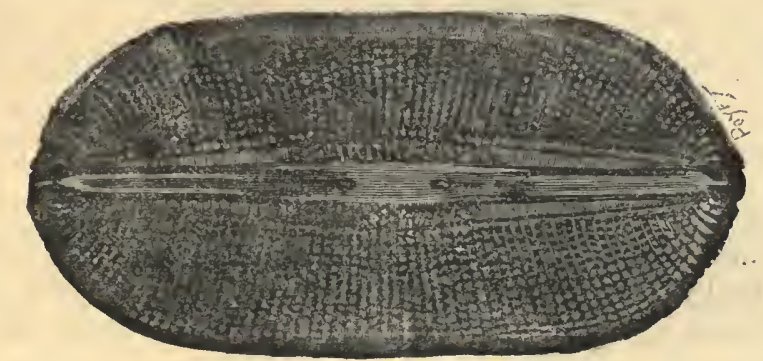

Fig. 5f. - Navicula ıusca résolu en perles à la lumière électrique par M. Van Heurck.

de côtes à ces stries lorsqu'elles sont très fortes, comme dans les Naricula major (Fig. 49), N. Debyi (Fig. 577), etc.

Du reste, il n'est pas certain que ces côtes soient toujours formées, comme nous venons de le supposer, par la confluence des sommets des piliers de séparation des alvéoles. Dans certains cas, elles paraissent formées par des sortes de petits tubes aplatis rangés les uns à côté des autres et qui seraient creusés le long de leur axe d'un canalicule très fin. Nous considérons cette structure comme résultant de la coalescence des cavités d'une ou plusieurs rangées d'alvéoles. 
Ainsi, si l'on se reporte à la fig. כ̋́, et que l'on suppose que les perles des deux premiers rangs soient assez grosses pour se confondre entr'elles dans le rang $a b$ et dans le rang $c d$ et celles du premier rang avec celles du second de sorte que les deux rangs $a b, c d$ ne forment plus qu'une grosse côte épaisse et saillante; si l'on suppose que le rang d'alvéoles compris sous ces rangées de grains confluent aussi, par la disparution de toutes lescloisons comme $n, n$, le rang d'alvéoles formera un canalicule qui sera surmonté par la côte composée de la fusion des deux rangées de grains.

En somme, cetle structure à côles canaliculées ne serait, ainsi comprise, qu'une modification de la structure alvéolaire supposée générale á toutes les valves de Diatomées. Elle en dériverait d'une manière simple. De sorte que dans une même tribu, comme celle des Naviculées, ou même, plus généralement, dans un même groupe, comme celui que M. H. L.

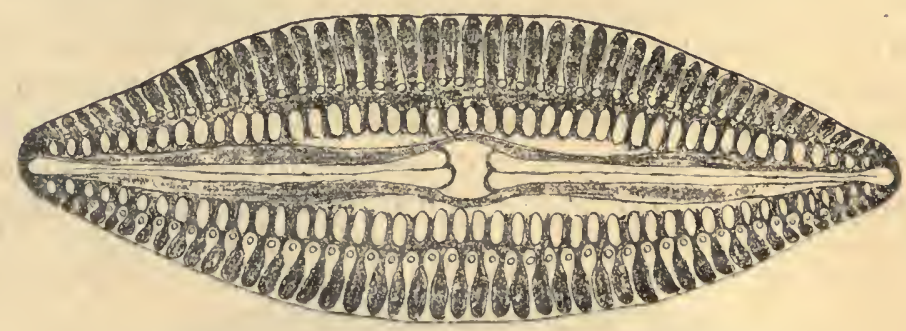

Fig. 57. - Navicula Debyi.

Smith appelle les Raphidées, on ne trouverait pas des espèces dont la valve est construite de manières très différentes. Et, dans tous les groupes, la structure fondamentale serait la même, ne différant que par des variations dạns la forme, la dimension, la séparation ou la réunion des mêmes éléments.

Nous avons parlé de valves de Triceratium qui se sont dédoublées en une lame supérieure, à laquelle adhéraient les alvéoles, et une lame inférieure montrant encore l'empreinte de la réticulation hexagonale laissée par ces alvéoles; M. H. L. Smitt a publié le dessin (Fig. 49) d'une valve de Navicula major brisée et montrant, dans la fracture $a d$, la lame supérieure passant par-dessus les côtes qui la débordent en un point et qui sont fixées à la lame inférieure. Deux côtes manquent tout à fait par la disparution des deux rangs d'alvéoles qu'elles recouvraient.

La manière de voir que nous exposons ci-dessus n'est pas, nous le savons, admise par tous les auteurs. Beaucoup pensent que les stries perlées ne sont pas en saillie. Ainsi le $\mathbf{D}^{r} \mathbf{H}$. van Heurck, d'après des études faites sur des valves examinées dans des milieux à très haut indice de ré- 
fraction, considère ces stries comme formées non pas par les lêles des piliers interalvéolaires, mais par la vue en coupe optique d'une série d'alvéoles. Il assimile, par conséquent, les points qui composent une strie de Navicula, d'Amphipleura, de Nitschia, de Surirella, etc., à ceux qui forment les " yeux "des Triceratium ou des Coscinodiscus. Ainsi, en se reportant à la fig. 54 , ce serait la ligne $m n$ qui représenterait la

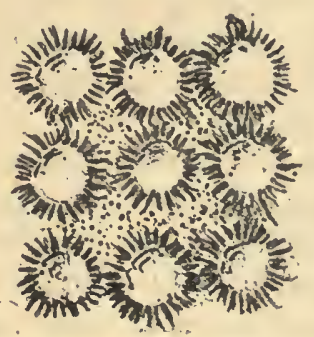

Fig. 58. - Perles grossies à 2000 diamètres, d'un Stauroneis.

strie dont les points seraient formés par la cavité des alvéoles eux-mémes, cavité aperçue à travers la fine lame supérieure si elle existe, ou vue directement en creux si cette lame est détruite ; les cloisons séparant les lignes d'alvéoles, cloisons qui seraient saillantes ou épaissies, ne formeraient que des lignes uniformes que M. van Heurck appelle interstries (1).

Nous avons indiqué les raisons qui nous font croire qu'il n'en est pas

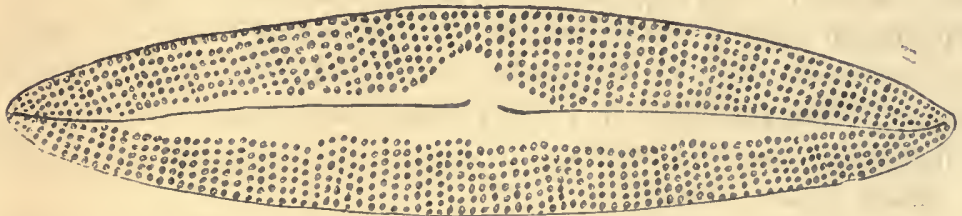

Fig. 59. - Alloioneis Antillarum. Cl.

ainsi et que les stries perlées sont bien formées par des grains en saillie, dilatation ou épaississement des " têtes de clous. "Nous ajouterons seuloment que lorsqu'on examine ces "perles, "au microscope, sur de gros spécimens, l'œil qui a une si une grande habitude de discerner les reliefs et les creux perçoit complètement la sensation de relief. D'ailleurs, certaines Diatomées présentent des grains et des détails de structure qui permettent de les voir assez distinctement avec le microscope binoculaire ; on

(1) H. vax Hexac - Synopsis des Diat. de Belg. p. 244. 
peut alors apprécier très nettement la saillie que forment ces grains, saillie qui a depuis longtemps fait comparer ces frustules à une rape. (Hig. 59).

Enfin, l'aspect brillant, la réfringence de ces points, quand on les examine à sec, indique bien que ce sont des parties épaissies, et réfractant plus la lumière, en raison de leur épaisseur même et de leur forme bombée, que les parties avoisinantes, lesquelles ont cependant le même indice de réfraction, étant formées de la même substance, une silice transparente, mais sont moins épaisses. Ce sont bien ces sommets bombés qui se piquent d'une étincelle de lumière quand on les éclaire fortement par des rayons très obliques, étincelle qu'on retrouve sur clıaque perle dans les images photographiques. (Voir Fig. 533, วั6).

Et si l'on se fonde, pour établir que les grains sont des "creux", sur l'examen des valves dans des milieux à plus haut indice de réfraction que la silice dont ils sont constitués, on a tort, car on démontre précisément le contraire de ce qu'on veut prouver, les granules devant paraitre des creux en raison de leur moindre réfringence. Quand on regarde un objet transparent dans un milieu moins réfringent que lui, cet objet apparaît plus ou moins brillant, mais avec son relief; dans un milieu de même réfringence, il dísparait complètement et on ne le voit plus; dans un milieu plus réfrin. gent il apparaît comme un vide ou un trou.

A côté de ces diverses opinions sur la nature réelle des stries des Diatomées, il y en a encore une, trẻs radicale, que nous devons signaler. C'est que ces stries n'existent pas du tout, ou, au moins, n'existent pas telles que nous les voyons.

Et, en effet, le professeur Abbé a démontré, comme nous l'indiquerons plus loin (voir : Objectifs), que, suivant les conditions optiques dans lesquelles on examine, au microscope, les objets à très fine structure, on peut obtenir des images très différentes, et particulièrement très différentes de ce qui existe en réalité. M. J. Deby estime que le diatomo-microscopiste ne doit jamais perdre de vue les phénomènes de diffraction indiqués par M. Abbé.

En thèse générale, cela est vrai, et il est possible, après tout, que les images que nous connaissons des structures extraordinairement fines de certaines Diatomées difficiles, comme l'Amphipleura pellucida, le Surirella gemma et quelques autres, ne représentent pas ce qui existe réellement sur les valves de ces espèces. Cependant, cela est peu probable.

Il faut, en général, se méfier des raisonnements par analogie. Toutelois, il est évidemment des cas oủ ce genre de raisonnement parait fondé. Ainsi, comme le fait très bien remarquer M. H. van Heurck, nous connaissons beaucoup de Diatomées dont la structure striée ou perlée est assez grosse et nette pour qu'on puisse la distinguer parfaitement, à peu près avec tous les objectifs. C'est, en un mot, une structure connue, toujours la même, et nous pouvons êlıe certains qu'elle existe réellement telle que, nous la 
voyons toujours. A côté de ces espéces, il y en a une série d'autres, voisines, présentant une structure semblabfe, mais de plus en plus fine; ce n'est que dans les derniers spécimens de cette série, oủ les détails deviennent extrêmement fins, que l'on pourrait avoir des doutes sur la réalité des images et supposer qu'elles ne sont, peut-être, que des effets de diffraction. Mais, comme entre ces dernières espèces et les premières on trouve tout une suite de formes intermédiaires, dont la structure est ceriaine et analogue, on est conduit à admettre par analogie, et avec une raison (jui parait suffisante, que la même structure existe bien aussi, quoique de plus en plus fine, sur les derniers termes de la série, et telle que les objectifs actuels nous la montrent en effet.

D'ailleurs, même sur ces tests difficiles, la structure se inontre toujours la même, pour une même espẻce, quel que soit l'objectif que l'on emploie, dès qu'il présente des conditions optiques suffisantes pour la résoudre. A vec des objectifs d'ouverture numérique et de grossissements différents, on voit la structure ou on ne la voit pas : si on la voit, elle est plus ou moins distincte, mais elle est la mème, dans les mèmes conditions d'éclairage et de milieu.

Nous croyons donc yue la structure des Diatomées, telle que nous la voyons sur les espèces aujourd'hui connues, est une structure réelle, bien que nous pelisions, avec M. J. Deby, qu'il est toujours utile de se rappeler les phénomènes mis en lumière par le professeur Abbé à propos des effets de la diffraction et des images illusoires, surtout quand il s'agit d'interpréter des faits noureaux.

Noubre et constance des stries. - Une question se présente maintenant à laquelle il nous paraît qu'il n'est pas encore possible de répondre d'une manière complète et certaine.

Le nombre des stries sur tous les individus d'une mème espèce est-il c onstant?

Si tous les individus d'une mème espéce avaient la mème taille, on pourrait jusqu'à un certain point préjuger la question et admettre que le nombre de stries ne doit pas changer, ou sans doute, fort peu, sur leurs valves; - bien que, cependant, les Diatomées se présentent comme des organismes très sujets aux variations, et peut-étre serait-ce non pas les individus d'une même espẻce que l'on devrait comparer, mais ceux d'une mème variété.

Mais les individus d'une même espẻce et d'une même variété, ceux qui descendent d'un meme frustule par des divisions successives, peuvent avoir des tailles très différentes, comme on le sait, et varier du simple au double.

Un frustule strié, double d'un autre, présente-t-il sur ses valves un nombre double de stries, celles-ci ayant le nieme écartement sur les deux 
frustules? - Ou bien présente-1-il le même nombre de stries, celles-ci étant deux fois plus écartées que sur le petit frustule?

La réponse à cette question n'est pas, disons-nous, complétement certaine, car les différents auteurs ne sont pas d'accord sur le nombre des stries qu'ils indiquent pour une espéce donnée. Nous-mêmes, nous avons, il y a quelques années, compté les stries sur les valves de quelques Diatomées tests et si les chiffres auxquels nous sommes arrivé s'accordent a peu près avec ceux que donne M. H. Van Heurck, ils ne ressemblent pas du toủ à ceux qu'a trouvés M. Castracane, de Rome, qui a fait tout un long travail sur ce sujet.

Nous n'élevons aucune prétention sur la valeur de nos chiffres, ayant tout simplement fait le comptage, à l'œil, dans le microscope, à l'aide d'un micromètre objectif comparé avec un oculaire divisé, par un procédé bien connu et tout-à-fait classique. Nous pensons, néanmoins, qu'il y a dans le nombre des stries des différences individuelles, particulièrement dans le groupe si éminemment variable des Naviculées dans lequel un si grand nombre d'espèces s'accompagnent de tant de variétés.

Toutefois, d'après l'accord des diatomistes à indiquer le nombre des stries dans une longueur donnée comme un caractère pouvant servir à différencier certaines espèces - ou variétés - voisines, il est évident que la plupart de ces auteurs voient dans ce nombre un élément d'une certaine fixité.

Ainsi, une partie de la question que nous posions tout-â-l'heure se trouve résolue, c'est-à-dire que le nombre des stries serait très variable sur les différents individus d'une même espèce, suivant leur taille, et qu'un frustule de grandeur double présenterait sur ses valves un nombre total double, ou à peu près, de stries, tandis que le frustule deux fois plus petiten présenterait à peu près deux fois moins dans sa totalité. - Ce qui serait fixe, ou à très peu de choses près, c'est le nombre de stries dans une longueur donnée, et l'on emploie ordinairement le centième de millimètre comme mesure.

Nous disons "á peu près, " parce que tout en reconnaissant la constance du nombre des stries dans une longueur égale prise sur les valves d'individus de la même espèce, nous admettons néanmoins une certaine variation due aux différences individuelles.

C'est ainsi que M. H. van Heurck indique 34 à 35 stries dans 1/100 de millim. sur le Frustulia saxonica, il en trouve environ 28 sur e Navicula rhomboides, alors que M. Castracane en indique $\mathbf{2 4}$; il en compte 23 1/2 à 26 sur le Nitzschia sigmoïdea, alors que M. Castracane n'en trouve que $10 / 2$.

Nous n'insisterons pas davantage sur cette question; comme nous le disions plus haut, la solution en est encore un peu douteuse, et nous ne pouvons la formuler qu'en disant que le nombre des stries sur un espace donné est considéré comme fixe dans une même espèce, avec des varia- 
tions assez légères imputables, selon nous, à des différences indiviūuelles et aux erreurs personnelles d'observation.

II. Castracane considère ce nombre de stries comme ayant une valeur importante dans la caracléristique des espèces, et, en effet, on ne peut nier que cet élément n'ait une importance réelle quand il présente des différerices considérables et, pour ainsi dire, typiques. Par exemple, une discussion s'est élevée jadis entre les diatomistes à propos de l'idendité ou de la non idendité de trois " espèces "les Navicula rhomboides, Navicula crassinervia et Frustulia saxonica (1). L'auteur italien estime que ce sont bien trois espèces distinctes en se fondant sur la comparaison du nombre des stries dans 1/100 de millimètre. En effet :

Le Frustulia saxonica a 34 stries transversales et 36 longiludinales.

Le Navicula rhomboides : 24 "

Le Navicula crassinervia: 14 ,

,

17

24,

Ce qui représente évidemment des striations de types très différents.

Lu raison de l'importance qu'il attache à cette donnée, M. Castracane a entrepris un long travail de comptage sur un très grand nombre de Diatomées (2). Le procédé mis en ouvre par lui présente de sérieuses garanties d'exactitude. Il a photographié les Dialomées sous un grossissement toujours le même (כ33క̉ diamètres) et s'est servi des épreuves négatives sur verre pour projeter, à l'aide d'un appareil convenable, les images, qui se trouvaient ainsi considérablement grandies, sur un écran. Il superposail alors à l'image amplifiée une feuille do papier donnant exactement la mesure de 1/100 de millim. prise de même sur l'image d'un millimètre divisé en $\mathbf{1 0 0}$ parties et grossie de la même quantité ; il marquait les stries sur le papier et les comptait (3).

Nous donnons ci-dessous, à titra de document, les nombres auxquels il est arrivé, et nous en ajoutons quelques-uns dus, à M. H. Van Heurck ou que nous avons obtenus nous-mêmes. Pour éviter les fractions, tous les nombres ont été multipliés par 100, et représentent, en conséquence, le nombre des stries existant sur une longueur de 1 millimétre.

(1) Ces trois espèces sont réunies par M. II. Van Heurck, dans sa Synopsis, dans le genre Vanheurckia, et les deux dernières sont portées comme une variété du $V$. rhomboïdes, sous le nom de $V$.crassinervis.

(2) Comle F. Castracane. - Sur les stries des Diatomées et sur la valeur qu'il faut attribuer à leur nombre, etc. (Comm. à l'Ac. dei Nuovi Lincei. Journal de Micrographie, t. 111, 1879).

(3) M. Castracane ne dit pas que l'écran était toujours placé à la même distance, nous pensons qu'il en étail ainsi, car c'était une condition indispensable. 


\section{NOMBRE DE STRIES COMPRISES DANS 1 MLLIMÈTRE}

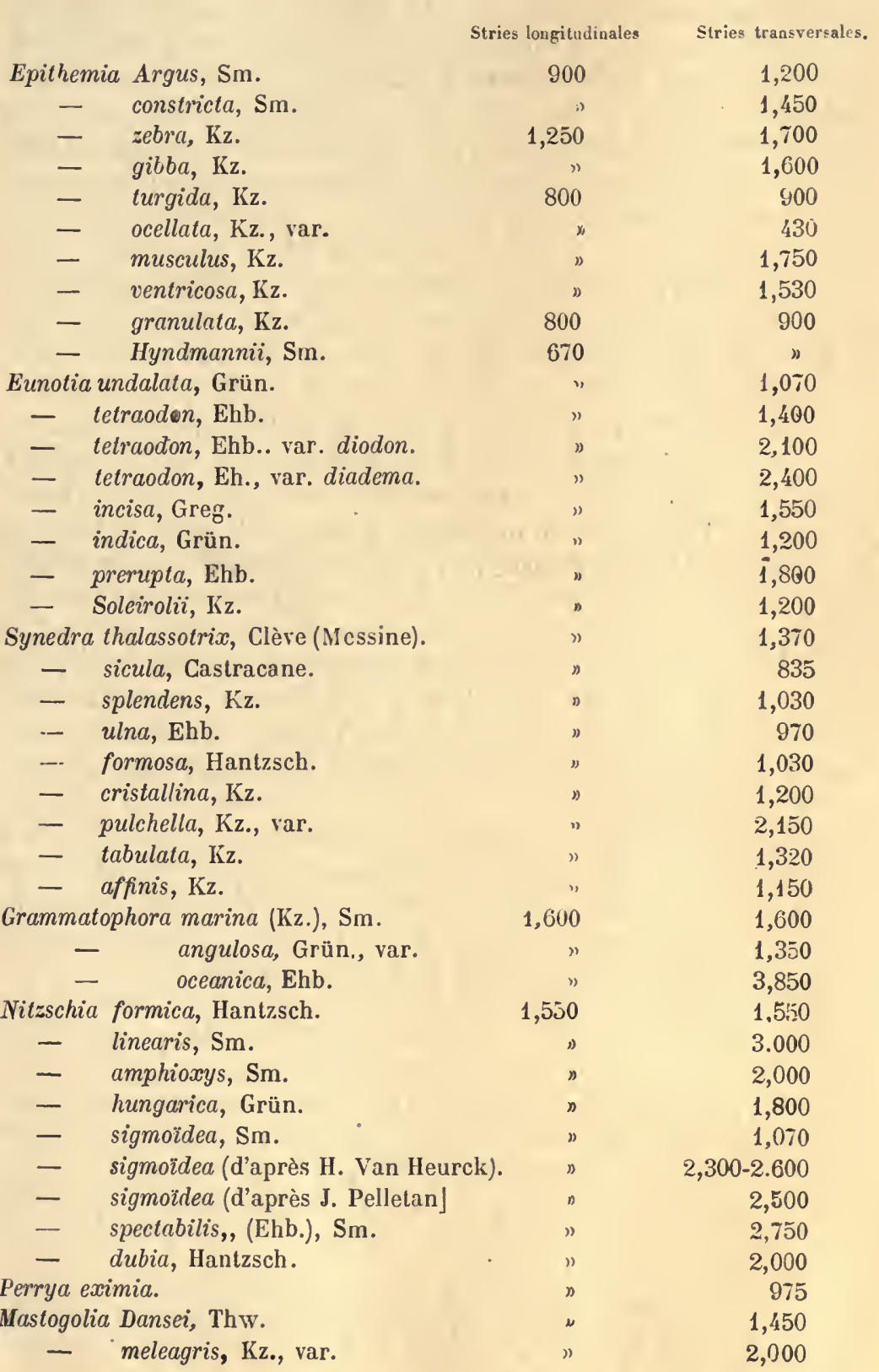


- Braunii, Grün. var.

- marginulata, Grün., var.

1,600

- exigua, Lew.

2,600

970

$9 \% 0$

- subsessilis, Kz.

- longipes, Ag.

brevipes, Ag.

Cymbosira Agardhii, $\mathrm{Kz}$.

Achnanthidium lanceolatum, Bréb.

Cymbella pisciculus, Ehb.

1,250

- heteropleura, Ehb.

de 900 à 1,000

- affinis, Kz., var.

- helvetica, Kz.

1,200

- scotica, Sm.

- kamtschatica, Grün.

de 1,100 à 1,300

1,200

900

- navicula, Ehb.

1,500

- cuspidata, Kz.

1,230

1,100

1,030

1,130

1,250

1,670

Rhoïcosphlcnia curvata, Grün.

Navicula ambigua, Ehb.

1,900

2,200

- serians, ${ }^{\prime} \mathbf{K z}$.

2,600

1,700

- sculpta, Ehb.

1,450

1,900

- limosa, $\mathrm{Kz}$.

1,900

-- quinquenodis, Grün.

- retusa, Breb.

650

- Reinhardtii, Grün.

900

1,050

1.600

- sphxrophora, Sm.

" (un peu irrég.) 1,500 amphisbæna, Kz.

- amphisbæna, Kz., var. permagna; Bailey. firma, $\mathrm{Kz}$.

1,550

- firma, var. affinis, Ehb.

- firma, var. amphirhyncus, Ehb.

1,900

1,570

- firma, var latissima, Ehb.

- firma, var. Hitskockii, Ehb.

- elegans, Sm.

1,650

1,650

- crassa, Greg.

- quadrata, Greg.

- entomon, Ehb.

- didyma, $\mathrm{Kz}$.

- elliptica, Kz. 


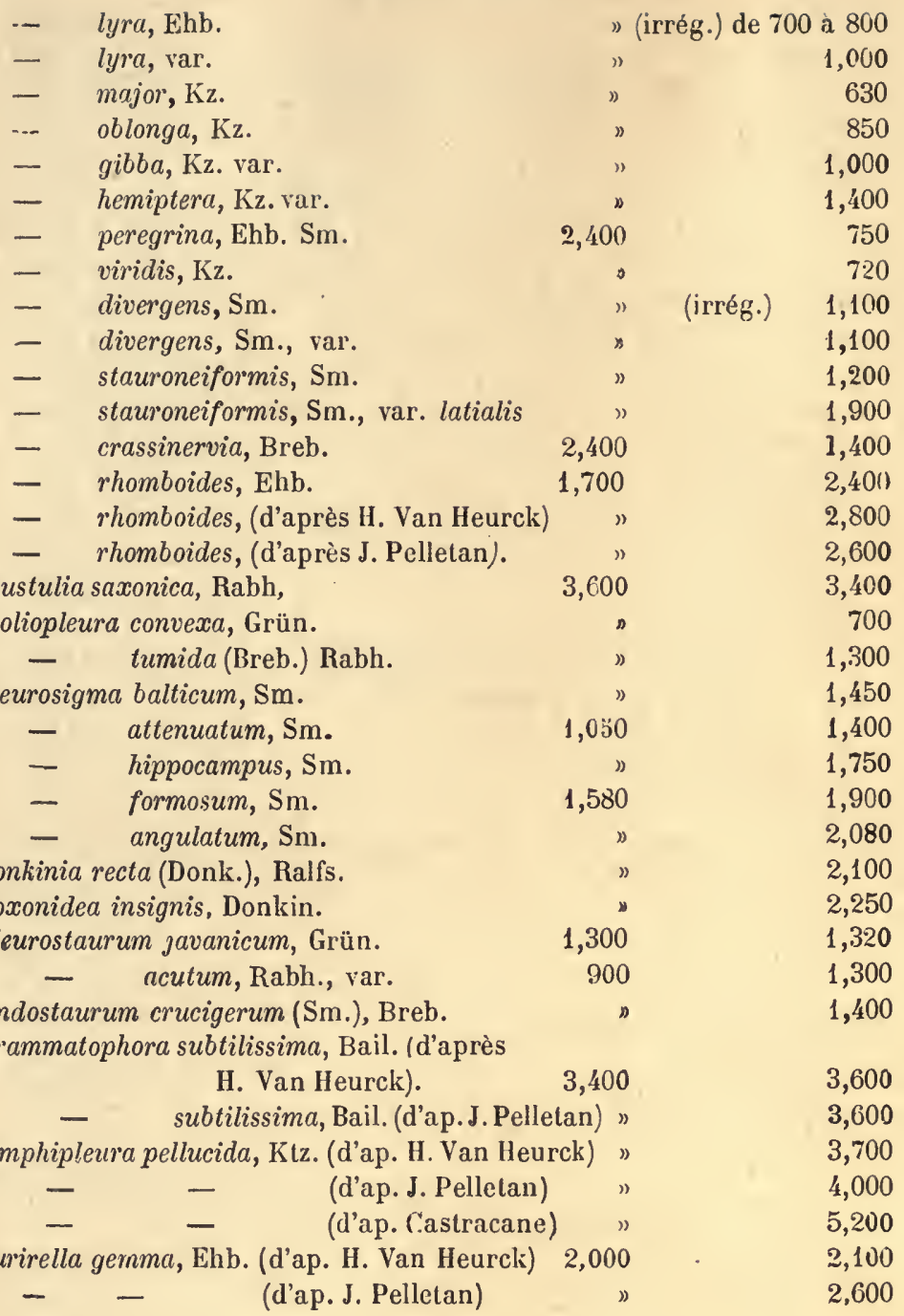

\section{§ 3. - Appendices ET dispositions particulières}

Outre les détails de structure, cỏtes, stries, perles, grains, yeux, etc., que présentent ordinairement les valves des Diatomées, on trouve souvent différents appendices, soit en dehors, soit en dedans de la valvè.

Nous pouvons, à la rigueur, classer parmi les appendices cerlaines ondulations de la surface de la valve qui produisent quelquefois comme des nervures longitudinales plus ou moins saillantes sur cette surface, 
nervures qui peuvent prendre une grande extension et constiluer ce qu'on appelle des ailes. Dans le premier cas, on dit que la valve est carénée, comme dans les Nitzschia; dans le second, qu'elle est ailée, comme dans les Surirella.

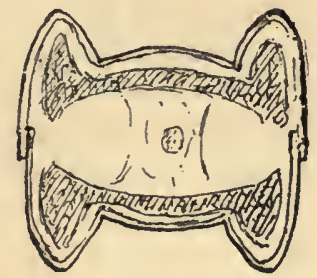

Fig. 60. Coupe transversale du Suritella ovalis.

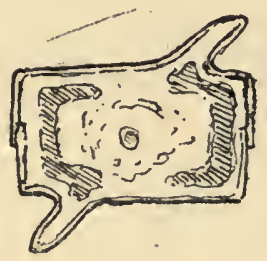

Fig. 61. Coupe transversale de l'Amphiprora baltica.

Il arrive aussi que les valves ont leur surface ondulée transversalement, ce que l'on reconnait facilement par les différences de mise au point quand on examine le frustule par la face valvaire, mais mieux encore quand on

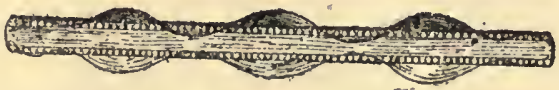

Fig. 62. Cymatopleura solea. (Face connective),

la regarde par la face connective. T'el est, par exemple, le Cymuıopleura solea. (Fig. 62).

Mais ce que l'on désigne particulièrement sous le nom d'appendices, ce

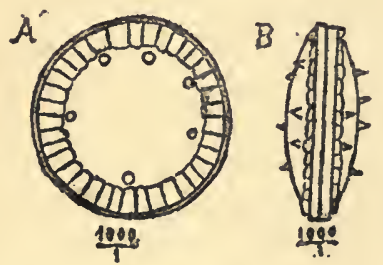

Fig. 63, Cyclotella sexpunctuta, J. Deb.

IIontrant les 6 appendices épineux auxquels cette espèce doit son nom.

sont des pointes, des épines, des cornicules, quelquefois fort longues, rameuses, qui se dressent en certains points de la valve. Ces productions paraissent souvent dues à un prolongement plus ou moins considérable, en 
dehors de la valve, des piliers intéralvéolaires, c'est-à-dire de ce que nous avons désigné sous le nom de "têtes de clous". On en voit des exemples

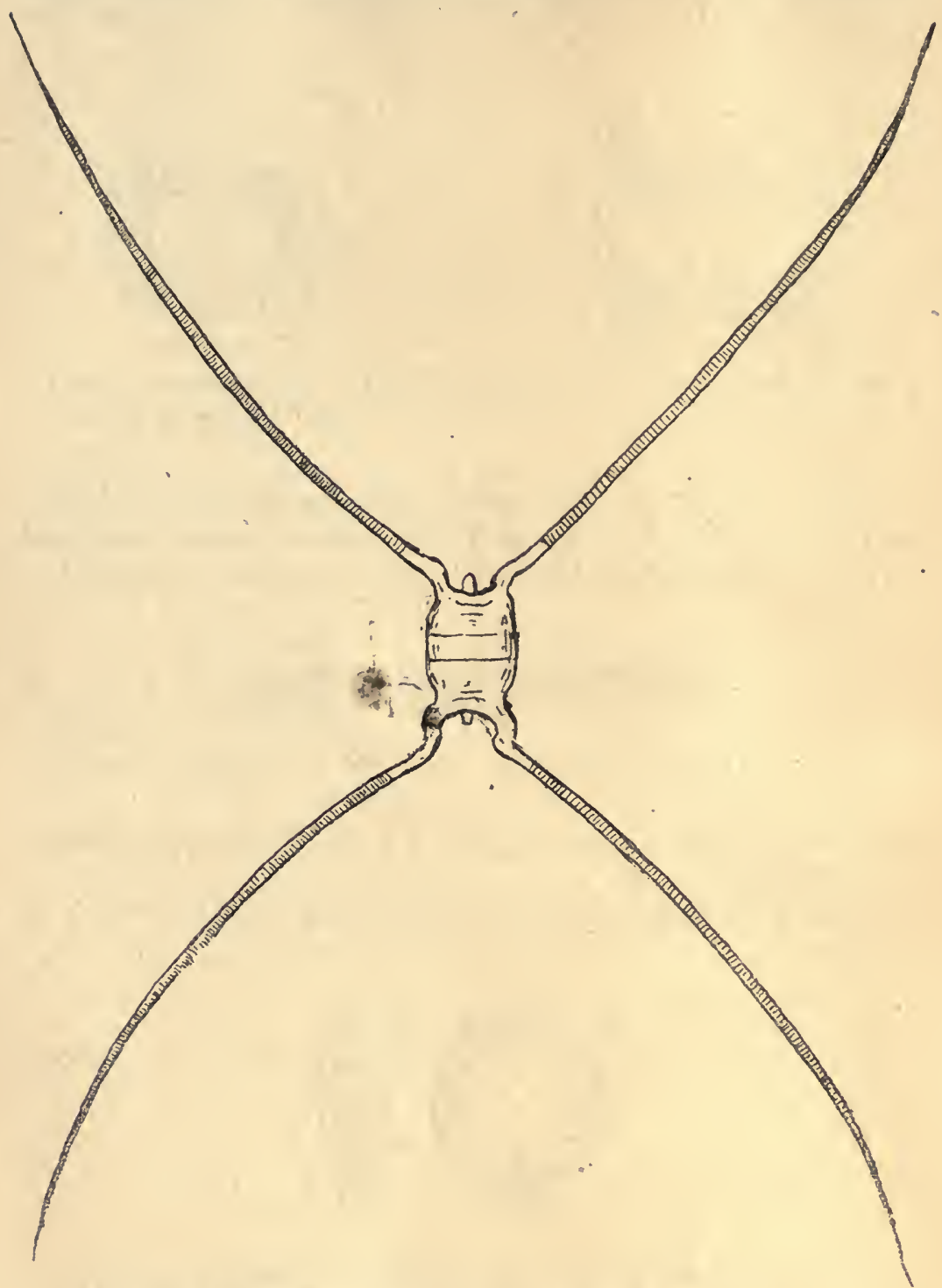

Fig. 64. Chøetoceros atlanticus. Cl. var. lumescens, Grün. (1).

dans le Cyclotella sexpunctata, dans le Stephanopyxis corona ou certains piliers, disposés en cercle sur une des valves, se développent en

(1) D'après M. H. Van Heurck, Syn. Diat. Belg. Pl, 83 ter. 
pointes égales et bifurquées formant une couronne, tandis que l'autre valve est hérissée comme un oursin.

D'autres fois, ces pointes, rui peuvent être tre̊s longues, ne sont que le dẻveloppement exagéré des extrémités de valves naturellement pointues, où d'aspérités de la valve en certains points disposés ordinairement d'une maniẻre très réguliẻre.

Ces prolongements donnent à certaines espéces, comme les Rhizosolenia, les Chætoceros, les Cylindrotheca, les Skeletonema, etc., les formes les plus caractéristiques et les plus singulières.

Nous signalerons encore, parmi les appendices, des productions des valves qui, au lieu de faire saillie à l'extérieur, font saillie à l'intérieur et forment des crêtes ou fausses cloisons pénétrant plus ou moins profondément dans la cellule. ()n les appelle viııx (au singulier : vitta). Mais

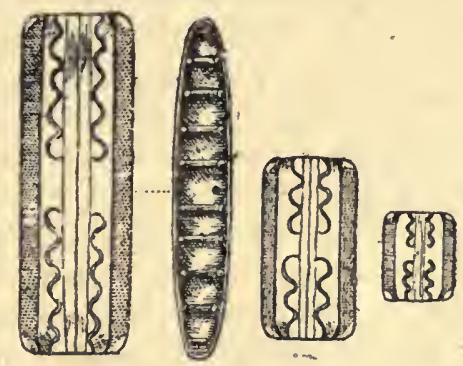

Fig. 65. Grammatophora marina.

quelquefois ce sont des cloisons complètes, sans doute perforées. On les appelle alors sepıa (singulier : septum).

Quand on examine à plat, par la face valvaire, les frustules qui présentent des cloisons ou des fausses cloisons, on voit celles-ci figurant comme des lignes épaissies, et l'on use peut se rendre compte facilement de leur profondeur qu'en examinant les frustules de trois-quarts, pour ainsi dire, en les faisant rouler dans la préparation.

Les lignes ondulées, figurant des serpents, que l'on voit sur les valves des Grammatophora, sont des fausses cloisons. On en voit encore de nombreuses dans les Rhıbdonema, les Tabellaria, les Striatella, etc. Nous en indiquerons de fréquents exemples et nous signalerons les autres détails particuliers de structure que présentent certaines Diatomées dans la description des principales espéces.

Dispositioxs spéciales. - Nous devons ajouter que si la surface des valves, chez un grand nombre d'espèces, présente des ailes, des carènes ou des ondulations, comme nous l'avons indiqué pour le Surirella ovalis, le Cymatopleura solea, des pointes plus ou moins saillantes, comme 
chez le Cyclotella sexpunctata et surtout les Stephanopyxis, et des appendices plus ou moins remarquables, les frustules affectent quelquefois une forme très singulière par suite de flexions ou même de torsions sur eux-mêmes.

Nous avons déjả signalé la double flexion en $\mathrm{S}$ qu'éprouvent les valves des Pleurosigma, de cerlains Nitzchia, comme les Pleurosigma angulatum, P. scalprum, Nitzschia sigmoidea, etc. ; mais outre

1

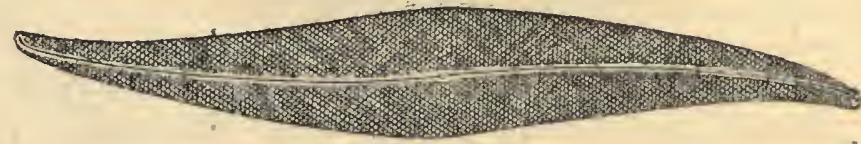

2

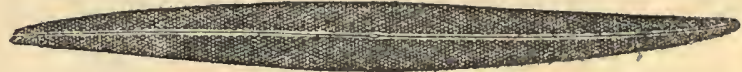

Fig. 63. - 1. Pleurosigma angulatum

2. Pleurosigma intermedium

cette flexion dans le plan général du frustule, plusieurs Diatomées s'infléchissent dans livers plans, et, comme on peut le prévoir, ce phénomène se produit surtout dans les espèces plus ou moins discoïdes, c'est-à-dire présentant une assez large surface valvaire. Non seulement les valves, au lieu de rester planes, (comme dans les Coscinodiscus, dont la forme peut être comparée à celle d'une boite de dragées), peuvent se renfler en chaudron ou en dé à coudre (Pyxidicula); non seulement l'une peut se

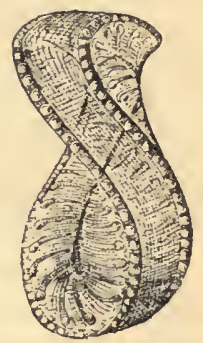

Fig. 67. Surirella spiralis.

renfler et l'autre rester à peu près plate, mais souvent elles se contournent de diverses façons.

Ainsi, dans les Campylodiscus, le frustule qui est å peu près circulaire comme contour, serait plat s'il était étalé sur un plan, mais il se replie suivant deux courbes, en forme de selle de cheval. Enfin dans la Surirella spiralis, le frustule, qui serait aussi à peu près plat, se tord en spirale autour de son grand axe (Fig. 67).

D'ailleurs, nous indiquerons, en décrivant les principales espèces, les formes, souvent étranges, qu'affecte le frustule de certaines Diatomées. 
Valves secondaines. - Outre les cloisons, souvent perforées, que l'on constate dans certains frustules, on trouve parfois, sous les valves, une seconde valve semblable, valve interne ou valve secondaire (Regenerationshülle). On en constate souvent l'existence lorsque les frustules se rompent, notamment pendant les manipulations nécessaires pour le montage en préparation.

Ces valves secondaires ne se trouvent jamais sous les valves trẻs jeunes, mais seulement sous les valves anciennes. Nous les considérons comme une sécrétion supplémentaire de la membrane de cellule, une superfétation dont le but est de remplacer la vieille valve en cas de destruction par usure ou fracture. La membrane cellulaire secrète, en effet, de la silice d'une manière constante, et la valve secondaire est formée par une couche de silice qui ne s'est pas soudée, au fur et à mesure de sa formation, à la face inférieure de la valve. De sorte que celle-ci peut, en certains cas, subir une exfoliation, un dédoublement en deux feuillets, dont le feuillet interne est la valve secondaire.

Cette production, et l'exfoliation ou le clivage naturel qui en résulte, ne se forment que chez les vieilles valves. C'est pourquoi, comme le fait remarquer M. J. Deby, les frustules, qui sont formés d'une jeune et d'une vieille valve, se séparent toujours en un nombre impair de valves. Ces valves secondaires sont d'abord aréolaires et perforées, mais au fur el à mesure que la silice les épaissit, les orifices se ferment. " Dans quelques cas, dit M. J. Deby, ces orifices se remplissent tout à fait d'une masse dense et saillante de silice douée d'un indice de rélraction plus élevé que celui de la substance propre du reste de la valve, de maniẻre à paraître comme des granules rouges ou roses sur un fond verdâtre avec les meilleurs objectifs à immersion. '

Noyau, double noyau, taghe germinative. - Immédiatement sous la carapace siliceuse est la membrane de cellule, membrane vivante qui sécrẻte la silice. Elle forme comme un sac qui enveloppe tout le contenu de la cellule. Dans les espêces qui se prêtent ả l'examen, on voit, dans l'intérieur' de ce sac, une bande centrale, sombre, ordinairement granuleuse, généralement appliquée à la face interne de la membrane cellulaire qui double les valves, et aussi le long des zones ; elle est contractile et extensible, et elle paraît diviser l'intérieur de la cellule en deux parties symétriques; sa surface forme des plis, quand on la regarde de côté, comme s'il y avait une fine membrane, plis qui dessinent comme des filaments.

D'après, M. H. L. Smith, dans les formes discoïdes, cette bande est tout à fait centrale ; sur la face valvaire elle mesure à peu près le quart du diamètre de la valve, et sur la face connective elle a la forme d'une bande resserrée au milieu, en sablier. On la voit bien sur les grands Navicula et les grands Surirella, quand l'endochrôme n'est pas trop foncé.

La substance qui forme cette bande est à peine colorée, mais le chloro- 
iodure de zinc, qui teint en vert pâle les autres matières intérieures, la colore en brun foncé. Les extrémités des frustules se colorent de même, comme si la substance en question était répandue partout sous les valves, mais plus épaisse seulement dans la bande centrale et aux extrémités. Quand on brûle les frustules, on voit encore la trace de cette bande, bien que toutes les autres matières intérieures soient détruites.

C'est dans cette bande centrale que sont placés les globules considérés comme élant de nature huileuse. lls s'y meuvent, allant et venant depuis une extrémité du frustule jus qu'au centre, sans jamais franchir, à ce qu'il semble, celte partie centrale où est situé le noyatu. Cet élément est quelquefois facilement visible, mais souvent fort obscur. Nous ne croyons pas qu'on ait jamais distingué, dans ce noyau, un nucléole bien évident. Autour du noyau, on voit souvent quelques doubles lignes rayonnantes, signalées déjà par Pfilzer.

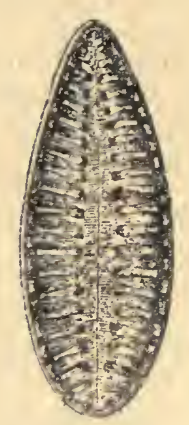

Fig. 68. - Surirella splendida. (F. minor).

Cette substance incolore répandue dans tout le frustule, condensée dans la partie centrale, et qui contient le noyau, substance qui brunit par l'iode, est, pour nous, le protoplasma cellulaire. C'est à la surface de ce sac protoplasmique qu'est répandu l'endochrôme, souvent disposé de telle sorte ou d'une coloration tellement intense que le noyau n'est pas visible. Mais, M. H. L. Smith a signalé, quant à ce dernier, des détails intéressants. Beaucoup d'espèces présentent d'une manière constante deux noyaux, même en dehors de la période de division. Tel est le Surirella splendida. Une espèce voisine, le Surirella elegans, n'en a jamais qu'un. - Dans la première, entre les deux noyaux et exactement au centre du frustule, on voit une petite tache sombre, que M. H. L. Smith appelle tache germinative "germinal dot ", tache qu'on observe aussi chez le Surirella elegans au-dessus du noyau unique, du côté de l'extrémité large du frustule.- C'est autour de cette tache, extrêmement plus petite que le noyau, que rayonnent des filaments protoplasmiques et des franges d'endochrôme. 
Cette " tache, » que l'éminent diatomiste américain n'a vue que dans ces deux espèces, nous rappelle la e tache embryonnaire , que M. Balbiani a signalée à côté du noyau dans certains ovules animaux. - Quant au douljle noyau, il peut provenir, suivant nous, d'un élat préparatoire, et plus ou moins prolongé, à la déduplication de la cellule. M. H. L. Smith l'a cons-

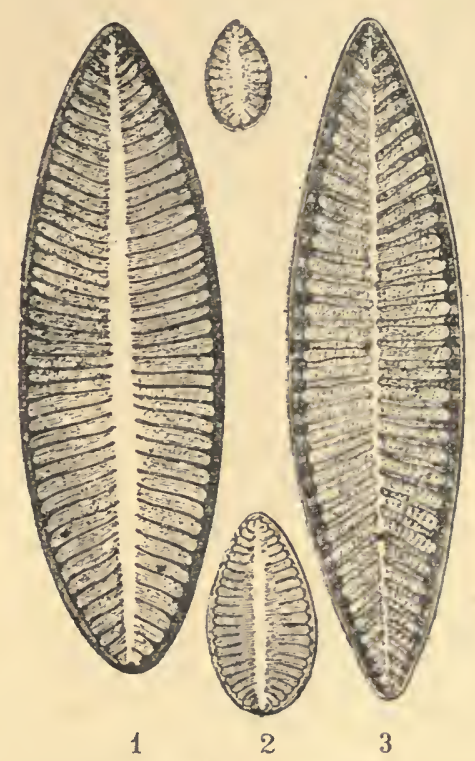

Fig. 69. - 1. Surrella elegans; $-{ }_{3}^{8}$ 2. S. ovata (2 formes). - 3. S. biseriata.

taté, en effet, sur le Cocconema lanceolatum, au premier printemps, sur "des? frustules qui venaient de "subir la déduplication, c'est à dire en pleine activité de reproduction fissipare, et qui vraisemblablement préparaient une nouvelle division. Du reste, les exemples de cellules multinucléées sont très fréquents dans les organismes inférieur's, et l'on trouve même des cellules bi-nucléées à l'état normal dans beaucoup de tissus animaux. 



\section{RECHERCHE ET RÉCOLTE DES DIATOMÉES}

\section{§ 1. - Les Diatomées dans la Nature.}

Les Diatomées existent dans toutes les eaux, les eaux douces, les eaux marines, les eaux saumâtres et même les eaux thermales, mais non dans les eaux putrides. Il est bien évident, toutefois, que ce n'est pas au milieu du courant des fleuves et des grandes étendues d'eau qu'il faut aller les chercher; c'est, souvent mèlées à des Desmidiées, sur les bords, les pierres, les plantes aquatiques, et tous les corps submergés, qu'on les trouve, formant un enduit plus ou moins mucilagineux, d'urıe couleur brunâtre ou d'un vert olive foncé. Cette couche brune se voit souvent à la surface de la vase dans les mares, les flaques abandonnées par les pluies, les fossés, au bord des élangs, sur les rochers des plages marines, les bois des estacades; souvent aussi, elle flotte à la surlace des eaux, dans les 'écumes soulevées par un dégagement de bulles de gaz.

Bien qu'on rencontre les Diatomées sur les objets baignés par les eaux dormantes, elles ne redoutent pas les courants les plus rapides, alors qu'elles trouvent un support solide. C'est ainsi qu'elles s'attachent aux pierres et aux bois qui forment la marge des barrages ou des cascades, el parlout, en un mot, où elles trouvent de l'eau ou une humidité suffisante, et un support ou un abri. Elles se fixent sur les algues, les conferves, toutes les plantes des rivages. Il en est mème qui vivent dans la mousse humide sur le tronc des arbres, les rochers el les murs, surtout à l'exposition du nord. On les a appelées Diatomées terrestres.

Comme, d'ailleurs, elles résistent fort longtemps à la dessication, on les trouve aussi sur les fonds des marais, des ornières, des fossés laissés à sec depuis plus ou moins longtemps. Elles forment, sur la vase à demi desséchée de ces fonds, la couche brune que nous avons indiquée et qui est caractéristique de leur présence.

Dans toutes ces localités, on les trouvera à l'état vivant, mais on les rencontrera aussi en grande quantité à l'état de frustule privé de matière organique, ou mème à l'état fossile, dans des gîsements qui s'étendent souvent sous des contrées tout entières. 
C'est ainsi que, les algues ou les animaux marins servant de nourriture aux oiseaux de mer, elles sont absorbées par ceux-ci avec les plantes sur lesquelles elles se font fixées ou avec les animaux qui les ont avalées. Comme leur carapace siliceuse est tout à fait insoluble dans les acides et dans les liquides gastriques, leur protoplasma, leur endochrôme, toutes les matières organiques qui entrent dans la constitution de leur cellule sont digérées par les oiseaux, mais les carapaces siliceuses se retrouvent dans les déjections. C'est dire qu'elles existent en grande abondance dans les guanos qui sont des amoncellements d'excréments laissés, depuis des siècles, par les oiseaux marins, sur certaines îles où ils ont pris, de génération en génération, l'habitude de se remiser.

Quant aux Diatomées fossiles, elles forment, comme nous l'avons dit, des gîsements considérables, des couches géologiques tout entiẻres. Les tripolis, dont on se sert pour le polissage des métaux, sont presqu'entièrement composés de leurs débris auxquels ils doivent la finesse et la dureté de leur grain. Le gîsement de cette nature le plus anciennement connu est, à ce que nous croyons, celui des marais tourbeux de Franzensbad, près d'Eger, en Bohême, dont la terre siliceuse a été reconnue par Fischer comme formée de frustules de Diatomées. Ehrenberg vérifia cette assertion et constata que ces frustules appartenaient à des Navicula viridis et N. major. Ce fut la première découverte de Diatomées fossiles. Bientôt, il fut reconnu que la terre siliceuse (Kieselguhr) de l'lle de France, la farine de montagne (Bergmehl) de Santa-Fiora, en Toscane, qu'on avait parfois mêlée à la farine ordinaire pour en faire du pain, étaient composées de frustules de Diatomées. L'immense couche de tripoli (Polirschiefer) de Bilin, en Bohême, exploitée sur une profondeur de 40 métres est formée de carapaces de Melosira, aussi bien que le dépôt de Planitz, en Saxe.

D’autres dépôts ont été trouvés à Lünebourg, à Ostie, à Oran, en Espagne, dans le Jütland, au Japon. En France, on a rencontré des gîsements composées d'espèces marines dans les terrains tertiaires. La détermination des espèces de Diatomées fossiles permet donc de préciser la nature des alluvions, et cette détermination est facile, car la plupart de ces espèces se trouvent encore vivantes de nos jours.

C'est ainsi que l'on retrouve "des preuves évidentes, dit M. J. Girard, du séjour des eaux dans des dépôts recouverts aujourd'hui d'épaisses couches de terre. Berlin repose sur une tourbe argileuse, de 7 à 20 métres de hauteur, composée de débris de Diatomées. Le lit inférieur de l'Elbe, jusqu'au dessous de Hambourg, est encombré de vases auxquelles sont mélangées des dépouilles organiques microscopiques. A Wismar (Mecklembourg-Schwerin), il se dépose par an 640 mètres cubes de corps siliceux. analogues aux Diatomées. En 1839, on a retiré du bassin du port de Swinmunde, à l'embouchure de l'0der, 90.000 mètres cubes de vase dont le tiers se composait d'organismes microscopiques ; ces êtres vivent 
dans tous les climats ; les limons des fleuves en charrient des milliards; les vases de la Mer Noire et du Bosphore contiennent jusqu'à 46 espéces déterminées par le micrographe Ehrenberg. On en a trouvé dans les eaux qui avoisinent les glaces du póle antarctique; les rivières et les marais salants de tous les pays en sont remplis. Dans la Géorgie (Amérique), dans la Floride, des vases diatomifères forment des bancs d'une étendue considérable. Des organismes microscopiques ont aussi été découverts dans le sens vertical, résultat probable du séjour des eaux à des époques préhistoriques. On signale les Diatomées par couches prodigieuses: la ville de Richmond (Virginie) est bâtie sur un lit de leurs débris, qui a 6 mẻtres d'épaisseur (Smith). Dans l'île de Mull (Écosse), le lac Boa, dont le fond desséché apparlient à la période jurassique, a fourni au professeur Gregory 130 espéces nouvelles (18ว̋4). "

Dans ces dernières années, on a trouvé un très grand nombre d'autres gisements fossiles parmi lesquels nous pouvons citer ceux de Santa Barbara et de Santa Monica en Californie, sans oublier ceux de Ceyssat et de Randanne, dans le Puy-de-Dôme, dont la terre siliceuse, contenant 87 pour 100 de débris de Diatomées, sert, sous le nom de randannite, à la fabrication de la dynamite (1). D'autres gîsements existent dans l'Ardêche, et l'on vient encore en Amérique, dans le Maryland, en forant un puits artésien, d'en découvrir un nouveau qui a fourni des espèces inconnues jusqu'à présent (?).

L'existence des Diatomées fossiles dans les couches profondes du sol devait laisser supposer qu'on en trouverait aussi dans les étages, plus profonds encore, formés des débris de la végétation qui couvrait la terre dans les temps anciens. En effel, M. Castracane, en incinérant de la houille et en recueillant les cendres, y a trouvé des Diatomées.

Enfin, si la vase des ruisseaux et des rivières renferme de ces algues, les fonds marins n'en sont pas dépourvus. Détachées des plantes marines qui vivent sur les côtes, emportées par les vagues, brassées par les courants et les marées, elles finissent par tomber lentement sur le fond des océans, quelquefois très loin de toute terre, et les dernières explorations sous-marines ont ramené dans leurs dragues, mèlés à des coquilles de Polycystines et de Foraminifères, des débris de Diatomées arrachés aux profondeurs de la haute mer où ils reposaient peut-être depuis des milliers d'années.

On voit donc que,pour les diatomistes, le champ des recherches est des plus élendus et qu'il lui faudra visiter non seulement les loealités oủ il pourra trouver les Diatomées dans leur site naturel et à l'état vivant, mais, quand l'occasion s'en présentera, les couches: profondes du sol et du sous-

(1) Les gisements siliceux fosssiles de l'Auvergne ont été étudiés par MM. Leuduger-Fortmorel et Paul Petit dans un travail qui a paru, en 1878, dans le Journal de Nicrographie, T. II, $\mathrm{n}^{\text {os }} 3$ et 4. 
sol, les matériaux des forages et des fouilles, la carène des navires, les pattes des ancres, les bois flottés, les plantes aquatiques rapportées des pays étrangers pour les besoins de lindustrie ou de la pharmacie, tous les objets en un mot, qui auront été, à un moment ou à un autre, en contact avec les eaux. Ce sont aulant de sources oủ le collectionneur pourra faire de riches récoltes d'espèces exoliques.

Le tube digestif des animaux marins, des oiseaux, des poissons, des mollusques, des annélides, de tous les ètres, enfin, qui se nourrissent de plantes sur lesquelles les Dialomées peuvent se fixer, ou qui vivent dans un milieu habité par elles, en contient ordinairement une grande quantilé, et la visile de l'estomac des huitres, des moules, des palourdes et de lous les mollusques qu'on trouve partout sur les marchés est, à ce point de vue, toujours très intéressante.

Nous donnons ci-dessous, à titre de renseignement, une lisle des principaux gìsements de Diatomées qui ont été découverts dans ces dernières années, non seulement dans l'intérieur des conlinents, mais dans les vases marines de cerlains golles et les dépôts formés à l'embouchure de cerlains fleuves.

\section{PRINGIPAUX GISEMENT'S, DÉPôtS OU LOCALITÉS DONT LES DIATOMÉES ONT ÉTÉ ÉTUdéEs (1).}

Aberdeen.

Afrique Australe.

Alsō-Esztergaly (Hongrie).

Agoa (Baie d').

Amazone (Emboucliure de l').

Amsterdam (Ile d').

Ardèche.

Auckland.

Australie.

Bail, Californie, (Et. Un.).

Bajtha (Hongrie).

Backer river (Afr. Centrale).

Baléares (lles).

Baltique (Mer).

Barbades.

Bartholomée (lle).

Battaglia.

Bencale.

Berkum.

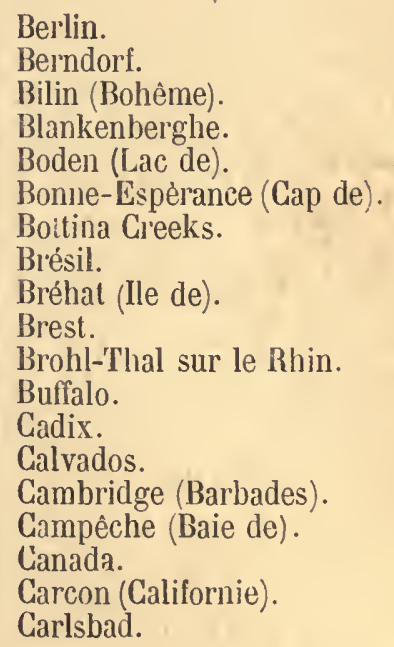

Berlin.

Berndorf.

Bilin (Bohême).

Blankenberghe.

Boden (Lac de).

Bonne-Espèrance (Cap de).

Bottina Creeks.

Brésil.

Bréhal (lle de).

Brest.

Brohl-Thal sur le Rhin.

Buffalo.

Cadix.

Calvados.

Cambridge (Barbades).

Campêche (Baie de).

Cianada.

Carcon (Californie).

Carlsbad.

(1) Nous n'avons pas la prétention de donner ici une liste complète, nous indiquons seulement les gisements les plus célèbres, dont on trouve des échantillons dans le commerce, ou les localités dont les Diatomées ont frit 'objet de publications spéciales. 
Caroline du Nord.

Carpentarie (Golle de).

Carrighill.

Carteret.

Caspienne (Mer).

Catanisella.

Ceyssat (Puy-de-Dôme).

Cebu (lles).

Chalk Munt (Barbades).

Cherbourg.

Cherrylield, Maine, (Elats-Unis).

Cheslu.

Clicago.

Clıristianstadt.

Côme (Lac de).

Constintinople.

Cornwallis (Nouvelle-Écosse).

Corse (lle de).

Cuba.

Cuxhaven.

Dalmatie.

Davis (Dérr. de).

Degarinas (Suède).

Delaware.

Demerara.

Dolgelly (Terrede).

Dolje (Hongrie).

Domblitten.

Dresde.

Drondjeim (Norw).

Duck Pond. Maine, (États-Unis).

Durrnberg.

East Stoughton, Massachusets (É. U.)

Ebstorf (Hanovre).

Eger (Boheme).

Elephant Point (Bengale).

Elesd (Hongrie).

Erié (Lac).

Exmouth.

Falaise.

Fall River, Orégon, (États -Unis).

Fano.

Falsö Esztergaly (Hongrie).

Finislère (Cap).

Finland.

Finmark.

Floride.

Fosarn (Iles).

Frahan.

Franzensbad (Bohême).

French's Pond, Maine (Élats-Unis).

Gallopagos (Iles).

Gérardiner (Lac de).

Gourvell.
Gottland (Ile).

Greal Salı Lake Deseret, Utah (É.-U.)

Habilsch wald.

Hacienda Escalera (Mexique).

Haminerlest, (Norwège).

Harris County, New Jersoy (E.-U).

Haslif ord.

Haverfordwest.

Heligoland (Ile).

Hildesheim.

Hjerkin (Norwège).

Hochsimmer.

Honduras.

Hong-Hong.

Hourdel.

Hulson River, New-York (É.-U.)

Hull.

Iclıaboë.

Ipswicl, Massachusets (États-Unis).

Isclia.

Isiria (Cap d').

Jan Meyen (Ile de).

Japon.

Java.

Jenissey.

Jérémie (Détroil de), Taïli.

Jone Valley.

Jonkoping.

Kärafjosd (Finmark).

Kamlschatka.

Kekko (Hongrie).

Kerguelen (lle de).

Kiel.

Kinord Loch.

Kinross.

Klamasch (Lac de).

Klicken sur Elbe.

Kilkel.

Korensmunster.

Krungjotrap.

Laconia, New Hampshlre (E.-U.)

Lamlash (Baie de).

Lara.

Larne Lough.

Lillhagjon.

Lima.

Liverpool.

Livourne.

Loka (Suéde).

Lough Mourne.

Lünebourg (Hanovre).

Lyel (Baie).

Magellan (Détroit de).

Mahé (Iles Seychelles. 
vanille.

Menat (Puy-de-Dỏme).

Mexico.

Mncar. .

Mogyorod (Hongrie),

Monmouth, Maine (Élats-Unis).

Monterey.

Monticello, New-York (Étals-Unis).

Moron (Espagne).

Mors (Ile) Jütland.

Mull, Lac Boa, (Écosse).

Nancoori.

Naparina.

Ngucy (Madagascar).

Nlagara Falls.

Nicobar (Iles).

Nimes.

Nimrod Sound (Chine).

Norfolk.

Norrland (Suède).

North Providence, Rhode Isl. (Ê-U.).

Noltingham, Maryland (Etats-Unis).

Nykjöbing (Julland).

Oamaru (Nouvelle-Zélande).

Oberhohe.

Oldenberg.

Oran.

Ormesby.

Ostende.

Ovalau.

Pabello do Pico.

Para (Rivière).

Pentsch (Silésie).

Pernambuco.

Peterburg, Virginie (Êtats-Unis).

Planitz (Saxe).

Plonchères.

Plymouth.

Premney.

Pudasjarvi (Finlande).

Puerto Rico (Antilles).

Quernero.

Quina, Massaclıuselts (Êtats-Unis).

Randanne (Puy-de-Dòme).

Rappahannock.

Regla (Mexique).

Rhin (Chute du).

Richmond, Virginie (États-Unis).

Richnond River, New Hamps. (E-U.)

Ringkjöbing (Jutland).

Rhodes (Ile de).

Roquelle (Embouchure de la).

Rouge-Cloître (Belgique).

Rouillat (Puy-de-Dôme).
Rovigno.

Saint-Paul (Mer du Sud).

Saint-Salurnin (Puy-de-Dôme).

Salem, Massach. (Elals-Unis).

Samoa (lles).

Sall-Andrea (Mexique).

San Diego, Calif. (Etats-Unis).

Santa Barbara.

Santa Fiora (Italie!.

Santa Monica, Calif. (Êtats-Unis).

Santos.

Savitajpel (Finlande).

Séville.

Seychelles (Iles).

Shasta, Calif. (Elats-Unis).

Sidmoulh.

Sierra Leone.

Sierra Nevada. Calif. (E.-U.)

Simbirsk (Russie).

Sodankyla (Laponie),

Sodërtelge (Suède).

South Yarra (Australie).

Spalato.

Spilzberg.

Staplis Ranch.

Stavanger (Norwège).

Stratlord Cliff.

Sumatra.

Swinmude sur l'Oder.

Szakal (Hongrie).

Szent Peter (Hongrie).

Tacarigna (Lac) Nouvelle-Zélande.

Taili.

Tamise (La) près Grenwich.

Tampa (Lac) Nouvelle-Zélande.

Tay (Rivière).

Teignmouth.

Tibre (Ostie).

Tillewilz (Sibérie).

'Tokay.

Tonga Tabou.

Toome Bridge.

Torbay.

Trieste.

Trouville.

Upsal.

Uiah.

Valparaiso.

Venise

Vera Cruz.

Vienne.

Vierges (Iles).

Vocklabruk.

Vogelsgebirge. 
Vosges.

Wankarema (Cap).

Wedel (Holstein).

Wernamo (Suède).

Westerbolten.

Wismar (Mecklemb. Schwerin).

Yucatan.

Zell (Lac de).

Zurich.
Guanos de Bolivie.

- de Cillifornie.

- de l'ile Baker.

- des Iles Mexillones.

- du Méxique.

- du Palagonie.

- du Pérou.

- de Saldanha-Bay.

\section{§ 2. - RÉCOLTE DES DIATOMÉES}

La récolte des Diatomées n'exịge pas un matériel bien considérable ni bien gênant : quelques flacons et quelques tubes fermés avec un bouchon de liège, quelques morceaux de grosse toile, imperméable si possible, ou même des carrés de fort papier; une sorte de cuiller en étain, en fer ou

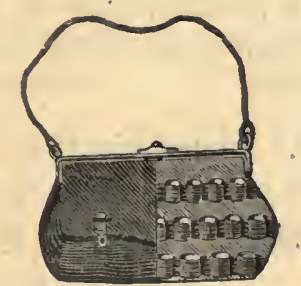

Fig. 70. Sac de M. Paul Petit, Pour la récolte des Diatomées.

en cuivre, permettant d'enlever délicatement la couche diatomitère qui recouvre la vase, et de râcler les pierres, les rochers, les bois submergés sur lesquels les Diatomées peuvent être fixées; une canne au bout de laquelle peut se visser un crochet pour attirer à soi les plantes trop éloignées ; une bonne loupe ou, si l'on veut, un petit microscope de poche; quelques lames de verre ou de mica, - et c'est tout.

Le flacon et les tubes peuvent être trẻs commodément disposés dans un sac de voyage, comme l'a indiqué M. Paul Petit. On peut de cette manière en transporter aisément un grand nombre, placés sur plusieurs rangées : au fond, sont arrimés les plus grands flacons, et dans la rangée supérieure les tubes (Fig. 70)

Au surplus, comme on le comprend, chacun peut disposer comme il l'eitend un appareil de transport, commode et léger, dans lejuel les récipients ne courent pas le risque d'ètre brisés.

Les morceaux de toile imperméabie ou de gros papier servent à envelopper les paquets d'Algues ou de plantes aquatiques sur lesquelles on a 
reconn'x la présence des Diatomées et qui n'ont pas pu trouver place dans les flacons ou daus les tubes, ces deriniers étant destinés surtout à placer ce qu'on appelle des récoltes pures, c'est-à-dire des amas plus ou moins considérables formés, en grande partie au moins, par une seule et même espèce.

Le diatomiste expérimenté est, en effet, renseigné le plus souvent sur la nature de la récolte qu'il fait dans telle ou telle localité, dans tel ou tel

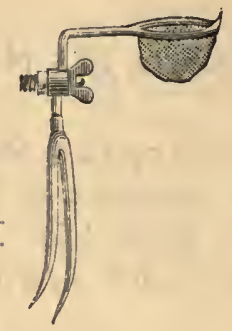

Fig. 71. Filet à crochet bi dent de M. Paul Petit, se vissant au bout d'une canne.

site, soit par l'aspect même des matérịaux, soit par leur' couleur, soit par leur forme. Dans tous les cas, il est toujours utile qu'il puisse, à l'aide d'une forte loupe ou d'un petit microscope de poche, se rendre compte des espèces qu'il recucille.

Comme loupe, il peut employer la loupe Codurington, ou mieux encore la loupe de Brücke. Plusieurs opticiens construisent aussi de pelits instru-

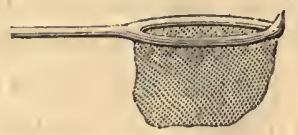

Fig. 72. Filet à lame tranchaute, de M. Giraudy.

ments à giossissement variable qu'on appelle diatomescopes et dont: l'usage est commode. Il existe aussi plusieurs mollèles de pelils microscopes composés, très porialifs et que l'on peut aisément employer dans le.s. herborisations. Nous reviendrons plus tard sur ces instruments.

Le diatomiste recherchera donc les plantes aquatiques, ces conferves molles et comme glaireuses qui garnissent le bord des rivières, des: ruisseaux, des mares. ll pourra atleindre les plantes placées hors de sa. portée à l'aide du crochet monté au bout d'une canne. M. Paul Petit conseille, da!!s ce but, un instrumenl composé d'un double crochel surmonté , 
d'une petile drague en fil de laiton, instrument qui peut ainsi servir à deux fins et permet de ramener dans le fiiet les plantes que l'on a arrachées avec le crochet. On peut aussi employer utilement le filet de M. Giraudy, filet en laiton, monté sur un petit całre métallique, dont la partie antérieure se relève en lame tranchante. Cet instrument se visse, comme le précédent, à l'extrêmité d'une canne. It est inutile d'ajouter que l'un et l'autre peuvent servir dans les herborisations pour recueillir non seulement les plantes aquatiques, mais toutes celles qui sont hors de portée.

Il y aura ensuite à récolter les enduit; brunâtres déposés sur la vase des marais, des ornières, des flaques d'eau. Ce travail se fait facilement à l'aide de la cuiller d'étain ou de laiton. Il faudra enlever ces croùtes, d'une main légère, en prenant en mème temps le moins possible de la vase sous jacente. Ces enduits seront déposés dans les tubes ou dans les pelits flacons avec un peu d'eau, pour les empécher de se dessécher.

On grattera de mème l'enduit sur les parois des citernes, sur les constructions submergées, les pierres, les rochers, enduits qui les rendent quelquefois si glissants et comme glaireux. Il sera souvent plus commode, dans ce cas, de substituer à la cuiller une petite plaque de métal, en cuivre ou en zinc, tranchante sur son bord antérieur.

Quant aux masses floconneuses nageant à la surface de l'eau, la cuiller, emmanchée, au besoin, au bout de la canne, ou les petites dragues dont nous avons parlé, permettront aisément de les recueillir.

Les espèces pédicellées ou filamenteuses peuvent être reconnues très souvent à l'aspect qu'elles donnent aux colps qu'elles recouvrent, à leur consistance particulièrement glaireuse, en raison de la matière Inucilagineuse qui forme leur pédicelle, leur tube ou leur thalame. Souvent, elles donnent aux plantes un aspect velouté ou irisé dù à l'accumulation de tous les pédicelles qui font comıne des villosités microscopiques dont la transparence produit des jeux de lumière.

Si l'on veut conserver ces espèces dans leur intégrité, ménager les pédicelles et les tubes, qui présentent des caractères pour la classification, mais sont souvent très fragiles, on comprend qu'on devra les traiter avec les plus grandes précautions. On arrive presque toujours, en déposant dans un flacon, avec un peiı d'eau, la plante qui les supporte, à retrouver intacts, en rentrant chez soi, un certain nombre d'individus; cependant, les filaments sont queliuefois tellement fragiles, ou les échantillons que l'on rencontre si peu nombreux, qu'il peut y avoir avantage ả leur faire subir sur place une prévaralion conservalrice. C'est dans ce but que Brébisson, M. Guinard et nombre de diatomistes. ont conseillé l'emploi de petites lames de mica. Ces lames de mica ont la furme d'un carré de 1 centimètre

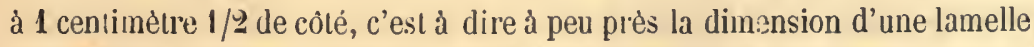
couvre-objet ordinaire. Voici, ce que M. Guinard dit à ce sujet :

"Lorsqu'au moyen du microscope, vous reconnaissez que vous avez rencontré une Diatomée pédicellée et dont le pédicelle est excessivement 
fragile, par exemple ies Cocconema, les Rhipidophora, etc., il est de toute nécessité de rapporter ces échantillons dans leur intégrilé la plus parfaite ; c'est alors que l'on a recours aux pelits carrés de mica. "

"Vous délachez avec de pelites pinces les fragments de mucosité brunâtre que présente une agglomáration de ces espéces; vous les étendez avec précaution sur vos micas, en y joignant une petite goutle d'eau. Par ce mode de préparation et d'examen préalable, vous évitez l'erreur dans laquelle sont tombés plusieurs algologues qui ont considéré comme dépourvues de pédicelle cerlaines espèces qui en sont munies, et qui, mises dans des flacons, sans contrôle, au moment de la récolte, avaient été privés de cet appendice par les secousses du voyage. A défaut de micas, vous pourriez aussi vous servir de petits carrés de papier buvard; mais le premier moyen est prélérable sous tous les rapports. "

"La rigidité et la transparence des micas, se prètent à un examen ultérieur sous le microscope, et de plus, sous celle forme, ils peuvent, sans qu'il soit besoin d'y revenir, être conselvée en lierbier. »

* Comme l'on n'a pas le loisir d'attendre que ces préparations extemporanées soient arrivées en un élat de siccité parfaite pour pouvoir les empaqueter, M. de Brébisson avait imaginé un pelit appareil très propre à cet usage. Nous allons donner, d'aprẻs cet auteur, la manière de le construire.

«Pile diatomique : Ce petit appareil est formé de disques de liège ou " simplement de bouchons coupés en rondelles d'une ou deux lignes - d'épaisseur, et enfilés par une tige de laiton reployée aux deux extré" milés, de manière à former un pelit bàlon d'une longueur convenable " pour être placé diagonalement dans un coinpartiment réservé à l'un des

" bouts de la boite destinée aux herborisations, ou, avec effort dans l'inté-

" rieur du chapeau de l'exploraleur algologiste. Les rondelles de liège sont

" pressées les unes contre les autres par un bout de ressort à boudin placé

- au milieu de la pile et s'enroulant librement autour de la tige centrale.

" On engage dans les fentes qui existent entre les rondelles un coin de la " lame de mica sur laquelle on a préparé l'espèce délicate. On peut ainsi en " placer un grand nombre qui se dessèchent sans se toucher. Un morceau " de liège entamé par des triits de scie peut remplacer la pile, mais avec " moins d'avantages (1)."

Pour terminer ce ıui a rapport à la récolte des. Diatomées nous rappellerons que l'on trouvera souvent des espèces intéressantes dans les dépôts d'alluvion, sur les objels qui ont été en contact avec des milieux diatomifères, les co fues de navire, les ancres, diver's oljjets provenant des pays étrangers. M. de Brébisson a trouvé une riche collection de Dialomées sur ce fucus connu dans les pharmacies sous le nom de mousse de Corse (Sphærococcus helminthocorton), M. P. Petit sur la mousse d'Irlande

(1) A. DE BrÉBissox, lettre à Ch. Chevalier. 
ou Caragahen (Chondrus crispus). Il y a sur toutes les plantes analogues d'aboudantes récoltes à faire. Rappelons encore qu'on en trouvera dans l'estomac des anin:aux marins ou aquatiques, particulièrement des illollusques, sur les coquilles de ces mèmes Mollusques, sur les carapaces des Crustacés, elc.

Il est inutile d'ajouter que les recherches devront être faites dans les eaux douces, l'eau de mer, les élangs saumâtres, et que la matière glaircuse de certaines eaux thermales renferme plusieurs espèces de ces pelites plan es.

Enfin, comme beancoup de Diatomées vivent libres, que certaines mèmes sont mobiles et, pour ainsi dire, nageuses, que d'autres sont souvent arrachées de leur support par le mouvement des eaux, en filtrant une quantité suffisante de certaines eaux, on pourra trouver sur le filtre des Diatomées en plus ou moins grand nombre.

Pour les Diatomées pélagiques, voici comment M. J. Brun recommande de les récolter et de les conserver.

" Quant aux organisınes pélagiques, on les prend en promenant un voile de soie au traveres des couches supérieures de l'eau. L'on récolte ainsi les espéces à l'état vivant, et elles ne sont guẻre mèlées qu'avec: un peu de poussières minérales. Le voile de soie doit être à mailles trẻs fines et tendu sur un cadre (ou un cercle) tenu verticalement, de manière à ce que la partie supérieure effleure la surface aqueuse. Ceci pendant que, soit le bras, soit le bateau, avance très lentement : Toutes ces espèces pélagiques restent fixées au voile sous forme d'une couche à aspect glaireux. Elles s'enlèvent de temps en temps au moyen d'une lame en corne souple et mince. La meilleure manière de conserver longtemps ces récoltes consiste à les mettre immédiatement dans une solution au quart d'acétate de potasse neutre. L'alcool que l'on emploie habituellement a l'inconvénient de contracier et de déformer la plupart de ces animaux ou végétaux inférieurs. L'acétate n'améne aucune déformation; i! arrête toute putrélaction et s'élimine facilement par un simple lavage à l'eau, lorsqu'on veut entreprendre l'étude microscopique (1). "

Nous ne pousserons pas plus loin ces considérations, certain que pour la recherche des Diatomées dans leurs divers habitats, leur récolte et leur conservation jusqu'au moment de l'étude, chacun trouvera, dans son esprit, les ressources nécessaires pour réaliser les dispositions et les procédés les plus convenables. Tous ceux qui ont herborisé ne seront jamais emlarrassés pour mener à bien ces petites opérations qui, souvent délicates, ne sont pas difficiles et n'exigent que des soins, de l'attention et de la patience.

(1) J. Brun. - Journ. de Micrographie, T. XI, 1887, n०5. - Ce procédé de récolte s'applique non seulement aux Diatomées pélagiques, mais aux Polycystines, Foraminifères et autres Rhizopodes. 


\section{V \\ TECHNIQUE DES DIATOMÉES}

\section{§ 1. - NETTOYAGE DES DIATOMÉES}

Après que les Diatomées ont été récoltées, la première opération à leur faire subir consiste à les séparer des sédiments sur lesquels elles reposent et à les détaeher des plantes auxquelles elles sont attachées.

Pour eela, on vide les tubes et les flacons dans des assiettes, des soueoupes ou des verres de montre, suivant la quantité de matière sur laquelle on opère, et, après avoir enlevé les eorps étrangers les plus volumineux, on ajoute un peu d'eau pure de manière à reeouvrir le dépôt; puis, on abandonne le tout, pendant 12 à 24 heures, dans un endroit fortement éelairé, mais non au soleil. Les Diatomées, qui sont vivantes, quittent la vase et viennent se déposer à la surfaee, ou mème sur les bords de l'assiette. On peut reeueillir avee un petit pineeau eelles qui sont venues se rassembler ainsi sur les bords, en une eouche légère plus ou moins brune, et on lave le pinceau dans un peu d'eau distillée. Ensuite, on déeante doueement l'eau qui reeouvre le sédiment déposé sur le fond de la soueoupe, et on enlève la eouehe supérieure diatomifère de ce sédiment, aree un pineeau, si elle est mince, aree une lame fine et tranehante si elle est plus épaisse. On trempe pinceau et lame dans un peu d'eau distillée dans laquelle les Diatomées se répandent.

Brébisson reeommandait deux proeédés partieuliers pour séparer les Diatomées du sédiment sans emporter avec elles une partie de la vase plus ou moins meuble dans laquelle elles sont comprises.

Déposant le sédiment diatomifère dans une soueoupe ou un verre de montre, suivant la quantité, on le reeouvre d'une rondelle de papier buvard qu'on y applique avee le bout du doigt, sans eomprimer eependant, et en évitant d'emprisonner des bulles d'air qui sépareraient la faee inférieure du papier de la faee supérieure de la vase. On laisse les ehoses dans l'état, pendant au moins 24 heures, à la lumière. $\mathrm{Au}$ bout de ee temps, on lève avec précaution la rondelle 
de papier et on la trouve couverte par dessous de Diatomées qui sont renues s'y attacher. On n'a plus alors qu'à laver le papier dans un peu d'eau pure pour avoir une récolte très propre.

Ou bien, on applique sur le dépôt une rondelle de linge un peu clair, toile ou mousseline, et on maintient le contact en posant par dessus le linge un anneau de métal d'un diamètre un peu plus petit que celui de la soucoupe. Au bout d'un ou deux jours, les Diatomées se sont séparées du dépôt et, cherchant la lumière et l'air, se sont déposées sur le linge dont elles ont traversé les mailles.

Si l'on a emporté, dans la récolte, des pierres, des morceaux de bois, des fragments de plantes garnis de Diatomées, après les avoir placés arec un peu d'eau dans une soucoupe, on les lave avec le bout du doigt pour détacher les Diatomées, qui se répandent dans l'eau avec quelques débris; ou bien on gratte les objets avec un canif ou un grattoir.

On opérera de mème avec les algues et les plantes aquatiques chargées de Diatomées, et lorsqu'elles présentent quelque résistance, on obtient en suspension une grande quantité de Diatomées arec très peu de sédiments et de débris. Quand il s'agit de plantes très molles, et qu'il n'est guère possible de laver ou de gratter, on les réduit, arec le doigt ou une spatule, en une pulpe aussi homogène que possible, et l'on traite cette pulpe comme nous l'avons indiqué plus haut pour les vases. C'est dans ce cas surtout que l'on obtient de bons résultats du procédé de la rondelle de papier ou de linge. On peut, d'ailleurs, renouveler l'opération plusieurs fois, et autant que l'on troure des frustules fixés à la rondelle. D'autres fois, on est obligé de détruire complètement les plantes par les agents chimiques, comme nous le verrons plus loin, pour parvenir à isoler les Diatomées.

Enfin, on peut conserver très longtemps les récoltes telles qu'on les a recueillies, en les laissant dans un endroit éclairé et en ayant soin d'ajouter de temps en temps un peu d'eau. Il arrive même ordinairement qu'en gardant ainsi pendant quelques jours les récoltes, les Diatomées se séparent peu à peu, et comme elles continuent le plus souvent à vivre, elles reprennent petit à petit leurs positions normales, s'orientent pour ainsi dire, se multiplient même, revenant aux conditions ordinaires de leur existence, et l'on en obtient de véritables cultures.

Les procédés que nous venons d'indiquer ont; en effet, pour résultat d'isoler', autant que possible, les Diatomées à l'état vivant. C'est dans cet état qu'on pourra les étudier au point de vue de leur histoire naturelle, de leur classification méthodique et des phénomènes biologiques qu'elles présentent. Cette étude a été jusqu'ici trop négligée, comme l'a très justement fait remarquer M. Paul Petit. 
On s'est trop borné à l'examen des frustules morts, du squelette, pour ainsi dire, de la plante, au préjudice de la plante elle-mème, qui, cependant, présente tant d'intérêt.

A cet état, on peut étudier la composition de la Diatomée, son protoplasma, son noyau, son endochròme, les diverses substances qu'clle contient; - et, en particulier, c'est sur la disposition de l'endochrôme que le professeur Pfitzer et M. Paul Petit ont fondé la première classifieation naturelle qui ait été faite de ces organismes. C'est à notre avis, une étude féeonde, à laquelle les diatomistes ne sauraient trop s'attacher et grâce à laquelle seule on parviendra à étendre les connaissances si incomplètes encore que nous avons sur les Diatomées considérées comme des ètres vivants et non plus seulement comme des petites lames de silice merveillcusement sculptées.

Mais ce n'est pas à cet état, pour ainsi dire, naturel, et dans lequel il serait d'ailleurs impossible de distinguer les détails du frustule, que l'on conserve les Diatomées pour les collections et qu'on les prépare pour l'observation. Il faut les dépouiller de leur protoplasma, de leur endochròme et de toute la matière organique qu'elles eontiennent ou dont elles peuvent ètre enveloppées, pour conserver uniquement leur carapace siliceuse.

Celle-ci, en raison mème de la matière qui la compose, et qui est de la silice pure, est complètement inattaquable par les acides autres que l'acide fluorhydrique et par les solutions mème assez coneentrées d'alcalis caustiques. C'est sur cettc propriété et eette résistance aux agents chimiques, alors que la matière organique est détruite par ces réactifs, que sont fondés les procédés de nettoyage des Diatomées.

Cependant, si les espèces d'eau douee sont munies d'une enveloppe solide très fortement silicifiée, il n' en est pas toujours de même de certaines espèces marines telles que le Pleurosigma angulatum, l'une des plus employées comme test, et plusicur's autres dont le revètement siliceux est beaucoup moins épais et moins résistant. Celles-ci devront done être traitées avec plus de ménagement.

Les procédés employés pour détruire la matière organique des Diatomées sont, d'ailleurs, analogues à ecux dont se servent les chimistes pour détruire la matière organique des substances dans lesquelles ils veulent, par l'analyse, rechereher des matières minérales.

\section{Traitement par l'acide nitrique.}

C'est le procẻdé le plus simple et le plus souvent employé pour' les Diatomées d'eau douce récemment récoltées. Voici comment on l'applique : 
Une certaine quantité du dépôt qui se forme dans l'eau où l'on a porté les Diatomées après les avoir séparées de la vase ou des objets sur lesquels elles étaient adhérentes, comme nous l'avons indiqué plus haut, cst mise dans un tube à essai', et, si l'on suppose qu'elles conticnnent encore quelques corps étrangers, des grains de sablc, par exemple, on ajoute par-dessus une certaine quantité d'eau. Bouchant alors le tube avec le pouce, on le secoue, de manière à mettre toute la masse en suspension dans lc liquide. Aussitòt quc l'agitation a cessé, les grains de sable, qui sont lourds, tombent tout de suite au fond du tube, tandis que les Diatomées, plus lćgères, restent plus longtemps en suspension. On décante alors rapidement dans un autre tube lc liquide, qui contient les Diatomécs en suspension, et on laisse au fond du premier tube le dépòt, qui est formé des grains de sable et autres corps lourds.

On laisse alors reposer pendant plusieurs heures ce second tube, pour donner aux Diatomécs le temps de se déposer à lcur tour. Quand l'cau est devenue parfaitemcnt limpide, que, regardée par transparence, elle ne laisse voir aucun corpuscule miroitant en suspension, on la décante de nouveau, dc manière à ne conserver que le dépôt avec une très petite quantité d'eau.

On versc par-dessus assez d'acide nitrique ordinaire pour recouvrir les Diatomécs de 3 ou 4 centimètres d'acide.

S'il s'agit d'espèces peu silicifiées, on laisse la réaction s'opérer à froid, mais pour celles dont la carapace est plus résistante, comme presquc toutes les Diatomées d'eau douce, il est nécessaire de chauffer. On prend alors le tube avec une pince en bois comme celles dont se servent les chimistes, ou avec une de ces pinces cn fer qu'on appelle fers à tuyauter et dont on a cnfilé chacun des mors dans un bouchon portant une cncoche du còté interne pour bien saisir le tube, et on porte celui-ci dans la flamme d'une lampe à alcool. Il faut tenir le tubc incliné pour diminuer la hauteur de la colonne liquide au-dessus du fond, empêcher les soubresauts et éviter de respircr les vapeurs acides qui se dégagent. On doit donc opérer à l'air ou dans la cage vitréc d'un laboratoirc, et dans tous les cas, dans une pièce où l'on n'a laissé aucun instrument en cuivre, microscope ou autrc.

On chauffe jusqu'à ébullition et l'on fait bouillir pendant une dcmi-minute à deux minutes, suivant la nature des espèces. Puis, on laisse rcfroidir. On ajoute alors de l'eau et on laisse le tout reposer pour permettre aux Diatomées de gagner lc fond.

Quand le dépôt s'est opéré, on décante avec précaution le liquide surnageant et on ajoute encore de l'eau pour laver les Diatomées et les débarrasser de l'acide qui les imprègne. 
On laisse encore reposer, puis on décante, et on ajoute de nouvelle eau, et ainsi de suite jusqu'à ce que l'eau décantée ne manifeste plus de réaction acide et ne rougisse plus le papier de tournesol. Il faut pour cela un assez grand nombre de lavages successifs et l'opération est toujours assez longue.

Enfin, pour enlever les dernières traces d'acide, on ajoute un peu d'ammoniaque que l'on décante encore, et on lave plusieurs fois à l'eau distillée.

On peut alors, après une dernière décantation, verser sur les Diatomées de l'alcool dans lequel elles se conserveront indéfiniment pour ètre montées au moment du besoin. M. J. Brun remplace l'alcool par une solution d'acétate neutre de potasse.

Toutes les Diatomées peuvent être traitées ainsi ; il faut néanmoins examiner au microscope une parcelle du dépòt, pour reconnaitre si elles sont bien débarrassées de toute la matière organique. Si les frustules n'étaient pas complètement nets, il faudrait recommencer.

Les Diatomées marines devront ètre préalablement lavées une ou deux fois à l'eau distillée pour les débarrasser du chlorure de sodium dont elles peuvent être imprégnées.

Dans tous les cas, il faut, pour ces opérations, se servir 'd'eau distillée (1), parce que celle-ci a la propriété de tenir en suspension les matières argileuses qui sont ainsi enlevées par décantation, tandis que dans l'eau calcaire ou saline, elles se déposeraient avec les Diatomées. Cette précaution est indispensable pour le traitement des Diatomées des estuaires marins (Van Heurck).

\section{Procédé par l'acide sulfurique et le chlorate de potasse.}

Il arrive souvent que les Diatomées contiennent, soit leur appartenant en propre, soit à l'état de matière étrangère, une telle quantité de substances organiques que l'acide nitrique, mème bouillant, ne suffit plus pour brûler et détruire toutes ces substances; il faut alors employer des matières comburantes plus énergiques, et par exemple le chlorate de potasse d'après un procédé inspiré par celui de Schulz pour la destruction du ligneux.

Voici comment M. H. van Heurck conseille d'employer le chlorate de potasse.

Si l'on soupconne dans les matières la présence, de sels calcaires, ce qui arrive très fréquemment, il faut les traiter d'abord par l'acide nitrique pour dissoudre et enlever ces sels qui, par l'acide,

(1) L'eau distillée peut être remplacée par l'eau de pluie filtrée, beaucoup plus difficile, d'ailleurs, à avoir pure que l'eau distillée. 
sulfurique, se transformeraient en sulfate de chaux insoluble et resteraient mêlés aux Diatomées.

On place alors les matières débarrassées ainsi des sels calcaires dans une capsule de porcelaine et on les recouvre d'acide sulfurique. La capsule doit être assez grande parce que le mélange se boursoufle beaucoup. On chauffe avec précaution à l'aide d'une lampe à alcool, d'un bec Bunsen ou tout autre appareil, jusqu'à ébullition. On maintient l'ébullition pendant quelques minutes et l'on enlève la capsule du feu. On verse alors sur les matières, qui sont devenues noires et charbonneuses, une solution saturée de chlorate de potasse dans l'eau. On ajoute cette solution goutte à goutte; à chaque goutte il se produit une effervescence. On agite avec une baguette en verre et on ajoute une nouvelle goutte, jusqu'à ce qu'on ait employé une quantité de solution représentant à peu près la moitié du volume de l'acide sulfurique. A ce moment, la masse doit être tout à fait décolorée et les Diatomées débarrassées de toute matière organique. Dans le cas contraire, il faut recommencer.

Ajoutons que M. F. Kitton préfère il la solution saturée de chlorate de potasse le chlorate lui-même cristi lisi e! sec, parce que si l'on ajoute cette solution un ṕeu trop brusqu:cment, il se produit une effervescence très vive et le contenu de la capsule ou du tube peut être projeté au dehors, ce qui n'est pas sans danger en raison des propriétés extrèmement corrosives de l'acide sulfurique. II. F. Kitton opère dans un tube et, à la fin de l'opération, projette dans le mélange quelques petits cristaux de chlorate de potasse.

Quand la masse est blanchie, il fait des lavages succéssifs à l'eau pure jusqu'à ce que toute trace d'acide ait disparu. Alors, il ajoute 30 à 40 gouttes d'ammoniaque liquide concentrée et laisse agir pendant plusieurs heures, en fermant le tube arec un bouchon. Il y verse alors 13 grammes d'eau distillée et donne une brusque secousse au tube. Lorsque les Diatomées sont tombées au fond, il décante l'eau surnageante, ordinairement très trouble, et continue les lavages à l'eau distillée jusqu'à ce que toute trace d'ammoniaque ait disparu.

\section{Procédé par le permanganate de potasse et l'acide chlorhydrique.}

Ce procédé a été indiqué par M. Brun, de Genève. Voici comment il le décrit (1) :

"Si l'on a un magma frais de Diatomées encore humides, on y

(1) Journal de Micrographie, T. VI, 1882, p. 457. 
ajoute des cristaux de permanganate de potasse ec très peu d'eau (environ 1 partic de sel sur 10 d'eau); si l'on a des Diatomées desséehées, pures ou mélangées de terre ou de matières organiques, on les arrose d'une petite dose de solution du mème sel concentré, et eontenant mème quelques eristaux en exeès.

"La réaetion du permanganate doit durer environ 12 heures. Il est bon de remuer quelquefois le mélange mis au fond d'une fiole de 100 grammes, en moyenne, de eapaeité, et de plaeer eette fiole sur un fourneau ehaud ou au soleil. Il faut ensuite remplir à moitié d'eau la fiole, et ajouter un peu de magnésie calcinée (environ 50 centigrammes) que l'on laisse agir pendant 2 ou 3 heures en agitant quelquefois. Alors, on verse, par petites doses de 1 gramme au plus, et de 10 en 10 minutes, de l'aeide ehlorhydrique par. Lorsque tout le eontenu de la fiole s'est déeoloré, l'opération est terminée. Au bcsoin, pour faeiliter la réaction, on plonge la fiole dans l'eau ehaude ou bouillante. L'on proeède ensuitc aux lavages et décantation habitucls. Rappelons que la pureté absolue de l'eau distillée, pour ecs derniers lavages, reste toujours une condition essentielle de réussite.

" Dans ce proeédé, nous avons d'abord l'oxydation énergique de l'endoehrôme par le permanganate et la magnésie ; puis, par l'aeide, il y a dégagement d'oxygène gazerx (1) qui agit eomme eomburant, et ensuite de chlore qui agit comme déeolorant. C'est, sans doute, à ees réaetions multiples et sueessives, à l'extérieur et à l'intérieur même des valves, qu'il faut attribuer le nettoyage aussi parfait de leur silice.

«Par ce traitement les espèces délicates ne sont pas eorrodées, surtout si, avant l'action acide, on ajoute assez d'cau. La surfaec des valves a perdu tout son eoléoderme; elle apparait avec tout son éelat et les moindres détails, stries ou ponctuations, se distinguent nettement.

" J'ai essayé, ajoute M. J. Brun, sueeessivement, ces dernières années, tous les différents proeédés physiques et chimiques qui ont été annoncés et je puis dire que je n'en ai trouvé aucun qui réussisse aussi eomplètẹment et aussi régulièrement. »

\section{Procédé de M. Kitton pour les dépôts marins (2).}

Le nettoyage des Diatomées eontenues dans les dépôts marins

(1) Et même d'oxygène à l'état naissant, oủ ses propriétés comburantes sont beaucoup plus énergiques qu'à l'état simplement gazeux. Il en est demême pour le chlore. - (J.-P).

(2) H. van Heurck, Synopsis des 'Diatomées de Belgique, p. 28. 
est plus difficile, car elles sont souvent réunies par une sorte de ciment siliceux très difficile à enlever sans détériorer les Diatomées. M. F. Kitton opère de la manière suivante :

Il traite d'abord la matière par l'acide nitrique, porte à l'ébullition, pour enlever les sels calcaires, lave à l'eau distillée, et fait agir l'acide sulfurique, puis le chlorate de potasse pour brûler la masse eharbonneuse. Après un lavage complet, il fait bouillir la masse dans une petite quantité d'eau contenant un peu de carbonate de soude, lave et secoue fortement le tube pour désagréger les matières. Si la désagrégation ne se fait pas, il fait bouillir de nouveau dans une solution de potasse caustique, et, quand la masse est désagrégée, il verse le tout dans de l'eau contenant de l'acide chlorhydrique. Puis, derniers lavages.

\section{Procédé de M. F. Kitton pour les guanos, les sondages marins et les Diatomées fossiles.}

On commence par le traitement ordinaire à l'acide nitrique et quand toute trace d'acide a été enlevée par les lavages successifs, on fait bouillir toute la masse pendant trois à quatre minutes dans une trentaine de gramimes d'eau contenant un morceau de savon gros comme un pois. Qủand les Diatomées sont tombées au fond du vase, on décante l'eau savonneuse et on fait bouillir dans de l'eau pure. Le résidu ne doit contenir que les Diatomées et du sable.

Nous indiquons plus loin comment M. Kitton opère le triage des Diatomées et du sable.

Procédé de M. J. Brun pour les vases marines et les guanos.

« Un grand nombre de travaux récents de microscopie, dit M. J. Brun, ont eu pour but l'étude des récoltes pélagiques, celle des vases laeustres et marines et celle des dépôts fossiles. On y trouve des Polycystines, Radiolaires, Globigérines, Foraminifères, Spongiaires, Diatomées, et bien d'autres organismes infiniment petits et très variés. Dans les vases marines que ramène la sonde, on ne trouve guère que des espèces mortes et mème momifiées. C'est à la surface des mers et des lacs qu'est la vie! là où l'air et la lumière abondent. Et, si dans les vases profondes on trouve ẹa et là des exemplaires en bon état, c'est qu'ils viennent de la surfáce et qu'il n'y a pas longtemps que leur vie a eessé.

"Le naturaliste éprouve souvent de grandes difficultés à séparer ces organismes de la masse pulvérulente ou cristalline (siliceuse, argileuse ou calcaire), qui compose la plus grande partie des 
sondages. Ces substances, souvent mêlées depuis des siècles aux détritus organiques, forment ordinairement une masse pâteuse ou plastique, quelquefois même goudronneuse, très gènante et diffieile à séparer. On y trouve aussi souvent une forte dose de cendres voleaniques. Quelquefois mème le sondage n'est composé que de matières minérales sans traces d'organismes.

"Pour l'étude des organismes à carapaces silieeuses, Polycystines, quelques Radiolaires et surtout des Diatomées, il est indispensable de détruire entièrement cette eneombrante matière organique. Dans les guanos, les détritus chitineux abondent. Ceux-ei sont extraordinairement résistants à la putréfaetion et aux dissolvants, et mème aux acides chlorhydrique, azotique, et au chlore. - Tous ces détritus organiques, par leurs pesanteurs spécifiques variées et surtout par la grande adhérenee qu'ils ont contractée avec les particules minérales, empêehent également de pouvoir agir par lévigation.

«Le procédé suivant permet la destruction complète de toute cette matière organique, et comme il ne donne pas de vapeurs acides, il a l'avantage de ne nécessiter ni un laboratoire spéeial, ni une cheminée à fort tirage. Il donne aussi des résultats meilleurs que les traitements au chlorate de potasse, au permanganate ou à l'aeide azotique, ordinairement employés, et qui tous dégagent des rapeurs acides et corrosives.

“ La masse desséchée (pulvérulente ou compacte), est traitẻe dans une fiole avec de l'acide chlorhydrique aqueux, afin d'éliminer le calcaire. La fiole doit ètre d'une dimension suffisante pour contenir toute l'écume visqueuse que les calcaires donnent lorsqu'ils sont-ainsi intimement liés à une masse organique en déeomposition. La dissolution de ces sels terminće, le liquide et la vase sont jetés sur un filtre où le dépôt insoluble est lavé, puis desséehé, sur le filtre mème.

" Ce dépôt sec est alors mis en fiole et arrosé de deux fois son volume d'acide sulfurique concentré, qu'on laisse agir plusieurs heures en agitant quelquefois. La masse noircit. Pour les guanos il faut 5 ou 6 fois leur volume d'acide sulfurique. Cet acide est le seul qui dissolve bien des débris chitineux, et on peut déjà en éliminer la plus grande partie en décantant les $3 / 4$ du liquide sulfurique après un repos suffisant. Sur ce brouet épais et noiràtre on ajoute alors du bichrômate de potasse en poudre grossière. On l'ajoute par petites doses successives et en agitant chaque fois. La masse s'échauffe et souvent il y a dégagement d'oxygène. On s'arrête quand du noir elle a viré au rouge ou qu'il y a formation de cristaux rouges d'acide chrômique. Dans ce traitement, les matières organiques sont carbonisées par l'acide sulfurique, et 
c'est l'acide chrômique à l'état naissant qui en achève la comburation. Le lavage préalable à l'eau chlorhydrique a pour but d'éviter la formation de sulfate de chaux.

" Le liquide précédent est additionné peu à peu d'eau. La masse s'échauffe à nouveau. L'eau est ensuite ajoutée en abondance. Le dépôt restant est devenu plus ou moins blanc. On le lave soigneusement par décantation. Les dernières décantations se font à l'eau distillée. Il est alors prêt à ètre utilisé. Pour cela, on le délàye avec de l'eau distillée, et on verse ce mélange sur de grands couvreobjets (covers) sur lesquels on le dessèche. C'est sur ces covers que se trient les espèces (1).

\section{§ 2. - TRIAGE DES DIATOMEES}

Dans une récolte composée d'un certain nombre d'espèces, il est utile de pouvoir trier les espèces, afin d'en faire des préparations séparées. Si ces espèces sont de taille différente, on pourra profiter de ces différences de taille pour opérer une division entre les petites et les grandes espèces. - En mème temps, on pourra ainsi séparrer les Diatomécs des grains de sable plus ou moins fins et lourds qui ont, comme elles, résisté à l'action des réactifs, car ils sont comme elles composés de.silice.

Les grains de sable formés d'une substance plus condensée, sont plus lourds que les Diatomées de même volume. Et, parmi les Diatomées elles-mêmes, les plus grosses sont génćralement les plus lourdes.

Tous les procédés de séparation des Diatomées et du sable et des Diatomées entr'elles sont fondés sur ce principe, qu'on opère d'ailleurs sur des matériaux déjà passés aux réactifs, comme nous l'avons indiqué, ou sur des matériaux crus, c'est-à-dire bruts ou seulement débarrassés mécaniquement des corps étrangcrs les plus volumineux.

On comprend que, pour ce triage, on opèrera par lévigation ou décantation, en agitant les matic̀res dans l'cau et en séparant successivement les différents dépôts qui șe forment, les corps les plus lourds se déposant les premiers. Quant à l'argile, s'il y en a, elle restera au contraire cn suspension dans l'eau de lavage, pendant très longtemps au moins, si cette eau est distillée, et on pourra l'enlever par décantation.

Enfin, il cxiste des procédés et même des instruments ou doigts mécaniques qui permettent de choisir pièce à pièce dans un

(1)- J. Brun. - Journal de Micrographie, T. XI, 1887, No 5. 
groupe de Diatomées les spécimens que l'on veut monter, et même de les ranger dans la préparation, suivant un ordre voulu et dans une position déterminée. On fait ainsi ce qu'on appelle des préparations méthodiques.

Occupons-nous d'abord des procédés de triage du sable et des Diatomées, nous verrons plus loin à l'aide de quels artifices on arrive à manier, pour ainsi dire, chacun de ces corpuscules presque invisibles et à les disposer comme on le veut dans les préparations.

\section{Procédé de M. H. L. Smith pour le triage des Diatomées.}

Le professeur H. L. Smith a publié les instructions suivantes pour le triage des Diatomées. Il opère sur des matériaux crus et qui n'ont pas été traités par les acides, mais son procédé est évidemment applicable aussi aux matériaux déjà traités par les acides. II. H. L. Smith se dispense de ce traitement parce qu'il détruit la matière organique des Diatomées en brùlant celles-ci sur la lame de verre au moment du montage, comme nous l'indiquerons plus loin.

« Les récoltes, dit M. H. L. Smith, ne doivent pas être séchées, mais conservées humides, dans des fioles, avec un peu de créosote pour empêcher les moisissures. - Je préfëre de beaucoup examiner les frustules entiers avec les deux valves adhérentes, ou encore attachés les uns aux autres, si l'espèce est filamenteuse. Je possède beaucoup de fioles de matériaux pour le montage, qui sont presque aussi propres que s'ils avaient été traités par les acides...

"Supposons donc qu'on ait devant soi une fiole contenant une quantité d'eau considérable relativement au sédiment, lequel renferme plus ou moins de Diatomées. Nous procédons comme il suit, et si la fiole est restée tout à fait au repos pendant plusieurs jours, cela n'en vaudra que mieux.

" On imprime à la fiole un rapide mouvement de rotation: les matériaux les plus légers s'élèvent dans l'axe du liquide et se répandent bientòt dans l'eau.

" On laisse reposer pendant deux ou trois secondes, jusqu'à ce qu'on ait vu les plus grosses particules se déposer; aussitòt, on verse le liquide avec les particules flottantes qu'il contient dans une autre fiole. C'est de ce liquide que nous allons séparer les Diatomées et le sable de l'argile et des matières organiques.

"Les matériaux décantés dans la seconde fiole sont laissés au repos jusqu'à ce que l'eau paraisse simplement laiteuse ou nuageuse. Le temps varie avec la petitesse des Diatomées et l'on ne peut guère l'apprécier que par expérience. Supposons une minute: alors, tout 
ce qui reste flottant doit ètre décanté et jeté, à moins qu'il ne s'y trouve de très petites formes que l'on peut vouloir séparer.

" La fiole est de nouveau remplie arec de l'eau pure ou distillée (les eaux dures ou calcaires doivent ètre strictement écartées) - et l'on secoue de nouveau.

« Aussitôt que le dépòt le plus lourd touche le fond, le reste est versé dans une troisième fiole, en laissant environ le quart du liquide dans la seconde.

"Cette troisième fiole ne contiendra plus guère maintenant que du sable et des Diatomées avec quelque légère matière organique et de l'argile pure. Ces deux dernières substances peurent être enlevées par décantation: pour cela, on remplit d'eau la fiole $n^{\circ} 3$ et, après l'avoir bien secouée, on la laisse reposer de deux à cinq minutes; on décante et on jette l'eau légèrement laiteuse, et on répéte l'opération en laissant reposer un peu plus longtemps. On peut recommencer une troisième fois et rejeter les particules en suspension après un intervalle de 8 à 10 minutes.

« Souvent, après que le premier dépòt s'est formé dans la fiole $n^{\circ} 2$, les Diatomées s'élèveront plus pures dans la masse en donnant à la bouteille un mouvement de rotation qu'en la secouant. »

M. H. L. Smith ajoute à cet exposé les observations suivantes:

" Un peu de pratique et de soin permettra à tout le monde de séparer certaines Diatomées suivant leur taille. J'ai reçu une récolte de Pleurosigma Spenceri qu'on m'avait envoyée de Scioto River (Ohio), mais quoiqu'elle eut été bien traitée au chlorate, quand j'en montai une préparation, je ne trouvai pas plus d'un ou deux frustules dans le champ de microscope, le reste n'étant que des formes plus petites ou de fins fragments de silex. En étudiant et en expérimentant avec soin le temps pendant lequel les différentes tailles restaient en suspension, j'ai fait de cette récolte une préparation qui montre des centaines de frustules où l'on n'en voyait à peine auparavant, et l'on ne reconnaitrait pas qu'elle provient de la même récolte. "

M. H. L. Smith conseille ensuite, quand on a constaté la présence d'une assez grande quantité de Diatomées dans le dépôt, de remplir la fiole avec un mélange d'alcool et d'eau à parties égales, afin de les conserver pour le montage. Mais il faut avoir soin de n'employer qu'un alcool qui ne laisse pas de résidu par l'évaporation et que de l'eau distillée, sans quoi les préparations qu'on ferait avec ces Diatomées seraient mêlées de corps étrangers.

Quant au montage, l'éminent diatomiste américain le pratique, comme nous le verrons plus loin, en brûlant les Diatomées sur le cover, d'après la méthode de Brébisson, ce' qui détruit en mème temps leur matière organique. 


\section{Procédé de triage de M. F. Kitton.}

Nous avons indiqué précédemment (p. 112) les procédés qu'emploie M. F. Kitton pour le lavage des récoltes, des sondages marins, des Diatomćes fossiles et des guanos. Tous ces procédés d'aillcurs, quels qu'ils soient, arrivent toujours au même résultat : avoir dans de l'eau un dépòt qui ne contient plus, si les opérations ont été bien faites, que des Diatomées débarrassées de matière organique, et du sable.

En secouant le vase qui contient ce résidu et en décantant, au bout de quelques secondes, l'eau surnageante, on séparera déjà un premier dépôt qui contiendra les grains de sable les plus volumineux et les Diatomées les plus lourdes.

L'eau surnageante, abandonnée au repos, laissera déposer les Diatomées légères et les grains de sable les plus fins dont la sćparation est plus difficile. Voici comment M. F. Kitton opère pour faire ce triage :

" Je prends deux lames de verre A et $\mathbf{B}$. Sur $\mathbf{A}$ je place une goutte d'eau distillée et sur $\mathbf{B}$ une petite quantité du liquide à traiter. J'imprime à $\mathbf{B}$ un léger mouvement rotatoire horizontal, ce qui accumule le sable au centre du liquide et j'incline la lame vers un de ses angles; les Diatomées̀ s'écoulent et sont recueillies dans l'eau distillée de la.lame A. - Cette eau chargée de Diatomées est ensuitc répartie sur des couvre-objets.

" Par cette méthode, des récoltes qui paraissent tout à fait sans valeur donnent de très bonnes préparations, mais il est souvent nécessaire d'ajouter à l'eau de la lame A le produit de plusieurs gouttes de $\mathbf{B}(1)$. »

C'est encore là, comme on le voit, un procédé par décantation, mais dans lequel on opère sur de petites quantités, en profitant à la fois de la différence de densité du sable, et des Diatomées et de l'adhérence à la lame de verre des particules les plus lourdes.

A propos de cette adliérence au verre de certaincs particules, nous devons signaler une observation due au professeur Christopher Johnston. Pour les dépôts débarrassés ou à peu près de matières étrangères, si on les verse dans un vase en verre très propre, les Diatomées discoïdales, après quelques instants de repós, adhèrent au fond du vase et l'on peut décanter tout le contenu sans que celles-ci se détachent si elles sont entières. On peut alors les enlever avec un pinceau très doux, et obtenir ainsi des frustules parfaitement isolés.

(1) H. vax Heurck. - Synopsis des Diatomées de Belgique, p. 27. 


\section{VI}

\section{MONTAGE DES DIATOMEES}

\section{$\S$ 1. - INSTRUMENTS ET PRODUITS}

Le matériel pour le montage des Diatomées en préparation n'est ni compliqué ni dispendieux.

L'appareil le plus important est un petit support métallique, formé d'une tige de fer verticale sur laquelle glissent un ou deux bras terminés par un anneau. On peut fixer ces bras à une haulteur voulue, à l'aide d'une vis de pression, de manière à rapprocher ou éloigner l'anneau de la flamme d'une lampe à alcool ou à gaz qu'on place par-dessous. C'est, en somme, un instrument qu'on trouve dans tous les laboratoires, mais on doit le choisir de petite taille pour qu'il soit moins encombrant, et l'anneau doit ètre assez mince, pour ne pas absorber trop de chaleur, et de faible diamètre.

Sur l'un de ces anneaux on pose une plaque de fér assez épaisse pour qu'elle ne se gondole pas en se dilatant par la chaleur, $\check{\text { mil- }}$ limètres par exemple. On se munit aussi d'une petite lame de platine dont on replie les angles par dessous. Cette lame pourra ètre posée aussi sur un anneau du support.

Quelques pinces fines, parni lesquelles on peut en avoir une munie d'un anneau de serrage qui permet de chauffer directement la lame de platine en la tenant à la main avec la pince; tels sont, avec quelques aiguilles tous les instruments nécessaires.

r On se servira, d'autre part, de lames et de lamelles très propres, et l'on choisira les lamelles ou covers aussi. minces que possible afin de pouvoir étudier les préparations avec des objectifs à court foyer.

Les lamelles minces rondes sont plus commodes que les lamelles carrées, parce qu'on peut faire les cellules avec la tournette, ce qui est plus commode et plus expéditif.

On préparera, en effet, d'avance un certain nombre de lames de verre sur lesquelles on aura tracé avec du bitume de Judée, du vernis au blanc de zinc, à la gomme laque ou autre, un cercle de 
mème diamètre, ou un peu plus grand que le cover qu'on veut employer. - Tout le monde sait comment on trace ces cellules à l'aide de la tournette et la vue de l'instrument suffit à apprendre comment on s'en sert. Ajoutons seulement que les tournettes à centrage automatique, c'est à dire dans lesquelles la lame de verre se place de manière à ce que son centre coïncide toujours avec le centre du plateau tournant, sont préférables aux autres; en effet, la mème lame y reprend toujours exactement la même position, de sorte que les différentes couches que l'on veut donner à une cellule tombent toujours exactement les unes sur les autres (fig. i1).

On doit faire les cellules, avec le vernis qu'on aura choisi, vernis au bitume, au blanc de zinc ou autre, plusieurs jours ou mème plusieurs semaines d'avance et laisser sécher les lames de verre ainsi préparées à l'abri de la poussière. Ces cellules serviront pour monter les Diatomées à sec et dans un liquide conservateur.

\section{Vernis au bitume de Judée}

On le prépare en réduisant en poudre du bitume de Judée de bonne qualité et en versant par dessus de la benzine ou de l'essence de térébenthine. On agite avec une baguette de verre et on ajoute le liquide peu à peu jusqu'à ce que la solution ait la consistance d'un sirop un peu épais. On peut y mettre un peu de cire pour l'empècher de se fendiller par la dessication.

\section{Vernis au copal.}

On dissout de la résine copal dans de l'essence de lavande, en ajoutant de la résine et de l'essence jusqu'à consistance convenable.

\section{Baume du Canada.}

Le Baume du Canada est la résine de l'Abies canadensis. On l'emploie souvent tel qu'il se trouve dans le commerce. Il ne faut pas qu'il soit trop fluide ni trop filant.

On peut aussi s'en servir à l'état de dissolution dans l'essence de térébenthine ou dans le chloroforme.

Pour la préparation des Diatomées, le baume est préférable sans essence.

La solution de baume du Canada dans la benzine remplace avantageusement le vernis noir au bitume pour la confection des cellules.

\section{Résine Damar.}

On dissout la résine broyée dans l'essence de térébenthine ou le chloroforme, en donnant au mélange la consistance du baume du Canada. 


\section{Térébenthine de Venise.}

Elle s'employe comme le baume, c'est la résine de l'Abies pectinata.

\section{Veinis à la gomme laque.}

On dissout la gomme laque dans l'alcool absolu.

$$
\text { Mixtion des doreurs ou gold-size. }
$$

On trouve ce produit chez les marchands de couleurs. Il s'emploie comme le vernis au bitume. Il est long à sécher.

\section{Vernis au blanc de zinc.}

Le blanc de zinc est d'abord lavé dans la benzine. On filtre, et avant que la matière soit complètement sèche, on la broie dans une solution épaisse de résine Damar dans la benzine.

\section{Styrax, Liquidambar}

Le $\mathrm{D}^{\mathrm{r}} \mathrm{H}$. van Heurck a introduit, depuis quelques années, dans la technique le styrax (1) qui est la résine du Styrax orientalis, et le liquidambar, résine du Liquidambar styracifua, pour remplacer le baume du,Canada.

Le styrax du commerce est étendu. d'abord en couche mince sur des assiettes et exposé à l'air et à la lumière jusqu'à ce qu“il soit devenu assez dur et qu'il ait perdu l'enu qu'il contient; après quoi, on le dissout dans un mélange à parties égales d'alcool et de benzine ou d'éther sulfurique et d'alcool absolu. Et l'on filtre au papier. On peut aussi le dissoudre dans le chloroforme (1). Son indice de réfraction est 1,64 (Raie D) (2).

- Le liquidambar, difficile à trouver dans le commerce ordinaire, se prépare de la même manière, et en solution dans la benzine ou le chloroforme.

Nous indiquons plus loin les procédés de montage employés par M. H. van Heurck avec le styrax et le liquidambar.

\section{Baume de Tolu.}

C'est la résine du Balsamodendron toluifera.

Récemment introduit aussi dans la technique, M. J. Brun le

(1). H. van Heurck. Syn, des Diat. de Belyique, p. 29.

(2). J. BRux. Utilisation et valeur réelle des mediums. Journal de Micrographie. T. XI. 1887, p. 182. 
prépare en le faisant bouillir pendant longtemps dans une grande quantité d'eau et en le dissolvant dans la benzine rectifiée. On filtre et on laisse dessécher complètement la solution. Le résidu est enfin dissous dans l'alcool ou dans le chloroforme. Le liquide doit être limpide et concentré. L'indice de réfraction du baume de Tolu mou est 1,64 (raie D) et 1,72 pour le baume sec. Il est donc supérieur à celui du styrax.
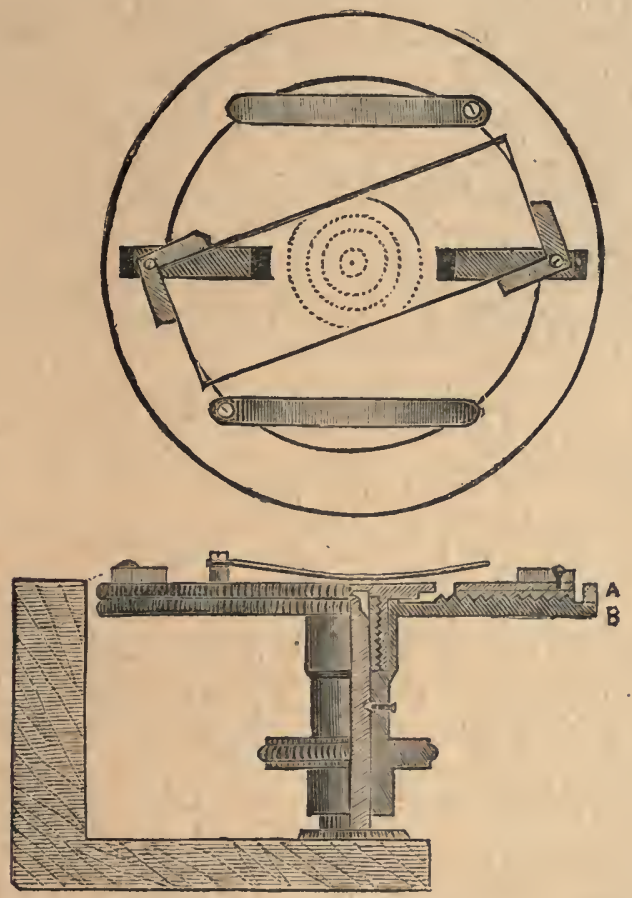

Fig. 73. - Tournette à centrage automatique, de W. Bulloch.

\section{Milieux à haut indice}

Dans ces dernières années, différents naturalistes ont cherché à produire des liquides, solidifiables ou non par le refroidissement, dont l'indice de réfraction soit supérieur à celui du baume. C'est ainsi qu'on a employé :

La naphtaline monobromée, (voir p. 136).

Les solutions de soufre et de phosphore dans le sulfure de carbone,

Le sulfure d'arsenic sublimé,

L'arsénite d'antimoine dissous dans le chlorure d'arsenic, 
L'acide arsénicux dissous jusqu'à saturation dans une gelće formée de bromure d'antimoine dans la glycérine; (indice 1,80 à $2,00)$,

L'acide arsénieux dissous dans une solution de sulfure d'arsenic (réálgar) dans le loromure d'arsenic; (indice $=2,30$ à 2,40 ).

Toutes ces substances, très désagréables à manier, parfois même dangereuses, n'ont pas grande valeur parce qu'elles ne sont pas stables et toutes celles qui renferment de l'arsenic, notamment, qui sont vitrifiables, (c'est sur cette propriété qu'est fondée leur emploi), éprouvent plus ou moins rapidement une dévitrification avec formation d'un magma de cristaux dans lequel la préparation est perdue. Ou bien, comme elles sont très avides d'eau, elles absorbent l'humidité de l'air et deviennent opaques.

Nous pensons done qu'il n'y a pas grand intérèt à se servir de ces diverses substances, à moins que ce ne soit pour quelque recherche extemporanće et qu'on ne tienne aucunement à conserver les préparations.

\section{Liquides conservateur's}

Eau camphrée. - L'eau camplirée est un des meilleur's liquides pour conserver les algues, en général, et les Diatomées, en particulier, dans les préparations.

On l'obtient en versant goutte à goutte de l'alcool camphré dans de l'eau distillée. A chaque goutte, il se produit un trouble; on agite avec une baguette de verre et le léger précipité de camphre se dissout. On opère ainsi jusqu'à ce que l'eau devienne définitivement opaline, la dernière goutte d'alcool camphré ne se dissolvant plus par l'agitation. On filtre alors sur le papier, et l'on conserve pour l'emploi le liquide filtré.

Chlorure de calcium. - On prépare une solution avec 3 grammes de chlorure de calcium pur dans 100 grammes d'eau distillée. On filtre et l'on ajoute une goutte d'une solution d'acide phénique pour empècher le développement des moisissures.

\section{$\$ 2 .-M O N T A G E$ A SEC}

Le montage des Diatomées à sec, c'est à dire sans l'intermédiairẻ d'aucun milieu, est le procédé le plus rapide.

Autrefois, on se bornait à déposer sur une lamelle coutre-objet bien propre une petite quantité des Diatomées destinées à ètre montées bien lavées, d'ailleur's, et conservées dans l'eau ou dans l'alcool. On prenait une gouttelette du dépót diatomifère, à la 
pointe d'un pinceau ou avec une pipette, et on ladéposait au milieu de la lamelle. On répartissait le mieus possible, avec une aiguille, la matière sur la lamelle, et on laissait sécher à l'abri de la poussière; ou bien, l'on liattait la dessication en approchant la lamelle de la flammie d'une lampe à esprit-de-vin. Le liquide étant complètement évaporé et la lamelle bien sèche, les Diatomées restaient adhérentes au verre. Il suffisait alors de déposer cette lamelle, la face chrargée de Diatomées tournée en dessous; sur une cellule tracée depuis longtemps à l'avance sur une lame de verre et bien sèche; of s'arrangeait de manièré que le cerclè de bitume ou autre vernis formant la cellule dépassầt un peu les bords de la lamelle. Alors, en exerçant sur celle-ci une dotice pression avec la pointe d'un canif ou d'une aiguille, on détermithait l'adhérence de la lamelle avee le ceréle de vernis: Ou bien, on déposait sur le couvre-objet un petit disque de jlomb agissant de même par son poids. L'adhérence étant obtenue; il ne restait plus qu'à termitier la préparätion en recouvrant les bords de la lamelle d'un cercle de vernis les dépassant un peu et sé confondant avec celui de la cellule. La préparation se trouvait ainsi fermée.

Actuellement, on préfère employer le procédé de Brébisson; par le brûlage.

Si les Diatomées sont conservées dans l'alcool aqueux, on dépose avec une pipette une goutte du liquide diatomifère sur un couvreobjet bien propre placé sur la plaque de fer ou la lame de platine dont nous avons parlé antérieurement. On chauffe alors avec la lampe à alcool. L'alcool prend feu, et en brûlant répartit les matières sur toute la surface de la lamelle. Quand il a brùlé, on baisse la flamme et on laisse l'eau qui reste s'évaporer lentement.

Si les Diatomées à mionter ne sont pàs conservécs dans l'alcool mais dans l'eau; on dépose ủne goulte du liquide diatomilère sur une lamelle placée sur la plaque de fer ou la lame de platine, et l'on chauffe doucement jusqu'à siccité; après avoir réparti le mieux possible les Diatomées sur la lamelle.

Dans l'un et l'autre caś; quảnd là lamelle est bien sèche, on chauffe au rouge la plaque fer et le couvré-objet qui y est placé, ou on le porte sur la lamelle de platine, et l'on chauffe au rouge:

Ce procédé permet de monter dés Diatomées qui n'ont été traitées ni par les acides, ni jar aucun des procédés employés pour détruire la matièrè organique, c'est à dire à l'état de ce qu'on appelle des matériauxi crus. On comprend que la chaleur rouge détruit la matière organique qui noircit d'abord, puis brùle; et les frustules silicéux se trouvent parfaitement nettuyés. Toutefois; cette méthode serait insuffisante pour détruire la gangite organique ct minéralè de certains dépòts; et des guanos; en particulier: On ne peut l'emploỵer 
que pour les Diatomées séparées des matériaux étrangers et ne contenant à peu près que la substance organique qui leur est propre.

Cette calcination effectuée, on éteint la lampe, on laisse refroidir lentement et on dépose la lamelle ainsi chargée, les Diatomées en dessous, sur une lame de verre portant une cellule au vernis, tracée d'avance, et l'on opère en tout comme nous l'avons indiqué plus haut.

Les Diatomées sont ainsi montées dans l'air pour milieu. En raicon de la grande différence entre l'indice de réfraction de l'air et l'indice de la silice qui compose les frustules, les détails de ceux-ci seront plus faciles à voir et, par exemple, leurs stries plus faciles à résoudre. Néanmoins, ces avantages sont compensés par une notable perte de lumière par réflexion sur la face inférieure du cover, et une moindre admission de rayons lumineux dans l'objectif.

De plus, ces préparations sont fragiles.

M. II. L. Smith emploie ce procédé du brủlage sur des Diatomées qui n'ont pas été traitées par les acides, et, le plus souvent, la matière organique est ainsi parfaitement brùlée.

Les Diatomées à monter étant dans l'alcool aqueux, il prend avec une pipette un peu du liquide qui les contient et en dépose une goutte au milieu d'un couvre-objet très propre. - Il faut autant que possible employer des lamelles très fines afin de pouvoir se scrvir pour l'étude des objectifs à court foyer.

\section{\$ 3. - MONTAGE DANS LES BAUMES}

Dn se servait autrefois de la térébenthine de Venise; on l'a remplacée, depuis, par le baume du Canada, mais les manipulations sont les mèmes. Par ce procédé, les Diatomées, au lieu d'ètre montées dans l'air, sous la lamelle, sont comprises dans une couche transparente de baume solidifié.

On commence, comme nous l'avons indiqué pour le montage à sec, par sécher ou brủler les Diatomées sur la lamelle en portant celle-ci au rouge sur la lame de platine.

D'autre part, on a préparé d'avance des lames porte-objets au centre desquelles on a déposé une goutte de baume, ou d'une solution épaisse de baume dans une essence, et particulièrement dans l'essence de térébenthine. On a chauffé ces lames de verre à une chaleur modérée sur la plaque de fer, pour faire évaporer les parties volatiles du baume et l'essence de manière qu'il puisse se solidifier par le refroidissemient.

En chauffant ainsi, il se forme dans la goutte de baume fondu 
une plus en moins grande quantité de bulles. La gouttc étant maintenue pendant quelques instants à l'état liquide, la plupart des bulles crèvent et se dissipent. Pour faire disparaitre celles qui persistent, on en approclıe une aiguille chauffée dans la flamme de la lampe, ou mème on les touche avec la pointe de cette aiguille. Elles crèventalors et disparaissent. S'il cn reste une ou deux petites dont on ne peut se débarrasser, il ne faut pas trop s'en préoccuper ; le plus souvent, elles seront cliassées au delà des bords du cover quand on appliquera celui-ci, ou bien, formées de vapeurs d'essence, elles se résorberont avec le temps en se dissolvant dans le baume.

Quant au cover, les Diatomées qui le couvrent ayant été chauffées au rouge avant qu'il soit complètement refroidi, on y dépose une petite goutte d'essence de térébenthine ou de girofles, ct l'on chauffe pour l'évaporer. Quand clle est presqu'entièrement disparue et que lc cover n'est plus que légèrement humecté par l'essence, on le prend, chaud encore, avec une pince et on lc dépose, les Diatomées en dessous, sur la goutte de baume séchéc au milieu du porte-objet.

On chauffe doucement, le baume se ramollit et adhère, sans former de bulles, au cover mouillé de térébenthine. On éteint la lampe et l'on exerce avec la pointe d'une aiguille une légère pression sur le cover ou bien on le charge d'un petit poids en plomb pour diminucr l'épaisseur du baume sous la lamelle. Le baume en excès sort par la pression, autour du bord de la lamelle. On laisse le tout au repos jusqu'à ce que la solidification soit complète.

Quelques jours après, on gratte avec un canif le baume extravasé qui est sec et solide, et on nettoie la préparation avec un linge fin imbibé d'alcool concentré.

Le petit artifice, qui consiste à mouiller lc cover diatomifère avec un pcu d'essencc de térébenthine avant de l'appliquer sur le baume, a une grande importance. La légère couche d'esscnce qui l'luumecte permet une adhérence complète avec le baume sans interposition de bulles d'air. De plus, l'essence pénètre toutes les anfractuosités, dessins, stries des frustules, et en chasse l'air qui pourrait y ètre emprisonné, de sorte que le baume à son tour pcut s'insinuer dans toutes ces anfractuosités en se dissolvant dans la petite quantité d'essence qui les remplit. Enfin, cette mince couclie d'essence détermine une adhérence des Diatomées au cover suffisante pour que celles-ci ne tombent pas pendant le retournement et les manipulations.

Les préparations ainsi faites sont très solides et se conservent indéfiniment. Aussi, est-ce le procédé le plus employé, mais, comme le baume pénètre tous les détails de structure des frustules et que son indice de réfraction est peu différent de celui de la silice 
transparente qui compose ces frustules, ceux-ci deviennent beaucoup plus difficilement visibles que dans les préparations à sec oụ dans l'air. Ils forment av́ec le baume une masse à peu près homogène au point de vue aptique, et, pour étudier leurs détails, il est nécessaire d'employer les instruments les plus perfectionnés.

\section{Preparations au styrax et au liquidambar}

Le styrax et le liquidambarar ont un indice de réfraction supérieur à celui du baume du Canada. Les Diatomées y sont donc plus facilement visibles que dans le baume ; elles s'y montrent en vertu de la différence des indices de réfraction et par un phénomène analogue à celui qui rend visible une bulle d'air dans l'eau.

Nous avons indirgué plus hạut comment le $D^{r} H$. Van lleurck prépare ces deux substances. Voici comment il opère pour la préparation des Diatomées:

« Nous commençons par placer les couvre-objets sur une grande plaque de yerre et sur chacun d'eux nous plaçons, à l'aide d'une pipette, une large goutte d'eau distillée sur laquelle nous laissons tomber doucement une goutte du liquide diatomifère (1). Les Diatomées s'éparpillent dans la goutte d'eau distillée qui, au besoin, est remuée délicatement. - Les couvre-objets sont recouverts d'une cloche de verre et abandonnés à l'évaporation spontanée.

"Lorsque celle-ci est parfaite, les couvre-oljets pris un à un sont chauffés au rouge sur la lame de platine et remis sur la grande plaque de verre, où, aprẹs avoir reçu une goutte très fluide de styrax, ils sont de nouveau abandonnés, sous la cloche, à l'évaporation.

" Peu d'instants après, la couche hlanchit, mais on n'a plus à s’inquiéter de ce phénomène (qui n’arrive pas arec la solution dans le chloroforme), et, au bout de 24 heures, la benzine est complètement évaporée. Le couvre-objet est alors placé, retourné, sur le porte-objeț et ehauffé légèrement, de préférence sụ un pạin-marie, Une légère pression à l'aide d'une presselle chạsse les bulles d'air, s'il y en a, ainsi que le styrax superflu que l’on enlève après refroidissememt (2),

On peut opérer de même avec le liquidambar que l'on emploie dissous dans la benzine ou le chloroforme;

(1). Si les Diatomées sont conservées dans l'alcool, il faut décanter l'aleool et la remplacer par de l'eau distillée.

(2) H. Van Hzurck. -- Syn; des. Dint. de Belg. p. 29. 


\section{Préparations dans le baume de Tolu.}

Nous avons indiqué le mode de préparation du baume de Tolu (roir page 120) d'après J. Brun; cet auteur l'emploie de la manière suivante :

Quand les lamelles sont chargées des Diatomées à monter, après dessication ou brùlage, on dépose sur celles-ci, avec une baguette de verre, un peu d'une soluton très liquide de tolu dans la benzine. Cette solution est destinée à agir ici comme la térébenthine déposée sur la lamelle pour le montage dans le baume du Canada. Les Diatomées sont ainsi pénétrées par le liquide balsamique. On laisse sécher sous une cloche, dans un endroit chaud, ou mieux dans une étuve à $60^{\circ}$ ou $70^{\circ}$. Dans ces conditions, l'éraporation de la benzíne est complète en une ou deux heures. On peut le plus souvent alors, sans autre manipulation, retourner le cover sur un porte-objet propre et chauffer doucement par dessous. Le tolu de la lamelle fond, et en exerçant une pression celle-ci adhère au porte-objet.

Dans d'autres cas, on a placé un peu de solution alcoolique ou chloroformique de tolu sur le porte-objet avant d'y appliquer la lamelle. On opère ainsi comme avec le baume du Canada.

\section{$\S$ 4. - MONTAGE DANS LES LIQUIDES}

On monte les Diatomées dans les liquides conservateurs lorsqu'on veut les préparer avec leurs caractères botaniques, leur thalle, thalame, tube ou pédicelle, et particulièrement quand on veut les conserver in situ, par exemple sur les plantes qui les supportent.

On se sert alors de porte-objets portant une cellule tracée d'avance, cellule dont l'épaisseur doit être un peu plus grande que pour les préparations à sec. C'est-à-dire que l'on fait à la tournette un premier cercle de vernis sur la lame de verre, et quand il est sec, on en applique un second par-dessus; au besoin, un troisième. On peut aussi employer des cellules faites avec des anneaux découpés dans du papier d'étain, dans une feuille de cire et surtout dans des lames de verre très minces. Ces anneaux sont collés d'avance sur le porte-objet avec différents ciments ou tout simplement avec du baume du Canada.

On dépose dans la cellule une goutte du liquide conservateur dont on veut se servir et on met dans cette goutte, avec la pointe d'une aiguille, un pinceau ou une pipette, les Diatomées qu'on -veut préparer. S'il est nécessaire, on place la préparation sous une loupe 
montée, afin de pouvoir donner aux spécimens une disposition convenable. Après quoi, on prend un couvre-objet bien propre, on humecte sa face inférieure avec l'haleine et on le pose, non pas à plat, mais par un mouvement de rabattement, sur la cellule pleine de liquide. Les bulles, s'il s'en forme, sont chassées en dehors de la cellule, et il n'y a plus qu'à obtenir l'adhérence du cover avec les bords de la cellule. Si cette cellule a été faite avec un cercle de vernis, l'adhérence se produit en maintenant une certaine pression sur le cover jusqu'à ce que.le liquide qui mouille le vernis soit évaporé. Si la cellule est faite avec une rondelle métallique ou une lamelle de verre, on a dủ prendre d'avance la précaution d'enduire le dessus de cette cellule de vernis ou de baume de manière à ce que le cover puisse y adhérer.

On.a soin, d'ailleurs, d'essuyer, avec un peu de papier brouillard ou un linge fin, le liquide en excès qui déborde de la cellule après le rabattement du cover, et quand l'arhérence est obtenue, on la maintient par un cercle de vernis appliqué sur les bords de manière à fermer complètement la cellule. Quand ce cercle est sec, on peut en donner un autre, pour plus de sureté. Et la préparation est achevée.

\section{§ 5. - PRÉPARATIONS SYSTÉMATIQUES}

Tout le monde connait les préparations dites systématiques, dans lesquelles les Diatomées - ou autres objets microscopiques - sont, à l'aide de procédés particuliers, rangés dans un ordre déterminé. Les plus célèbres sont les fameux Typenplatte de J. Möller, qui présentent, les uns 20 Diatomées types, ou tests alignés suivant un ordre toujours le mème, les autres 100 et même 400 Diatomées classées méthodiquement. Les procédés employés par M. J. Möller sont restés secrets et aucun préparateur, que nous sachions, n'a encore obtenu des résultats aussi parfaits que l'opérateur allemand; toutefois, on trouve maintenant dans la commerce des plaques-types contenant jusqu'à 200 Diatomées classées (1) qui peuvent remplacer les Typenplatte de Möller. Beaucoup de diatomistes s'occupent aujourd'hui de ce genre de travaux, non seulement pour ranger dans un ordre déterminé plusieurs espèces dans une même préparation, mais surtout pour séparer les espèces les unẹs des autres et les disposer chacune à part dans des préparations distinctes.

(1) On trouve ces préparations systématiques au laboratoire du Journal de Micrographie, à Paris 
Pour résoudre ce problème, il faut pouvoir choisir dans un mélange de Diatomées tel et tel frustule, l'enlever, le porter sur une lamelle destinée à le recevoir et l'y déposer à une place donnée. En outre, il faut que le frustule, une fois déposé à l'endroit choisi, y reste et ne puisse pas se déplacer.

Plusieurs instruments ont été inventés qui permettent de manier ainsi les objets microscopiques, pièce à pièce, et de les ranger comme on le désire. Le plus connu de tous est le doigt mécanique (mechanical finger.) de M. J. Zentmayer, de Philadelphie, que nous avons présenté à l'Exposition Universelle de 1878 à Paris. Malheureusement, cet appareil, qui parait le plus satisfaisant de tous, est assez compliqué et d'un prix élevé. Mais, pour les besoins ordinaires de leurs études, les diatomistes emploient des appareils plus simples qui leur permettent d'arriver à peu près aux mêmes résultats : par exemple, de trier les frustules dans un mélange, d'en faire des préparations séparées.

Il y a déjà longtemps, Bourgogne le père, qui avait d'ailleurs une grande adresse de main et une longue habitude de ces travaux délicats, faisait des triages de Diatomées en se servant d'un pinceau composé d'un seul poil, - si cela peut encore s'appeler un pinceau, - et l'œil armé d'une loupe d'horloger à fort grossissement, il séparait avec habileté les plus grosses espèces contenues dans un mélange.

C'est, en somme, son procédé qu'on a perfectionné.

On emploie pour manier les frustules un poil emmanché : on s'est servi d'un poil de blaireau, coupé court, puis d'un morceau de poil de barbe, puis d'un cil; aujourd'hui, on emploie surtout un cil de chien ou de porc, fixé solidement au bout d'un manche ou saisi à demeure dans les mors d'une pince fine; puis, on opère sous un microscope armé d'un objectif d'un pouce de foyer et d'un oculaire très fort, ce qui permet d'obtenir un grossissement suffisant pour bien distinguer les objets, tout en conservant une distance focale assez grande pour manœuvrer sous la lentille.

Il faut pouvoir fixer à la platine, à l'aide d'une vis de pression une sorte de plateau ou de rallonge pour soutenir la main qui opère à la hauteur de la platine, ou bien l'on place près du microscope un bloc de bois d'une hauteur convenable pour soutenir la main et éviter le tremblement résultant de la fatigue.

Les Diatomées à trier sont placées sur une lamelle, sous l'objectif, et, à còté, on place une autre lamelle sur laquelle les frustules choisis doivent être déposés.

Il y a donc là une première difficulté, qui est de faire tenir les deux lamelles dans un mème champ de microscope, ce qui force, comme nous l'avons 'dit, à n'employer qu'un objectif faible, le 
grossissement étant obtenu par l'oculaire et l'allongement du tube. De plus, il est difficile de se servir d'un prisme redresseur, qui serait cependant utile, car le mieroscope renverse les objets et les mouvements. Nais le prisme redresseur diminue beaucoup le champ. D'ailleurs, on prend très vite l'habitude d'opérer dans l'image renversée, et toutes les personnes qui ont disséqué sous le microscope composé savent travailler dans ces conditions.

Il faut encore que la lamelle sur laquelle on porte le frustule pris au bout du poil soit recouverte d'un léger enduit agglutinatif qui y fasse adhérer le frustule et qui ne lui permette plus de se déplaeer.

M. Huyttens qui, l'un des premiers, s'est occupé du maniement des objets microscopiques, passait sur la lamelle le bout du doigt portant une gouttelette de glycérine. Le léger enduit de glycérine, le transport achevé, était chassé par la chaleur.

M. Bourgogne père se servait de ce qu'il appelait son encollage faible auquel M. H. Yan Heurck a substitué une couche très mince d'une solution de colle forte pure, solution un peu moins épaisse que celle dont se servent les menyisiers. La solution est appliquée à chaud sur le cover chauffé aussi; on la laisse sécher à l'abri de l'air. Au moment de l'emploi, on ramollit l'enduit en chauffant légèrement la lamelle, la face enduite en dessus. Et l'on peut répéter l'opération aussi souvent qu'on le veut, si l'on désire ajouter de nouveaux frustules sur la préparation.

Quand le dépôt des Diatomées est achevé, on chauffe un peu, l'enduit se ramollit, les frustules s'y collent à demeure.

On achève alors la préparation en déposant sur le cover une goutte de vernis. au copal et à l'essence de lavande, ou de styrax, ou de baume du Canada, et on l'applique sur un porte-objet comme nous l'avons indiqué pour les préparations dans les baumes.

La couche de colle étant extrimement mince, elle ne gêne aucunement pour l'étude des Diatomées au microscope, bien que son indice de réfraction $(1,32)$ soit un peu inférieur à celui du verre.

i. M. J. Brun recommande pour encollage la glycérine adragantée préparée ainsi :

On traite 1 gramme de gomme adragante blanche, pulvérisée, par 50 grammes d'eau distillée bouillante qui dissout seulement la moitié de la gomme. On filtre et on ajoute au liquide filtré un volume égal de glycérine très pure.

I. J. Brun dépose sur la lamelle á monter une très petite goutte de glycérine adragantée et c'est dans cette goutte qu'il porte, avec la pointe du cil emmanché, les frustules choisis. Le peu de colle qui reste à chaque fois au bout du cil facilite l'adhérence des frustules que l'on veut saisir.

Quand le triage est opéré, il ajoute un peu d'eau distillée à la 
gouttelette de gomme avec un petit pineeau bien lavé, il enlève les poussières avec un cil robuste et dispose les Diatomées sur le cover. Il fait alors volatiliserla glycérine au bain-marie ou dans une étuve à $100^{\circ}$. Les frustules adhèrent au verre, gràce à la très petite couche de gomme qui y reste. L'indice de réfraction de la gomme adragante est sensiblement égal à celui du verre $(1,52)$.

\section{Appareils et procédés de M. Rataboul.}

M. Rataboul a inventé divers petits instruments qui facilitent beaucoup ces délicates manipulations et permettent de ranger les Diatomées aussi régulièrement qu'avec le doigt mécanique de Zentmayer.

Le premier de ces instruments, qui sert pour le triage des grosses espèces, est composé d'une planchette épaisse A (Pl. III, Fig. 1) servant à donner à l'appareif la sțabilité voulue, Un bloc de bois B à pans eoupés s'y trouve fixé et sert à soutenir les mains, comme les supports à plans inclinés des microseopes simples actuels, Dans un angle de la planchette est une tige verticale en laiton D, portant une branche horizontale doublement articulée, à l'extrémité de laquelle est monté un doublet ou une loupe Coddington $F$, qui peut ainsi s'élever ou s'abaisser et prendre toutes les directions que l'on veut (1).

Sur le sommet plat du bloc de bois est fixée la platine sur laquelle on opère. C'est un secteur en euivre G épais de 3 millimètres, noircị sur sa face supérieure. Il est fixé sur le bloc par une vis à large tête plate $\mathrm{V}$, mais pas assez serré pour qu'il ne puisse tourner dans son plan autour de cette vis comme eentre. Il est, en outre, percé de deux trous ronds $(b, c$, Fig. 2$)$, inégaux, mais dont les centres sont situés à la mème distance du centre de la vis $\mathrm{V}$, de sorte que les rayons $V b$ et $V c$ sont égaux. Il est bon que cette platine tournante soit assez grande pour que si, dans le transport des Diatomées, on laisse échapper un frustule, il tombe sur la platine et ne soit pas perdu.

On comprend facilement le mode d'emploi de ee petit appareil. Le cover sur lequel on a déposé les Diatomées à trier est fixé sur le plus grand trou $c$ de la platine, et celui sur lequel on veut placer

(1) Cette double articulation peut être réalisée d'une manière bien simple. $\mathrm{La}$ pièce E, qui la représente, peut être un simple bouchon pereé, avec une lime queue de rat, de deux trous voisins et perpendiculaires. Dans l'un on enfonce à frottement dur la tige verticale $\mathrm{D}$ et dans l'autre le support horizontal du doublet. On peut ainsi élever ou abaisser la loupe en élevant ou abaissant le bouchon qui la porte sur la tige $\mathrm{D}$, comme on peut allonger ou raccourcir le levier horizontal en l'enfonçant plus ou moins dans le bouchon. Quand celui-ci est ușé par les frottements, on le rẹplace par un autre. 
les frustules choisis est collé sur le plus petit trou $b$. Sur ce petit cercle on trouvera aisément le centre, s'il s'agit, par exemple, d'y monter une seule Diatomée. On fixe les covers sur la platine avec un peu de paraffine que l'on fond à l'aide d'une petite tige métallique chauffée. On dispose alors la loupe au dessus du cover $c$. Avec le poil emmanché on choisit un frustule; puis, de la main gauche, on imprime un léger mouvemenl de rotation à la platine jusqu'à ce que le cover $b$ vienne se placer sous la loupe. On y dépose alors le frustule dans la position voulue; - et l'on recommence.

Il n'y a pas, ordinairement, de miroir. Les Diatomées se voient, en effet, plus distinctement sur un fond noir que sur un fond éclairé. Aussi, est-il avantageux de coller un peu de papier noir sous les ouvertures $b, c$, de la platine. Si, cependant, il est nécessaire d'éclairer par dessous, on peut disposer en $\mathrm{N}$ un petit miroir.

Cet appareil est très commode toutes les fois qu'on a à trier de grosses espèces, et que la loupe, qui ne renverse pas les images, donne un grossissement suffisant. Mais pour les très petites espèces, alors qu'il faut un grossissement de 100 à 1500 diamètres, on est obligé de remplacer la loupe par un microscope composé, en cherchant toujours à obtenir surtout le grossissement par l'oculaire pour avoir plus d'espace sous l'objectif.

L'appareil doit alors être modifié. La platine tournante est montée de la même manière sur une plaque de cuivre A (Fig. 3.) percée d'un trou à son centre. Cette plaque est fixée solidement avec des vis sur la platine du microscope de manière que son trou central, sur lequel on amènera successivement les ouvertures $b, c$, coïncide avec le trou de la platìne du microscope. On dispose, de chaque côté de l'instrument des blocs, devant servir d'appuie-mains, et l'on opère sous lẹ microscope composé comme on a fait sous le doublet, mais les images et les mouvements sont renversés.

Mais alors, il devient le plus souvent nécessaire, pour donner au poil des mouvements assez petits et assez sûrs, de le monter en doigt mécanique. On se sert alors d'un petit instrument dont le principe est dù à M. J. Chalon. ,

Une bande de cuivre $C_{i}$ (Fig. 74) formant bague ou ceinture, serrée par une vis $\mathbf{U}$, peut ètre fixée solidement à la partie inférieure du tube du microscope, au dessus de l'objectif. Elle porte sur un de ses côtés une tige $\mathrm{D}$ qui, par deux articulations à boule $\mathrm{M}$ et $\mathrm{S}$, soutient le poil emmanché Z. L'articulation $\mathbf{M}$ permet tous les mouvements à la tige T qui la traverse et dont on peut régler la longueur avec la vis de pression R. La boule de l'articulation S est traversée par la tige P qui porte le poil, et la longueur de cette tige peut de même être réglée en l'enfonçant plus ou moins dans la boule S. Le poil a donc, lui aussi, des mouvements dans tous les sens. On règle la hauteur de 
la tige $\mathbf{T}$ et la longueur de la tige $\mathrm{P}$ de manière à ce que le poil puisse manœuvrer sur la platine du microscope sur laquelle est adaptée la. plaque de cuivre portant le secteur et les covers fixés à la paraffine. On travaille alors sous l'objectif, avec le secteur tournant, comme on faisait précédemment sous le doublet, mais avec une grande sùreté de main et par des mouvements aussi précis et aussi petits qu'il est nécessaire (1).

Il est facile de comprendre que, Jorsqu'on emploie le doigt mécanique, certains mouvements, sont renversés et d'autres ne le sont pas, ou plutôt sont redressés par le microscope. Ainsi,

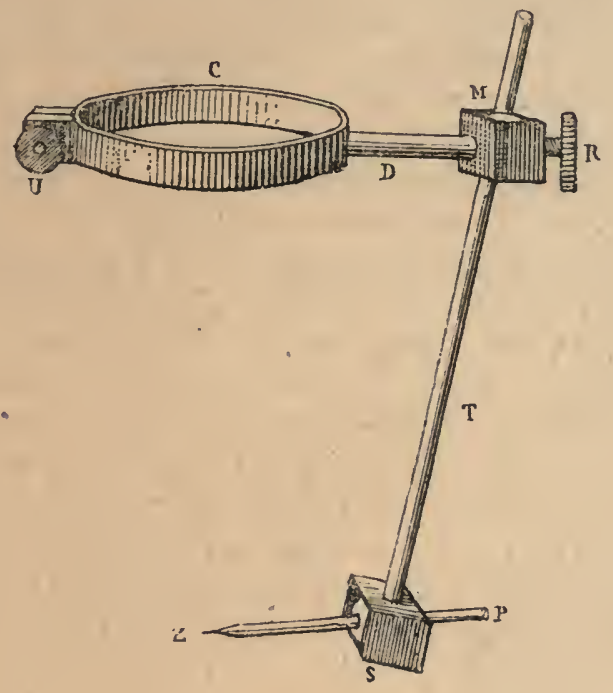

[Fig. 74. - Doigt mécanique.

dans la position de la figure, si l'on pousse la tige $\mathbf{P}$ vers la gauche, le poil qui la termine prendra réellement cette direction, mais le microscope renversera le mouvement et le poil, paraissant venir par la gauche du champ, s'avancera vers la droite. Mais si l'on pousse la tige $\mathbf{P}$ vers le devant du microscope, il se fera un mouvement de pivotement autour de l'articulation S comme centre et le poil viendra en arrière sous l'objectif ; mais le microscope renversera le mouvement et le poil paraitra dans le champ venant de l'arrière et marchant vers le devant du microscope, c'est-à-dire que l'image sera redressée. Dans ce dernier mouvement, il faut remarquer

(1) On trouve le doigt mécanique décrit ci-dessus au Laboratoire du Journal de Micrographie, a Paris. 
eneore que plus le bras de levier du poil (SZ) sera grand par rapport au bras de levier du manche (SP), plus le déplacement du poil sera grand par rapport au déplacement du manehe. Il faut donc une certaine habitude pour se servir aisément de cet instrument.

Avee le premier appareil ou avee le séeond, triant les Diatomées avec le poil emmanehé à main libre ou avec le doight mécánique; M. Rataboul opère de la manière suivante :

Les Diatomées à trier sont déposées avec une pipette sur un eover placé lui-mème sur la petite lame de platine M (PI. III, Fig. 1) et brúlées suivant le proeédé ordinaire. Puis, le cover refroidi est fixé avee un peu de paraffine sur le trou $c$ du secteur tournant. D'autre part, un autre cover très propre est fixé de méme sur le trou $b$. On dépose alors à son centre, avec un pineeau, une petite goutte d'une solution de gomme très pure. Cette solution est faite avee une grosse goutte de gomme arabique épaisse dans $1 \%$ e. cubes d'eau distillée. On laisse sécher l'enduit, et, au besoin, on hàte la dessieation en approehant du cover une tige mé‘allique chauffée au rouge. On fait alors le triage avec le poil, en amenant sous la loupe ou l'objectif le cover $c$, enlevant un frustule et le déposant sur le cover $b$ amené à son tour dans le ehamp par un petit mouvement du secteur tournant. On plaee le frustule où l'on veut, sur l'enduit gommeux see. Pour l'y fixer on prend un tube de verre $\mathrm{H}$ (Fig. 1) large par un bout, étroit par l'autre, et, la plus petite ouverture tournée vers la Diatomée, on projette l'haleine sur le eover dont l'enduit se ramollit assez pour que le frustule y reste collé.

On répète l'opération autant de fois qu'on le veut, et quand les Diatomées sont rangées et fixées, il n'y a plus qu'à monter la préparation, soit aux baumes, soit à sec.

Pour la monter dans le bàme du Canada, M. Rataboul détaehe le cover et le place pendant une heure, la faee chargée en dessous, dans un vérre de montre contenant de l'essence de térébenthine ou de lâvande.

Puis, il dépose surr une lamé porté-objei une goutte de baume. II la chauffe sự une lampe à aleoól, et quand des zébrures se montrent dans le bäume, il laissé refroidir. S'il y a des bulles, il les erève oü les enlève avee une aiguille froide. Le cover est alors retiré de l'essenee, égoütté ct déposé, les Diatomées en dessous, súr la gouitte de baume; à l'àide d'uñe chaleur ménagée, l'adhérence se produit, l'essence ch excès s'évapore. On comprime légèrement et on laisse refroidir. Il n'y a plus qu'à nettoyer la préparation quand elle est parfaitement sèehe, comme nous l'avons indiqué.

M. Rataboul reeommande le vernis àu copal, comme plús faeile à manier, quoique beaucoup plus long à séeher, parce qư'il est plus 
facile d'éviter les bulles d'air. Le vernis doit être évaporé jusqu'à: eonsistance sirupcusc, environ au tiers de son volume, et il suffit d'en déposer une goutte sur le porte-objet et de placer par dessus le eover imbibé de térébenthine. On laisse alors la préparation, pendant 2 ou 3 heures, sur une plaque de fonte légèrement ehauffée (à $70^{\circ}$ environ). Et l'on n'aura à eraindre aucune bullc. Il est prudent d'attendre quelque temps avant de nettoyer la préparation.

Pour les préparations à sec, M. Rataboul n'emploie pas la gomme pour enduire le cover, mais une gouttelette de baume dissous dans le ehloroforme, ou de vernis au copal, dissolutions d'ailleurs très étendues. Il opère, du reste, comme précédemment; mais au lieu d'utiliser l'humidité de l'halcine pour ramollir l'enduit et le rendre agglutinatif, il emploic une tige métallique chauffée qu'il approche du eover.

Quand les Diatomées sont rangées, le cover est porté sur lả lame de platine et chauffé au rouge. L'enduit noireit, puis disparait entic̀rement, mais les frustules restent en place. Le eover refroidi est alors appliqué délicatement, les Diatomées en dessous; sur une lamé porte-objet où l'on a tracé une ou deux ecllules eoncentriques avec du bitume. Les eellules doivent ètre bien sèehes et préparées au moins trois semaines d'avance. La préparation est ehauffée trẻs légèrement pour obtenir l'adhérence que l'on aide ensuite en appuyant sur le cover. Enfin, la préparation est fermée par un cercle de bitume sur les bords du eover.

M. A. Truan emploie comme enduit, pour fixer les Diatomées sur le eover, le mélange suivant:

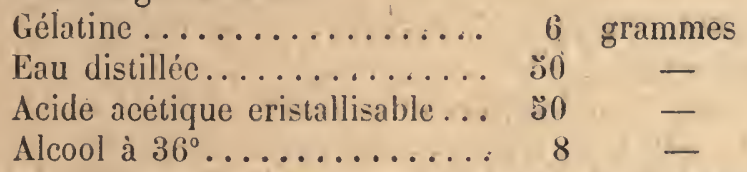

On met la gélatine et l'eau dans un flaeon de 200 grammes de eapacité et on les laisse en contaet jusqu'à ce que la gélatine n'absorbe plus d'eau; puis, on plonge le flacon dans l'cau ehaude, en agitant, pour eompléter la dissolution de la gélatine, et on ajoute l'aeide acétique et l'alcool. On replace le flacon dans l'eau ehaude pour rendre la solution plus liquide et on la filtre sur le papier en rejetant sur le filtre les premières parties qui passent. Puis, on eonservè le liquide dans un flacon bien bouché (1).

On se sert de cette solution gélatinée en y plongeant une aiguille que l'on passe à plat sur le couvre-objet bien propre et on laisse

(1) A. Truax y Luard, Ensayo sobre la Sinopsis de las Diatomeas de Asturias, Madrid, 1884. 
sécher sous une cloche. On peut ainsi préparer d'avance plusieurs covers.

On range les Diatomées sur un couvre-objet ainsi préparé, comme nous l'avons dit, en ramollissant l'enduit avec l'haleine, et l’on termine le montage comme nous l'avons indiqué.

\section{Préparation dans la naphtaline monobromée.}

La naphtaline monobromée a été quelquefois employée par M. Möller comme milieu à haut indice pour le montage des Diatomées. Son indice de réfraction est, en effet, représenté par le nombre 1,63 , celui du baume du Canada n'étant que $1, \mathfrak{2} 2$.

Voici comment M. A. Truan conseille de l'employer (1) :

On commence par préparer des cellules sur des porte-oljeis avec une solution épaisse de gomme laque dans l'alcool. Il faut les faire assez profondes et un peu plus larges que le cover dont elles doiven dépasser légèrement les bords. On les laisse sécher un jour ou deux, puis on les use en les frottant sur une pierre à aiguiser bien plane, de manière à ce que leurs bords soient eux-mêmes parfaitement plans.

Les Diatomées ayant été rangées sur un cover enduit de gélatine, comme nous l'avons indiqué, on dépose sur celui-ci une goutte de naphtaline monobromée et on laisse sécher à l'abri de la poussière.

Puis on met une goutte de naphtaline monobromée au milieu d'une cellule tracée à la gomme laque sur un porte-objets, et on retourne sur celle-ci le cover portant les Diatomées, sans emprisonner des bulles d'air. On appuie légèrement sur la lamelle, et on enlève l'excès de liquide avec du papier buvard, en essuyant, au besoin, avec un petit linge humide. Si l'adhérence entre le cover et les bords de la cellule ne s'est pas produite, on ramollit ceux-ci en passant tout autour une aiguille chauffée, et l'on appuie sur la lamelle en épongeant le liquide excédant.

L'adhérence ainsi obtenue, on laisse sécher et on ferme la préparation en traçant sur les bords de la cellule un cercle de gomme laque qui recouvre un peu la circonférence du cover. On termine, si l'on veut, en traçant par dessus le cercle de gomme laque un cadre au bitume de Judée ou autre vernis.

Au lieu de gélatine pour fixer les Diatomées sur le cover, II A. Truan indique un mélange d'albumine et d'ammoniaque obtenu en battant un blanc d'œuf, sans aucune trace de jaune, avec son 
poids d'eau distillée et ¿̇ grammes d'ammoniaque pure, jusqu'à ce que tout soit transformé en écume. On laisse reposer et on filtre plusieurs fois sur le papier.

On prépare les courre-objets avec cette solution comme avec la gélatine, et on y fixe les Diatomées en ramollissant l'enduit sec avec l'halcine. Quand on a terminé, on place le cover ainsi chargé sur la plaque métallique et l'on chauffe pour coaguler l'albumine. On opère ensuite comme il a été dit plus haut.

Enfin, on peut employer comme enduit une dissolution faible de gomme laque blanche dans l'alcool absolu. On l'emploie comme la gélatine, sauf que pour la ramollir, au moment où l'on y dépose chaque Diatomée, on ne se sert pas de l'haleine, mais de la chaleur en plaçant le cover sur la plaque métallique chaude, ou en approchant de la surface résineuse une tige de métal chauffée. Puis, le rangement des Diatomées achevé, on chauffe le cover sur la plaque, métallique de manière à fondre la gomme laque qui, sans cette précaution, serait attaquéc par la naphtaline monobromée. 


\section{VII}

\section{MOYENS D'ÉTUDE}

\section{\$ 1. - LES MICROSCOPES}

On dit souventqu'on peut faire de bonne besogne avec de mauvais outils et de bonnes obscrvations avec un mauvais microscope; la chose est possible, en effet, comme le prourent les admirables découvertes que les Leeuwenhoek, les Swammerdam, les Malpighi surent faire avec les instruments grossiers qu'ils possédaient et fabricaient le plus souvent eux-mèmes en fondant à la chaleur un globule de verre. Mais il n'est pas donné à tout le monde d'égaler ces illustres fondateurs de la micrographie, et il est probable que les savants de nos jours se trouveraient fort empèchés s'ils n'avaient que ces instruments rudimentaires pour approfondir les recherches qu'ont commencées leurs célèbres devanciers.

L'étude des Diatomées est particulièrement une de celles qui exige l'emploi des meilleurs instruments, non seulement au point de vue optique, mais encore sous le rapport de la construction mécanique. C'est mème cette nécessité, pour le diatomiste, de mettre en œuvre les moyens d'observation les plus parfaits qui a été la principale cause des perfectionnements incessants que les opticiens ont apportés, depuis le commencement de ce sic̀cle, à la construction des microscopes et surtout des objectifs.

Il ne s'agit plus, en effet, de reconnaitre plus ou moins exactement, comme au temps de Joblot, la forme et l'aspect d'une Diatomée ; chaque frustule se présente maintenant au micrographe comme une énigme qu'il s'agit de déchiffrer : il offre, dans son intérieur, une structure particulière et compliquée dont il faut déterminer les parties diverses et les dispositions variées, et, sur ses faces, des caractères, des dessins, des ciselures qu'il faut pouvoir reconnaitre, caractériser, compter. - Et tout cela, dans l'espace de quclques millièmes de millimètre; si bien que ces détails, si extraordinairement petits 
qu ils atteignent la fraction de longueur d'onde que l'ceil humain ne peut plus distinguer et qui marque la limite de la visibilité, il faut souvent, rien que pour les entrevoir, accumuler toutes les ressources et tous les perfectionnements de l'optique moderne.

Aussi, quiconque voudra faire des Diatomées une étude sérieuse et trouver dans cette étude toutes les jouissances qu'elle peut procurer à un naturaliste devra se munir du meilleur microscope possible accompagné des objectifs les plus parfaits.

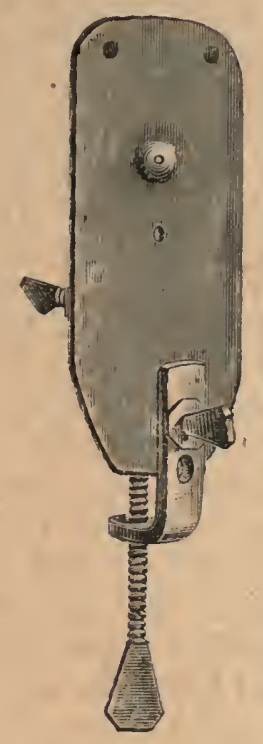

$a$

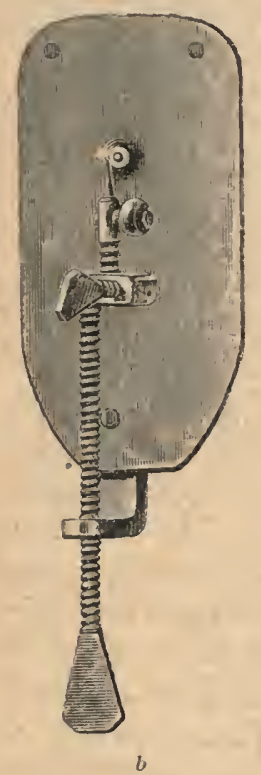

Fig. 75. - Microscope de Leeuwenhoek (1673)

$a$ : Face antérieure. - $b$ : Face postérieure.

La lentille est comprise dans une concavité entre déux lames d'argent fixées l'une à l'autre par trois rivets; la platine, mue par une vis verticale et une vis horizontale, porte une pointe pour placer l'objet, laquelle pointe est mobile aussi, à l'aide d'un bouton (1).

Les bons instruments sont maintenant nombreux et nous n'en décrirons que quelques-uns qui méritent pour les diatomistes une mention particulière et que nous leur recommandons tout spécialement.

L'un des meilleurs est.le microscope grand modèle de la maison Hartnack et Prazmowski, de Paris, maison qui appartient aujourd'hui à MM. Bézu, Hausser et $C^{\text {ic }}$, sucecsseurs de Prazmowski.

(1) Vuir Journal de Micrographie, 1×87, p. 337 . 
Co modèle est depuis longtemps connu de tous les micrograplies, et e'cst certainement l'instrument le plus sérieux qui se construise en France; aussi a-t-il été imité partout. Voici, en quelques mots le principe sur lequel il est établi (Fig. 76).

Le microscope cst, bien entendu, à inclinaison, et la platine, carréc arec les angles mousses, large, garnie de glace ou d'ébonite, est à tourbillon, c'est-á-dire qu'elle peut tourner autour du centre optique dans un plan perpendiculaire à l'are, quelle que soit l'inclinaison qu'on a donnée à cet axe. Dans ce mouvement, elle cmporte avec elle tout le corps de l'instrument, la colonne du mouvement lent et le tube, qui viennent ainsi prendre toutes les positions autour du centre optique, le pied et le miroir restant sculs fixes.

Ce mourement s'exécute arce une précision extrème et il est construit arec une grande solidité, de manière à ce que le centrage cxact des pièces soit rigoureusement.maintenu.

Du reste, ccttc solidité, cette perfection et cette garantie du centrage constituent le but principal qui a été recherché dans la construction de l'instrument tout entier.

C'est en vue de ce résultat que la crémaillère à l'aide de laquelle le tube s'élève et s'abaissc, par ce qu'on appclle lc mouvement rapide, est montće sur le tube lui-même au licu d'ètre fixée sur un cylindre ou coulant fendu dans sa longueur et dans lequel te tube glisse à.frottcment, comme cela est prátiqué par beaucoup de constructeurs. Ce système du coulant, qui permet de faire mouvoir le tube à la main et mème de l'enlever tout à fait, est assez commode, mais il nuiı considérablement à la solidité, en mème temps qu'il rend impossible la conservation du centrage. Au bout de fort peu de temps, lc tube finit toujours par ne plus se mouvoir droit, le coulant se fatigue et l'axe optique se déplace toutes les fois qu'on fait tourner le tube sur lui-mème.

C'est eneore en vue de la solidité et de la fixité de l'axe optique que la colonne contenant la vis micrométrique de ce qu'on appellc lic mouvement lent se meut sur un prisme triangulaire comme axc ct non sur un cylindre. On comprend que, de cette manière, il n'y a aucun déplacement latéral du corps du microscope ni aucun ballottenent, comme il s'en produit toujours plus ou moins quand le mouvement lent résulte du glissement d'un cylindre creux sur un cylindre plein. Ici, l'àme prismatique de la colonne s'applique par tous les points de sa surface et par toute la longueur de ses arêtes sur le prisme fixe qu'elle emboite. Et, pour assurer encore l'exacte application des surfaces, l'un des côtés du prisme servant d'axe est évidé ct le vide est rempli par une lame d'acier bombéc en dehors, faisant ressort, ct dont l'élasticité établit un serrage des surfaces on contact les unes sur les autres. 
Sous la platine était naguère plaeé uno sorte de tiroir latéral, glissant dans des coulisses, et qui pouvait être amené sur le côté pour recevoir les divers diaphragmes et les appareils spéciaux d'éelairage. Il suffisait alors de le repousser sous la platine, où sa position était déterminée par un bouton d'arrèt, pour que ces appareils tombassent exaetement dans l'axe optique. Ce système a été remplacé aujourd'hui par un levier excentrique qui vient se développer sur le còté, ce qui remplit le mème but d'une inanière plus commode.

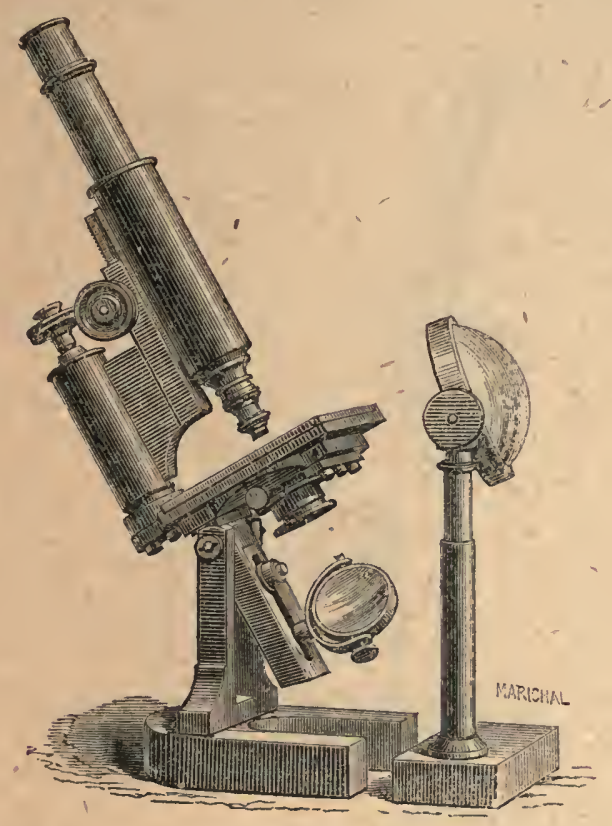

Fig. 76. - Microscope, grand modèle, de Hartnack et Prazmowski (Bézu, Hausser et $\mathrm{C}^{\mathrm{i} e}$ ).

Tels sont les principaux détails de eonstruetion de cet instrument. Il est, en effet, inutile d'ajouter qu'il est monté sur un pied lourd, en fer à cheval, garni de peau par dessous, que Ie miroir, plan d'un eóté, eoneave de l'autre, mobile dans une glissière et sur un bras artieulé, peut donner tous les dégrés d'obliquité dans l'éelairage. Enfin, le tube à tirage a 12 eentimètres de haut quand il n'est pas développé, 16 centimètres quand il cst entièrement tiré.

On voit que cet instrument qui; prêt à l'emploi, mesure, au plus, 30 centimètres de haut dans la position vertieale et est naturellemeut 
moins haut encore quand il est incliné, bien que très complet et pouvant recevoir tous les accessoires que nécessitent les recherches les plus délicates, est, néanmoins, de forme ét de dimensions très commodes, nullement encombrant, très maniable, et se présente comme un instrument de travail des mieux appropriés, des plus sùrs et ayant, de plus, cet arantage de n'exiger jamais de réparations.

Ce modèle a été reproduit dans des proportions un peu moindres mais arec les mêmes dispositions (Fig. 7i), par MII. Bézu, Hausser et $C^{\text {ie }}$, qui ont mème aménagé un petit modèle fort bien compris,

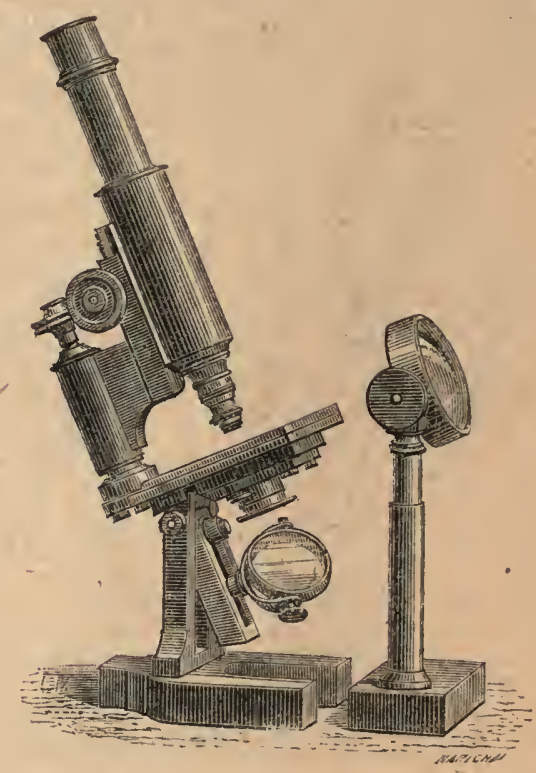

Fig. 77. - Microscope, moyen modèle, de Hartnack et Prazmowski (Bézu, Hausser et $\mathrm{C}^{\mathrm{io}}$ ).

mais dont la platine n'est plus à rotation. En revanche la sousplatine a été modifiée; elle est portée par une crémaillère qui permet d'élever et d'abaisser facilement les diaphragmes et appareils d'éclairage malgré le peu d'élévation de la platine (Fig. 78).

Ces instruments sont munis d'un adapteur qui remplace la vis pour fixer les objectifs à l'extrémité du tube.

Mais il est un modèle que nous croyons pouvoir recommander d'une manière toute spéciale, c'est le microscope que MIr. Bézu, Hausser et Gie construisent particulièrement pour l'étude des bactéries.

Bien qu'établi, comme nous le disons, en vue de la bactériologie. 
cet instrument se prète admirablement aussi à l'étude des Diatomées, grâce à diverses modifications que les constructeurs ont apportées à la forme et au mécanisme du modèle généralement adopté en France et en Allemagne et que nous avons appelé jadis modèle continental par opposition aux modèles anglais et-américains construits ordinairement sur un autre principe.

Cet instrument, en effet, est à platine tournante, mais non plus pall ce procédé qui consiste à faire tourner, avec la platine, tout le corps du microscope, la colonne, le tube, l'oculaire, tandis que le pied scul reste fixe. Dans le modèle dont nous nous occu-

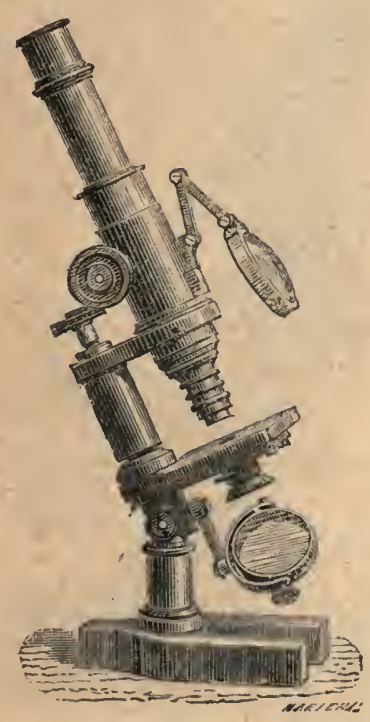

Fig. 78. -.. Microscope, petit modèle, de MM. Bézu, Hausser et Cie.

pons, les constructeurs ont adopté le système anglais que, depuis de longues années, nous ne cessions de recommander : la platine, circulaire, tourne scule sur son support, autour de son centre, c'est à dire autour de l'axe optique, le nicroscope entier, corps, tube et oculaire, restant fixes, Cette platine, garnie de glace, qui peut d'ailleurs recevoir un système à mouvements rectangulaires, mesure 9 centimètres $1 / 2$ de diamètre; clle a done des dimensions suffisantes pour toute espèce de travail. D'ailleurs, sa surface supérieure ne se trouve qu'à 12 centimètres $1 / 2$ au dessus du plan de la table, ce qui permet d'y travailler sans fatigue pour la main, ni pour le bras. Néanmoins, elle est munie, en dessous, d'un condensateur d'Abbé porté par un excentrique à l'aide duquel on peut amener l'apparei! 
dans l'axe optique ou le rejeter sur le còté. Cet appareil peut ètre enleré et remplacé par de simples diaphragmes ou un appareil de polarisation.

L'instrument est construit sur les mèmes principes que les précédents, avec une extrême solidité et une précision rigoureuse dans le mécanisme: colonne du mourement lent montée à prisme, crémaillère portée sur le tube lui-mème, pour maintenir l'exactitude du centrage. La vis micrométrique du mouvement lent a exactement $1 / 4$ de millimètre de pas, et le bouton qui la surmonte est divisé sur

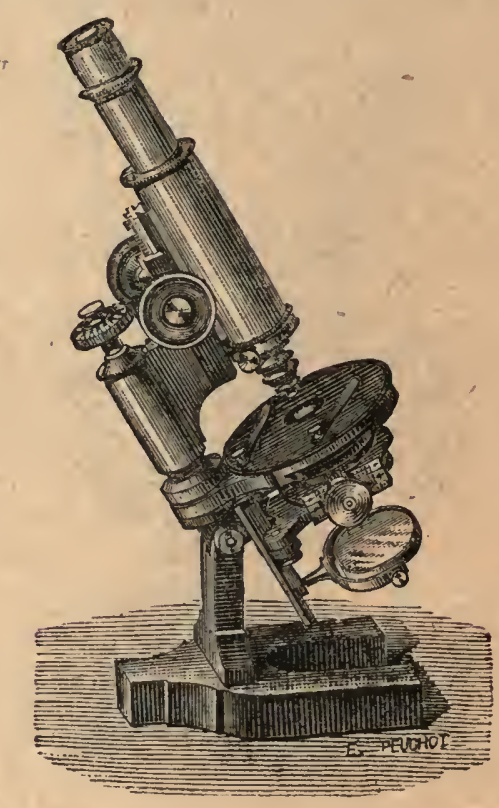

Fig. 79. - Microscope bactériologique de MM. Bézu, Hausser et $\mathrm{C}^{\mathrm{ie}}$.

ses bords en 50 parties, ce qui permet de mesurer un mouvement vertical du tube de $\frac{1}{200}$ de millimètre. Et mème, gràce à l'index fixe placé devant la tète de vis, on peut apprécier facilement un déplacement d'une demi-division, c'est à dire un mouvement vertical de 1/400 de millimètre.

Cet instrument est, comme les précédents, muni d'un adapteur. pour fixer les objectifs.

Le tube, à tirage, mesure 13 centimètres sans le tirage, et 20 centimètres quand celui-ci est entièrement développé.

Le microscope, porté sur un pied lourd, en fer à cheval n'a, prêt 
à l'emploi, dans sa plus grande dimension et dans la position verticale, que 40 eentimètres de haut. C'est done un instrument fort commode et très maniable; aussi, malgré son tịtre de « bactériologique, " nons n'hésitons pas à le considérer eomme le microscope spécial des diatomologistes et e'est comme tel que nous le reeommandons.

Nous ne nous attarderons pas à décrire d'autres mieroscopes de construction française, et nous nous bornerons à ceux dont nous avons parlé, pensant que l'on ne saurait trouver mieux. Quant aux

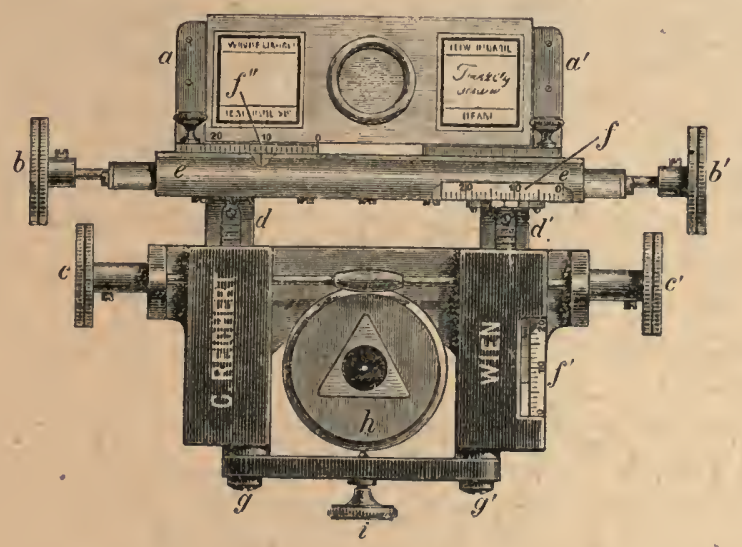

Fig. 80. - Platine mobile à mouvements rectanæulaires, de M. C. Reichert, de Vienne). .

instruments de construction étrangère, nous en dirons peu de chose.

Les mieroscopes anglais et américains, ces dernicrs surtout, et particulièrement les grands modèles, sont le plus sourent des ehefsd'œúvre de mécanisme; mais, outre que leur prix, extrèmement élevé, ne les rend guère abordables qu'à quelques privilégiés, ils sont réellement un peu encombrants, avec leurs proportions monumentales. Aussi, ce sont plutôt des instruments d'apparat, construíts pour l'exhibition, les conférences et les " soirées 》 que des instruments de recherches créés en vue du laboratoire ou du cabinet de travail. Les conśtrueteurs anglais et américains, fort habiles, nous le reconnaissons, fort ingénicux, et auxquels nous sommes ecrtainement redevables de nombreuses dispositions utiles, ont, à notre avis, le tort de faire un peu trop grand (pendant que nous faisons 
quelquefois trop petit). Tcl objcctif anglais, par exemple, représente réellement un petit obus qu'il pourrait ètre dangereux de se laisser tomber sur le pied, tandis que l'objectif français correspondant est plus petit qu'un dè à coudre. Il y a donc, dans l'instrument anglais, une dépense de matière et de travail qui parait inutile, mais que, naturellement, l'acheteur doit payer.

Quant aux petits modèles de microscopes anglais ou américains, comme ils ne possèdent plus, en général, les dispositions des grands modèles et que comme construction ils sont, le plus souvent, loin de valoir les nôtres, il n'y a aúcun intérèt à s'adresser à eux.

Ajoutons que tous ces instruments, quelqu'admirables qu'ils puissent être, - et ils le sont souvent - ont un défaut commun et sérieux : les systèmes, à lévier ou autres, employés pour lc mouvement lent, sont très fragiles et se dérangent trop fréquemment.

Quant aux microscopes allemands, il sont en général établis sur les mèmes principes que les nôtres. Les modèles actuels, en effet, datent de l'allenand Oberhæuser qui, renu s'établir cn France jadis, y subit l'influence des idées alors régnantcs de Ch. Chev́alier, idées mises en pratique, d'une part, par Nachet pèrc et, de l'autrc, par l'allemand Hartnack, successcur d'Oberhæuser à Paris. C'est de cette combinaison qu'est né le modèle continental. En 1870, Hartnack qui, dans sa haine pour la France, voulait détruire_sa maison, retourna à Berlin, laissant pour seul successeur à Paris son associć, le savant émigré polonais A. Prazmowski, l'ancicn professeur de physique à l'Université de Varsovie et dirceteur de l'Obserratoire de cette villc. Condanné à mort par les Russes, ce patriote, français de cœur, était venu à Paris où, pendant de longues annécs, il a travaillé aux progrès de la micrographic. C'est lui qui a su donner à la maison dont il avait pris les rènes la direction si éminemment scientifique qui en a fait la valeur. Cette maison, nous l'avons dit, est aujourd'hui dignement continuée, et l'on peut dire rajeunie, par MM. Bézu et Hausser dont nous avons déjà parlé.

Ce court historique montre que le microscope désigné par nous autrefois sous le nom, adopté maintenant, de microscope continental, provient cn quelque sorte de la combinaison des idécs françaises et allemandes. Il en résulte qu'aujourd'hui c'est cncore, en Allemagne comme en France, le modèle classique. Seulement, en Allemagne, il est moins bien construit, sous le rapport mécanique, que dans notre pays. Les prix de vente sont peut-êtrc inférieurs de quelques francs chez certains fabricants, mais les instruments sont de facture très notablement inférieurc, et, dans l'étude des Diatomées, la perfection du mécanisme s'impose absolument.

C'est pourquoi nóus pensons que les micrographes de notre pays 
n'ont aucune raison d'aller chercher des microscopes en Allemagne. Nous verrons plus loin ce qu'il y a à dire à propos des objectifs.

Toutefois, si nous n'avons qu'une très médiocre estime pour les microscopes saxons, bavarois, prussiens, etc., nous derons faire

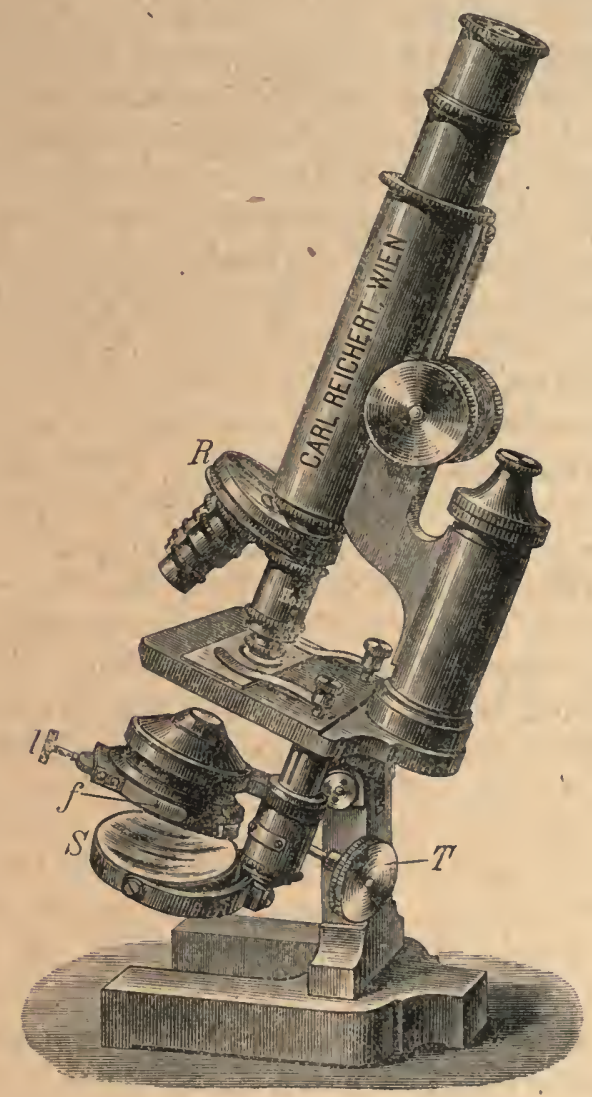

Fig. 81. - Microscope bactériologique de M. C. Reichert, de Vienne.

R. Revolver - S. Miroir. - T. Crómaillère du condenseur Abbé. - $f$. Porte-diaphragmes du condenseur. $-l$. Bouton mouvant le porte-diaphragmes.

exception pour ceux que construit M. Carl Reichert, de Vienne (Autriche), et qui sont tout à fait recommandables.

Cette maison Viennoise est dejjà fort ancienne, mais depuis une dizaine d'années, surtout, ellc a pris un rang très important dans la 
construction des microscopes; c'est à l'Exposition Universelle de 1878 que nous avons examiné pour la premièrc fois ses instruments déjà fort rcmarquables et qui nous ont été préscntés par le professeur Von Fleischl.

Depuis lors, elle a fait de grands et inccssants progrès et ses microscopes ainsi que ses autres appareils d'optique peuvent ètre comptés parmi les meilleurs.

Nous citerons seulement son grand modèlc $n^{\circ}$ I dont la platine est à tourbillon, c'est-à-dire tournc avec lc corps du microscope, et le modèle $n^{\circ}$ II $b$ dont la platinc est fixe, cc qui n'a pas d'inconvénicnt notablc, l'instrument étant muni (comme le grand modèlc, d'ailleurs) d'un éclairage d'Abbé porté sur une tige excentrique.

Ces microscopes sont construits sur les principes que nous avons indiqués plus haut, montés à prisme, avec la crémaillère fixéc dircctement sur le tube ct la tête de la vis micrométrique, divisée, tournant devant un index fixe.

Ajoutons que M. C. Reichert a invénté un chariot mobile, à mouvements rectangulaires, qui sc place à volonté sur la platine, à l'aide de crans d'arrêt et d'une vis de serrage prenant son point d'appui à la face postérieure de la colonne (Fig. 80).

Enfin, nous devons signaler, du mème constructeur, un microscope bactériologique, à platine carréc (pouvant recevoir le chariot mobile), non rotative, mais munie d'un éclairage du système $\mathrm{Abbé,}$ et qui est un fort bon instrument. La tète de la vis micrométrique est diviséc et le nombre des divisions dont clle tourne est indiqué par un index.

\section{§ 2. - LES OBJECTIFS}

Si la construction du microscope doit ètre parfaite pour l'étude des Diatomées, on conçoit de quelle importance doit être l'excellence des objectifs.

Nous ne pouvons faire ici la théorie de la construction des objectifs et de la formation des images microscopiques, nous rappcllerons sculement quc les objectifs, pour servir utilement à l'obscrvation des fins détails de structure des Diatomécs, doivent êtrc doués d'un grand pouvoir de définition et de résolution, c'est-à-dire fournir des images dont les contours et les linéaments soient cxtrêmement nets ct dont les parties soient bien dessinées et distinctement séparées.

Quant au pouvoir de pénétration, il est presque toujours suffisant pour l'cxamen de ces petits corps. On sait que ce 'qu'on appelle 
le pouvo ir pénétranl est la propriété qué possèdent certains objectifs de laisser voir à la fois, sans changement de la mise au point, un certain espace dans la profondeur de l'objet. - Cette propriété est généralement incompatible avec une bonne définition et une fine résolution. Mais, comme l'a, d'aillcurs, très bien fait remarquer Helmholz, ce pouvoir pénétrant n'est pas absolument une propriété de l'objectif, il dépend aussi pour une bonne part de l'œil de l'observatcur dont la faculté d'accommodation est plus ou moins grande et qui peut distinguer à la fois des points plus ou moins éloignés au dessus et au dessous du foyer mathématique.

Pour l'obscrvation des Diatomées, il faut des objectifs ayant les plus grands pouvoirs définissant et résolvant; ce sont, d'ailleurs, les plus parfaits et les sculs qui permettent de juger avec certitude l'ordre de superposition des divers points dans l'épaisseur de l'objet. Quant à la pénétration, elle est ici peu utile et il ne faut rechercher que eclle qui subsiste dans l'objectif dont la résolution est aussi parfaite que possible, pénétration aidée de la faculté d'accommodation que possède l'œil de l'observateur.

Par conséquent, les objectifs doivent avoir la plus grande ouverture possible.

Quant à l'ouverture, on sait qu'on appelait autrefois ouverture angulaire d'un oljectif l'angle du plus grand còne de rayons qui pouvait entrer dans l'objectif. On comprend que, quelle que soit la combinaison optique qu'on ait imaginée pour admettre et faire concourir à la formation de l'image le plus large cône de rayons, l'objectif agissant dans l'air comme milieu réfringent extéricur, il y avait toujours un moment où les rayons extrèmes ne pouvaient plus entrer dans l'objectif parce que leur obliquité atteignait l'angle limite au delà duquel les rayons ne pénètrent plus, en se réfractant, de l'air dans le verre, mais se réfléchissent totalement à la surface de celui-ci. Cet angle limité cst de $41^{\circ} 49^{\prime}$.

C'est précisément cette impossibilité de faire entrer un très large còne de rayons dans les objectifs observant les objets à travers une couche d'air, objectifs dits à sec, qui a suggéré à Amici l'idée de remplacer la couche d'air, interposéc entre l'objet et la face inférieure de la lentille objective, par un milicu dont l'indice de réfraction diffère moins, que celui de l'air de l'indice de réfraction du verre dans lequel sont taillées les lentilles. Il a essayé plusieurs liquides, et s'est arrêté à l'eau, créant ainsi cc que l'on a appelé des objectifs_à immersion.

Ces objectifs, en raison du peu de différence entre les indices de l'eau et du verre, ont permis d'agrandir considérablement l'ouverture angulaire, si bien que le conne de lumière admise avait un angle qui approchait de $180^{\circ}$. On sait tous les scrvices qu'ont rendus et 
que rendent encore journellement ces objectifs à immersion dans l'eau, dont la construction a rendu célèbres les noms de Hartnack et Prazmors ski, de Tolles, de Powell et Lealand et de quelques autres opticiens habiles.

Néanmoins, l'indice de réfraction de l'eau, exprimé par le chiffre 1,33, cclui de l'air étant 1, présente encore une différence notable àvec celui du verre des objectifs (croun glass), qui est en moyenne 1,328 . C'est pour cela qu'Amici avait pensé à employer la glycérine et certaines essences, comme l'essence d'anis, dont l'indice se rapproche davantage de celui du verre. S'il n'avait pas renoncé à l'emploi de ces liquides, comme peu pratique, il aurait inventé ce qu'on appclle aujourd'hui les objectifs à immersion homogène avec lesquels on use, pour l'immersion, de liquides qui ont sensiblement le mème indice que le verre. Cette appellation, assez mal choisic, veut exprimer que la lumière se meut ainsi dans un milieu, verre et liquide, qui est optiquement homogène puisqu'il a partout le mème indice de réfraction.

On sait que c'est M. C. Zeiss, d'léna qui, sur les calculs du professeur E. Abbé, et les expériences de M. R. Stephenson, de Londres, a construit les premiers objectifs à immersion homogène, qui fonetionnent avec l'huile essentielle de bois de cèdre, dont l'indice est $1, \because 1 \%$. Néanmoins, ce n’est pas là une découverte ni de M. Zeiss, ni de MI. Abbé, ni de M. Stephenson : c'est la réalisation, conduite à bonne fin, de l'invention d'Amici, laquelle date de 1844. Comme nous l'avons dit, Amici, inventeur de l'immersion, apris avoir essayé une'série de liquides et entr'autres plusieurs huiles essentielles, s'est arrêtẻ à l'eau, liquide facile à trouver partout, dont l'indice est connu et ne varie pas. Ce progrès était immense et suffisait aux besoins des micrographes de cette époque.

C'est aussi M. E. Abbé qui a remplacé la mesure de l'ouverture angulaire des objectifs, laquelle était souvent illusoire, presque toujours incxacte et d'ailleurs sujette à des interprétations crronécs, par unc autre mesure appeléc ouverture numérique.

Cette quantité est représentée par l'expression :

$$
0=n \sin u
$$

dans laquelle $n$ représente l'indice de réfraction du milieu ambiant, air, eau, huile, et $u$ le demi-angle d'incidence des rayons extrèmes.

Ainsi, un objectif à sec qui aurait lc maximum d'ouverture angulaire, $180^{\circ}$, aurait pour ouverture numérique 1 . En effet, dans la formule $0=n \sin u$, l'indice $n$ de l'air est 1 , et l'angle $u$ est la moitié de $180^{\circ}$, c'est-à-dire $90^{\circ}$ dont le sinus est 1 . La formule numérique devient donc $0=1 \times 1=1$.

On voit ainsi qu'à cet objectif à sec, d'ouverture angulaire maximun, $180^{\circ}$, et d'ouverture numérique $=1$, correspond, comme 
puissance optique, un objectif à eau de $9 \overline{7}^{\circ}$ seulement d'ouverture angulaire. Car le sinus de $48^{\circ} 1 / 2$, moitié de $97^{\circ}$, est sensiblement 0,7032 , qui, multiplié par 1,33, indice de l'eau, donne pour valeur de l'ouverture numérique : 1 .

Et les mèmes effets optiques scraicnt produits par un objectif a immersion homogène, à huile, qui n'aurait que $82^{\circ}$ d'ourerture angulaire, car dans la formule $0=n \sin u$, l“indice $n$ est $1,32 \mathrm{et}$ $\sin u$ ou $\sin 41^{\circ}$, est sensiblement 0.638 . D'où $0=1,32 \times 0,6.38=1$.

On verrait ainsi quà un objectif à sec qui aurait $128^{\circ}$ (dans l'air) d'ouverture angulaire, correspondrait un objectif à immersion dans l'eau qui n'aurait que $83^{\circ}$, parce que tous deux ont la mème ouverture numérique $=0,90$. En effet, la formule donne.pour l'ouverture numérique du premier $0=1 \times \sin 64^{\circ}=1 \times 0,90=0,90 ; \mathrm{et}$ pour célle du sceond: $0=1,33 \times \sin 42^{\circ} 1 / 2=1,33, \times 68=0,90$.

De mème encore, un objectif à immersion lomogène $(n=1, ; 02)$ qui n'aurait que $92^{\circ}$ d'ouverture angulaire, correspondrait à un objectif à immersion dans l'eau $(n=1,33)$, de $112 \circ$ d'ouverture angulaire, car tous deux auraient pour nuverture numérique : 1,10 comme il est facile de le voir par le calcul.

On reconnait enfin que si, comme nous l'avons vu plus haut, un objectif à eau dont l'ouverture angulaire est de $97^{\circ}$ et l'ouverture numérique 1 , et un objectif homogène de $82^{\circ}$ d'ouverture angulaire ou d'ouverture numérique égale aussi à 1, correspondent à un whjectif à sec de $180^{\circ}$ d'ouverture angulaire avee ouvęture numérifue égale à 1 , tous les objectifs à eau qui ont une ouverture angulaire plus grande que $97^{\circ}$ et tous les objectifs homogènes dont l'ouverture angulaire est plus grande que $82^{\circ}$ correspondent à des objectifs à air dont l'ouverture angulaire serait plus grande que $180^{\circ}$.

Or ce résultat parait absurde. On ne comprend plus un objectif dont l'angle d'ouverture serait plus grand que $180^{\circ}$, ce qui, en eflet, est irréalisable dans la pratique avec les objectifs à see. C'est précisément ces considérations qui ont excité de longues et vives discussions entre les nicrographes sur ee qu'on a appelé la question de l'ouverture. Et c'est aussi une des raisons qui ont amené le prof. Abbé à abandonner la notion de l'ouverture angulaire, laquelle n'a plus de sens à une certaine limite, pour la remplaecr par celle de l'ouverture numérique qui s'applique à tous les cas.

II. Abbé a inventé un instrument, dit apertomètre, qui permet de mesurer l'ouverture numérique des objectifs (1).

On eomprend que nous ne pouvons entrer ici dans les détails d'optique géométrique que soulève eette question de l'ouverture 
numérique et nous renverrons pour plus de développement sur ce sujet aux ouvrages spéciaux (1). Nous ajouterons sculement que, dans la pratique, l'ouverture numérique la plus considérable des

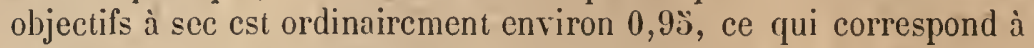
ce qu'on appelait naguèrc une ouverture angulairc de $140^{\circ}$. L'ouverture numérique des objectifs à immersion dans l'cau cst ordinaircment de 1,10 å 1,20 , et celle des objectifs à immersion lıomogène va à peu près de 1,20 à 1,40 .

Pour que la définition et la résolution soient au maximum dc pouvoir, il faut que l'ouvcrture numérique de l'objectif soit représcntéc par un chiffre élevé. En cffet, puisque cette quantité est le produit de l'indice de réfraction du milicu dans lequel fonctionne l'objectif par le sinus du 1/2 angle des rayons incidents extrèmes, dans un même milieu, l'indice ne changeant pas, ce produit est d'autant plus grand que le sinus est plus grand, c'est-à-dire que l'angle lui-mêmc des rayons extrèmes est plus grand.

Et il faut que cet angle soit grand pour que l'objectif admette et supcrpose dans l'image un plus grand nombre des spectres de diffractions que forme la lumière en traversant l'objet à fine structure que l'on cximine.

On sait aussi, en effet, que le professeur Abbé a établi, sur des expériences probantes, une nouvelle théoric de la formation des images dans le microscope, théorie que nous ne pouvons exposer ici, mais que nous résumerons en quelques mots (2).

On admettait autrefois que, dans lc microscope, l'oljectif avait pour fonction de former, par les simples lois de la réfraction dans les lentilles, une image réelle, renversée et agrandie de l'objet, image que recevait l'oculairc et qu'il agrandissait de noureau, fonctionnant comme une loupe.

D’après M. Abbé, « le prenier acte de l'objectif consiste plutòt dans la formation d'une image virtuelle à distance infinie avec des rayons parallèles. Le second acte comprend la réfraction ultime à travers la face postérieure de l'objectif et les autrcs réfractions qui se produisent dans l'oculaire, réfractions par suite desquelles

(1) On doit avouer que les ouvrages où ces questions sont traitées d'une manière intelligible pour le plus grand nombre des lecteurs sont fort rares. C'est une lacune que nous avous essayé de combler dans notre petit livre : Théorie du microscope d'après les idées actuelles (sous presse).

(2) E. ÁввÉ. Beitrcege zur Theorie des Mikroskops, etc. (Arch. für mikr. Anatomie, IX, 1873, p. 413, et Monthly micr. Journal, XIV, 1875.)

Hецмноцт. Die theoretische Grenze für die Leistungs foehliigkeit des mikroskops, (Ann. de Poggendorf, 1874, Jubelband, et Month. Micr. Journ. XVI, 1876).

E. GiLrar. Inleiding to het gebruik van den Microscoop. Leideǹ, 1885. 
l'image se forme, à la distance de la vision distinete, avec des rayons visuels divergents. Le premier acte répond simplement à la fonetion d'une lentille grossissante ordinaire, tandis que le second répond i la fonction d'un téleścope (avec un objectif de petite ouverture) auquel sert d'objet l'image virtuelle, à distance infinie, formée par le premier processus.»

C'est ainsi que se produit, autrement qu'on l'exposait, mais toujours par les lois de la réfraetion, l'image générale donnant li forme et les contours de l'objet. Mais pour la production, dans l'image, des fins détails de structure de l'objet, interviennent des phénomènes de diffraction, analogues à cenx qui se produisent dans les réseaux.

On appelle réseau une surface présentant des londes ou des stries extrèmement serrées, alternativement opaques et transparentes, telles qu'on les produit, par exemple, en traçant sur une lame de verre, avec un diamant, une série de raies très rapprochées, comme cela se fait dans la fabrication des micromètres ; les raies dépolies sont opaques, tandis que les espaces entre les raies sont transparents. Si l'on regarde un point lumineux, comme la flamme d'une bougie, à travers ce réseau, on ne voit pas seulement une flamme, mais une série d'images de la flanme, de chaque cité de celle-ri, sur une ligne perpendiculaire à la direction des stries du réscau. Et ces images sont étalćes, colorées des couleurs du spectre, d'autant plus étalées et d'autant plus éloignées les unes des autres que les stries du réseau sont plus rapproeliées. Ce sont des spectres de diffiaction.

\subsection{4:}

lig. 82. - Images de diffraction d'une flamme de bougrie vue à travers un réseau.

Or, pour qu'un objectif tournisse l'image des fins détails d'un objet, stries, ponetuations, - telles qu'il s'en trouve, par exemple, sur les Diatomées - il faut qu'il ait une ouverture suffisante pour

- receroir, et faire conconrir à la formation de l'image, le plus grand nombre de ees pinceanx diffractés par les détails de la fine structure. Et il faut admettre que plus l'oljectif ntilise de ces pinceaux diffractés, plus l'image qu'il fournit est conforme à l'oljet, plus, par conséquent, l’objectif ıéfinit et résout.

Le prof. Abbé, à l'aide d'un objet ou test préparé artificiellement, une petite lame d'argent portant des lignes gravées à la machine à 
diviser, test dont la structure est ainsi connue, a démontré que quand on arrête, avec des diaplıragmes convenablement établis, tels ou tels des spectres de diffraction produits par le test, on obtient des images absolument différentes et dont aucune ne représente la réalité. Quant au pinceau central qui donne une image par réfraction, il ne fournit que la représentation de la forme générale. des contours du test.

Il en résulte que le même objet, à fine structure, peut fournir des images différentes selon l'objectif avec lequel on l'examine et des images qui peurent ne pas représenter la réalité. De mème, des objets différents peurent, suivant l'objectif, donner des images semblables.

Et, en définitive, on n'est pas certain que l'image d'un objet vu avec le microscope représente cet objet tel qu'il est réellement (1).

Maintenant, peut-on espérer qu'en créant des objectifs à ouverture de plus en plus grande, admettant le plus grand nombre possible des spectres de diffraction fournis par un objet à structure très fine, on pourra arriver à distinguer, à résoudre, comme on dit, des détails de plus en plus fins de cette structure? - Non : il y a une limite à la visibilité. Ainsi, deux stries d'une Diatomée qui seraient à une distance l'une de l'autre plus petite que $\frac{1}{3636}$ de millimètre ne pourraient plus ètre résolues et l'œil ne les séparerait plus. Cette limite de la visibilité a été calculée par Helmholtz qui a trouvé qu'elle est donnée par la formule.

$$
s=\frac{\lambda}{2 \sin x}
$$

C'est-à-dire que $\varepsilon$, la plus petite distance résoluble avec le microscope, est ègale au quotient de la longueur d'onde $\lambda$ de la lumière dans laquelle on opère, divisée par le double du sinus de l'angle $\alpha$ que font les rayons incidents avec l'axe optique.

Ainsi, si l'on prend la partie la plus lumineuse du spectre, le jaune dans le voisinage du vert, la longueur d'onde $x$ est $0^{\mathrm{mm}}, 00035$;

(1) Consultez pour plus de détails, outre les ouvrages précédemment cités: Dippet. Das Mikroskop und seine Anwendung, 2 Ed. Braunschweig, 1882.

$\mathrm{FR}_{\mathrm{R}}$. CRISP. On the infuence of the diffraction in Microscopic vision (Journ. Quekett Micr. Club, 1878).

E. AввÉ. Ueber die Grensen der geometrischen Optick (Jen. Zeitsch. f. Naturoiss. T. XIV, sup. H. 1, jena, 1881).

$\mathrm{F}_{\mathrm{R}}$. CRISP. Notes on Aperture, microscopic vision, etc. (Journ. de Micro= graphie VI, 1882.

J. Pelletan. Théorie du Microscope, in $8^{\circ}, 1888$. 
si l'on suppose un angle d'ouverture maximum, $180^{\circ}, \lambda$ est égal à $90^{\circ}$ dont le sinus est 1 ; la formule donne alors les valeur's numériques suivantes :

$$
\varepsilon=\frac{2}{0.0005 \%}=0^{\mathrm{nm}}, 00027 \%=\frac{1}{3636} \text { de millim. }
$$

L'agrandissement de l'ouverture des objectifs ne saurait d'ailleur's ètre indéfini. S'il augmente, comme nous venons de le voir, le pouvoir résolvant des objectifs, il diminue la pénétration et réduit la distance frontale, e'est à dire la distanee entre l'objet et la faee inférieure de la lentille antérieure ou frontale, laquelle distance, dans les très forts grossissements, devient tellement petite que l'objectif est d'un emploi fort ineommode et qu'on a la plus grande difficulté à trouver des couvre-objets assez minces pour permettre la mise au point.

Du reste, il y a une relation foreée entre l'ouverture et le grossissement. On comprend qu'une grande ouverture, qui augmente le pouvoir résolvant, n'est utile que lorsqu'elle est aecompagnée d'un grossissement suffisant. Et réciproquement: un très fort grossissement n'est utile que s'il est aeeompagné d'un grand pouvoir résolvant. M. E. Abbé a déterminé mathématiquement le rapport qui doit exister entre l'ouverture numérique et le grossissement total (par l'objeetif et par l'oeulaire) du mieroseope (1).

Il a trouvé que ce rapport est lié par une formule assez eompliquée :

$$
1719 \mathrm{~N} \%=o d v
$$

dans laquelle le nombre 1719 est une quantité constante, $\mathbf{N}$, le grossissement total et $o$ l'ouverture numérique de l'objectif; $d$ et $v$ sont deux quantités eonnues, $d$ la distanee de la vision distincte, que l'on éralue ordinairement à 200 millim., et $v$ l'angle visuel (en minutes) sous lequel doivent se présenter les plus petites distanees à observer pour ètre perçues par l'œil. Pour un bon oil, eet angle peut n'ètre que de 1'. Quant à $\lambda$, c'est la longueur d'onde de la lumière employée, et si on la pose $=0,000 \check{3}$, longueur d'onde de la lumière jaunererte, la formule donne une relation, dont tous les termes sont connus, entre $\mathbf{N}$, le grossissement total, et 0 , l'ouverture numérique. De sorte que pour un grossissement donné qu'on veut réaliser, on peut calculer l'ouverture numérique que devra avoir l'objeetif; et

(1) E. AвBE. The relation of aperture and power in the microscope. (Journ. of R. Micr. S., II, 1882). 
réciproquement, pour une ouverture donnée, on peut calculer quel ešt le grossissement que devra fournir le microscope.

Il faut noter que dans cette relation entre le grossissement et l'ouverture numérique, il s'agit dlu grossissement linéaire total, c'est-àdire tel qu'il est produit par l'objectif, la longueur optique du tube et l'action de l'oculaire. Il reste done à itablir, dans ce grossissement total, la part de l'oljectif seul et le rapport qui doit exister, pour un bon fonctionnement de l'appareil, entre le grossissement propre de l'objectif et le «supergrossissement » réaliss par le tube et l'oculaire.

Ce calcul a été fait aussi par M. Abbé (1) et il est trop compliqué pour que nous puissions l'exposer ici, mais en voici les résultats principaux :

Pour une ourerture numérique donnée, le grossissement fourni par l'oculaire et l'allongement du tube peut ètre plus grand, maximum, avec un oljectif à immersion lomogine; il doit ètre plus petit, minimum, avee un objectif à sec. (Il faut encore tenir eompte, dans la détermination de cette valeur, de l'éclairage et de la nature de l'oljet.)

Pour les objectifs à see et à eau, le grossissement par le tube et l'oculaire peut être au maximum de 4 fois en diamètre, environ, et de 6 fois pour les objectifs à immersion homogène.

Pour les objectifs à petite ouverture, eette limite peut ètre portée jusquà 8 et 10 tois en diamètre.

Toutes ces données sont fort utiles, non-seulement aux opticiens, paree qu'elles leur permettent de calculer l'ouverture numérique qu'ils doivent donner à un objectif fournissant tel grossissement déterminé, et réciproquement, - mais encore aux micrographes, qui y trouvent les indieations nécessaires pour tirer le meilleur parti. de leurs instruments et établir des combinaisons satisfaisantes avec les oculaires dont ils disposent et les objectifs dont ils connaissent louverture numẻrique et le grossissement.

A propos du grossissement, nous rappellerons qu'il est plus grand sur l'axe optique, dans le sens de la profondeur, que dans le sens transversal. En effet, le grossissement dans la direetion de l'axe (axial) est direetement proportionnel au earré du grossissement latéral, mais il est inversement proportionnel à l'indiee de réfraction du milieu dans lequel est l'objet.

Ainsi, les objets apparaîtraient extrênément déformés par cette

(1) E. AввÉ. Division of the entire porcer of the microscope between ocular and objective (J. of R. M. S., III, 1883, Zeit. f. wiss. Mikrosk, II, 1885). 
difrérence considérable entre le grossissement en profondeur et le grossissement en largeur, si l'ocil pouvait appréeicr cette différence dans toute son étcndue, et la déformation serait bien plus aceusée si le phénomène se présentait inversement, cest-ì-dire si l'agrandissement etait plus considérable dans te sens transversal. En effet, dans le sens de l'axe on l'apprécie moins, surtout avee les objerstifs a grande ouverture qui ont peu de profondeur. Arce les objectifs a petit angle et a loyer profond, la déformation est très notable et est de nature à tromper tout à fait l'observateur sur la forme réelle de l'oljet, ce qui est encore une cause d'infériorité de ces objectifs.

Quoi qu’il en soit, plus ou moius maryué, le fait est réel.

Nous avons insiste un peu longuement sur ces questions parce qu'elles intéressent autant l'opticien, qui construit les objectifs, que le micrographe qui les emploie, et qu'on les trouve rarement exposées d'une manière intelligible dans les ouvrages français sur le microscope. Nous n'avons pas cru, toutefois, devoir entrer dans des explications plus élémentaires, parce que nous ne faisons pas ici un ouvrage d'optique mierographique, et que nous avons du supposer que nos lecteurs ne sont pas complètement étrangers à la ronnaissance de lit construction et du mode de fonctionnement du microseope.

C'est pourquoi, a propos des objectifs, nous ne nous oceuperons pas des moyens que les opticiens emploient pour corriger ce qu'on appelle l'aberration de sphéricité des lentilles, aberration qui est due à ce que. ces lentilles, en raison de leur forme mème, ne réfractent pas la lunniere igalement dans toutes leurs parties, dans la partie centrale et dans les zones périphériques. Mais hous dirons un mot de l'aberration chromatique, parce que la recherche d'un mode de correction plus complet de cette aberration a amené la construction de nourcaux objectifs, dits apochromatiques.

Tout le monde sait que le prisme dévie, ou réfracte, les rayons de la lumiere: e'est sur ce phénomène qu'est fondéc l'action des lentilles, que l'on peut eonsidérer comme composées d'éléments prismatiques réunis. Nais, cn mème temps qu'il les dèvie de leur direction premicre, il los disperse, c'est-à-dire qu'il sépare le rayon de lumiere blanche en divers rayons coloris dont l'enscmble constitue ce qu'on appelle un spectre.

Le powvoi, réfringent des diverses substances, pouvoir en vertu duquel elles dévient la lumière de sa direction primitive, n'est pas proportionnel a leur pouvoir dispersif, pouvoir en vertu ducuel clles la séparent en un spectre plus ou moins large. C'està-dire qu'une substance peut dévier la lumière autant qu'une autre, 
mais la disperser moins ou davantage. De telle sorte qu'on peut imaginer une combinaison de deur prismes, disposés en sens contraire, telle que les rayons qui la traversent soient redressés dans leur direction primitive, mais restent cncore séparés comme couleurs; ou bien, telle que les rayons colorés soient réunis sous forme de lumière blanche, mais que le rayou blane émergent reste encore dévié de sa direction primitive.

C'est sur cette dernière combinaison qu'est fondé l'achiomatisme, procédé qui permet de faire des lentilles composées qui concentrent la lumière, mais qui ne la dispersent pas en rayons colorés.

Tel est le principe, d'une maniẻre générale. Malhcureusement, les choses ne se passent pas, en réalité, d'une facon aussi simple. C'est qu'en effet le pouvoir dispersif partiel n'est pas proportionnel au pouvoir dispersif général. C'est-à-dire que si une substance donne, dans les mêmes conditions, un spectre deux fois plus long qu'un autre, chacune des diverses parties colorées n'y occupera pas un espace double de celui qu'elle occupe dans l'autre spectre.

Il en résulte que, dans la pratique, il est impossible de superposer complètement, dans toutes leur's parties, deux spectres destinés à s'annuler l'un par l'autre de manière à recomposer la lumière blanche. Il reste toujour's des aberrations chromatiques partielles ou résiduelles qui donnent encore un spectre secondaire, moins sensible, il est vrai, mais qui altère encore la netteté des images. On ne peut supcrposer exactement que deux coulcurs, par exemple dans la partie centrale, de sorte que les objectifs faits avec les lentilles ainsi corrigées donneront encore un champ sensiblement coloré sur les bords, et des images teintées sur leur contour.

De plus, ce spectre secondaire, empèchant toutes les radiations de converger en un foyer unique, donne encore naissance à une aberration chromatique de sphéricité et à une différence chromatique de grossissement pour les différents rayons colorés, résultant de la différence de réfrangibilité de ceux-ci et de leur différence de réfraction dans les diverses parties, centrale et périphériques, de la lentille.

Dans la pratique micrographique, les ubjectifs formés de lentilles ainsi achromatisées ne conservent que des traces peu gênantes des aberrations résiduelles, qu'ils soient sur-corrigés ou sous-corrigés, comme on dit, suivant que ce sont les rayons rouges ou les rayons violets qui dominent dans le spectre sccondaire, l'achromatisation étant faite pour la partie centrale du spectre, la netteté des images est peu altérée.

Toutefois, avec les très forts grossissements et les grandes ouvertures, et surtout quand l'objectif doit scrvir à la micro- 
photographie, les inconvénients de l'achromatisme incomplet deviennent sensibles. Arec tous les verres connus jusqu'a ce jour et la construction la plus parfaite, il est impossible de les éviter et, pour y remédier, il a fallu fabriquer des verres nouveaux.

C'est ce qu'a entrepris, il y a quelques années, lc $D^{r} 0$. Schott, de Witten (Westphalic), qui, de concert avec le prof. E. Abbé ct gràce aux subsides fournis par lc gouvernement prussien, a fabriqué des verres dans lesquels l'acide borique et l'acide phosphorique rcmplacent plus ou moins l'acide silicique et dont la composition est fort complexc.

Dans ces verres, le pouvoir dispersif partiel des divers rayons est à peu près proportionnel au pouvoir dispersif total, et d'après des rapports très divers suivant les échantillons. Plusieurs, ayant le mème indice de réfraction, ont des pouvoirs dispersifs très différents, et réciproquement.

C'est avec ces verres boriques et phosphoriques que M. Zeiss a construit ses objectifs apochromatiques. Ceux-ci conticnnent une lentille de plus que les anciens, et leurs verres sont achromatisés pour trois couleurs, au lieu de deux, de sortc qu'il ne reste qu'extrèmement peu d'aberrations résiduclles ou qu'un spectre tertiaire extrêmement faiblc. L'aplanétisme, e'est-à-dirc la correction de l'aberration de sphéricité, est obtenu pour deux coulcurs du spectr'c.

Voici d'ailleur's, d'après M. Dippel, les avantages des objectifs apochromatiques :

$1^{\circ}$ Utilisation de toute l'ouverture de l'objectif, c'est-à-dire en pratique, possibilité de construire des objectifs de plus grande ouverture utile.

$2^{\circ}$ Reproduction fidèle des couleurs de l'objet.

$3^{\circ}$ Possibilité d'agrandissement considérable de l'image par l'oculaire sans altérer sa netteté, parce que les défauts de l'objectif, qu'un fort grossissement par l'oculaire rend plus sensibles, n'existent plus dans ceux-ci.

$4^{\circ}$ Accouplés avec de nouveaux oculaires, dits compensaterr's, ils donnent une coloration égale de tout le champ et la mème netteté de l'image dans toutes les parties du champ.

Mais le plus grand avantage de ces objectifs est surtout pour la photo-micrographic, parce qu'ils réunissent en un mème point les foycrs chimique et lumineux.

Les objectifs apochromatiques sont donc accompagnés d'oculaires nouveaux, dits compensateurs, qui ont, en effet, pour fonction de compenser les différences chromatiques du grossissement et les aberrations hors de l'axe. Ces oculaires ont la lentille de l'œil plus 
large et la distance de cetté lentille an point oculaire, oi doit s'appliquer l'œil, plus grande. Entin, on peut, eomme nous l'avons dit, réaliser avec cux des grossissements par l'oculaire beancoup plus forts qu'avec les oculaires ordinaires atecouples aux objectifonon apochromatiques. Nous arous v 11 yu'on ne peut gruère, arece ces derniers, sous peine d'altération dans l'image, en employant mème les meilleurs objectifs à immersion lomogèene, grossir plus de 6 fois arec l'oeulaire ordinaire; or, avec les oljectifs apochromatiques on peut employer des oculaires compensateurs grossissint jusqu’a 18 fois.

Il y. a là, comne on le comprend, un avantage tres sérieux, (a) il devient possible, avec un seul objectif apochromatique, de rialiser toute une série de grossissements, limitée sculentent, pour ainsi dire, par l'insuffisance de la lumière. En effet, avec un objectif apocln'omatique grossissant de $\mathbf{1 0}$ diametres et ayant un pouce de foyer, on peut obtenir une série de grossissements par les oculaires contpensateurs $n^{\circ 5} 2,4,8,12,18$, de $20,40,80,128$ et 180 diametres, e'est-à-dire jusqu'à 3.607 fois en surface si l'éclairage est sullisant.

Les objectifs apochromatiques sont fabriques par divers constructeurs, notamment par MII. Zeiss, d'lina; Powell ct Lealand, de Londres ; et Carl Reichert, de Vienne.

\section{Nomenclature des objectifs}

On trouve aujourdhui dans le commerce un grand nombre de bons objectifs. On eomprend que nous ne pourons pas les passer tous en revue et les décrire en détail, nous nous bornerons à signaler brièvement eeux qui nous paraissent devoir rendre les meilleurs services aux diatomistes comme a tous les micrographes qui s'oceupent des recherehes les plus délicates et exigeant les instruments les plus perfeetionnés. C'est d'ailleurs de res objectifs que nous nous sommes servi.

Parmi les objectifs de eonstruction française, nous recommanderons particulièrement ceux de l'ancienne maison Hartnack ot Prazmowski (Bézu, Hausser et Cíe) dont la renommée est depuis longtemps faite.

Ces opticiens construisent trois séries d'objectifs : à sec, à immer. sion dans l'eau, à immersion homogène.

La première série eomprend 9 objectifs à monture fixe, numerotés de 1 i 9 , et ayant des distances foeales, en pouces, de: $2,1,3 / 4$, $1 / 2,1 / 4,1 / \check{\jmath}, 1 / 6,1 / 9,1 / 11$. Ils sont tous à grande ouverture et ont une bonne distance frontale, e'est-i-dire qu'ils laissent un espace suffisant entre la face inférieure plane de la lentille frontale 
et le couvre-objet, pour yu'on puisse employer des lamelles facileinent maniables.

Nous pensons que les diatomistes pourront se contenter des $\mathrm{n}^{\text {os }} \mathbf{2}$ et 4, comme chercheuis, $1^{\circ} \mathrm{g}$, qui est un bon objectif de travail avec $1 / 4$ de pouce de fover, $n^{\circ} 7$ et $n^{0 s} 8$ ou 9.

Le $n^{\circ} 7, \mathbf{1} / \mathbf{6}$ de poute, est un des plus précieux pour les recher-

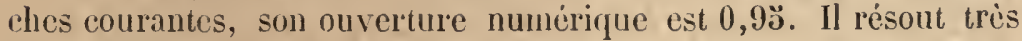
lacilement le Pleurosigma angrolatum dans la lumiere centrale.

Le $1^{\circ} 8,1 / 9 \mathrm{de}$ p., a unc ouverture numérique de 0,97 .

Le $11^{\circ} 9,1 / 11$ de p., a une ouverture numérique de 0,98 .

Tous ces objectifs sont admirablement clairs, donnent un champ trés plan, une inage très brillante et parfaitement définie. Ils supportent sans inconvénient sensible un très fort grossissement par" l'oculaire.

On peut ainsi pousser le grossissement par les oculaires jusqu’à 400 diamitres avec l'objectif $n^{\circ} \mathbf{T}$ et jusqu’à 800 et 1000 diamètres avec les objectif 8 et 9.

Toutefois, nous pensons qu'vu peut remplacer l'objectif $n^{\circ} 9$ a sec par un objectif à immersion.

Lá série des ubjectifs à immersion dans l'cau conıprend 3 numéros: $9,10,13,13$ et 18 , correspondant à des longueurs focales de $1 / 12$, $1 / 16,1 / 2031 / 33$ et $1 / 30$ de pouce.

Tous ces objectifs sont munis d'un systenc de correction pour l'épaisseur du courre-objet, et sont absolument de prenier ordre(1). C'est à leur aide qu'ont été faites presque toutes les découvertes dans le monde des infiniment petits, et e'est de tous ceux qui ont le plus contribué à la connaissance des Diatomées.

Les trois premiers sont les plus pratiques, et éest le $n^{\circ} 9,(1 / 12 \mathrm{de}$ pouce, que nous proposons pour remplacer le $n^{\circ} .9$ at see, quand on posside le $11^{\circ} 8$ et quion est obligé de réduire un pou sa collection d'objectifs.

Le $n^{\circ} 9$ à immersion dans l'eau a une ouverturenumérique de $\mathbf{1 , 2 0}$.

Le $n^{\circ} 10$, le plus célèbre de tous, a 1/16 de p. de foyer et une ouverture de 1,20 .

Ces objectifs résolvent aisément le Surivella gemma, le Frustulia saxonica, l'Amphipleura pellucida, et tous les tests les plus difficiles.

Il en est de mème du n 13 , yui a 1/23̈ de pouce de foyer, et 1,20

(1) Voir pour les détails de la correction et autres :

J. Pelletan. Le Microscope, son emploi et ses applications. Paris, 1876, in $-8^{\circ} 300$ fig.

"Théorie du Microscope d'arrès les idées actuelles. Paris 1888. 
d'ouverture numérique. On peut obtenir avec lui un grossissement utile de 2,0000 à 3,000 diamètres.

La séric des objectifs à immersion homogène de MM. Bézu et Hausser ne comporte que deux numéros, ce qui nous parait absolument suffisant pour les besoins des diatomistes les plus exigeants. Ils portent les $\mathrm{n}^{\text {os }} 9$ et 11 .

Ces deux instruments sont parfaits et certainement valent autant, souvent mieux, que les objectifs similaires du meilleur constructeur allemand (1). Ces opticiens ont remplacé l'huile de cèdre, pour l'immersion, par une solution d'lyydrate de chloral dans la glycérine, solution qui a le même indice 1,52 que cette huile et est d'un usage beaucoup plus commode; on peut l'appliquer sur toutes les préparations sans crainte de les gàter et d'attaquer les vernis; elle est soluble dans l'eau, ne tache pas, et tous les objets qui en ont été mouillés (la lentille frontale des objectifs tout d'abord) peuvent être facilement lavés.

Le $n^{\circ} 9$ à immersion homogène a $1 / 1.2$ de pouce de foyer et une ouverture numérique de 1,25 à 1,$30 ;$ le $^{\circ} 11$ a $1 / 18$ de pouce de foyer et une ouverture de 1,23 .

Les objectifs de MM. Bézu, Hausser', sont accompagnés d'une belle séric d'oculaires dont l'un, le $\mathbf{n}^{0} \mathbf{2}$, est 'muni d'un micromètre portant un eentimètre divisé en 10 parties et que l'on peut mettre au point à l'aide d'un collier.

Nous avons signalé M. C. Reichert de Vienne (Autriche), pour la bonne construction de ses microseopes, nous pouvons le citer aussi pour ses objectifs dont la séric est fort nombreuse.

Les objectifs à sec, dont plusieurs sont à correction, sont au nombre de 14 , depuis 2 p. $1 / 2$ de foyer jusqu'à $1 / 12$, avec des ouvertures numériques allant de 0,17 à 0,98 .

Les objectifs à immersion dans l'cau, avec eorrection, sont au nombre de $\check{5}$, avec des foyers de $1 / 15 \%, 1 / 18,1 / 20,1 / 30$ de pouce et des ouvertures numériques variant de 1,10 à 1,20 .

Trois objectifs à immersion homogène, de $1 / 15,1 / 20,1 / 30$ de pouce de foyer, ont des ouvertures numériques variant de 1,23 à 1,40 .

M. C. Reichert construit aussi maintenant des objectifs apochromatiques, accompagnés d'oculaires compensateurs qui sont d'excellente qualité.

Nous ne nous attarderons pas à la description des objectifs de construction étrangère qui sont fort nombreux, mais nous pensons que tout ee qu'on va chereher à l'étranger peut se trouver dans notre pays.

(1) Voir Journal de Micrographie, T. IX, 1885, p. 313. 
L'opticien qui a construit les meilleurs objectifs du monde fut R. B. Tolles, de Boston, mort malheureusement, il y a quelques années. Nous avons été jadis le zélé propagateur, en France et sur le continent, de ces admirables instruments. Aujourd'hui, le fils de son ancien maitre Ch. Speneer, de Geneva, est à notre avis le premier opticien du Nouveau-Monde.

En Angteterre, MM. Powell 'et Lealand oceupent le premier rang et leurs objectifs sont tout à fait supéricurs. Nous pouvons eiter surtout le $1 / 8$ de p. (new formula), à immersion dans l'eau, qui est célèbre, le 1/16 à cau et le $1 / 12$ à immersion homogène, etc.

Malheureusement, les objectifs anglais et amérieains sont d'un prix tellement élevés qu ills restent à peu près inabordables pour la majorité des micrographes.

En Allenagne, les constructeurs ne font pas très cher, mais ils font grossier, et nous navons à eiter que M. C. Zeiss, bien connu d'ailleurs, et qui a construit les premiers objectifs à immersion homogène. Ce constructeur, aidé pour la partie théorique par son beau-frère, le professeur Abbé, oceupe aujourd'hui une des premières places dans l'optique scientifique. Il a certainement réalisé un progrès considérable pour l'Allemagne où, jusqu'à lui, on eonstruisait fort mal. Toutefois, nous, avons dit que, pour la partic méeanique, les bons instruments francais, tels que ceux que nous avons déerits, sont très supérieurs aux meilleurs instruments allemands, et pour la partic optique, on trouve aussi bien en France.

Nous devons, cependant, ajouter quelques mots relativement aux objectits apochromatiques que construit II. C. Zeiss.

Ces objectif's composent trois séries : une série à sec, une série à inmersion daus l'eau, une séric à immersion homogène.

La série à sec comprend trois numéros: un objectif de $2 / 3$ de pouce (16 millinı.) de foyer, avec une ouverture numérique de 0,30 ; un objectif de $1 / 3$ de pouce ( 8 millim.) de foyer, avee une ouverture de 0,60 ; un objectif de $1 / 6$ de p. ( $\$$ millim.), avec une ouverture de 0,93 . - Tous sont construits pour fonctionner avee, une longueur de tube de 16 eentimètres (tube des mieroseopes continentaux) et 18 centimetres en comptant la hauteur de la monture (1).

Il n'y a, actuellement, qu'un seul objectif à immersion dans l'eau (avec correction). Il a 1/10 de p. de foyer et une ouverture numćrique de $1,23$.

La série des objectifs apochronatiques à immersion homogène

(1) Une autre série est construite pour les tubes anglais, de 25 centimètres Elle comprend 3 objectifs de 1 p., $1 / 2$ p. $1 / 4$ de p. $(24,12,6$ millimètres) avec des ouvertures numériques $0,30,0,60,0,95$. 
comprend deus numéros doubles : deux objectifs de $1 / 8$ de $p$. (3 millim.) dont l'un a une ouverture numérique de 1,30 ct l'autre de 1,10; deux objectifs de $1 / 12$ de $p$. avec ouverture de 1,30 et de 1,40. - Les uns et les autres sunt sans systeme de correction.

Il y a aussi 6 oculaires compensateurs, dont les grossisements sont respectivement $1,2,4,8,12,18$.

\section{\$3. - CONDENSATEURS ET APPARELS DIVERS}

La résolution des liatumés ef particulicrement des espèces difficiles, telles que le Frustulia saxonica, le Surirella gemma. l'Amphipleura pellucida, cte., cxigent non seulement l'emploi des meilleur's objectifs, mais divers artifices de lumicre que l'on réalisait autrefois a l'aide d'un grand nombre d'instruments divers, condensateurs, réllecteurs, illuminateurs, éclairages ubliques, paraboloïdes, etc. - dont le perfectionnennent des ubjectifs à immersion homogene a de plus en plus restreint l'emploi et qui sont, a peu près tous, remplacés aujourd'hui par l'apparcil qu'on appelle éclairage ou condensateur d'Abbé.

Il y a, d'ailleurs, beancoup de rondensateur's, et l'idée de remédier a l'absorption considérable de lunicre que produisent les objectifs puissants en concentrant sur l'objet un large còne de lumière a depuis longtcmps été appliquée par Wollaston, Amici, Dujardin, ete.

Il faut avoucr cependantque, sauf un tres petit nombre, les micrographes français ignoraient encore à peu press, il I a quelques années, l'emploi des condensateurs. On savait bien qu il existait chez les opticiens des instruments destinés à augmenter l'ërlairage. des objets, mais ils étaient en général considérés, par la plupart des microscopistes, un peu comme ces closes théoriques yui sont inscrites dans les livres et dont parlent les gens yui se piquent d’érudition, mais que personne na jamais vues. En Ingleterre et en Amérique, au contraire, tous les merographes se servaient depuis longtemps d'un grand nombre de ces condensateurs ou condcnseurs; il ne se passait jas de jour que les Revues spéciales né donnissent la description d'un nouvol appareil d'éclairage, et les opticiens construisaient et vendaient couramment des instruments, iquelquefois tris complipués et très coùteux, comme les condenseurs de Beck, de Ross, de Powell et Lealand, Tolles, etc.

Nous avons été le premier en France à donner une description 
détaillée de ces instruments (1), à en adopter l'emploi et à en cxpliquer lc maniement, et c'est certainement gràce à notre propagande que les micrographes de notre pays ont compris les nouvelles ressources que res divers appareils mettaient à leur disposition. C'est ainsi que nous avons introduit en France, déerit el employé le premier l'óclairayer d'Abbé, pour ainsi dire dis son apparition (2), lequel a ité depuis complitement adopté par nos micrograplies, imité par nos opticiens, romme invention allemande, et n'est cependant, pour le fond: qu une vicille invention française.

Nous signalerons rapidement quelyues uns de res appareils dont plusieurs sont aujonrd'lui un peu abandonnes pour l'éclairage d'Ablı́, mais dont rertains rendent encore d'excellents services.

Condensateur de Dujardin. - C'est ce que les opticiens appellent un enndensateur direct. L'expression est mauraiso: c'est un condensateur cential ou plutòt axial, e'est-à-dire qu'il concentre sur l'oljet un faisceau de lumirire venant dans la direction de l'axe optique et non dans une direction oblique.

Cet instrument, composé de trois lentilles achromatiques comprises dans une monture que l'on établit sous la platine, réalise un oljjectif à court foyer yue l'on place de manière à ce que l'objet soit à son foyer. Il a été l'origine de tous les condensateurs qui ont été construits depuis et leur a servi de modèle.

Il a d'ailleurs été considérablement perfectionné et son ouverture extrèmenent agrandie, cn même temps que g̣randissait l'ouverture des objectifs. Aujourd'hui, MI. Bézu, Hausser et Cie construisent un condensateur direct qui n'est autre que celui de Dujardin perfectionne et mis au niveau du progrès actuel, condensateur qui remplace eomplètement l'appareil d'Abbé fonetionnant avec la lumière centrale, éest-ì-dire dans I axe. Nous ajouterons mème qu'cn le comparant aver les meilleurs condensateurs des fabriques allemandes, nous avons trouvé celui de MM. Bézu très notahlement supéricur. Aussi, nous n’hésitons pas à donner la préférence à cet instrument toutes les fois qu'il s'agit d'obtenir une concentration de lumière dans l'axe, sans faire intervenir l'éclairngc oblique. Du reste, cet éclairage oblique, чui est absolument défectueux, qui déforme les oljets, donne des images colorées, dénaturées, illusoires, disparait de plus en plus des pratiques micrographiques, à mesure que les objectifs et les instruments d'éclairage sc perfec-

(1) J. Peli.etan: Le Microscope, son emploi et ses applications. $1 \mathrm{v}$. in. $8^{\circ}$ 1876 " Etudes sur les microscopes étrangers. $1 \mathrm{v}$. in $-8^{\circ} 1878$. \Journal de Micrographie, 1877-1888 (passim).

(2). J. Pelletan : Le Microscope, son emploi, etc., 1876 p. 116. 
tionnent. On arrive, en effet, à avoir des images exactes des objets, avec leur's détails les plus délicats, en agrandissant l'ouverture numérique des objectifs, ce qui permet d'admettre pour la formation de l'image un plus grand nombre des pinceaux diffractés produits par le pinceau central ou axial, sans faire intervenir les images complexes et inexactes qu'amènent les rayons obliques employés seuls, comme dans l'éclairage dit oblique.

Au licu de réfléchir la lumière à l'aide du miroir sous le condensateur, Dujardin se servait d'un prisme placé horizontalement afin d'obtenir, sur une des faces intérieures du prisne, le phénomène de la réflexion totale, réflexion qui est plus complète que sur le miroir étamé et n'est pas double. Cette disposition est fort bonne et trìs souvent employée, surtout par les micingraphes étrangers qui se servent encore beaucoup plus que mous du condensateur.

L'objet, quand on emploie le condensateur, doit ètre placé sensiblement au fover de ec depruier, aussi est-il préférable, aree la lumière du jour, de se servir pour éelairer l'appareil, du miroir plan ou du prisme à réflexion totale qui réfléchissent parallèlement les rayons solaires parallèles, déterminant un foyer fixe, le foyer principal du système. Avec le miroir concave, on aurait un foyer conjugué du foyer du miroir, foyer yu’il faudrait clicrcher en reculant le condensateur de l'objet.

Avec la lumière des lampes, on peut rendre les rayons parallèles cn leur faisant traverser une grosse lentille d'éclairage au foyer principal de laquelle on place la flamme éclairante de la lampe. Les rayons émergeants sont alors parallèles et l'on peut opérer avee le condensateur comme s’il était éclairé par la lumière du jour.

MM. Bézu et Hausser construisent le condensateur de Dujardin avec un foyer de 2 millimètres, de sorte qu'en l'élevant sous la platine jusqu'au contact de la face inféricure du porte objet, et en employant un porte objet de 2 millimètres d'épaisseur', l'objet déposé au point optique, sur la face supérieure de celui-ci, se trouve au foyer du condensateur. On peut alors mettre une goutte d'eau ou de glycérine entre le condensateur et le porte oljet, pour établir une continuité optique entre les deux verres et éviter la perte de lumière par les réflexions sur les deux surfaces.

C'est sur un principe semblable que sont construits le condensateur à immersion de Powell et Lealand et le traverse-lens de Tolles.

L'appareil est souvent réduit, comme dans les derniers instruments que nous venons de citer, à une seule lentille (Fig. 83), presqu'une demi-boule que l'épaisseur du porte-objet vicnt compléter avec l'intermédinire d'une goutte d'cau, de glycérine ou 
d'essenee de eìdre. C'est ce qu'on appelle des condensrteurs à immersion (1).

On peut transformer le eondensateur de Dujardin de manière à lui faire donner l'éclairage oblique, en adaptant sous la lentille inférieure une coulisse dans laquelle on pousse une lame de métal pereé d'un trou plus ou moins petit. Si le trou est amené au eentre de la lentille, l'instrument donne l'éelairage axial à l'aide d'un pineeau plus on moins réduit suivant le diamètre du diapluragme. Mais si le trou est maintenu lor's de l'axe, l'instrument ne reçoit plus qu'un pineeau oblique et d'autant plus oblique que le trou est plus exeentrique.

M. de Castracane a imaginé de pratiquer une fente latérale dans la monture du eondensateur au dessous de la première lentille, la frontale; e'est dans eette fente qu'il introduit une lame de métal très minee et percée d'un trou. Le diaphragme est ainsi placé entre la première et la seeonde lentille au lieu d'ètre au dessous de la troisième. Cette disposition est préférable.

Condensateur achromatique de Beck. - Cet instrument est la réalisation anglaise de l'idée de Dujardin. Il eonstitue un objectif à grand angle d'ouverture, eomme on disait alor's, $10 \ddot{3}^{\circ}$, et même $160^{\circ}$. Le foyer est eourt; on abaisse ou on élève l'instrument pour porter le foyer sur l'objet, et l'on éclaire avec le miroir plan ou le prisme à réflexion totale.

On obtient l'éelairage oblique avee un diaplıragme placé au dessous de l'instrument et constitué par un disque tournant pereé de trous de différents diamètres et d'ouvertures ne laissant passer' que les rayons marginaux ou les pinceaux venant d'une partie sculement de la zone marginale.

Condensateur achromatique de Ross. - Ce condensateur ne représente qu'un objeetif de $2 / \breve{3}$ de pouce de foyer avec un angle d'ouverture de $110^{\circ}$. On le règle et l'éelaire eomme le préeédent. Il donne la lumière oblique et marginale à l'aide de diaphragmes tournants placés aussi sous la troisième lentille. Il y a deux disques placés l'un au dessus de l'autre. Le premier porte 8 trous qui font décroittre l'angle au sommet du pinecau éelairant de $110^{\circ}$ à $20^{\circ}$. Le sceond porte 3 trous obturés au eentre et plusicurs trous pour la lumière latérale. La tranche des disques porte des chiffres qui indiquent la valeur de l'angle du eòne lumineux réduit par chaque

(1) Voir J. Mayart. - Les éclairages d immersion. (Jour. de Micrographie, T. III, 1879, p. 178:) 
ron, et des lettres qui font connaitre la nature de chaque écran, à touverture marginale ou latérale, qu'on a amenc dans l'ave.

Condensateur achromatique de Powell et Lealand. - C'est un instrument analogue, mais realisant un olyjectif (le beaucoup) plus grande ouverture $\left(170^{\circ}\right)$.

Nous avons dit que MM. Powell et Lealand construisent aussi un conclensateur à immersion simplifié, très bon instrument dont les: détails que nous avons domnés ì propos des rondensateurs de Dujardin nous dispensent de reproduire la deseription.

Nous ne disons rien des condensateurs de Webster et de loaucoup d'autres qui rentient aussi dans le principe de eelui de Dujardin.

Paraboloïde de Wenham. - Cet appareil consiste en un evlindre de verre dont la base est coupée perpendiculairement à l'axe, et dont la partie supérieure est taillée en un paraboloïle de révolution tronqué à son sommet de manière que le foyer du parabolö̈de tombe en dehors de la troncature et, par exemple, a 2 ou 3 millimètres au delà. De sorte que quand l'instrument est placé verticalement sous la platine, on peut le disposer de manière à ce que le fover du parabolö̈le coincide avec le point optique. L'oljet qu'on aura déposé en ee point sera done éclairé virement par tous les rayons qui, envoyés par le miroir plan du microscope (rayons parallèles), frapperont la base du ç̣lindre normalement et pénétreront par conséquent sans déviation dans la masse de rerre. Ils viendront alors se réfléchir totalement sur la face interne du paraboloïde et seront tous réfléchis vers le foyer.

Mais si le sommet du parabolö̈de était simplement tronqué par un plan, les rayons y subiraient des réflevions sur la face interne et des réfractions a la sortie qui les empicheraicnt de converger au foyer. Aussi, la troncature a-t-clle la forme d'une surface sphérique concave ayant pour centre le foyer du parabolö̈de. Tous les rayons, après s'ètre réfléchis totalement sur la surface parabolique interne, viennent frapper la surface sphérique de la troncature normalement à cette surface, la traversent sans déviation et convergent au centre de la sphère qui est en mème temps le foyer du parabolö̈de. L'objet se trouve ainsi vivement éclairé.

Cet instrument est done un condensateur par réflexion, et non plus par réfraction comme les précélents. Il y a seulement une réfraction accidentelle produite par l'épaisscur de la lame porteobjet, réfraction qui a pour effet d'abaisser un peu le point de 
convergence des rayons. On peut, du reste, rendre cet effet très peu sensible en employant un portc-objet très mince.

Le paraboloïde de Wenham n'a pas été construit tout à fait dans le but de servir de condensateur, mais pour produire un éclairage de l'objet sur un fond noir. En effet, le fond de la cupule sphérique qui forme la troncature est obturé par un disque en métal noirci dont on peut régler la hauteur et qui arrète tous les rayons venant directement de la base du cylindre, ne laissant passer que les rayons latéraux réfléchis sur la surface parabolique intérieure.

Cet instrument est fort commode; il permet un éclairage très

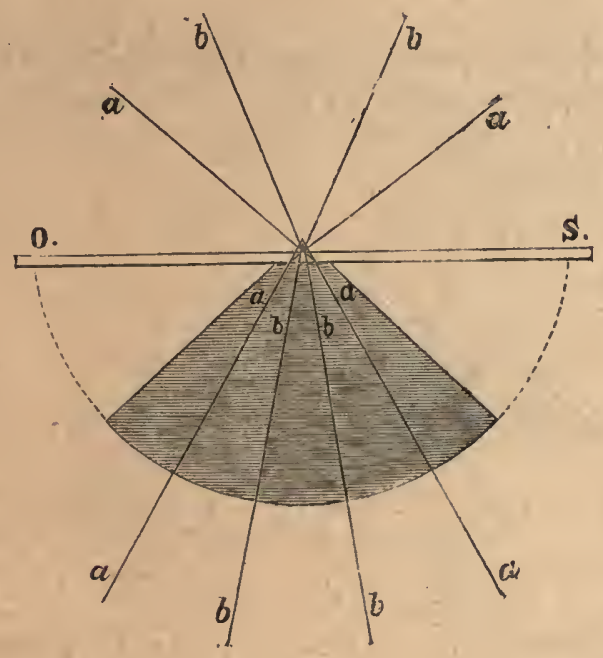

Fig. 83. - Condensateur hẻmisphérique de E. Gundlach. Schéma de la marche des rayons.

oblique, presque rasant, tout autour de l'objet. On obtient ainsi une véritable illumination des objets, sur un champ noir, qui donne des images très saisissantes.

Employé arec le microscope binoculaire, il produit des effets magiques. Malheureusement, comme on le comprend, le paraboloïde de Wenham, ainsi employé, n'est possible qu'avec des objectifs de faible grossissement et de peu d'ouverture.

Il peut rendre toutefois, comme condensateur simple, de véritables' services.

M. Nachet a réalisé, avec un cône de verre tronqué au sommet, un éclairage sur fond noir qui, moins parfait au point de vue 
théorique que le paraboloïde, donne cependant des effets assez satisfaisants.

Reflexe-illuminateur de Wenham. (Reflex illuminator). Ce petit appareil, peu commode, n'est plus guère employé depuis le perfectionnement des condensateur's ordinaires. Il consiste en une pièce de verre recevant par-dessous les rayons émanés du miroir normalement à sa base et, par une troncature oblique, les renvoyant, après une réflexion totale sur la face intérieure de la troncature, suivant une incidence presque rasante sur l'objet placé au point optique.

Le Prisme de Woodward, le Projecteur oblique de Gundlach, et autres instruments destinés à réaliser un éclairage très oblique, pour faciliter la résolution des tests difficiles, sont aussi très délaissés maintenant (Fig. 84).

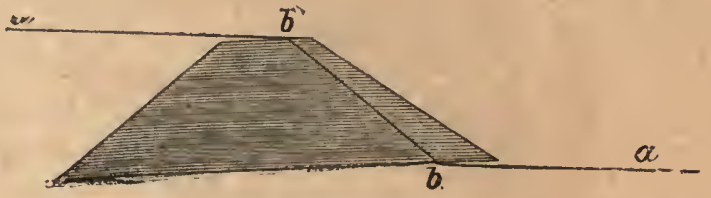

Fig. 84. - Projecteur de Gundlach (marche du rayon $a b$ ).

Vertical-illuminateur de Beck. (Vertical illuminator). Cet instrument se compose d'une douille qui se visse sur le microscope au-dessus de l'objectif. Elle porte par en bas un pas de vis sur lequel on adapte l'objectif. La hauteur du tube est ainsi augmentée de la hauteur de la douille. Celle-ci est percée, sur le còté, d'un trou devant lequel, dans l'axe du tube, est placée une petite rondelle de verre très mince (un couvre-objet) incliné à $40^{\circ}$ sur l'axe. Si l'on place une lumière devant le trou latéral, les rayons qui pénétreront par cette ouverture iront frapper la lamelle de glace, se réfléchiront par en bas, suivant l'axe, traverseront l'objectif, qui servira ainsi de condensateur, et iront se concentrer au foyer de cet objectif, c'est-à-dire sur l'objet.

La lamelle de glace n'occupe pas tout le diamètre du tube, mais seulement la partie centrale, de sorte qu'elle ne gène pas sensiblement la marche des rayons qui, partis de l'objectif, vont former l'image dans l'oculaire. De plus, elle est très mince et parfaitement ransparente. 
Condensateur achromatique de Swift. - Cet appareil est plus qu'un condensateur ordinaire; il peut remplacer à lui tout seul la sous-platine des instruments anglais.

Il se compose d'abord d'un condensateur proprement dit A (Fig. 83), c'est-à-dire d'un système optique de $2 / 3$ de pouce de foyer et de 0,93 d'ouverture numérique ( $140^{\circ}$ ouverture angulaire).

Sous les lentilles de ce système est un diaphragme à contraction que l'on manœuvre à l'aide du petit levier B. Le disque tournant E est percé de quatre cellules rondes, dans lesquelles on peut placer des rondelles diaphragmes à ouverture plus ou moins large ou à

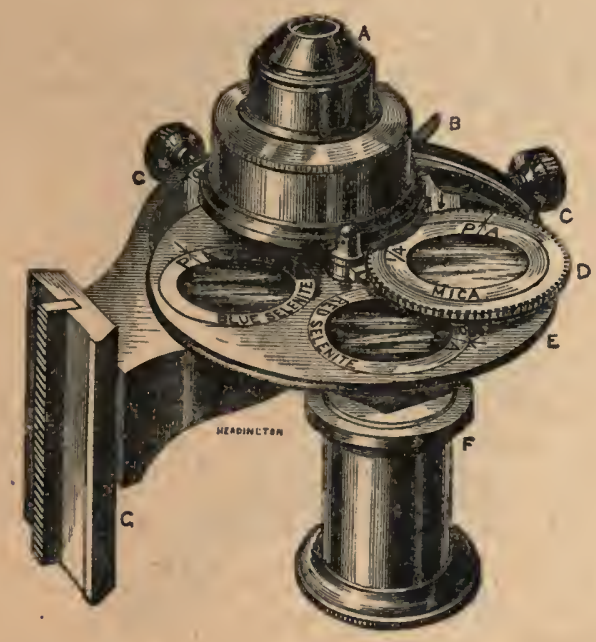

Fig. 85. Condensateur achromatique avec polarisateur, de J. Swift.

éclairage latéral, ou des lames de sélénite pour la lumière polarisée. On peut amener toutes les cellules dans l'axe du condensateur et les rondelles y peuvent tourner autour de leur centre. Enfin, deux cellules opposées diamétralement $D$ (une seule est visible dans la figurc) peuvent ètre amenées dans l'axe au-dessus du plan du disque $\mathbf{E}$, et recevoir des rondelles de mica tournant autour de leur centre, lorsqu'on opère avec la lumièrc polarisée. Un prisme polariseur $\mathrm{F}$, porté par un excentrique, peut êtrc poussé dans l'axe optique.

Cet instrument se monte sous le microscope à la place de la sousplatine, à l'aide de la crémaillère $G$ et se centre avec les petites vis $\mathrm{C}, \mathrm{C}$. 
Condensateur ou éclairage d'Abbé. - Nous arons déjà dit que le condensateur proprement dit qui fait partie de cet instrument n'est pas nouveau; on le construisait depuis longtemps en France, lorsque M. Abbé eut l'idée d'y faire adapter par II. Zeiss un systè me spécial pour l'agencement et la manipulation des diaphragmes. Ce système, extrèmement commode, bien que le mécanisme en soit le plus souvent assez grossièrement construit en Allemagne, a fait de l'Eclairage d'Abbé le complément à peu près indispensable de tous les microscopes destinés aux études les plus délicates.

Nous avons été le premier à en apprécier la valeur, à l'introduire

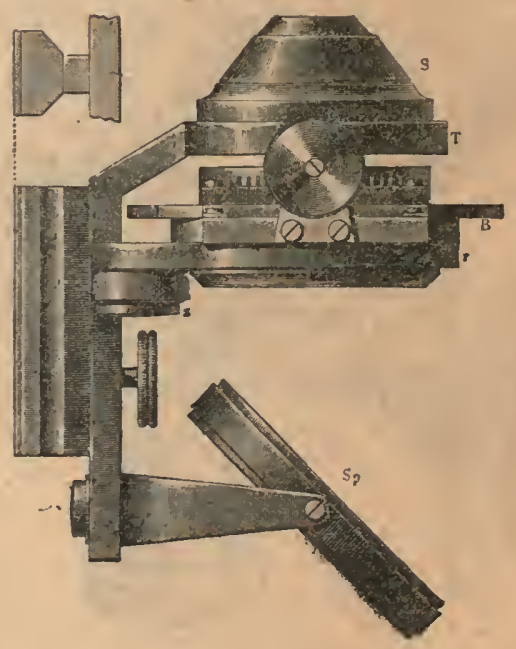

Fig. 86. - Condensateur d'Abbé.

en France, à le décrire (1) et à le recommander aux micrographes. Depuis lor's, cet instrument a été adopté partout, et a remplacé à peu près tous les autres appareils modilicateurs de l'éclairage. Il se. compose de deux parties. (Fig. 86.)

La première est le condensateur proprement dit, S, composé de 2 ou 3 lentilles, suivant le modèle, et constituant un système optique

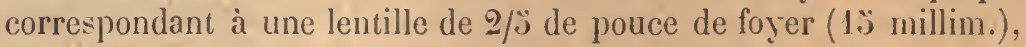
mais n'ayant que 2 millimètres de distance frontale réelle. Ce système doit ètre monté sous la platine de manic̀re que la face supérieure de la lentille frontale aflleure le niveau supérieur de cette platine et

(1) J. Pelletax: Le Microscope, son emploi, ete. p. 115. 
vienne au contact du porte-objet, lequel, ayant un peu moins de 2 millimètres d'épaisscur, aura le foyer du ș̣stème sensiblement au niveau de sa face supćrieure. On peut établir une immersion entre la lentille et le porte-objet pour constituer un milieu optique homogène.

Ce condensateur est porté par un anneau T, soutenu par un bras horizontal tenant à une pièce métallique qui se fixe sous la platine et porte, en has, un miroir Sp, plan et concave.

La seconde partie de l'instrument est portée par l'anneau $r$. Celui-ci est soutenu par la mêmepièce métallique, à l'aide d'un excentrique qui tourne autour d'un pirot $z$ et pęrmet d'amener tout ce système en dehors de l'axe optique à droite de la platine.

L'anneau $r^{2}$ se trouve ainsi découvert, ainsi que le plateau B qu'il supporte. Celui-ci est percé d'une large ouverture ou cellule dans laquelle on peut placer des rondelles diaphragmes percées de trous de différents diamètres ou des écrans à fentes marginales ou latérales. La cellule étant ainsi garnie du diaphragme choisi, on repousse le système sous le condensateur, dans l'axe optique.

Le plateau B porte une crémaillère sur laquelle roule un pignon commandé par le bouton moletté $g$. Si l'on tourne le bouton dans un sens ou dans l'autre, on fait avancer ou reculer le plateau $B$ et le diaphragme qu'il porte sur la monture $r$ fixe. Le pinceau qui passe par le trou du diaphragme devient ainsi de plus en plus oblique en avant ou en arrière. Mais, de plus, en poussant le bouton $g$, sans le faire rouler sur la crémaillère, on peut faire tourner le plateau B sur l'anneau $r$, par un mouvement de rotation autour de l'axe optique. On peut ainsi éclairer obliquement la préparation de tous les côtés, et diriger le pinceau lumineux dans tel sens que l'on veut, par exemple perpendiculairement à certaines stries de l'objet pour en faciliter la " résolution ».

Cet instrument, dont le maniement est facile et dont on apprend très vite à se servir, est extrèmement commode, et l'on résout aisément à son aide les tests les plus difficiles. On le construit maintenant partout, et bien qu'il aitété un peu modifié dans sa forme par les divers fabricants pour l'adapter à leurs instruments, le principe est resté le méme: un système condensateur, analogue à celui de Dujardin, pourant arriver au contact de la préparation; et, par-dessous, un mécanisme permettant de mouvoir des diaphragmes dans un plan perpendiculaire à l'axe optique et de les faire tourner sur eux-mèmes, dans - ce plan, autour de l'axe optique comme centre.

C'est la réalisation de ce mécanisme qui constitue l'invention de M. Abbé. 
Chambres claires. - Il ne rentre pas dans notre plan de décrire un plus grand nombre d'appareils accessoires, qui, du reste, n'intéressent pas d'une manière particulière les diatomistes. Nous signalerons, seulement pour mémoire, les chambres claires qui sont nécessaires pour dessiner ad naturam les objets microscopiques et pour en évaluer le grossissement.

Il y a un très grand nombre de ces instruments et beaucoup de bons modèles, mais tous exigent de l'opérateur une certaine habitude pour pouvoir s'en servir utilement. Nous rappellerons spécialement

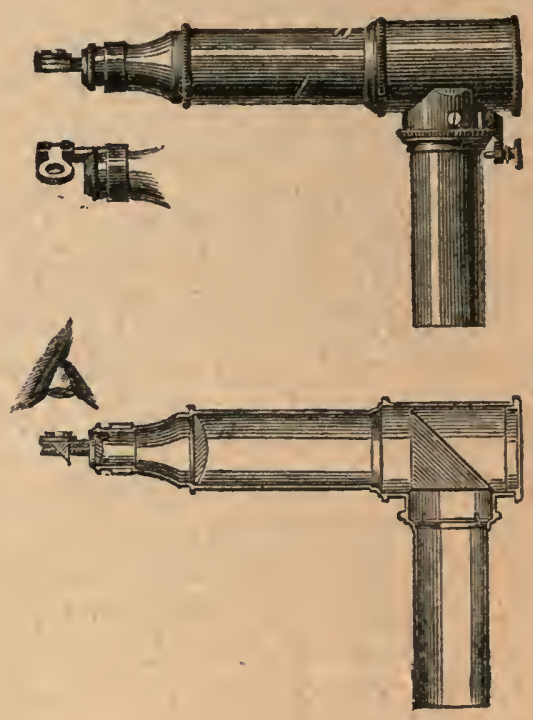

Fig. 87. - Chambre claire d'Oberhaeuser (construite par MM. Bézu, Hausser et $\mathrm{Cie}^{\mathrm{ie}}$.

les modèles de M. Govi, de M. Malassez, de M. Hofmann, et celu i d'Oberhaeuser qui, bien qu'ancicn, n'en est pas moins un des meilleurs et des plus commodes. Cettc chambre claire s'applique à tous les microscopes disposés verticalement; elle sc met à la place de l'oculaire, qu'on enlève, et transporte le champ sur la table au pied du microscope. (Fig. 87.)

Nous n'avons pas à donner ici la théorie de ces instruments qui permettent de voir à la fois le champ du microscope et une feuille de papier placée sur la table et paraissent, par conséquent, transporter l'image sur le papier, où l'on pcut la dessiner. Nous 
renverrons pour toutes ces questions, ainsi que pour la mesure des grossissements, aux ouvrages de mierographie générale (1).

\section{§ IV. - ÉCLAIRAGE}

Un se sert pour éclairer le microscope de toutes les sources de lumière, et nous n’avons pas à apprendre iei à nos leeteurs eomment on en dirige les rayons sur l'oljet. Yous ferons seulement remarquer que pour la résolution des tests diffieiles et particulièrement des Diatomées délicates, eomme les Surirella gemma, Amphipleura pellucida, ete., il est besoin d'une lumière très intense.

La lumière émanée d'un nuage blane fortement éclairé par le soleil est une des plus propiees, mais la lumière solaire direete aide extraordinairement à la résolution des plus fines stries des Diatomées, résolution qui devient alors très faeile, mème avec des objectifs de qualité secondaire et d'ouverture numérique modérée, pourvu que le grossissement soit assez fort pour « séparer " nettement ces stries.

Lumière solaire monochromatique. - Seulement, il est indispensable que cette lumière solaire qui, si on la recevait telle quelle dans l'œil à travers les systèmes optiques, ne ferait qu'aveugler l'observateur, il est indispensable, disons-nous, que eette lumière soit monochromatisée, e'est-à-dire réduite à une seule des radiations colorées qui composent la lumière blanehe, et c'est la lumière bleue qui agit le mieux en fatiguant le moins.

Pour réaliser eet éelairage monochromatique, il y a un moyen très simple qui consiste à faire traverser aux rayons solaires, avant de les diriger sur le miroir, une cuve en verre à faces parallèles de quelques eentimètres d'épaisseur et eontenant une dissolution d'un sel de euivre ammoniacal, sulfate ou azotate, parfaitement limpide, du reste:

On obtient, comme on sait, cette solution en dissolvant dans de l'eau distillée une certaine quantité de cristaux de sulfate ou d'azotate de euivre. On ajoute alors de l'ammoniaque, qui produit aussitôt un préeipité blanc bleuàtre d'hydrate d'oxyde de cuivre. On verse de l'ammoniaque avec précaution, en agitant la liqueur avee une baguette de verre, et le précipité ne tarde pas à se dissoudre. Il ne

(1) Voir J. Pelletan. - Le Microscope, son emploi et ses applications. 1 vol. 775 p. et 300 grav., in-8. Paris, 1876. 
reste plus qu’à filtrer et à étendre, au besoin, la solution avec de l'eau distillée si sa nuance est trop foncée.

La cuve de glaces à faces parallèles est alors placée derant le miroir de manière à ce que les rayons solaires traversent la couche liquide avant d'arriver au miroir. On est frappé alor's de la facilité avec laquelle toutes les Diatomées se résolvent dans la lumière oblique et même avec un éclairage axial.

On a cherché à remplacer la solution de sel de cuivre par un écran formé avec une lame de verre bleu au cuivre. Les résultats sont beaucoup moins bons, néanmoins cette disposition peut souvent ètre utile. On a placé aussi des verres teintés en bleu plus ou moins

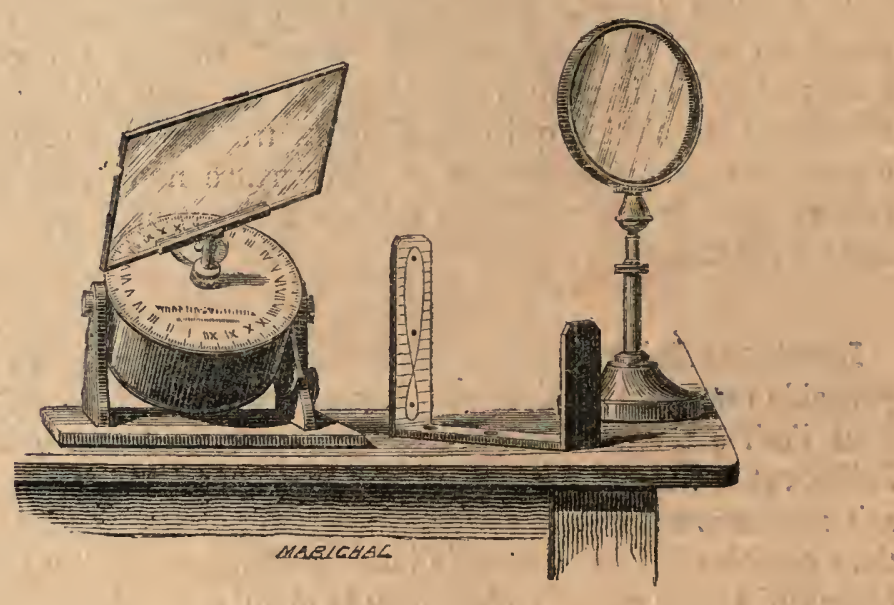

Fig. 88. - Héliostat de Prazmowski.

foncé sous la platine, sous la préparation ; puis, sur la lentille de l'œil (de l'oculaire), et cela facilite souvent la perception des fines stries. II. E. Mauler, de Genève, a même réussi à préparer des Diatomées sur une lame porte-objet en verre bleu avec un couvre-objet pareillement en verre bleu, et la résolution de ces tests s'est trourée notablement facilitée.

On se servait davantage de la lumière monochromatique autrefois, avant qu'on possédât les objectifs puissants et perfectionnés que l'on a aujourd'hui. Néanmoins, nous recommandons ce procédé commode et facile de résolution des Diatomées. On peut compléter cette installation avec l'excellent héliostat de Prazmowski (Fig. 88), petit instrument fort ingénieux, le moins coùteux de tous les hélios- 
tats, et qui, immobilisant les rayons solaires dans une même direction donnée, permet de résoudre immédiatement tous les tests et toutes les Diatonıées imaginables sans aroir à déplacer continuellement le microscope pour suivre la marche du soleil.

Ajoutons que nous ne saurions trop recommander l'hćliostat de Prazmowski aux cxpérimentateurs qui font de la micro-plotograplie et à qui cet intrument est indispensable (1).

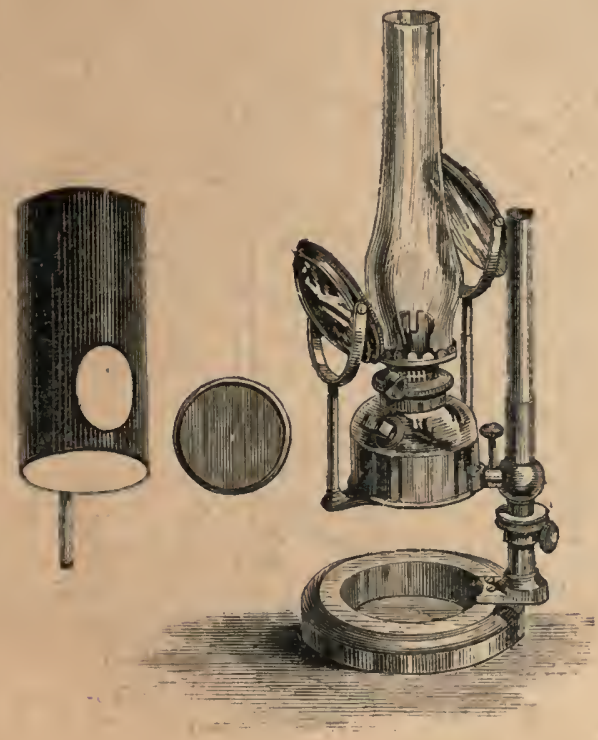

Fig. 89. - Lampe de Swift.

Lampes. - La lumière des lampes à huile, à gaz et à pétrole est aussi fort employée pour le microscope, et surtout celle des lampes à flamme plate. Il est utile de rendre les rayons parallèles, cn plaçant la flamme au foyer principal d'une lentille convergente, avant de la recevoir sur le miroir du microscope.

On a construit beaucoup de modèles de lampes à écrans opaques, à réflecteur, à lentille, etc., pour le microscope. Nous représentons ci-dessus la lampe dite de Swift, qui est bien comprisc, montée sur un pied extensible, munie d'écrans et de tous les accessoires utiles, et

(1) Voir Journal de Micrographie, 187\%. T. I, p. 130. 
qui porte une tubulure latérale pour le remplissage. M. Deyrolle fabrique maintenant une lampe analogue.

Éclairage électrique du microscope. - Depuis quelques années, on emploie les lampes électriques à incandescence pour éclairer le microscope. C'est à M. H. van Heurck, que l'on doit les premiers travaux dans ce sens, travaux qui datent de l'Exposition Universelle de Paris en 1878, mais qui ne sont guère arrivés qu'en 1881 à des résultats pratiques (1).

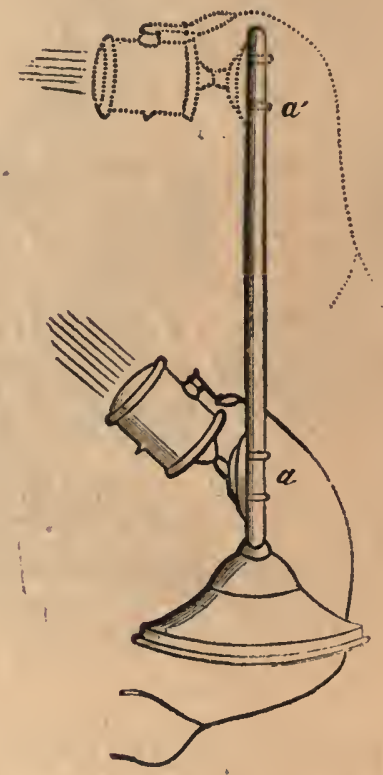

Fig. 90. - Photophore Hélot-Trouvé et Van Heurck, pour l'éclairage électrique du microscope.

Ce sont des lampes Swan modifiées par M. Stearn qui ont d'abord servi à M. Van Heurck. Ces lampes étaient de fort petite dimension de telle sorte que l'arc lumineux, en raison de la petitesse du globe de verre qui l'enfermait; pouvait ètre très rapproché de l'objet pardessus ou par-dessous la platine, ce qui permettait d'éclairer celui-ci

(1) Voir Journal de Micrographie. T. VII, 1883, p. 244. 
directement d'une manière 'très intense. Ces lampes n'exigeaient qu'une force électromotrice de $31 / 2$ volts et un courant de 1 ampère 1/4. Leur puissance lumineuse équivalait à environ une bougie, mais pouvait être poussée au besoin à 2 bougies $1 / 2$ pendant quelques moments.

Ces lampes, pouvaient être fixées sur le microscope à la place du miroir par exemple ou portées par un pied muni de bras articulés qui permettait de donner à la lanipe telle position que l'on roulait au-dessus ou au-dessous de l'objet.

“ L'éclairage électrique par incandescence, dit à ce sujet M. Van Heurck, surpasse tout autre éclairage. Il a la douceur des bonnes lampes à pétrole et montre les détails délicats presque aussi

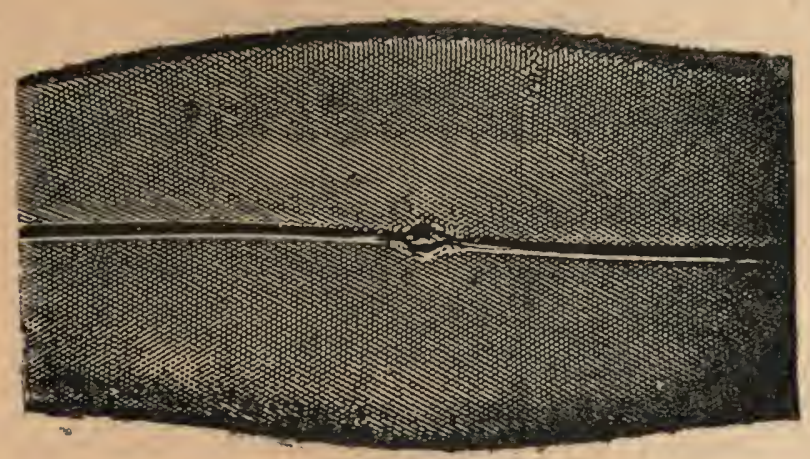

Fig. 91 - Pleurosigma angulatum, résolu et photographiẻ à la lumière électrique par M. H. van Heurck.

bien que la lumière monochromatique. Les stries délicates de $l^{\prime} A m p h i p l e u r a$ et le $19^{\circ}$ groupe de Nobert se voient avec une netteté parfaite."

Dès cette époque, M. H. Van Heurck avait appliqué l'éclairage électrique des lampes Swan à la photomicrographie et arait obtenu d'excellents résultats.

Depuis lors, il a remplacé le pied-porte-lampe par un photophore analogue à celui dont se servent Mir. Hélot et Trouvé pour l'éclairage des cavités du corps humain.

C'est un petit cylindre nickelé A (Fig 90) portant au fond un miroir réflecteur, au milieu une petite lampe à incandescence et au sommet. une lentille condensatrice dont la monture glisse à frottemént doux dans le cylindre, ce qui permet de faire varier la divergence des 
rayons émergents, de les amener au parallélisme et mème, suivant les besoins, à la convergence (1).

Cet appareil est fixé sur un pied autour duquel il peut prendre toutes les inclinaisons et les positions voulues, $\Lambda, \Lambda^{\prime}, \Lambda^{\prime \prime}$, de manière à éclairer le microscope au gré de l'observateur.

Employé comme source de lumière pour la micro-phothographie, le photophore Hélot-Trouvé et Van Heurck donne des résultats excellents, et nous reproduisons le dessin de deux Diatomées résolues et photographiées à l'aide de cet instrument.

Quant à la source d'électricité, elfe peut ètre une simple pile à treuil, de 6 éléments, comme celle que construisent M. Trouvé et

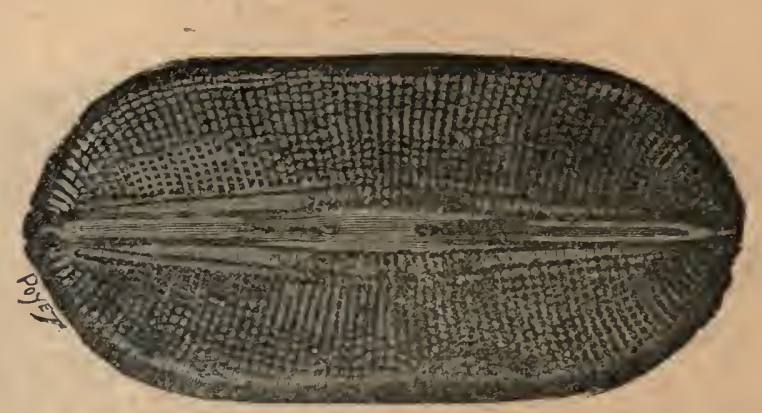

Fig. 92. Navicula fusca, résolu et photographié à la lumière électrique par M. H. van Heurck.

d'autres électriciens et que l'on trouve maintenant couramment dans le commerce.

La photographie est certes un moyen d'étude important pour les Diatomées, mais on comprend que nous ne pourons, relativement à cette question toute particulière, que renvoyer aux traités spéciaux (2).

(1) Voir H. van Hetrck. - Journal de Micrographie, T. X, 1886, p. 29 et suiv.

(2) Voir entre autres: H. vax Hetrck. - Photomicrographie (Journal de Micrographie. T. VII, 1883, p. 257).

H. Viallaxes. - La Photographie appliquée aux éludes d'anatomie microscopique. Paris, in-18, 63 pages, 1886. 


\section{\$. - MICROTOMES}

Microtome à levier, de Hansen. - On s'étonnera peut-ètre de nous voir, dans un livre consacré à l'étude des Diatomées, parler des microtomes, instruments destinés à obtenir des eoupes très minces des objets destinés à l'examen microseopique. Nous le faisons cependant à bon eseient. En eflet, depuis quelques années l'étude, si arriéréc eneore, des Diatomées considérées comme des êtres vivants et non plus seulement eomine de simples frustules silieeux marqués de stries, de côtes ou de perles plus ou moins fines, préoceupe un assez grand nombre d'olsservateurs qui eherehent à pénétrer la structure intime de ces organismes et à élucider les phénomènes biologiques dont ils sont le théatre.

Or, nous sommes aetuellement, en mierographie, dans ce qu'on peut appeler l'ère des coupes. - C'est par l'étude des coupes minces, exéeutées arec une perfeetion ineonnue jusqu'ici et faites méthodiquement. que l'on est parvenu non seulement à reeonnaître d'une manière cxaete la structure histologique des animaux et des plantes, mais encore à suivre, pour ainsi dire pas à pas, certains phénomènes érolutifs qu'il arait été impossible d'analyser alors. qu'on ne disposait que des nétlıodes d'observation in toto, de dissoeiation ou de compression.

Il faut ajouter à la pratique perfeetionıée des eoupes, les progrès de la teelınique, les procédés nouveaux et si nombreux de coloration qui ont permis de caraetériser d'une manière plus complète les éléments histologiques et de reeonnaitre la différeneiation des substanees qui les composent.

Déjä, par l'étude des eoupes de Diatomées, telles qu'on en trouve quelquefois dans certaines roehes, quelque's observateurs, comme MM. J. Deby, W. Printz, Van Ermengem, sont parvenus à élueider des détails de strueture yu'il avait été jusque-là impossible de reconnaitre d'une manière eertaine. I' autres observateurs ont eherché à appliquer la méthode des eoupes aux Diatomées vivantes, et e'est à notre avis une exeellente voic à suivre et qui sera sans doute féconde en déeourertes, en ee qu'elle nous rérélera la strueture intime de l'organisme vivant, la disposition et la nature des matériaux organisés qui eomposent la eellule diatomée, ainsi que les modifieations que subissent ees éléments dans les différentes phases de l'évolution biologique de ces Algues.

La principale cause qui a sans doute empèché jusqu’à présent les diatomistes d'appliquer tous les proeédés délicats de la teelıique moderıe, à eommeneer par la méchode des coupes, est l'extrême 
difficulté qu'on éprouve à pratiquer des coupes sur ces ètres microscopiques. La chose n'est cependant pas impossible et nous arons pu commencer, gràce à cette méthode, des travaux qui malheureusement ne sont pas assez arancés pour pouvoir trouver place dans le présent ouvrage. Néanmoins, les coupes que nous avons faites récemment sur quelques grandes espèces nous ont pernis, par exemple, de vérifier, - et de reconnaitre pour parfaitement exacte, - la disposition de l'endochròme dans ces espèces telle que l'a décrite II. Paul Petit (1)

Il est évident d'abord qu'il ne peut pas être question ici de prendre une Diatomée au bout d'une pince et de la débiter en tranches avec un rasoir. Il faut employer des procédés beaucoup plus délicats et avoir recours aux instruments les plus perfectionnés.

Voici comment on peut procéder :

On prend une certaine quantité d'une récolte de Diatomées que l'on veut étudier, en ayant soin de les débarrasser, par les procédés de nettoyage que l'on connait, de toutes les impuretés et notamment des grains de sable, mème les plus petits, qui feraient déchirer la coupe et ébrécheraient le rasoir. Il est clair d'ailleurs que les espèces ou les frustules les moins silicifiés se prèteront micux à l'opération.

On délaie les Diatomées dans une petite quantité d'une solution de gomme arabique très épaisse, à laquelle on peut ajouter un peu de glycérine pour l'empècher de devenir cassante en séchant. Les Diatoniées restent suspendues dans la solution. On prend alors une forte goutte du mélange au bout d'une baguette de verre et on la dépose sur un morceau de liège ou de moelle de sureau, et on la laisse sécher à l'abri de la poussière.

Quand la goutte est sèche, on peut en déposer une seconde sur la première et même une troisième et une quatrième, mais il n'y a pas avantage, en général, à obtenir une couche trop épaisse.

Lorsque la masse d'inclusion ou d'enrobage est bien sèche, il est quelquefois utile, si les Diatomées sont assez fortement siliceuses, de la durcir encore en plongeant toute la pièce dans l'alcool à $82^{\circ}$, pendant 24 ou 48 heures Quand clle est suffisamment durcie, qu'elle ne cède plus sous la pression de l'ongle, il n'y a plus qu'à opérer des coupes comme on le fait dans les laboratoires d'histologic.

$\mathrm{Au}$ lieu de la solution de gomme, on peut employer, à une douce chaleur, une solution de gomme et de gélatine, ou le collodion, ou la celloïdine, qui donne de très loons résultats et permet de faire des petits blocs tenant un grand nombre de Diatomées en suspension, 
mais les coupes obtenues nous ont paru moins satisfaisantes et sont plus difficiles à traiter ultérieurement.

Pour faire les coupes, on ne peut espérer réussir en opérant à main levée avec un rasoir. Le coup de rasoir donné avec la main n'est pas assez rapide et fort: les Diatomées que la lame rencontre en traversant la masse ont le temps de faire bascule sous la pression du couteau, en raison de la résistance qu'elles opposent, résistance grande relativement à celle de la masse qui les entoure.

Il faut de toute nécessité émployer un microtome, et mème un excellent microtome, parfaitement construit, d'une marche très régulière et armé d'un rasoir à trempe dure. Le rasoir sera, du reste, assez vite émoussé et aura souvent besoin d'ètre aiguisé.

On se sert aujourd'hui beaucoup des microtomes allcmands qui fonctionnent en général bien, mais nous pouvons surtout recommander un excellent instrument français, le microtome à levier, de Hansen, construit par M. E. Deyrolle, à Paris. Le maniement en est infiniment plus commode que celui des instruments allemands; la construction et la marche de l'appareil sont parfaites, et sa solidité extrème.

Ajoutons que cet instrument peut servir dans un laboratoire, a toute espèce de coupes et qu'il fonctionne à volonté dans un liquide ou sur des corps durcis par congélation.

Le microtome de Hansen est à glissement pour le rasoir et à levier pour l'objet. Le rasoir est monté sur le chariot au moyen d'un dispositif nouveau qui lui permet de prendre toutes les inclinaisons possibles.

La pièce portant l'objet est soulevée, après chaque coup de rasoir, par un levier sur lequel agit à l'autre bout une vis micrométrique de $1 / 2$ millimètre de pas, dont la tète porte un cercle divisé en ๖े0 dents; quand, à l'aide d'une petite manivelle, on fait tourner le cercle d'une division, la vis s'abaisse de 1/30 de 1/2 millimètre ou $1 / 100$ de millimètre et comme le bras du levier qui agit sur la pince

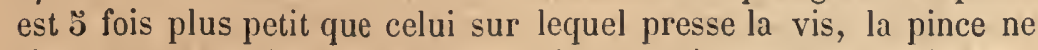
s'élève que de 1/: de 1/100 de millimètre, c'est-à-dire de 1/:ّ00 de millimètre, ce qui représente une coupe extrêmement mince.

S'il était possible de réaliser pratiquement sur les Diatomées des coupes aussi finés, ce qui scrait sans doute très difficile, on voit qu'on pourrait réellement débiter un frustule en tranches, puisqu'il y a des espèces qui mesurent $0^{\mathrm{mm}}, \ddot{\mathrm{O}}$ de long dans lesquelles on pourrait faire deux coupes, et que la plupart ont plusieurs centièmes de millimètres. Mais nous pensons qu'il nest pas nécessaire d'obtenir des coupes aussi minces et qu'il suffit d'avoir une coupe dans un frustule. 
Pour cela, on monte le morceau de liège ou de sureau sur lequel on a déposé les Diatomées enrobées dans la gomme, dans la pinee du microtome, en ayant soin de le fixer solidement sans l'éeraser. On doit, d'ailleurs, avoir eu la précaution de placer la goutte diatomifère sur le liège, de manière qu'on puisse y faire des eoupes perpendiculaires à la surface et non des coupes parallèles ou tangentielles qui réussissent, en général, beaucoup moins bien. En disposant la pièce, comme nous venons de le dire, il suffit de pousser d'un eoup un peu sec le chariot du rasoir, et la lourde lame traverse à la fois la goutte gommeuse et le morceau de liègc qui lui sert de support. Les Diatomées saisies entre le tranchant de la lame et le fond de liège résistant n'ont pas 'le temps de se déplacer et sont traversées par le couperet, qu'on repousse aussitòt cn place.

On fait avancer la roue de ฐ̃ ou 10 dents, selon qu'on a réglé la marche de l'instrument, et l'on donne un seeond coup de rasoir. On obtient ainsi une première coupe régulic̀re qui a $1 / 100$ ou $1 / 50$ de millimètre d'épaisseur. On peut en pratiquer une série d'autres, qui, mème beaucoup plus épaisses, seront fort utiles.

Ces coupes, telles que nous les avons faites, contiennent une partie de liège ou de sureau et une partie de gomme diatomifère. On les place sur des lames de verre dans un peu d'eau; la gomme se ramollit bientôt et l'on peut enlever toutc la portion étrangère. Si l'on couvre d'une lamelle et qu'on examine au microseope, on constate qu'un grand nombre de Diatomées ont échappé à la lame ; mais certaines aussi ont été tranchées et l'on a des coupes de frustules dans toutes les directions. Les deux faces de la préparation présentent des résultats semblables.

C'est dans cet état, alors que la masse d'inclusion conserve sa cohérence, quc l'on peut le mieux étudier la structure des éléments constituants de la eellule diatomée, parce que les frustules gardent leur position. Si l'on ajoutc de l'eau de manière à dissoudre la gomme, presque toutes les Diatomées n'étant plus maintenues par la masse, tombent à plat sur la lame de verre et l'on n'en rencontre plus guère qui présentent leur section à l'observateur. Néanmoins, on peut encore en retrouver quelques-unes et faire agir sur elles les réactifs colorants, diverses couleurs d'aniline, par cxemple, et le picrocarminate d'ammoniaque qui colorc le protoplasma en jaune, le noyau cn rouge, l'endoehròme en brun foneé et laisse les gouttelettes huileuses ineolores. Quand la coloration est produite, on ajoute sur un des bords de la lamelle une goutte de glycérine et l'on aspire du còté opposé avec du papier brouillard. La glycérine pénètre ainsi peu à peu la préparation, que l'on peut dès lors transformer en une préparation persistante. 


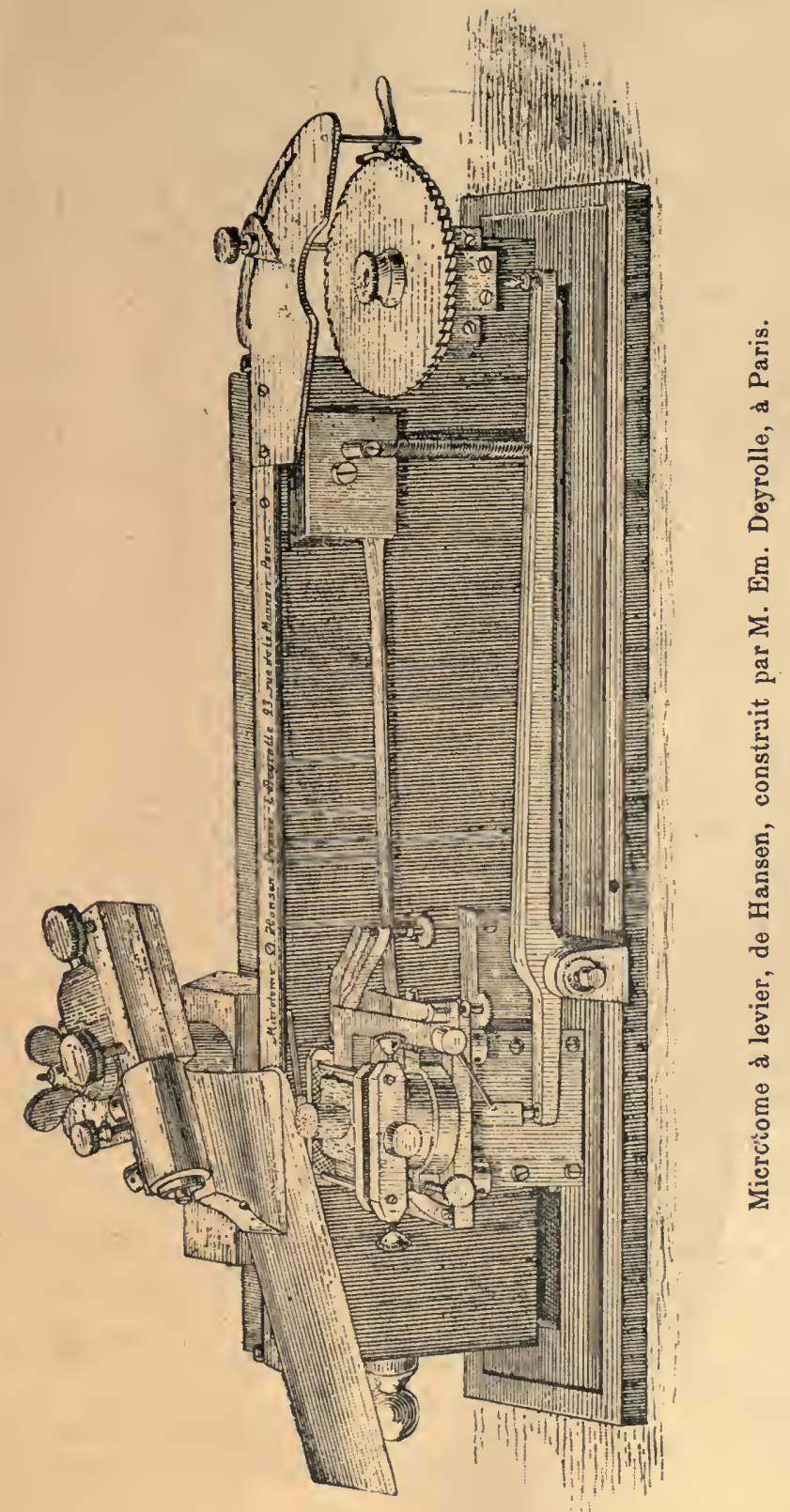


Une très bonne méthode, et mème la meilleure, consiste à fixer d'abord les éléments dans leur forme, par l'acide osmique. On peut exposer les Diatomées. délayées dans un peu d'eau, aux vapeur's d'une solution d'acide osmique à 2 pour 100 ; mais la pénétration des rapeur's à travers la carapace siliceuse est difficile. Il vaut mieux verser sur les frustules, suspendus dans un peu d'eau, quelques gouttes de la solution osmique. On recouvre d'une cloche et, au bout d'une heure, on jette le tout sur un filtre et on lave avec de l'eau distillée pour enlever l'acide. Puis, on reprend les frustules, en ràclant le filtre doucement avec une lame de scalpel. On les met dans la gomme et l'on fait les coupes comme nous l'avons dit.

La fixation par l'acide osmique n'empèche pas la coloration des coupes, sur la lame de verre, par le picrocarminate; on recouvre d'une lamelle et l'on déplace le picrocarminate par la glycérine. La coloration est plus longue à obtenir, mais se produit généralement bien. Les globules huileux sont alors colorés en noir intense et l'on peut constater qu'ils sont ordinairement très nombreux.

On peut, nous l'arons dit, employer pour l'enrobage le collodion ou la celloïdine (qui n'est qu'un collodion solidifié, autrement dit de la pyroxyline; que l'on redissout dans un mélange à parties égales d'éther et d'alcool absolu), mais il faut, dans ce cas, commencer par déshydrater les Diatomées dans l'alcool ordinaire, puis dans l'alcool absolu. Après quoi, on les porte dans le collodion ou dans une solution faible éthéro-alcoolique de celloïdine, où on les laisse une journée. Puis, on les plonge dans une solution sirupeuse de la matière à inclusion, et l'on opère avec celle-ci comme nous l'avons indiqué pour le traitement dans la gomme.

* On réussit ainsi beaucoup moins bien qu'avec la gomme; outre que l'alcool et l'éther dissolvent plus ou moins la matière colorante de l'endochròme et les gouttes huileuses, la masse a moins de cohésion, les Diatomées se déplacent et fuient sous le rasoir; les coupes, que l'on. doit faire avec le rasoir mouillé, s'effritent, se dessèchent et se ratatinent. Ausși, est-on obligé de les faire beaucoup plus épaisses. Pour remédier au manque de cohésion de la celloïdine, on peut faire durcir la masse avant de pratiquer les coupes, en plongeant le liège et la couche diatomifère qu'on y a déposée dans l'alcool à $82^{\circ}$ ou dans le chloroforme pendant 24 ou 48 heures, et mème davantage.

Les coupes sont reçues dans l'alcool et peuvent ètre examinées, montées dans la glycérine, sans qu'il soit besoin d'enlever la masse d'inclusion qui est parfaitement transparente et se laisse pénétrer par la glycérine. On peut mème faire agir dans les mèmes conditions les réactifs colorants.

Le montage de ces coupes dans le baume ne peut, au contraire, se 
faire qu'après traitement par l'essenee de bergamotte. On aura d'ailleurs à mettre en œuvre tous les procédés ordinaires de la teehnique des eoupes. Mais nous devons faire remarquer que les traitements par l'alcool sont loin de conserver dans l'intégrité de leurs formes, de leurs rapports et de leur substanee les éléments si riches en eau qui eomposent la eellule Diatomée, à moins qu'on ne les ait préalablement fixés par l'acide osmique. Dans le cas eontraire, nous préférons le proeédé d'inclusion dans la gomme, auquel ehaeun peut du reste apporter les modifieations que eomportent les divers eas, qui est plus eommode et nous parait montrer suffisamment la disposition intérieure des cellules telle qu'elle est réellement dans la nature. (Voir Pl. IV.)

Nous n'arons pas à entrer dans plus de détails sur ces opérations. Cette méthode d'étude des Diatomées à l'état vivant est eneore peu appliquée, pas toujours applieable, mais e'est une voie nouvelle à explorer qui, nous le pensons, peut amener la résolution de bien des questions eneore à l'état de problèmes.

Malheureusement, elle n'est pas toujours applicable, disons-nous, car beaucoup de Diatomées à carapace très silieeuse résistent complètement au rasoir du microtome. Il faudrait pouvoir les ineorporer dans une masse plus cohérente que la gomme, et l'on éprouverait alors de grandes difficultés à exéeuter les eoupes, à les ohtenir suffisamment minces et à les rendre assez transparentes pour permettre d'en faire utilement l'examen microseopique.

Ajoutons que M. J. Deby est arrivé à faire des eoupes de Diatomées en composant une masse d'inclusion dure, pierreuse, pour ainsi dire, atec des chlorures de zine et de magnésium mêlés aux oxydes des mêmes métaux, constituant ainsi une roche artifieielle dans laquelle il a pratiqué des coupes (1) par les procédés qui servent à faire des coupes minéralogiques.

MII. Prinz et Van Ermengem s'étaient servis de la mème manière de la pierre à eiment du Jutland et de l'argile de Londres qui contiennent des valves de Diatomées fossiles (2).

M: Flogel (3) avait aussi opéré des eoupes de Pleurosigma;

(1) J. DEBY. - Sur la structure microscopique des valves des Diatomées. (Joirn. de Micrographie, T. X, 1886, p. 416 et 467.)

(2) W: Prinz et Van Ermengem. - Recherches sur la stricture de quelques Diatomées comprises dans le Cementstein du Jutland (Ann. Soc. B. de Microscopie, T. VIII, et Journ. de Mícrographie, 1881, p. 100.)

(3) FlogeL. - Untersitchungen ïber die Structur dev Zelluand in c. Gattung Pleurosigma. (Ar: $f:$ Mik. Anat:, VI, 1870:) 
MI. Otto Müller (1), J. D. Cox (2) et II. Van Heurck avaient utilisé des valves brisées de Triceratium et d'autres espèces pour étudier la structure microscopique de ces valves.

Mais on comprend que les études ainsi faites ne peuvent porter que sur des frustules morts, dans lesquels la matière organique est plus ou moins détruite, et par conséquent ne peuvent rien révéler sur l'organisation intérieure de la Diatomée vivante ni sur les phénoménes biologiques qui s'y produisent.

(1) Otto Mulder. - Uber den feineren Bau der Zellwand der Bacillarien, etc. (Arc. f. Physiol., Reichert et Dubois-Reymond, 1871.)

(2) J. D. Cox. - Structure of the Diutom-Shell. (Am. Month, Micr. Journ. 1884.) 


\section{VIII}

\section{CLASSIFICATION}

\section{§ 1. - SYSTEMES DE CLASSIFICATION}

Nous avons dit que plusieurs systèmes de elassification avaient été proposés pour la famille des DIATOMÉES ou DIATOMACÉES ; aujourd'hui, il n'en reste guère que deux en présence; celui que le célèbre diatomiste américain Hamilton Lawrence Smith a établi sur la présence ou l'absence d'un raphé, et celui que le prof. Pfitzer a proposé de fonder sur la disposition de l'endochrôme, système que M. Paul Petit, le plus autorisé de nos diatomistes français, a appliqué développé et complété. C'est cette classification que nous avons adoptée ici et que nous suivons dans la description que nous donnons des principales espèces de Diatomées.

La elassification du prof. H. L. Smith a été adoptée par plusieurs auteurs, notamment par le Dr H. Van Heurek, dans son bel ouvrage: Synopsis des Diatomées de Belgique. Nous en avons déjà indiqué les bases, nous n'y reviendrons done qu'en quelques mots.

M. H. L. Smith divise la fanille des Diatomées en trois sousfamilles.

$4^{\circ}$ Les Raphidées, ayant un vrai raplé, au moins sur l'une des valies.

(Frustules à face valvaire généralement bacillaire, parfois largement ovale, montrant toujours un raphé distinet et des nodules sur l'une des deux valves ou sur toutes les deux. Nodule médian rarement absent ou peu visible. Valves simples ou composécs. Frustules toujours dépourvus de dents, d'épines ou de piquants.)

Cette sous-famille comprend les einq tribus suivantes: Les Crm= BelléEs, les Naviculées, les Gomphonémées, les Achnavthées et les COCCONÉIDÉES.

$2^{\circ}$ Les Pseudo-Raphidées, ayant sur les deux valves, ou au moins sur l'une d'elles, un espace blane simulant un raphé (pseudoraphé). 
(Frustules à face valvaire grénéralement bacillaire, parfois largement ovale ou sub-orbiculaire, présentant souvent des cloisons ou fausses cloisons.)

Cette sous-famille comprend trois tribus : les Fragllariées, les Tarellariées et les Surirellées;

$3^{\circ}$ Les Crypto-Raphidées, n'ayant jamais de raphé ni de pseudoraphé sur aucune des valves.

(Frustules à face valvaire généralement circulaire, sub-circulaire ou angulaire, plus rarement elliptique, ovale ou bacillaire; sourent munis de dents, d'épines ou de piquants.)

Cette sous-famille compte sept tribus : les Ch.tocÉRÉEs, les MÉLOsirées, les Biddulphiées, les Eupodiscées, les Héliopeltées, les Astérolamprées et les Coscinodiscées.

Quant à la classification de M. Paul Petit, ce savant diatomiste, après l'avoir reviséc, complétée relativement à certains genres difficiles, et mise au point, pour ainsi dire, a bien voulu en rédiger, spécialement pour le présent ouvrage, un exposé général, accompagné d'un tableau dichotomique qui la résume tout entière et en rend l'application des plus faciles.

Nous laissons la parole à M. Paul Petit.

\section{§. - CLASSIFICATION}

Pour classer les Diatomacées, alors que les espèces connues étaient en petit nombre, plusieurs systèmes, plus ou moins empiriques, furent mis en usage par Agardh, Ehrenberg, W. Smith, Kützing, Meneghini et Rabenhorst. Le cadre de cet ouvrage ne nous permet pas de faire séparément l'analyse de chacun de ces systèmes. Il suffira de dire que ceux qui ont été employés par la plupart des auteurs indiqués ci-dessus reposent, tantòt sur la forme extéricure des frustules ou des valves, tantòt sur le mode de régétation des Diatomacées, qui vivent libres, ou réunies en filaments, quelquefois portées à l'extrémité d'un stipe ou enfermées dans des masses gélatineuses, ayant la forme d'un tube ou d'une fronde.

En 1861, Ralfs (in Pritchard : Infusoria), donna une classification basée sur la présence ou l'absence du raphé ou des nodules, dans laquelle il faisait ressortir l'affinité existant entre certaines familles. Cette classification fut reprise dix années plus tard par M. H. L. Smith, comme nous le verrons plus loin.

A peu près dans le mème temps, le prof. Grunow (Verhandl. 
der K. K. zool. bot. Gessellschaft, Wien, 1860-1862), faisant la description des Diatomacées d'Autriche, indiquait la délimitation et l'affinité dc plusieurs familles très naturelles.

W. Smith, dans sa classique Synopsis, avait appelé l'attention des observateurs sur la fixité de l'endochrôme, qui se présente tantòt en lames, tantòt en granules. (Synopsis, vol. II, p. xxr.) Cette remarque de W. Smith frappa le prof. Pfitzer, qui cntreprit une étude séricuse du plasma des Diatomacées. Il publia, en 1871, les résultats de scs recherches dans un remarquable travail : Bau und Entwicklung der Diatomaceen. La conséquence de ces recherches fut une nouvelle classification, baséc sur la disposition de l'endochròme dans lc frustule, mais dans laquellc le prof. Plitzer ne rechercha pas assez l'affinité des genres et des grands groupes.

L'année suirante, cn 1872, le prof. H. L. Smith, de Hobart College, à Geneva (E. U.), reprenait, cn la développant, la classification de Ralfs. Ce travail fut publié dans The Lens, qui périt en grande partie dans l'incendie de Chicago; mais il fut plus tard traduit et reproduit par M. Van Heurch, dans son livre Le Microscope, 1878.

Le système mis en usage par M. H. L. Smith a le grave inconvénient d'éloigner les uns des autres des genres dont l'aflinité est manifcste. C'est ainsi que le genre Gephyria, qui, par tous ses caractìres, se rattache aux Achnanthes, cst placé par l'auteur américain près du genre Eunotia, qui n'a rien de commun avec lui, si ce n'est la courburc des frustules. De mème les Licmophora, que la présence de diaphragmes intervalvaires rapproche des Tabellariées, se trouvent placés parmi les Fragilariées, ctc., ctc.

C'est en vérifiant les olscrvations du prof. P(itzer, et en les complétant par l'étude d'espèces marines et d'eau douce, qu'il n'avait pas eues à sa disposition, que nous avons compris tout le parti qu'on pouvait tirer de la forme et de la position de l'endochròme dans le frustulc pour la classification des espèces. Une étude approforidie d'un grand nombre d'espèces nous a permis de saisir l'cnchainement des genres cntre eux et de suivre une chaine non interrompue, partant du genre Cocconeis et aboutissant aux Gaillonellées. C'cst alors que nous arons publié, en 1876 (Bull. de la Soc. de Bot. de France), un Essai de classification naturelle, basé sur la disposition de l'endochròme dans le frustule. Les obscrvations, que nous avons poursuivies depuis cette époque, sont venues confirmer ct corroborer notre manière de voir.

Lorsqu'on observe un grand nombre de Diatomacées, à des époques différentes, et en répétant souvent ces observations, tant pour les espèces marines que pour les cspèccs d'eau doucc, on peut se convaincre, ainsi que W. Smith l'avait indiqué, que l'endochròme 
se présente sous deux états distincts et que, contrairement à l'opinion de M. l'abbé eomte Castraeañe (1), lá disposition de l'endochròme est fixe et invariable chez tous les individus d'une mème especce. On peut observer une légère variation à l'époque de la division de la cellule; mais aussitot après la division, l'endochròme reprend son état normal. Il est en mème temps faeile d'avoir la preuve que la division de l'endochrome lamelleux en petite masse n'est pas, comme le pense II. l'abbé Castraeane, le prélude de la repiroduction, mais que cet état est le signe précurseur et certain de la mort prochaine du firustule.

L'observation permet encore de remarquer qu'il existe un rapport eonstant entre la disposition de l'endochròme et, la forme extérieure du squelette siliceux du frustule, dans toutes les espèees d'un mème genre et, souvent aussi, ehez plusieurs genres ayant entre eux des analogies de constitution ou de développement de l'enveloppe siliceuse.

Nous sommes donc eonduits à formuler les deux principes suivants :

$1^{\circ}$ La disposition interne de l'endochrôme est constante chez tous les individus d'une même espèce;

20 Le rapport clu firustule et de l'endochrôme est fixe et commun à toutes les espèces d'un même genre, et souvent à plusieurs genies ayant entre eux une grande analogie de constitution et de développement dans leur enveloppe silicerese.

Ces deux prineipes permettront de elasser les espèces fossiles, ou celles provenant de sondages profonds, alors que le caractère tiré du plasma fait eomplètement défaut, contrairement à l'opinion émise par MII. H. L. Smith et Van Heurck dans la a Synopsis des Diatomées de Belgique ». Le rapport entre l'endoehròme et l'enveloppe silieeuse est le mème que eelui qui existe en zoologie entre les viscères et le squelette; et ehacun 'sait que les zoologistes ne sont pas embarrassés pour classer à sa place un squelette fossile. Pourquoi les diatomophiles rencontreraient-ils plus de diffieulté?

Il est plus que probable que le rapport entre l'endochròme et l'enveloppe siliceuse s'applique à la reproduction par auxospores; malheureusement, le nombre des observations faites à ce sujet est eneore trop restreint et les observations elles-mêmes ont besoin d'ètre contrôlées, ce qui ne permet pas d'en tirer un caractère ayant une valeur réelle.

(1) Brebissonia, vol. I, p. 75. 
Nous divisons, ainsi que le fait le prof. Pfitzer, la famille des Diatomacées en deux sous-familles :

$1^{\text {re }}$ sous-famille : Endochrôme lamelleux : PLACOCHROMATICÉES.

$2^{\text {me }}$ sous-famille : Endochrôme granuleux : CoCCOCHROMLTICÉES.

Nous sommes d'avis que toute classification naturelle doit ètre basée sur la subordination des caractères. Parmi les caractères, la disposition de l'endochròme dans le frustule et, à son défaut, la constitution du frustule lui-mème, doivent ètre placées en première ligne, puisqu'il existe un rapport constant entre ces deux caractères.

Au second rang nous mettrons la symétrie dans les valves par rapport au grand ou au petit axe, ou la dissemblance des valves d'un mème frustule. Puis, viennent les caractères fournis par la présence ou l'absence de diaphragmes intervalvaires, ainsi que ceux qui proviennent du raphé et des nodules; enfin les caractères extérieurs : carène, ailes, striation, état des valves, forme et aspect extérieur. La distinction des espèces, fournie par les états frondeux, stipité, filamenteux et tubuleux, est de peu de valeur, car ainsi que M. H. S. Smith l'a constaté (et nous sommes d'accord avec lui), ces caractères sont fugitifs et accidentels, et dépendent surtout d'influences extérieures.

J'ai maintenu le genre Stauroneis, qui a été réuni par quelques auleurs au genre Navicula, parce qu'à l'état vivant, sans voir les stries, on peut toujours distinguer un Stauroneis d'un Navicula. Chez le genre Navicula, la lame d'endochròme est lisse sur le bord, tandis que chez tous les Stauroneis elle est ondulée, de mème qu'elle est dentelée dans le genre Amphipleura.

$1^{\text {re }}$ sous-famille : PLACOCHROMATICÉES.

Frustules munis d'un endochrôme lamelleux.

$1^{\text {e }}$ Tribu. - Achranthées.

Cette tribu, telle que l'a établie le prof. Grunow, moins le genre Rhoïcosphenia que nous rattachons aux GonpHosÉméEs, comprend les Cocconéidées et les Achnanthées de M. le prof. Pfitzer.

Elle renferme les Diatomacées qui ont les frustules cintrés ou courbés en genou, et formés de deux valves dissemblables, dont l'une est convexe et l'autre concave; cette dernière seule porte 
un nodule ou un stauros (1), ou une striation différente de la valve convexe
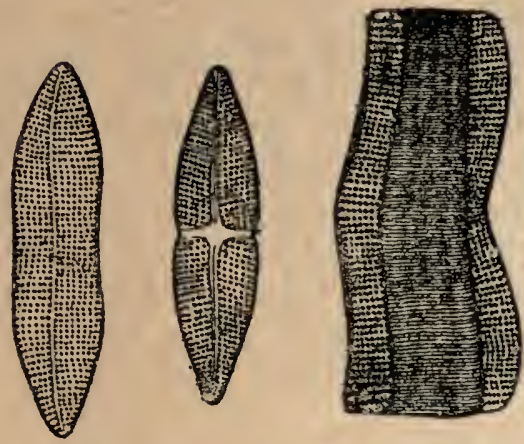

Fig. 93 - Achnanthes brevipes, C. Ag.

L'endochrôme est constitué par une seule lame très épaisse, placée sur la face interne de la valve convexe. Sur le bord de la mer, j'ai pu vérifier la disposition de l'endochròme chez l'A chnanthes longipes, Ag. ; à l'ètat frais, cette espèce rentre dans la loï commune, bien que le D. Pfitzer $(l . c .$, p. $8 \Xi$ ) prétende qu'elle renferme un endochròme composé d'une grande quantité de petits fragments. On observe, en effet, quelquefois cette disposition de l'endochrôme, mais seulement quand les Achnanthes sont depuis quelque temps hors de la mer, attendu que leur plasma s'altère très rapidement.

Cette tribu renferme les genres suivants :

Cocconeis (Ehr).

Cyclophora (Castr).

Cymbosira (Kütz). Achnanthes $(\mathrm{Ag})$.
Gephyr:ia (Arnott). Eupleuria (Arnott). Rhoïconeis (Grun).

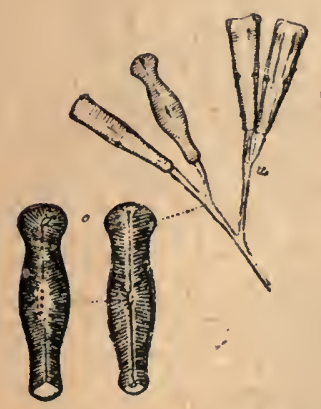

Fig. 94. - Gomphonema geminatum, Ag.

(1) On appelle stauros une surface lisse au niveau du nodule médian. 


\section{ge Tribu. - GoMphosémÉEs.}

Cette tribu comprend les Diatomacées à firustules cunéiformes, munis de ralies cunéiformes symétriques seulement par rappori au raphé, qui possède un nodule central et deux nodules terminaux, excepté dans le genre Rhoïcosphenia, oủ les deux valves cunéiformes sont dissemblables et courbées en genou, comme dans la $1^{\text {re }}$ tribu. C'est ce genre qui fait le passage, puisqu'il a le frustule siliceux des Achrasthées, qu'il est cunéiforme et qu'il possède l'endochrôme des GoMpHoxÉMÉEs : une seule lame reposant par le milieu sur l'un des còtés de la zone et recouvrant les deux valves. (Fig. 94.)

Genres :

Rhoïcosphenia (Grun).

Gomphonema (Ag).

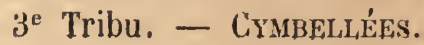

Cette tribu comprend les Diatomacées dont l'endochrôme est formé d'une scule lame reposant par le milieu, tantôt sur la partie la plus concave, tantôt sur la partie la plus convexe, suivant les espèces. Les frustules sont formés par des valves cymbiformes ou cintrées, symétriques seulement par rapport au plus petit axe;

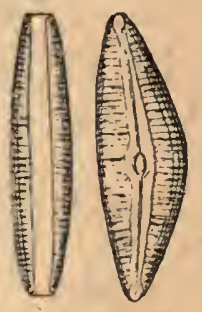

Fig. 95. - Cymbella Ehrenbergii, Kz.

celles-ci sont munies d'un raphé et d'un nodule central, avec deux nodules terminaux.

Genres :

Cocconema (Ehr).

Encyonema (Kütz).

Amphora (Ehr.)

Epithemia (Bréb).

Cymbella (Ag). 
4e Tribu. - Naviculées.

Dans eette tribu, les frustules ont les valves symétriques par rapport au grand axe, qui peut ètre droit ou sigmoïde; celles-ci sont naviculées ou lancéolées, souvent elliptiques ou panduriformes, munies d'un raphé et d'un nodule central, qui manque rarement, et de deux nodules terminaux. L'endochrôme est formé de deux lames séparées, reposant chacune par le milieu sur la zone. Le bord de l'endochròme à l'intérieur du frustule est uni ehez les Navicula;
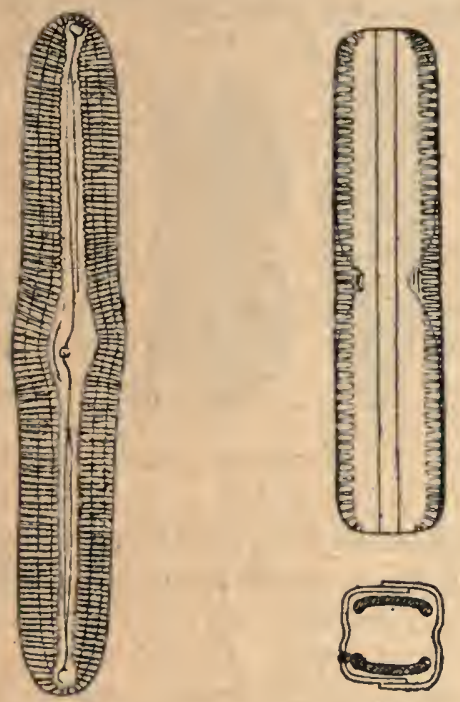

Fig. 96 - Navicula major, Kz.

ondulé chez les Stuuroneis et dentelé chez l'Amphipleura. Le passage aux Cynbelúées a lieu par le Navicula sphcerophora, Kütz, dont l'endochròme est disposé comme chez les Cymbellées, et dont les valves présentent un manque de symétrie, par suite de l'absence de striation sur l'un des còtés du nodule central.

\section{Genres :}

Navicula (Bory).

Schizonema (Ag).

Brebissonia (Gruin)

Mastogloia (Thw).

Stigmaphora (Wall).

Stictodesmis (Grev).

Diadesmis (Kütz).
Stauroneis (Ehr).

Scoliopleura (Grun).

Pleurosigma (W. Sm).

Donkinia (Ralfs).

Toxonidea (Donk).

Berkeleya (Ehr).

Amphipleura (Kütz). 


\section{$5^{\text {e }}$ Tribu. - AmphiprorÉEs.}

Les AMphiprorÉEs ne forment en quelque sorte qu'une sous-tribu des NaviculÉEs. - La disposition de l'endochrôme est la même chez la plupart des espèces, mais la présence de carènes à la surface des valves et la torsion des valves dans un grand nombre d'espèces établissent la différenciation.

Cette tribu forme le passage des Naviculées aux Nitzsc̉híes. Voici comment on peut s'en rendre compte: si l'on considere l'Amphiprora paludosa, W. Sm., on remarque que ses valves sont disposées en 8 de chiffre, mais torducs de tclle sorte que la moitié

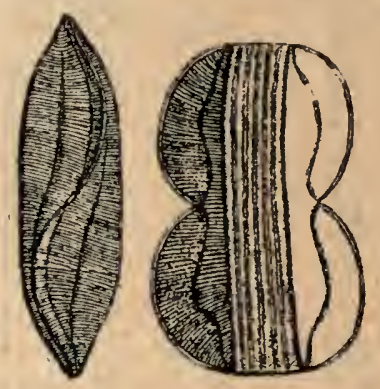

Fig. 97. Amphiprora alata, Ehb.

supérieure est à angle droit avec l'inférieure; de plus, la valve es, tellement pliée sur la carène, qu'il cxiste fort peu de place entre les deux còtés de la zone. On comprend alors que les deux lames d'cndochrome, pressées l'une contre l'autre, se soudent entre elles et que l'œil ne percoive plus qu'une seule lame, disposition qui se rencontre chez les NitzschiéEs.

Genre : Amphiprora (Ehr).

\section{$6^{\text {e }}$ Tribu. - NitzschiÉES}

Les frustules, dans cettc tribu, offrent des formes très variées; les valves sont munies d'une carène ponctuée et l'endochrôme est formé d'une seule lame portant au centre une ouverture elliptique.

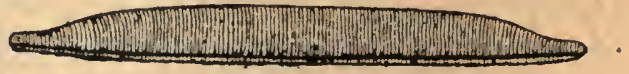

Fig. 98. - Nitzschia vivax, W. Sm.

L'endochrôme se présente sous trois dispositions différentes: $1^{0}$ lorsque les parties opposées de la zone sont resserrées au ipoint 
de laisser fort pcu d'espace cntre elles, l'endochròme ne peut former qu'une scule lame traversant en diagonale le frustule, d'une carène à l'autre; $2^{\circ}$ la lame d'endochròme étant très petite se trouve complètement séparéc en deux parties par l'ouverture centrale; $3^{\circ}$ enfin la lame d'endochròme rcpose sur l'un des cótés de la zone et recouvre les deux valves adjaccntes. Par leurs valves munies de carèncs, les NitzschiÉes se rapprochent des AurphipRoRḱes; d'autre part, certaines formes du genre Tryblionella se rapprochent beaucoup des Cymatopleura, qui font partie des SuriRELLÉEs.

\section{Genres :}

Amphiprora (Ehr).

Nitzschia (Hass).
Bacillaria (Gmel).

Tryblionella (W.Sm).

\section{$7^{e}$ Tribu. - Surirellées.}

Cette tribu comprend les espèces à frustules dont les.valves, sans nodulcs, sont semblables et symétriques par rapport àu grand axe ; elles ont des ailes sur les bords; les frustules sont souvent contournés comme une selle de cheval. L'endochrôme est formé dc deux lames reposant sur les valves. On peut établir le rapprochement de cctte disposition de l'endochròme avec celle du $3^{\text {e }}$ groupe des Nízzschí́Es, en supposant que l'ouverture centrale s'est étendue dans le sens de la longueur jusqu'à séparer l'endochròme en deux parties recouvrant chacune une valve.

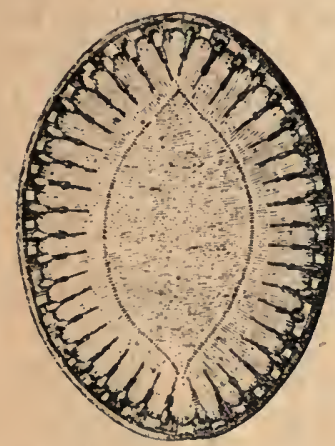

Fig. 99. - Surirella fastuosa. Fhb.

Genres :

Cymatopleura (W.Sm). Campylodiscus (Ehr.)
Surirella (Turpin). Podocystis (Kütz) 
$8^{\mathrm{e}}$ Tribu. - SYNÉDRÉEs.

Chez les Srxédríes, l'endochròme est formé par deux lames reposant sur les valves; il est souvent dentelé sur les bords ou divisé en lanières courtes. Les frustules ont la forme de longs bàtonnets; les valves, symétriques par rapport au grand axe, n'ont pas d'ailes

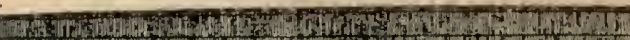

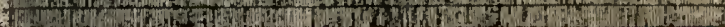

Fig. 100. - Synedru fulgens, W. Sm.

ni de nodules; elles sont striées transversalement et portent une ligne blanche médiane ou pseudo-raphé; on voit quelquefois un large pseudo-nodule au milieu de la valve. On observe chez certaines espèces un élargissement seulement à l'une des extrémités de la valve (Asterionella).

\section{Genres:}

Stanrosira (Ehr).

Thalassiothrix (Clev).

Toxarium (Bail).

Asterionella (Hass). Synedira (Ehr.)

\section{$9^{\text {e }}$ Tribu. - Eunotrées.}

Dans cette tribu, les lames de l'endochiôme reposent par leur milieu sur les valves et recouvrent les côtés adjacents de la zone; elles sont partagées vers le milieu de la zone par un sillon profond s'étendant perpendiculairement jusqu'à la valve. Les frustules rectangulaires sont souvent réunis en filaments rubanés; les ralves sont cintrées, symétriques par rapport au petit axe, quand elles ne sont pas renflés seulement à l'une des extrémitées.

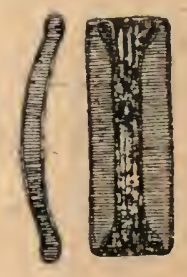

Fig. 101. - Eunotia gracilis, Rab.

Les EunotiéEs se rapprochent, par la forme des frustules, des espèces de Synedra qui ont les valves cintrées. 


\section{Genres :}

Eunotia (Ehr)

Himantidium (Elr). Pseudo-Eunotia (Ehr).
Amphicampa (Ehr).

Clarularia (Grev).

Actinella (Grev).

\section{2e sous-famille : COCCOCHROMLTICÉES}

Frustules munis d'un endochröme granuleux.

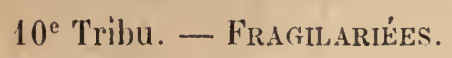

Dans cette tribu, l'endochròme se présente en petites lames séparées ou en granules distincts et épars. Les frustules sont rectangulaires, très rarement cunéiformes, formant des rubans ou adhérant l'un à l'autre par des angles alternants et prenant une forme en zig-zag, ou très rarement en spirale (Meridion); les valves
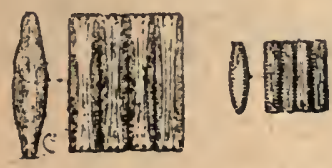

Fig. 102. - Fragilaria virescens ot $F$. mutubilis

ont une forme linéaire allongée, elles sont quelquefois comprimées, lancéolées, très rarement cintrées ou cunéiformes; elles portent des stries fines ou des ponctuations; elles n'ont ni raphé, ni nodules ni ailes, ni carènes.

Cette tribu se rattache d'une part à la tribu des Eusotí́es, par le genre Campylosira, dont les valves sont cintrées, et par le Fragilaria ischaboensis (Grun) et le Fragilaria dubia (Grun), à la tribu des Plagiogramáes.

\section{Genres :}

Campylosiva (Grun).

Cymatosira (Grun).

Raphoneis (Ehr).

Terebraria (Grev).
Meidion (Agardh).

Diatoma (D. C.)

Denticula (Kütz).

Fragilaria (Lyngb).

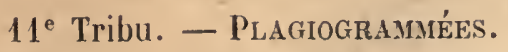

L'endochrôme est constitué par des granules épars; les firustules sont rectangulaires, souvent réunis en filaments rubanés; les 
ralves sont elliptiques, souvent contractées, munies de ponctuations fortes, parfois carrées; on trouve aux extrémités des valves toujours des espaces blancs ou pseudo-nodules, et au centre un ocellus ou un stauros.

Cette tribu, par l'aspect de ses valves et ses frustules rectangulaires, a beaucoup d'affinité avec la tribu des TrachYsPHÉNIÉEs.

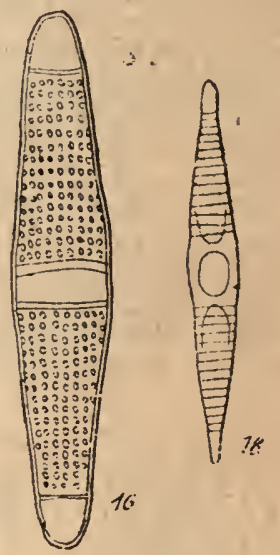

Fig. 103 - Plagiogrämma Antillurum et Pl. attenuatum

Genres : Dimeregramma (Ralfs), Omphalopsis (Grev). Glyphodesmis (Grev), Plagiogramma (Grev).

$12^{\mathrm{e}}$ Tribu. - Trachysphéní́es.

L'endochrôme n'a pas encore été observé dans cette tribu. Les frustules sont rectangulaires, rarement cunéiformes; les ralves sont toujours cunéiformes, portant de fortes ponctuations, souvent carrées, quelquefois remplacées par de larges fentes submarginales, en forme de boutonnières.

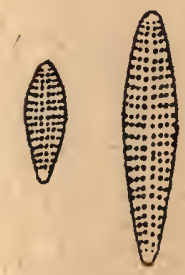

Fig. 104. - Trachysphenia unstralis. Gv.

La disposition des valves en forme de coin constitue dans cette tribu le passage des Plagiogrammées aux Licmophorées. 


\section{Genres :}

Trachysphenia (P. Petit). Sceptroneis (Ehr).
Opephora (1) (P. Petit). Peronia (Bréb. et Arnott).

\section{$13^{e}$ Tribu. - LICHOPIORÉES}

L'endochioime est granuleux, épars sur les cordons de plasma. Les frustules sont cunéiformes, largement arrondis à la partic supéricure; ils sont portés sur des pédicelles gélatineux, qui sont ordinairement ramifiés; les valves sont cunéiformes, fincment striées et portent une ligne médiane sans nodules. Les caractères

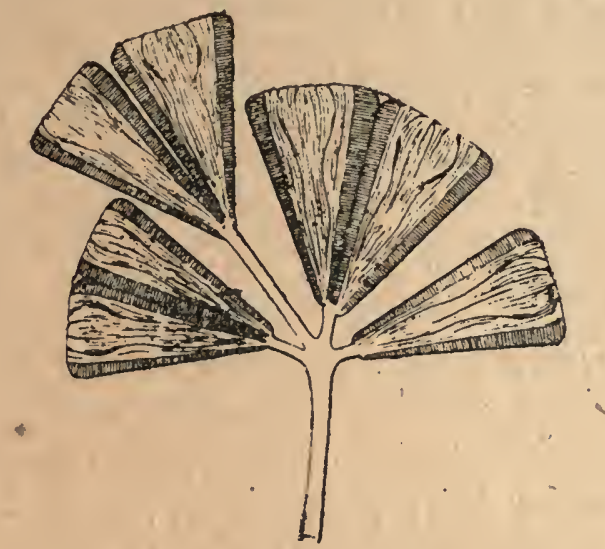

Fig. 105 - Licmophora paradoxa, C. Ag.

distinctifs de cette tribu sont: $1^{\circ}$ les frustules et les valves cunéiformes; $2^{\circ}$ la présence, entre les valves primaires, d'un ecrtain nombre de diaphragmes ou de faux diaphragmes. Ce dernier caractère établit l'affinité de cette tribu avee les TabellariéEs.

Genres : $1^{\text {re }}$ section : diaphragmes rudimentaires :

Podosphenia (Ehr). Licmophora (Ag).

$2^{\text {e }}$ section : deux diaphragmes :

Climacosphenia (Ehr).

$14^{\circ}$ Tribu. - TabellariÉEs.

L'endochiome, dans cctte tribu, est granulcux, tantòt épars, tantòt disposé en lignes rayonnantes autour du noyau; les frus-

(1) Genre nouveau créé (Diat. du Cap. Horn, 1887) pour réunir : Fragilaria pacifica (Grun). - Fragilaria Schwariii(Grun). - Meridion marinum Greg). - Raphoneis australis (H. L. Sm.) - P. P. 
tules sont reetangulaires; les valves sont elliptiques, lancéolées ou linćaires, jamais eunéiformes; souvent renflées au centre et par-

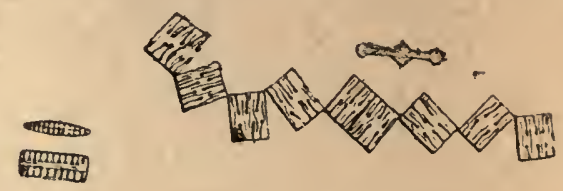

Fig. 106. - Tabellaria flocculosa, Roth.

fois aux extrémités, elles sont finement striécs. Entre les valres primaires se développent un nombre plus ou moins grand de diaphragmes ou septa. Cette tribu comprend les genres suivants :

Genres: $1^{\text {re }}$ seetion: Frustules munis de deux diaphragmes:

Diatomella (Grev). Grammatophora (Ehr).

$2^{\mathrm{e}}$ section: Frustules munis de plus de deux diaphragmes :

* Endochrôme granuleux disposé en lignes rayonnantes :

Rhabdonema (Ehr). Striatella (Ag).

* * Endochrôme granuleux disposé sans ordre :

Tessella (Ehr).

Tetracyclus (Ralts).

Biblarium (Ehr).

Gomphogramma (Braun).
Tabellaria (Ehr).

Hyalosira (Kütz).

Attheya (West).

\section{$15^{e}$ Tribu. - RHzosoléxiées}

L'endochrome est constitué par des granules épars, dans cette ribu qui eomprend les Diatomacées à frustules composés de lames

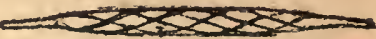

Fig. 107. - Cylindrotheca gracilis, Grun.

silieeuses très nombreuses, soudées par leurs boids en un tube cylindrique terminé par deux valves en forme de coiffes coniques armées de soies ou d'éperons terminaux.

Par ses valves armées de soies, cette tribu, composée jusqu'ici de deux genres, se rattache aux TABELLARIÉEs par le genre Attheya et eneore par ses frustules composés de lames siliceuses. 


\section{Gentes :}

Rhisosolenia (Ehr). Cylindrotheca (Grun).

$16^{\mathrm{e}}$ Tribu. - Chletocḱrḱes.

L'endochiône est sous forme de très gros granules, au moins dans les espèees qui ont été obscrvées à l'état vivant; les frustules sont lisses ou finement ponetués, simples ou réunis en filaments, armés de longues soies ou de piquants; les valves, dans les espèees à longues soies, sont ovales ou presque circulaires et $\mathrm{sem-}$ blables; dans les espèees non filamenteuses, les, valves.sont dissemblables, renflées ou lobées, souvent munies de piquants ou d'autres appendices. Cette tribu, par ses espèces non filamenteuses, offre de grandes affinités arec les BidDclphí́es ; le genre Syringidium (Ehr) présente une assez grande analogie de forme dans les frustules avec le genre Hemiaulus, Ehr., appartenant aux BiddutPHIÉEs.

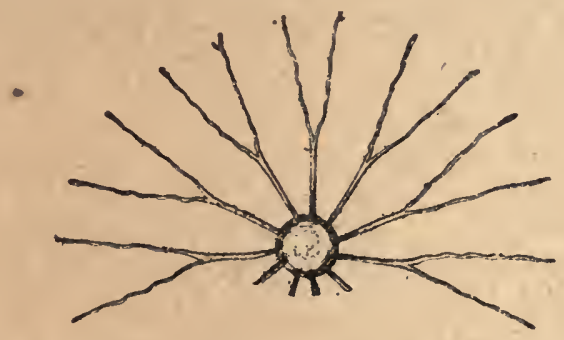

Fig. 108. - Bacteriustrum vuriuns, Lauder.

${ }^{\text {re }}$ section : Espèces à frustules réunis en filaments à articles semblables.
Bacteriastrum (Shad).
Chatoceros (Ehr).

Qe section : Espêces à frustules réunis en filaments; à artieles alternants et à valves dissemblables armées de soies, de cornes, d'aiguillons ou d'épines :

Dicladia (Elı).

Goniothecium (Ehr).

Hercotheca (Ehr).

Periptera (Elir).

Pterotheca (Grun).

Trochosira (Kitt).
Systephania (Ehr).

Cladogramma (Ehr).

Stephanogonia (Ehr).

Syndendrium (Ehr).

Syringidium (Ehr). 


\section{$1 \bar{i}^{\mathrm{e}}$ Triju. - Bindelunés.}

Dans cette tribu l'endochrome est granuleux, disposé en lignes rayonnantes; les firustules sont simples, très polymorphes, munis ou non d'appendices en forme de cornes ou de soies; les valves ont généralement des formes géométriques régulières, du triangle au

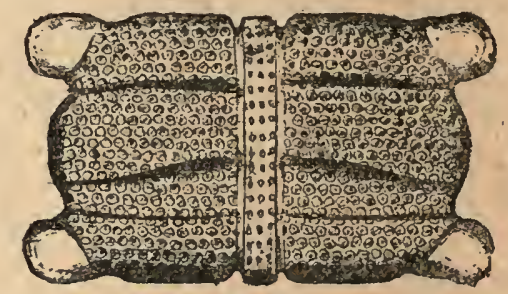

Fig. 109.'- Biddulphia pulchella, Grun.

polygone; elles sont rarement elliptiques, mais jamais complètement discoïdes. Cette tribu a, comme nous l'avons dit, des affinités avec les ChetocÉrÉes par le genre Hemiaulus, et avec les Etpodiscée par le genre Cerataulus.

\section{Genres :}

Hemiaulus (Ehr). Anaulus (Ehr). Porpeia (Bail). Janischia (Grun). Terpsinoë (Ehr). Isthmia (Ag).

Eucampia (Ehr). Ditylum (Bail). Triceratium (Ehr). Amphitetras (Ehr). Biddulphia (Grun). Cerataulus (Ehr).

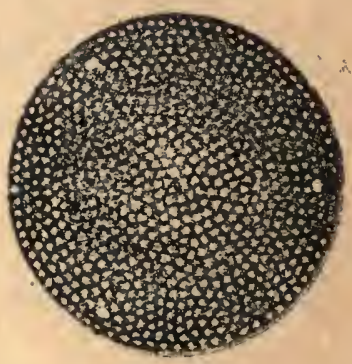

Fig. 110. - Eupodiscus Argus, lihb.

$18^{\mathrm{e}}$ Tribu. - EupodiscÉEs.

Dans cette tribu l'endochrome est épars; les frustules sont simples et les valves discoïdes ou lunulées, munies d'ocelli sail- 
lants, de tubercules, ou sculement d'un nodule submaiginal; la surface des valves est celluleuse, granulée, ou porte des sillons ou rayons moniliformes, ou des stries en forme de barbes de plume. Cette tribu, par le genre Autiscus, se rattaclie aux Biddulphí́es, et par le genre Actinocyclus à la tribu des HÉLiopeltées.

\section{Genres :}

Auliscus (Elır) (Bail).

Monopsia (Gror. et Si).

Pseudauliscus (Leud. F).

Huttonia (Grov. et St).

Eupodiscus (Ehr).

Hemidiscus (Wall).

Euodia (Bail).
Roperia (Grun).

Eunotogramma (Weiss).

Cestodiscus' (Grev).

Craspedoporus (Grev).

Aulacodiscus (Ehr).

Actinodiscus (Grev).

Actinocyclus (Ehr).

\section{$19^{\text {e Tribu, - HÉLIOPELT'ÉES }}$}

L'endochròme est formé de granules petits, épars sur les cordons du plasma; les firustules sont simples et les valves sont discoïdes, quelquefois ondulécs, divisées en segments plus ou moins nombreux, a.ternativement sombres et éclairés; on rencontre souvent un omhilic hyalin au centre et des épines submarginales, différemment placées suivant les genres.

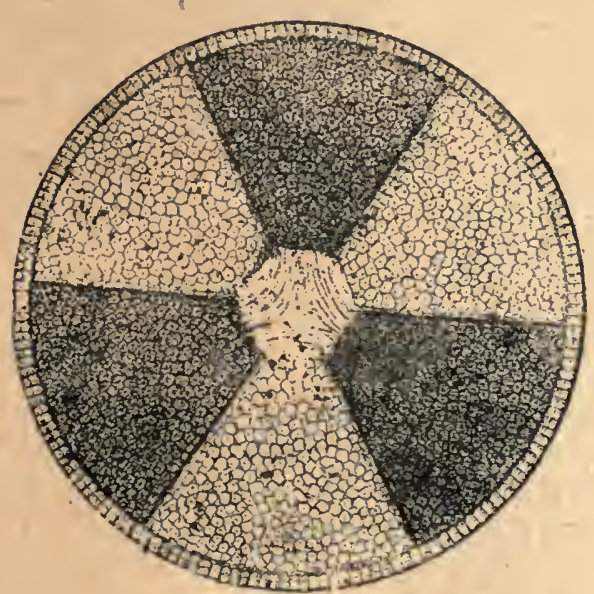

Fig. 111. - Actinoptychus undulalıs, Ehb.

Le genre Actinoptychus, qui forme le passage de cette tribu à celle des Eupodiscḱes, se distingue du genre Actinocyclus par la présence de segments bien marqués sur les valves. 


\section{Genres :}

Actinoptychus (Ehr).

Debya (Pantoc).

Polymyxus (Bailer).

Heliopelta (Ehr).
Halionix (Ehr).

Truania (Pantoc).

Lepidortiscus ( 0 . Wit).

\section{0 'Tribu. - AstÉrolaniries.}

Cette tribu, dont nous n'avons pas encore réussi à observer l'endochròme, renferme des Diatomacées à fiustules dont les valves discoïdes ou orbiculaires présentent des compartiments marginaux aréolés, plus ou moins nombreux, séparés par des sillons hyalins. qui partent d'une aire hyaline centrale, au milieu de laquelle se. trouve souvent un ombilic allongé.

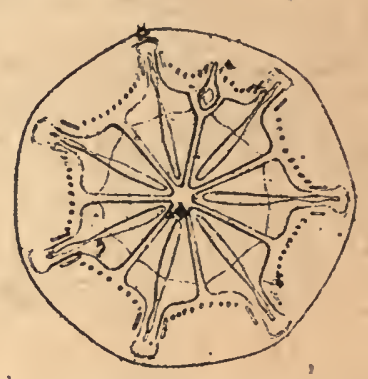

Fig. 112. - Asterolampra Weissflogii, Gr.

\section{Genres :}

Asteromphalus (Ehr). Asterolampra (Ehr).

Asterodiscus (Johnson).

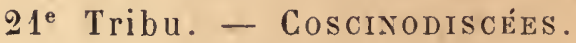

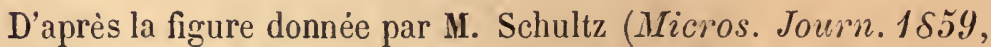
Pl. II, f. 13), du Coscinodiscus centralis (Ehr) vivant. et sur l'affirmative de Borscow (Sussw. Diat. des S. E. Russlands), l'endochrôme des CoscinodicÉEs peut être considéré comme granuleux, ainsi que celui des autres Diatomées discoïdes.

Les frustules sont simples, à valves discoïdes, rarement elliptiques ou réniformes, leur surface est ạéolée ou granulée; elles présentent parfois des couronnes concentriques de texture différente et rarement des dents ou des petites épines submarginales.

Cette tribu a de grandes affinités avec les tribus précédentes par 
la texture de ses valves; par le genre Dictyomyxis, elle passe à la tribu des XasthiopyxidéEs.

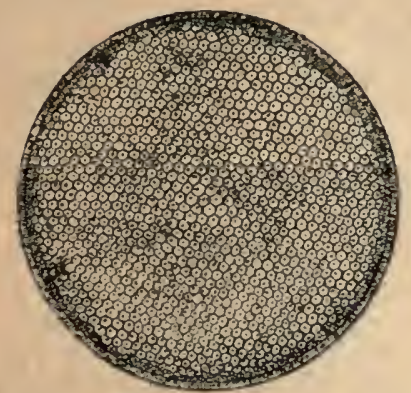

Fig. 113. - Coscinodiscr's leptopus, Grun.

Genres :
A.achnoïdiscus (Deane).
Craspedodiscus (Elır).
Coscinodiscus (Ehr).
Porodiscus (Grev).
Ileterodictyon (Grev).
Dictyopyxis (Ehr).
Brightwellic (Ralfs).

\section{2" Tribu. - XanthiopyxidÉEs}

Dans cette tribu les frustules sont simples et les valves discoïdes ou elliptiques, fortement bombées, non celluleuses, mais chargées d'aiguillons ou d'épines parfois bifurquées. Cette tribu se rattache d'une part aux Coscriodiscées par le genre Creswellia, et aux GaIllonellées pår le Skeletonema mirabile (Grün).
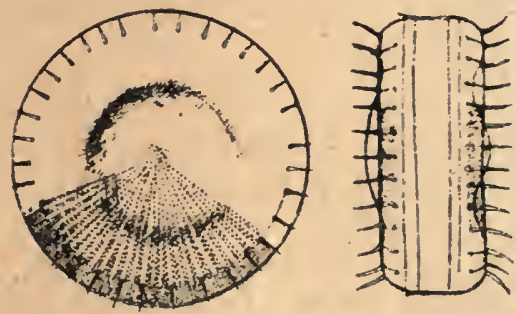

Fig. 114. - Stephnodiscus, Niagarce, Ehb.

\section{Genres :}

Ciesuellia (Grev). Stephanopyxis (Ehr). Stephanodiscus (Ehr). Pyxidicula (Ehr).
Xanthiopyxis (Ehr). Strangulonema (Grev). Skeletonema (Grun). 


\section{$23^{e}$ Tribu. - Gahlionelitées.}

Dans cette tribu l'endochròme est épars à la surface interne des frustules, il est souvent sous forme de forts gros granules anguleur. Les firustules sont. cỵlindriques ou globuleux, simples ou réunis en filaments eylindriques; les valees sont circulaires, quelquefois i

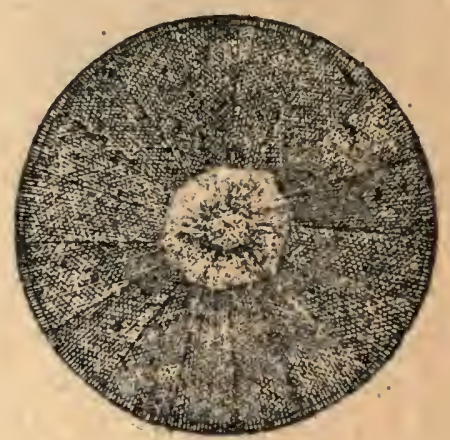

Fig. 115. - Hyalodisous stelliger, Bailey.

ponetuations rayonnantes, d'autres fois granuleuses, eelluleuses ou ponetuées; on remarque, chez certaines espèces, des épines à la surface des valves, comme dans le Gaillonella setosa (Greville), espèee qui a une grande affinité avee les XaxthiopyxidéEs.

\section{Genres :}

Gaillonella (Bory).

Pantocsekia (Grün).

Hyalodiscus (Ehr).
Podosira (Elir).

Cyclotella (Kütz).

C'est avee une certaine hésitation que nous rangeons le genre Hyalodiscus parmi les Galloxeldées. Son endochròme, disposé en étoile à quatre segments, semble devoir l'éloigner de eette tribu, bien que la forme et la disposition de ses valves paraissent l'y rattacher. Ce genre a besoin d'être étudié plus complètement sur les frustules vivants. 


\section{DEUXIEME PARTIE}

\section{DESCRIPTION DES PRINCIPALES ESPÈCES}





\section{IX}

\section{DESCRIPTION DES PRINCIPALES ESPECES}

$1^{\text {re }}$ sous-famille : PLACOCHROMATICÉES

Endochrôme dispose en lames ì la surface du piotoplasma.

$$
1^{\text {Pe }} \text { TRIBU }
$$

\section{ACHNANTHÉES}

Les Achranthées ont un endochròme formé d'une seule lame très épaisse, doublant la face interne de la valve convexe, qu'on appelle aussi valve supérieure ou dorsale. (Fig. 130, 1.)

Ces espèces ont, en effet, les deux valves dissemblables, et de plus, le frustule étant plus ou moins cintré ou plié en genou, lorsqu'on le regarde par la face connective, l'une des valves est convexe et l'autre concave. - La valve concave ou inférieure présente un raphé avec un nodule médian et deux nodules terminaux. La face convere ou supérieure n'a qu'un pseudo-raphé, sans nodules.

Les frustules, indépendamment de la courbure plus ou moins marquée qu'ils présentent, ont, à plạt, une forme naviculoïde, quelquefois elliptique ou mème presque discoïde. Ils sont parfois resserrés au niveau du petit axe, en forme de semelle.

Quelquefois, les frustules restent réunis en séries portées par un pédicelle hyalin.

M. P. Petit classe dans cette tribu les genres :

$1^{\circ}$ Cocconeis, Ehb; $2^{\circ}$ Cyclophora, Castr.; $3^{\circ}$ Cymbosira, Kz; $4^{\circ}$ Achnanthes, Ag.; $\check{5}^{\circ}$ Gephyria, Arnott; $6^{\circ}$ Eupleuria, Arn.; $7^{\circ}$ Rhoïconeis, Grün.

Cocconeis. - Ce genre a les frustules un peu cintrés, quelquefois presque plats, ou un peu gonflés en forme de lentille plan-convexe. Vus par la face valvaire, ils sont elliptiques, assez larges et même quelquefois discoïdes, en bouclier. Les stries, ponctuées, ont une directon généralement transversale; mais celles qui sont au-dessus 
du petit axe se redressent par leur's extrémités vers le póle supérieur de l'ellipse, et celles qui sont au-dessous de cet axe s'infléchissent vers le pòle inférieur, imitant ainsi la disposition des parallèles audessus et au-dessous de l'équateur sur une carte géographique mappemonde.

Ce sont, en général, de petites espèces, qui vivent libres ou collées à plat, comme des parasites, sur les plantes aquatiques.

Le plus commun de tous est le $C$. pecliculus, qu'on trouve appliqué sur presque toutes les algues l'eau douce et mème des eaux saumàtres, comme une punaise, qu'il rappelle un peu par sa forme et sa couleur. Il est fortement courbé. La valve inférieure, qui présente raphé et nodules avec quelques còtes sur les bords, est finement striée de lignes ponctuées transversales, arquées autour des pòles de l'ellipse comme centre: (16 à 17 stries dans 1 centième de mm., V. Heurck).

Sur la face supérieure, on roit un large pseudo-raphé, accompagné, de chaque còté, de lignes ondulées hyalines, qui coupent les stries perpendiculairement. Cette espèce mesure de 1 1/2 à 3 c. de $\mathrm{mm}$. de longueur.

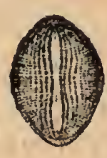

(le

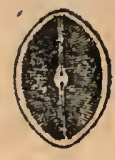

b

Fig. 116. - Cocconeis pediculus, Ehb.

$a$ valve supérieure; $b$ valve inférieure.

Le $C$. placentula, Ehb., commun aussi dans les eaux douces, a à peu près la mème forme, un peu plus arrondie par ses deux extrémités; sa valve inférieure est bordée d'une zone marginale formée de stries à direction rayonnante, ponctuées, plus espacées que celles de la partie centrale ( 1 ă dans $1 \mathrm{c}$. de $\mathrm{mm}$.). Cette zone est séparée de la partie centrale par une ligne ou cadre elliptique hyalin. La partie centrale a 22 stries dans $1 \mathrm{c}$. de $\mathrm{mm}$. La longueur du frustule varie de $11 / 4$ à $31 / 2$ c. de $\mathrm{mm}$. (Fig. 130, 2.)

Le $C$. dirupta, Greg, est une jolie espèce marine, finement ponctuée, dont le nodule médian, à la valve inférieure, est étalé transversalement en espace blanc ou stauros, qui fait une croix sur Ie raphé.

Mais l'espèce marine la plus commune est le $C$. scutellum. C'est le géant du genre, car sa longueur peut aller de $41 / 2$ à $6 \mathrm{c}$. de $\mathrm{mm}$. Il est, en outre, très reconnaissable aux stries de sa valve supérieure, transversales, disposées comme les parallêles d'une carte 
géographique et formées de gros points. Ces points sont carrés ct s'alignent en files longitudiuales parallèles, de sorte que la valve parait raye longitudinalement par les interstries. Chacune des stries transversales se termine à la zone marginale par un élargissement triangulaire, couvert de très fincs ponctuations ( $T$ à 8 strics dans 1 c. de mm.)

La valyc inferieure présente des strics transversales rayonnantes, formées de points beaucoup plus fins. Elle cst bordée, comme dans le C. placentula, d'unc zone forméc de deux lignes claires concentriques, entre lesquelles est une rangée de grosses ponctuations comme les perles de certains cadres.
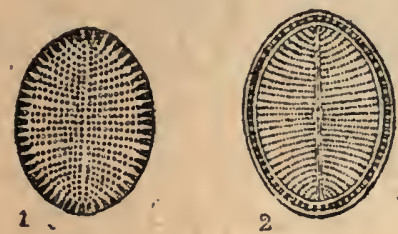

Fig. 117. - Cocconeis scutellum, Ehb. 1 , valve supérieure; 2 , valve inférieure.

Les auteurs signalent encore quelques autres espèces ou variétés toutes exotiques, parmi lesquelles nous citerons le $C$. salina, variété du $C$. pediculus, dont les lignes flexueuses longitudinales sont moins visibles, et le $C$. helvetica, très petite cspèce, trouvée par M. J. Brun dans les lacs suisses, présentant une bordure formée par une double ligne marginale sur la valve inférieure, et deux,ordres de stries, les unes atteignant le nodule central, les autres alternées avec les premières et de moitié plus courtcs. Cctto petite espèce ne mesure que 1 à $2 \mathrm{c}$. de mm. - Citons cncore le $C$. costata, Grun: jolie espèce californienne. (Fig. 130,3 ct 4.)

Achnanthes. - Les Achnanthes se distinguent facilement des Cocconeis en ce que les frustules sont plus nettement courbés en genou, quand on les regarde par la face connective, avec les bords dorsal et ventral généralement parallèles. Les valves, dissemblables, sont plus allongées, naviculoïdes, quelquefois étranglées en semelle ou cn violon au niveau du petit axe. Les autres caractères pour la disposition de l'endochròme, le raphé et les nodules sur la valve inféricure concavée, sont les mèmes que dans toute la tribu, mais les nodules tcrminaux sont moins visibles que dans les Cocconeis

Lcs Achnanthes vivent solitaires, ou réunis par deux ou un petit nombre dc frustules; ils sont portés par un pédicelle hyalin fixé à 
l'un des angles du premier frustule. (Fig. 120, 4.) Plusieurs sont marins, d'autres se trouvent dans les eaux douces ou saumàtres.

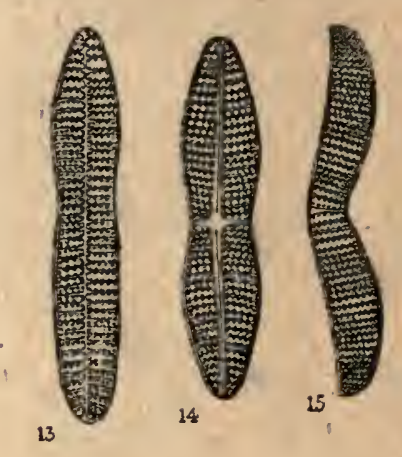

Fig. 118. - Achnanthes longipes, C. Ag.

13 , valve supérieure; 14,15 , valve inférieure

Les valves sont striées de stries furmées de points, nettement transversales ou un peu rayonnantes autour du nodule médian.

Une des plus belles espèees, marine, l'A. longipes, C. Ag., se distingue tout de suite des autres en ce que ses valres sont marquées de côtes transversales entre lesquelles sont deux rangées de points. Le nodule médian de la valve inférieure est dilaté transversalement en un espaee lisse ou stauros et le pseudo-raphé de la valve supérieure est droit. Le frustule, allongé, navieuloïde, environ cinq à six fois plus long que large, est étranglé au niveau du petit axe. La zone connective est finement strice en travers avec quelques plis longitudinaux. Cette espèee mesure de $\dddot{a}$ à $18 \mathrm{c}$. de $\mathrm{mm}$.

Les autres espèees présentent des stries transversales ponctuées, mais pas de eòtes.

Tel est l'Achnanthes brevipes, C. Ag., autre grande espèce marine, dont les valves sont plus larges, environ quatre fois plus longues que larges, rétréeies au niveau du petit axe. Le' pseudoraphé de la valve supérieure n'est pas droit, mais eourbe, et le nodule médian de la valve inférieure est dilaté transversalement; la zone connective est comme dans l'espèce précédente. Les stries, au nombre d'environ 7 dans $1 \mathrm{c}$. de $\mathrm{mm}$, sont formées d'assez grosses ponetuations, dont on compte de 2 à 10 dans chaque strie, suivant sa position sur le frustule. Cette espèee mesure de 7 à 10 cent. de millim. 
L'Achnanthes subsessilis, Ehb., diffëre du précédent par ses extrémités plus arrondies, sa forme (une ellipse allongée sans constriction au niveau du petit axe), ses stries qui sont plus fines et sa taille plus petite. Il ne mesure que de 3 à 5 cent. de millim. Il est marin.

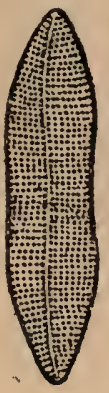

1

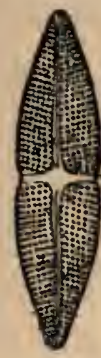

2

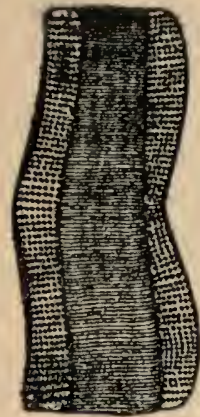

3

Fig. 119. - Achniantes brevipes, C. Ag.

1, valv. sup.; 2 , valve inf.; 3 , face connective.

L'A. parcula, Ktz, marin aussi, est encore plus petit $\left(\begin{array}{llll}1 & \text { a } & 1 & 1 / 2\end{array}\right.$ cent. de millim.) et ses stries, ponctuées, sont plus fines.Le pseudoraphé est courbe comme dans les précédents et le nodule dilaté en stauros transversal.

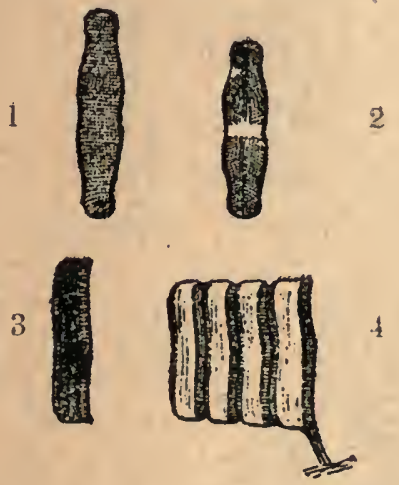

Fig. 120. - Aclinanthes coarctula, Brèts.

1 , valve sup.; 2 , valve inf.; 3 , frustule entier, face connect. ;

4, groupe de frustules pédicelles

Nous citerons encore, parmi ces espèces à stauros, les suivantes qui habitent les eaux douces:

L'A. coarctata, Bréb : valves resserrées en violon au petit axe. 
légèrement dilatées aux extrémités (capitées), avec un pseudo-raphé très excentrique et des stries ponctuées finez. Long. : 1 à $4 \mathrm{c}$. de $\mathrm{mm}$.

Les Achnanthes hungarica, Grun., et A. affinis, Grun., ont un pseudo-raphé droit et ne sont pas resserrés au petit axe; le raplié.du premier est bordé d'une zone hyaline, qui forme une croix très apparente avee le nodule central dilaté transversalement. Les stries sont fines, rayonnantes autour du nodule ( 21 dans un $1 \mathrm{c}$. de $\mathrm{mm})$. Le second est plus allongé, ordinairement plus petit de taille, $\grave{a}$ stries non rayonnantes et très fines ( 27 à 30 dans 1 cent. de mm.)

Les espèces suivantes, qui toutes habitent les eaux douees, n'ont pas le nodule médian dilaté en stauros transversal et les deux valves sont semblables quant aux stries, ne différant que par la présence du raphé et des nodules sur la valve infèrieure. Aucune n'est rétrécie au niveau du petit axe.

L'A. biasolettiana, Grun., a une forme non plus resserrée au petit axe, mais dilatée. Il est très petit (environ 1 cent de millim.) avec des stries un peu rayonnantes autour du nodule et fines : 22 à 28 dans 1 e. de $\mathrm{mm}$.

L'A. delicatuia, Grun. est relativement plus renflé encore au petit ase et ses extrémités sont pointues (subrostrées). De plus, ses stries sont grosses ( 1 da dans $1 \mathrm{c}$. de $\mathrm{mm}$.), et son nodule médian est entouré d'un petit eerele hyalin. Il est un peu plus grand que l'espèce préeédente.

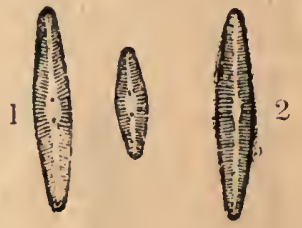

Fig. 121. -- Aclinanthes exilis, Kz.

1 , valves inférieures; 2 , valve supérieure.

L'A. microcephela, $\mathrm{K} \%$, est très long, presque bacillaire, avec les extrémités capitées et des stries très fines (30 à 36 dans 1. c. de m.); l'A. exilis, Kz, a les extrémités arrondies, mais non eapitées (26 à 27 stries); l'A. minutissima a les extrémités un peu rostrées, et l'A. linearis, W. Sm., qui lui ressemble beaucoup, est plus arrondi par les bouts et relativement moins long. L'un et l'autre ont de 24 à 27 stries dans. 1 c. de mm., mais l'A. minutissima est près de einq fois aussi long que large, tandis que l'A. linearis n'est qu'environ quatre fois aussi long que large.

Toutes ces espèces sont fort petites, mesurant de 1 à 2 cent. de mm., sauf l'A. exilis, qui peut atteindre $3 \mathrm{c}$. 
Enfin l'A. lanceolata, Brèb., petite espèce cncore, se distingue tout de suite, non seulement par sa forme en ellipse courte et scs grosses stries (env. 1 ij dans 1 c. de mm.), mais encore par sa valve supérieure, qui présente au nivcau du petit axe, sur un seul de ses côtés, un espace lisse en forme de fer à cheval.

Plusieurs auteurs, Kützing, Grunow, H. Van Heurck, etc., ont séparé des Achnanthes proprement dits, sous le nom d'Achnanthidium, des cspc̀ees ou variétés dont le raphé, au lieu d'ètre droit ou simplement courbe, est sigmoïde, c'est-à-dire tourné en S. Tel est l'Achnanthes flexella, Bréb., ou Achnanthidium flexellum, assez grande espècc d'eau douee ( 4 à 3 cent. de millim.), à stries rayonnantes, fines et délicatement ponctuées.

L'Achnanthidium flexellum, avee son raphé sigmoïde, et l'Achnanthes lanceolata avec sa marque hyalinc qui interrompt les stries sur l'un des còtés de la valve inférieure, semblent établir le passage aux Cyclophora, classés par Grunow au nombre des Cocconeis et dont M. Castracane a fait un genre, adopté par M. P. Petit. Ce genre Cyclophora, dont nous ne connaissons qu'une espèce, exotique, a une forme elliptiquc en écusson, comme les Cocconeis, un raphé et un pseudo-raphé sigmoïdes, comme les Achnanthidium, et sur l'un des còtés des deux valves, une figure lisse comme en présente sur une seule de ses valves l'Achnanthes lanceolata. Cette figure est un petit cercle entouré d'une minee bordure, comme une moulure, avee un gros grain au centre, le tout imitant un bouton de sonnette.

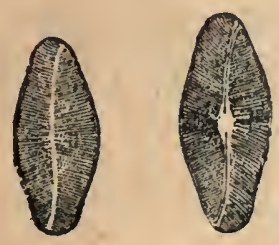

Fig. 122. - Achnanthidium flexellum, Bréb.

Ajoutons que les valves de ce Cyclophora d'Australic sont perlées, que la valve inférieure ne présente ce bouton de sonnette que sur l'un des còtés du nodule médian, et que la valve supéricure porte de chaque côté de son large pseudo-raphé sigmoïde deux surfaces claires en forme de croissant allongé à coneavité regardant le pseudo-raphé. C'est sur l'une de ces surfaces que parait appliqué le bouton de sonnette de cette valve. 
Les GoMphoxÉnÉEs ont un cndochròme formé d'unc seule lame qui repose par son milicu sur un des còtés de la zone connective.

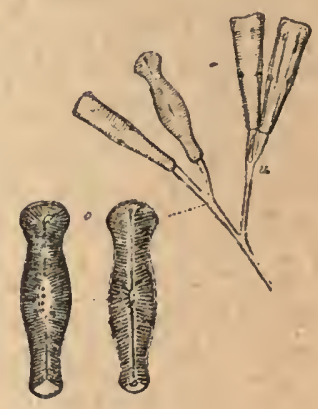

Fig. 123. - Gomphonema Geminatum, Ag.

s'étend de chaque côté de celle-ci de manière à doubler les deux valves jusque vers le milieu de l'autre còté de la zone où ses deux bords se rejoignent.

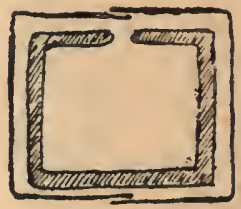

Fig. 124. - Coupe schématique d'un Gomphonema, d'après Borscow.

Les frustules; dans cette famille, sont en forme de coin, qu'on les regarde par la face valvaire ou par la face connective, mais surtout par cette dernière. Les valves, très souvent cunéiformcs, cn effct, ne le sont pas toujour's régulièrement et présentent souvent des dilatations et des constrictions transversales, et quelquefois mème ont une forme naviculoïde plus ou moins allongée; mais elles ont toujours une extrémité plus élargie quel'autre, qui est plus pointue. Ces valves ont un raphé et sont symétriques par rapport à lui. Elles ont deux nodules terminaux ct un nodule central, qui n'occupe ordinairement pas le milieu du raphé, mais est un peu plus élcvé vers l'extrémité la plus dilatée de la valve. Elles sont striées trans- 
versalement de stries en points. L'extrémité la plus mince présente souvent une petite surface claire.

Les Gomphonémées ont les frustules portés sur un coussinet ou thalame hyalin, ou bien sont fixés par leur extrémité atténuée à l'extrémité d'un pédicelle. Ils se détachent d'ailleurs facilement et nagent librement dans l'eau. (Voir PI. II, fig. כ้ et 7 .)

Cette tribu ne comprend que deux genres:

$1^{\circ}$ Rhoïcosphenia, Grun.; $2^{\circ}$ Gomphonema, Ag.

Rhoïscophenia. - Ce genre établit le passage de cette famille à la précédente par ses frustules qui, bien que cunéiformes, sont courbés latéralement en genou comme chez les Achnanthes, et n'ont, comme ceux-ci, de véritable raphé et de nodules que sur la valve concave ou inféricure, la valve supérieure n'ayant qu'un pseudoraphé.

Le Rhoïcosphenia curvata, Grun. est une assez petite espèce, longue de $11 / 2$ à 4 cent. de mm., qui vit dans les eaux douces; on en trouve cependant unc variẻté marine d'une taille ordinairement un peu plus grande. Les valves, vues à plat, ont une forme naviculoïde, mais avec une extrémité un peu plus pointue que l'autre. La valve inférieure présente un cercle hyalin autour du nodule central et des stries un peu rayonnantes, qui ne vont pas tout à fait jusquau

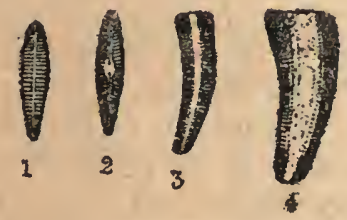

Fig. 125. - Rinoïcosphenia curvata, Gr.

1 , face valvaire supérieure; 2 , face valv. inf.; 3,4 , face connective.

raphé (env. 12 dans 1 cent. de $\mathrm{mm}$.). La valve supérieure a des stries un peu plus grosses, allant jusqu'au pseudo-raphé, et un point clair a chaque extrémité. La membrane connective est lisse, bordée de deux lignes légères qui se terminent à chaque bout par une encoche.

Gomphonemá. - Les Gomphonema se distinguent facilement des Rhoïcosphenia en ce que leurs frustules, vus par Ia face connective, ne sont point arqués, mais cunéiformes droits, symétrigues 
par rapport au grand axe; ils présentent, d'ailleurs, les caractères généraux de la famille, chaque valve ayant un nodule médian, un peu remonté vers l'extrémité la plus large, et deux nodules terminaux. Les stries, qui sont ponetuées, rayonnent autour du nodule qu'entoure souvent un ecrele hyalin ou stauros, et les valves seraient eomplètement symétriques si, dans presque toutes les espèees, on ne voyait sur l'un des côtés de la valve un ou quelquefois plusieurs gros points isolés, près du nodule médian. Ils sont d'ailleurs portés dans leur jeune àge sur un thalame mueilagineux ou sur un pédieelle diehotomique. (Vuir Fig. 123 et PI. II, Fig. .̌, 6, 7.) Les espèees sont asse\% nombreuses, vivent dans l'eau de mer ou dans les eaux douces, ct fournissent un grand nombre de variétés.

On peut arriver assez aisément à distinguer ces espèces en opérant diverses coupes dans la tribu. Ainsi, deur espèces sculement, parmi eelles de nos pays, ne présentent pas de point asymétrique sur le còté du nodule médian. L'une est le Gomphonema olivaceum, Kz, commun dans nos eaux douces, et l'autre, le G. exiguum, $\mathrm{Kz}$, qui est marin.

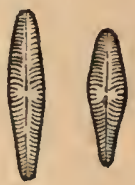

Fig. 126. - Gomplionema olivaceum, Kz.

Le Gomphonema olivaceum a une forme navieulö̈de, mais plus atténuéc à l'une de ses extrémités.

Le nodule médian, remonté vers l'extrémité la plus large, est entouré d'un espaee lisse et le raphé bordé d'une minee bande hyaline. Les stries sont légèrement rayonnantes autour du nodule médian, au nombre de 10 dans 1 cent. de $\mathrm{mm}$. L'extrémité atténuée de la valve montre une petite surface claire. Le frustule ne dépasse pas $3 \quad 1 / 2$ eent. de $\mathrm{mm}$. Cette espèee fournit une variété souvent mêlée à l'espèce type, G. olivaceum vulgare, un peu plus petite et plus large.

Le Gomphonema exiguum, Kz. ressemble beaueoup au précédent comme forme et comme taille, mais il est marin et plus allongé. Ses stries sont plus serrées (18 dans 1 eent. de millim.) presque parallèles, laissant ainsi une mince zone hyaline le long du raphé, Mais eette zone s'élargit peu autour du nodule.

Les autres espèces présentent un ou quelquefois plusieurs gros points isolés à eòté du nodule médian; mais les unes ont un frustule présentant des dilatations et des constrietions quand on les regarde 
par la face valvaire, d'autres ont une forme naviculoide ou lancéolée.

Parmi les espèces dont les ralves présentent une ou deux constrictions, nous signalerons les suivantes :

Gomphonema constrictum, Ehb. Vu par la face valvaire, le frustule a la forme d'une téte de marteau, le nodule central représentant le trou du manche.

Les valves sont en effet très dilatées au nireau du petit axe, puis resserrées et de nouveau dilatées à l'une des extrémités, qui est capitée. C'est une jolic espèce, assez commune dans nos eaux douces à cours rapide, remarquable par ses stries rayonnantes autour du nodule et qui, alternativement, atteignent et n'atteignent

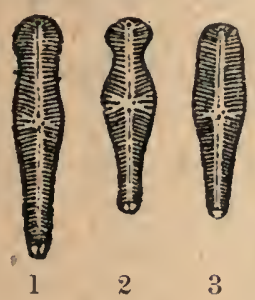

Fig. 127. - 1. Gomphonema constrictum var. subcapitatum, Ehb; 2. Gomphonema constrictum, Ehb. ; 3. G. capitatum, Ehb.

pas le nodule ; c'est cette disposition qui produit l'apparence d'un pseudo-stauros. Le raphé est, dù reste, bordé d'une zone hyaline. L'extrémité atténuéc du frustule montre un espace clair au delà du nodule terminal. Les stries sont fortes, finement ponctuées; il y en a 10 à 12 dans 1 cent. de mill. et le frustule mesure de 4 à 6 centièmes de mill.

Le G. capitatum, Ehb., est considéré comme une variété du précédent. Il n'en diffère que parce que les valves sont moins étranglées; c'est-à-dire que, malgré son nom, il est moins capité que l'autre.

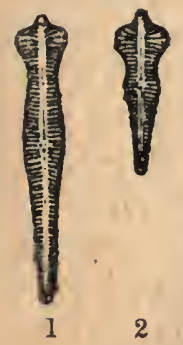

Fig. 128. 1, Gomph. acuminatum, Ehb.; 2, G. acum. laticeps, Ehb. 
Gomphonoma acuminatum, Ehb. Il a la mème forme générale en marteau, mais l'extrémité large est benucoup plus élargie que le milieu de la valve au petit axe. De plus, elle se termine en pointe (rostrée).

La bordure hyaline le long du raphé est très marquée, mais très peu dilatée autour du nodule médian, sur le eòté duquel on voit un point isolé. La strie eorrespondant à ce point de l'autre eòté de la valve est écourtée. Les stries, finenent ponctuées, sont un peu rayonnantes et au nombre de 10 à 12 dans un cent. de mill. Sa longueur est de 1 1/2 à ä c. de mm.

Cette espèce, assez eommune dans les ruisseaux et les nares, fournit un grand nombre de variétés, qui ne diffèrent guère que par leur forme plus ou moins large ou longue. Tels sont les Gomphonema acuminatum laticeps, Ehb., G. acum. coronatum, P. Petit, G. acum. elongatum, P. Petit, etc.

Gomphonema augur, Ehb. Il est beaueoup plus large que le préeédent, mais ses valves n'offrent pas de eonstrietion; il est en forme de eerf-rolant avee une pointe mousse sur son extrémité large. C'est aussi une espèee d'eau douee, dont les stries ressemblent à eelles du $G$. acuminatum, et sa taille ne dépasse pas s cent. de mill.

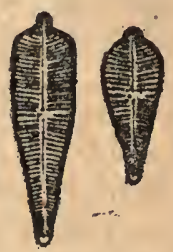

Fig. 129, - Gomphonemá augur, Ehb.

Gomphonema geminatum, Ag. Il est ainsi nommé paree que ses frustules se reneontrent le plus souvent groupés deux par deux aux extrémités de son pédieelle ramifié. Ces frustules sont renflés au petit axe, resserrés au-dessus et au-dessous, et dilatés de nouveau aux deux extrémités, mais beaucoup plus à l'une de ees extrémités qu'à l'autre. Sur la large surface lisse qui entoure le nodule médian, on voit, d'un côté, de 5 à 8 petits grains isolés. Les stries sont fortes, ponctuées, rayonnantes ; on en compte de 9 a 10 dans 1 eent. de mill. (J. Brun) et le frustule mesure de 8 à 12 eent. de mill. C'est une des plus belles espèces de cette tribu. (Fig. 123.)

Gomphonema micropus, Kz. Il a une forme générale navieuloïde, mais avec une extrémité un peu plus élargie que l'autre, la partie médiane dilatée avec une très légère eonstriction au-dessus et au-dessous. L'aire hyaline qui longe le raphé est notablement 
élargie en stauros autour du nodule médian; il n'y a qu'un seul point latéral isolé. Les stries sont écourtées à ce niveau et un peu rayonnantes. Il y a 10 stries dans 1 cent. de mill. Le frustule a environ 3 cent. de mill. de longueur. Eaux douces. (Fig. 130, 8.)

Les espèces suivantes présentent souvent une dilatation plus ou moins marquée du frustule au niveau du petit axe, mais elles n'offrent plus de constrictions proprement dites; elles vont en

2

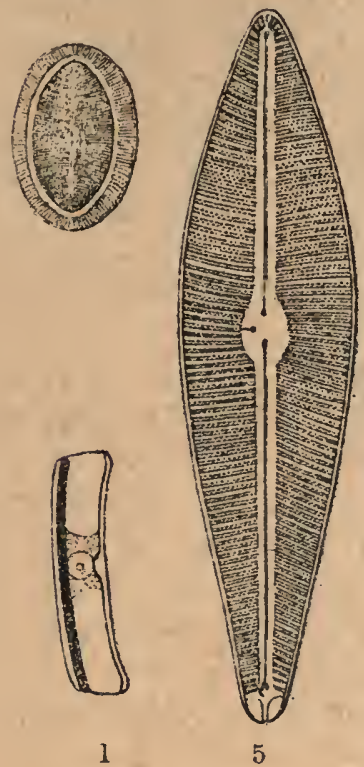

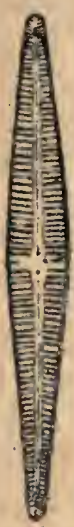

6
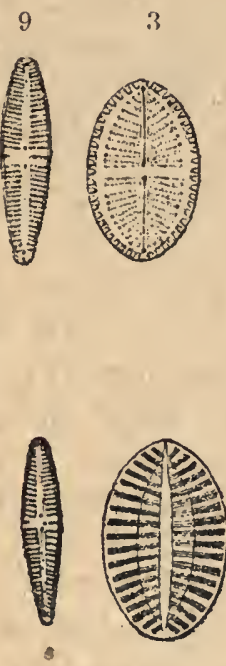

8

Fig. 130. - 1, Disposition de l'endochrôme et du plasma dans un Cocconeis, d'après Borscow;

2, Cocconeis placentula, Ehb. valve inf.;

3,4 , Cocc. costata, valve inf. et sup.;

5, Gomphonema mamille, Ehb.;

6, Gomph. gracile, Ehb.;

7, Gomph. Ventricosum, var. ornctum; .

8, Gomph. micropus, Kz.;

9, Gomph. ungustatum, Grun. (Gross. 600 diam.)

s'atténuant progressivement vers les extrémités, l'une de cèlles-ci toujour's moins atténuée que l'autre. L'aire hyaline est assez large autour du nodule médian. Ce sont des espèces d'eau douce.

Le Gomphonema tenellum, $\mathbf{K z}$, ressemble beaucoup au G. micropus, et l'un peut ètre regardé comme une variété do l'autre. Les constrictions au-dessus et au-dessous du petit axe dilaté sont encore moins marquées. Les valves sont un peu plus 
larges et moins longues et l'espèce est plus petite. Les frustules ont 15 stries dans 1 cent. de mill. (J. Brun) et sont portés sur un pédicelle très court.

Gomphonema parvulum, Kz. C'est une espèce assez commune, a valve lancéolée, cunéiforme. assez large, avec une petite pointe mousse sur l'extrémité large (rostrée). Il y a 14 stries dans 1 cent. de mill. et le frustule mesure ęnviron 3 centièmes.

Gomphonema gracile, Ehb. Cette espèce a une forme longue nettement naviculöde, les ralves allant en s'atténuant régulièrement au-dessus et au-dessous du petit axe élargi ; les deux extrémités sont presque sémblables, et ce n'est guère que théoriquement que les valres sont cunéiformes. Elles sont réellement en losange très allongé. La bande hyaline le long du raphé est extrêmement mince, mais elle se dilate beaucoup autour du nodule médian, où elle présente un point latéral isolé. On compte 9 à 10 stries dans 1 cent. de mill. et le frustule peut atteindre jusqu'à près de 10 centièmes. (Fig. 130,6.)

Le Gomphonema dichotomum, W. Sm., est classé par M. Van Heurck comme une variété du G. gracile. Il présente, en effet, des caractères analogues, sauf que les bords des valves sont un peu plus courbes et les extrémités un peu plus obtuses. Le frustule est moitié plus petit èt les stries plus fixes (12 à 13 dans 1 c. de mill.)

Le Gomphonema intricatum, $\mathbf{K z}$, est naviculoïde lancéolé, long; le frustule est dilațé à la partie médiane et de là va en s'amincissant presque autant vers les deux extrémités qui finissent par une courbe presque aussi obtuse. La raphé est bordé d'une bande hyaline assez large, notablement élargie au nodule. 8 à 10 stries dans 1 cent. de $\mathrm{mm}$. Le frustule a de 4 à 6 centièmes.

Gomphonema angustatum, Kz. C'est peut-être une variété du précédent, plus large, malgré son nom. Il est presque régulièrement naviculoïde ou quelquefois avec une constriction à peine sensible au-dessous de ses extrémités. Les autres caractères sont les mèmes que chez le précédent, mais les stries sont un peu plus serrées (10 à 11 dans 1 centième). Le frustule est ordinairement un peu plus petit. (Fig. 130,9.)

Cette tribu des Gomphonemées contient encore plusieurs espèces et variétés, dont on trouvera la figure dans les Atlas spéciaux (1), et qui sont presque tous exotiques. Nous représentons ci-contre (Fig. 130, 5), le beau Gomphonema mamilla, Ehb., de Fall River dans l'Orégon.

(1) Voir H. Van Heurck, Atlas de la Synopsis des Diat. de Belg. 


\section{$3^{\epsilon}$ T RIBU}

\section{CYMBELLEES}

Les Cymbellées ont un endochròme formé d'une seule lame reposant par son milieu sur la zone connective, tantòt du còté le plus concave tantôt du côté le plus convexe, suivant les espèces, puis doublant les deux valves adjacentes et se rejoignant sous la zone opposée. En effet, les frustules sont dyssymétriques, renflés d'un còté de manière à présenter un dos (còté convexe) et un ventre (côté

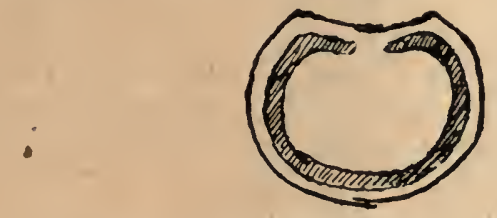

Fig. 131. - Schéma de la disposition de l'endochrôme dans un Cymbella ; coupe d'après Pfitzer.

concavé). Les valves sont gonflées à leur centre, et, comme on dit, cymbiformes ou cintrées; elles ne sont pas symétriques par rapport à leur grand axe, mais seulement par rapport au petit axe. Elles présentent un raphé, courbe ou droit, un nodule médian et deux nodules terminaux.

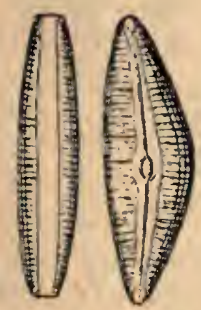

Fig. 132. - Cymbellä Ehrenbergii, Kz.

1 , face valvaire; 2 , face connective.

Beaucoup d'espèces de cette tribu sont, à l'état jeune, portées sur un pédicelle simple ou ramifié, ou bien contenues dans des tubes gélatineux. Plus tard, le plus grand nombre des frustules s'affranchissent de leur support ou de leur enveloppe, et, ainsi libérées, certaines espèces deviennent parfois assez difficiles à distinguer les unes des autres. 
II. P. Petit fait rentrer dans les Cymbellées les cinq genres suivants :

$1^{\circ}$ Cocconema, Ehb. - 2० Encyonema, Kz. - $3^{\circ}$ Amphora, Ehb. - $4^{\circ}$ Epithemia, Bréb. - Cymbella, Ag.

En raison des grandes analogies que présentent plusieur's espèces de ces divers genres, certains auteurs ont, comme M. H. Van Heurck, réuni les Cocconema et les Cymbella dans le seul genre Cymbella, et M. J. Brun y a mème joint des espèces du genre Encyonema.
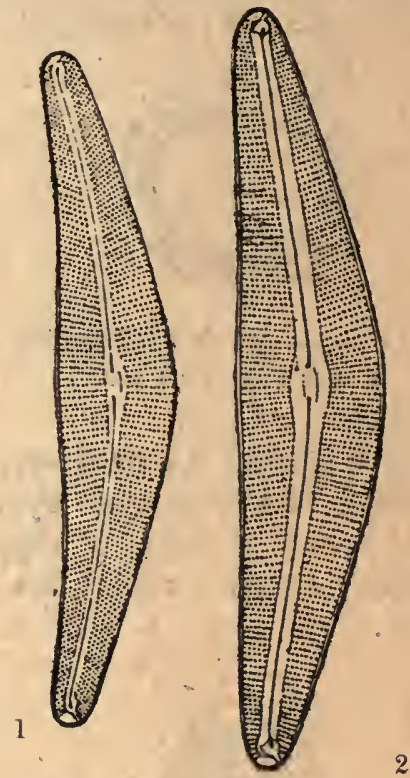

Fig. 133.-1, Cocconema lanceolatum, Ehb. ; 2, Cocçonema gaströ̈des, $\mathrm{Kz}$

Cocconema. - Le genre Cocconema, Ehb., contient des espèces à frustule long, peu large, fortement cintré, de sorte crue le dos est très convexe et le ventre nettement concave, et ordinairement un peu renflé à la partie médiane, à la hauteur du nodule. Le raphé est courbe, bordé d'une zone hyaline plus ou moins large, plus ou moins dilatée autour du nòdule médian. Les valves sont striées de stries perlées, en général robustes et légèrement rayonnantes autour du nodule médian et du raphé.

Les frustules jeunes sont portés seuls ou par paire sur un pédicelle filamenteux simple ou dichotome, ce qui rappelle les Gomphonema. Ils forment ainsi par leur réunion des couches mucilagineuses sur les objets submergés, et se séparent plus tard pour nager librement. 
Nous pouvons donner pour type de ce genre le Cocconema lanceolatum, belle espèce très répandue dans nos eaux douces, présentant d'une manière très nette tous les caractères typiques de ce genre. Le bord dorsal est très convexe, le raphé très arqué, la zone hyaline assez étroite, le nodule médian très grand et allongé. Les stries sont au nombre de sept à huit dans 1 cent. de mm. et la longueur du frustule est de 8 à 1 s̆ cent. de mm.

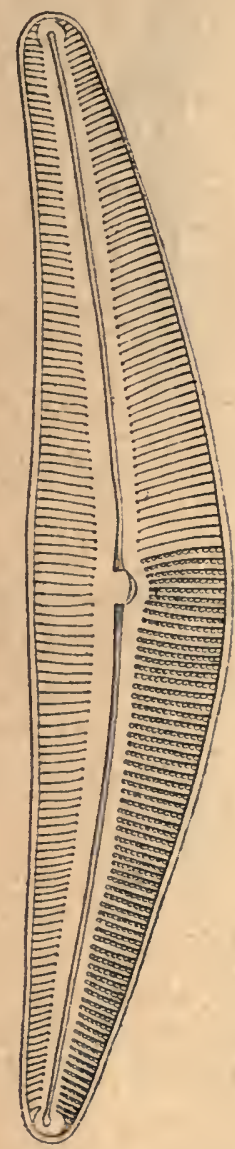

Fig. 134. - C. asperum, Ehb, d'après M. P. Petit.

Le Cocconema asperum est une espèce très voisine, mais qui se distingue de la précédente par la lảrgeur de la bande hyaline le long du raphé. D'après M. Paul Petit, elle atteint 22 c. de $\mathrm{mm}$. de longueur sur 4 de largeur et présente 8 stries dans 1 c. de mm. (1).

(1) P. Petit et Lenduger-Fortmorex. Des Gisements siliceux fossiles de l'Auvergne. (Journ. de Micrographie T. II, 1887.) 
Le Cocconema sistula, Ehb, sur lequel M. Paul Petit a étudié le phénomène du rajeunissement des cellules diatomées. (Voir p. 48 et Pl. I.) est ausši une des espèces sur lesquelles M. Matteo Lanzi pense avoir observé la sporulation ou la formation de germes. (Voir p. 50 et Pl. II, Fig. 3 et 4.) Il est relativement moins long et plus large que les précédents. Il est très arqué aussi, mais le ventre est.

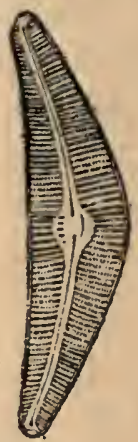

Fig. 135.- Cocconemá cistula, Ehb.

moins concave, et la zone hyaline le long du raphé est, relativement aussi, moins large et plus dilatèe autour du nodulemédian. Sur cette partie hyaline, près du nodule médian et du côté ventral, on voit de 2 à $ّ$ granules isolés, ce qui rappelle encore certains Gomphonema. Il est plus petit de taille, variant de 5 à 9 c. de $\mathrm{mm}$. et ne présente que 7 à 8 stries ponctuées dans 1 cent. de $\mathrm{mm}$. Il est commun dans les eaux douces.

Le Cocconema cymbiforme, Ehb, est mince et long, mais très peu arqué; aussi le raphé est-il très peu courbe, et partage-t-il la valve en deux parties presque égales. La bande hyaline qui le borde est étroite, à peine dilatée au nodule médian, avec un grain isolé du côté ventral. La valve est légêrement bombée au centre. Il y a de 8 à 11 stries (J. Brun) dans 1 cent de mm. et le frustule a de 5 à 10 cent. de mill.

Cette espèce, assez commune dans les eaux douces, fournit plusieurs variétés, dont une, plus petite de moitié, $C$. cymbiforme parvum, W. Sm., est assez répandue.

Le Cocconema gastroïdes, $\mathrm{Kz}$, est encore une grande espèce ressemblant assez au $C$. lanceolatum, mais ayant le côté ventral beaucoup moins concave, presque droit, un peu renflé au centre. Le raphé est par conséquent assez peu arqué, mais la bande hyaline qui le borde est très large, peu dilatée autour du nodule. Les stries sont 
robustes, 8 dans 1 c. de $\mathrm{mm}$. Le frustule peut atteindre jusqu'à 13 cent. de $\mathrm{mm}$.

Quant au Cocconema tumidum, Bréb, large et court, avec le dos extrèmement bombé tandis que le ventre est presque plat et que les deux extrémités se détachent comme les deux bouts d'un chapeau bicorne, il nous paraic faire le passage des Cocconema aux Encyonema, bien qu'il soit pédicellé. La bande hyaline du raphé est large, dilatée au nodule et présente quclquefois un ou deux granules isolés. Le nodule médian est ordinairement traversé par un sillon. Il y a 8 à 9 stries robustes dans $1 \mathrm{c}$. de $\mathrm{mm}$. et le frustule mesure environ 6 cent. de $\mathrm{mm}$.

Encyonema. - Ce genre est remarquable par la forme du thalle ou thalame, qui contient les jeunes frustules. C'est un tube mucilagineux, hyalin, dans l'intérieur duquel les frustules sont renfermés et où ils se multiplient par division, se poussant les uns les autres en avant et dans des positions alternativement inverses.

Les frustules sont ordinairement plus trapus que ceux des Cocconema, avec un dos généralement très convexé tandis que le ventre est presque droit, sauf que les extrémités s'infléchissent du còté du ventre. Le raphé est droit et partagc la valve en deux parties très inégales; le nodule médian est grand et les nodules terminaux, éloignés des extrémités, sc prolongent dans l'axe, oblique, de ces extrémités. Les stries sont ponctuées et rayonnantes.

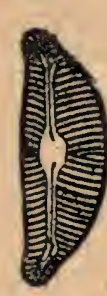

$a$
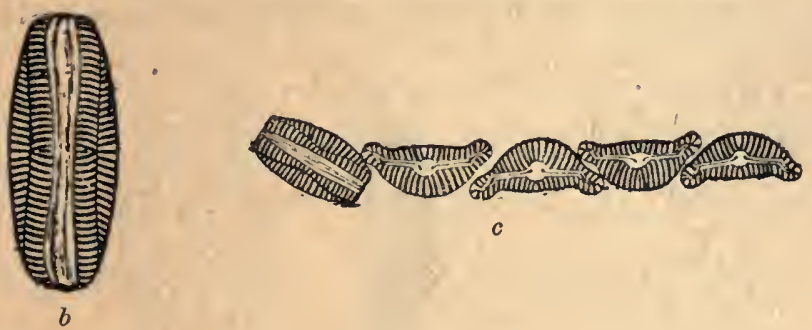

Fig. 136 - Encyonema prostratum, Ralfs.

$a$, face valvaire, $b$, face connective $; c$, frustules contenus dans leur tube mucilagineux.

L'Encyonema prostratum, Ralfs, peut être pris pour type. C'est une belle espèce, sur laquellc on voit bien les caractères. Les nodules terminaux se prolongent longuement dans l'axe des extrémités bien détachécs et inclinées du còté ventral. L'aire hyaline autour du nodule central est large et certaines stries, à ce nivcau, sont plus courtes que les autres, formant comme les plis autour d'un ombilic. Vu par la facc connective, lc frustule est large, très brus- 
quement tronqué aux deux extrémités. Les stries sont très fortes, perlées, 7 à 8 dans 1 cent. de millim. Cette espèce, d'eau douce. habite des tubes toujours simples et mesure de 6 à 9 cent. de mill. de longueur.

L'Encyonema cospitorum, Kz, est plus court, plus trapu, a le dos plus bombé, les extrémités moins arrondies et moins inclinées vers le ventre. L'aire hyaline centrale est petite; les stries sont plus fines (8 à 12 dans 1 c. de mm.) et le frustule est moitié plus petit. Il habite dans des tubes ramifiés. (Eaux douces.)

LEncyonema ventricosum, Kz, est un peu plus allongé, le bord ventral est droit, les extrémités presque pointues ne se recourbent

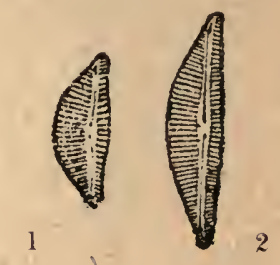

Fig. 13\%. - 1, Encyonema cespitosum, Kz.;2, Encyonema centricosum, Kz.

pas vers le ventre et restent dans l'axe da raphé. La bande hyaline le long du raphé est très étroite, non dilatée autour du nodule médian. Les stries sont faibles, serrées (12 à 16 dans $1 \mathrm{c}$. de mm.) et le frustule est fort petit, ne dépassant guère 2 c. 1/2 de nım.

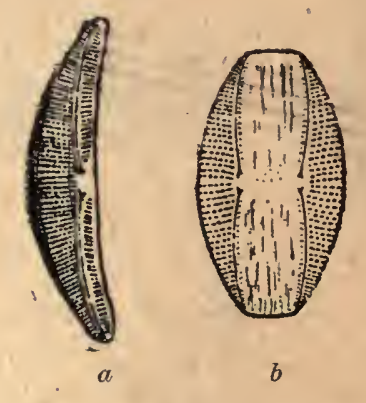

Fig. 138. - Amphora ovalis, $\mathrm{Kz}$

$a$, face valvaire; $b$, face connective.

Amphora. - Ce genre se distingue par la forme extrêmement dyssymétrique de ses valves. C'est ainsi que, vus par leur face valvaire, les frustules présentent un côté dorsal plus ou moins bombé ou convexe, avec un còté ventral peu concave ou même tout à fait droit; mais le raphé est très excentrique et situé tout près du bord 
rentral, quelquefois mème presque tout à fait sur le bord, de sorte que le nodule médian peut ètre marginal. Ce nodule médian est quelquefois dilaté en stauros. Les nodules terminaux sont situés tout à l'extrémité des valves. Vus par la faee conneetive, les frustules ont une forme généralement ovoïde, et la zone eonnective est souvent striéc, plissée ou ponetuée longitudinalement.

Nous pouvons prendre pour type l'Amphora ovalis, Kz, dont le eòté ventral est un peu eoneave, et dont le raphé, courbé dans le mème sens, et à double courbure, partage le petit axe en deux partics très inégales qui sont entre elles environ comme 1 : 2. Le nodule médian, presque marginal, ne se dilate pas en stauros. Les stries, très légèrement rayonnantes, soht robustes, à grosses ponetuations, au nombre de 10 à 11 dans un eent. de mill. Cette espèce, d'eau douce, commune partout, a de $\ddot{b}$ à 7 cent. de mm. de longueur.

Elle fournit plusieurs variétés, dont l'une, A. ovalis gracilis, Ehb., est plus étroite et plus grèle. La variété $A$. ovalis affinis, $\mathbf{K z}$., qui se trouve aussi dans les eaux un peu saumàtres, est aussi plus petite, avec les extrémités plus pointues et les stries plus fines (12 à 13 dans 1 cent. de mm.).

L'Amphora minutissima, IV. Sm. est une très petite espèce d'eau douce, qui vit en parasite sur d'autres Diatomées, par exemple sur les Nitzschia linearis et $N$. sigmö̈dea (J. Brun). Elle est très renflée et les frustules vus par la face conneetive sont presque ronds. Le nodule médian est dilaté en un espace lisse qui coupe presque la valve dans toute sa largeur sur le petit axe. Les stries sont, très fines, 30 dans 1 cent. de mm. (J. Brun). Sa taille n'est que de $1 / 2$ à $21 / 2$ centièmes de millimètre.

L'Amphora lineolata, Ehb., a un frustule plus àllongè, en ellipse longue par la face eonnective, des stries très fines (23 dans 1 cent. de $\mathrm{mm}$.) et une zone eonnective marquée aussi de stries transversales très fines, coupées par de nombreux plis longitudinaux. La longueur est de 4 à ö eent. de $\mathrm{mm}$.

La plupart des autres espèces sont marines. Parmi les plus remarquables nous eiterons les survantes :

Amphora ocellata. Donk. dont les frustules, vus par la face eonnective, n'ont pas un contour ovale, mais quadrangulaire. Le nodule médian est prolongé en un stauros qui coupe la valve suivant le petit axe. Les stries sont très fines. Le frustule est grand et peut atteindre 9 eent. de $\mathrm{mm}$.

Amphora lavissima, Greg. Cette espèec ressemble à la précédente quant au nodule médian prolongé en stauros transversal, 
mais le frustule est long et minec. Les stries sont extrêmement fines.

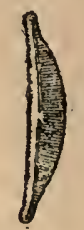

Fig. 139. - Amphora salina, W. Sm.

Amphora salina, W. Sm. Espèce longue, à raphé marginal, sans dilatation transversale du nodule médian. Les cxtrémités, légèrement renflées s'incurvent un peu du còté du ventre. Les stries sont fines, 18 à 21 dans 1 cent de mm. La taille est de 3 à $\breve{~ c e n t . ~}$ de $\mathrm{mm}$.

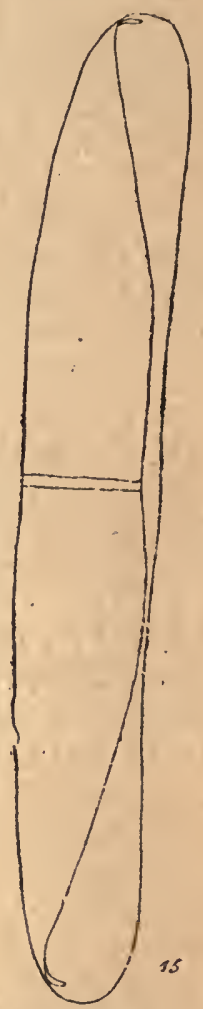

Fig. 140. - Amphora cingulata, $\mathrm{Cl}$.

L'Amphora cingulata, Cl. est une grande espèce marine, des mers des Indes et des Antilles, dont le stauros est étendu transver- 
salement, en ceinture. Elle a environ 1.3 stries dans 1 cent. de $\mathrm{mm}$. et mesure de 8 à 10 cent. de $\mathrm{mm}$. (La lïg. 140 n'en représente que le contour à un grossissement de 800 diamòtres.)

Epithemia. - Ce genre a été placé par le prof. Pfitzer et par M. Paul Petit dans les Crybelcées, parce que son endochróme présente la mème disposition que dans les autres genres de cette tribu, c'est-à-dire qu'il est formé d'une seule lame reposant par son milieu sur la zone connective convexe ou dorsale (comme dans les Amphora), s'étendant sous les deux valves adjacentes et allant se joindre sous la zone connective cencave ou ventrale, où se trouve la ligne de suture. Les frustules sont, elr effet, gonflés d'un còté du petit axe, comme ceux des genres que nous avons décrits précédemment, présentant un dos plus ou moins convexe et un ventre concave. Mais ils se distinguent par l'absence de vèritable raphé et des nodules médian et terrninaux.

C'est en raison de l'alssence de véritable raphé que M. I. L. Smith, M. H. Van Heurck et tous les auteurs qui ont adopté le système de classilication fondé sur la présence ou l'absence d'un raphé, ont rangé les Epithemia dans une classe très éloignée des CyMbelúses, (qui, ayant un raphé et des nodules, appartiennent par conséquent au groupe des Raphidées), et les ont placés dans le groupe des Pseudo-Raphidées, qui n'ont qu'un faux raphé et pas de nodules.

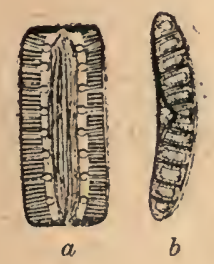

Fig. $141-$ Epithemia Argus, Kz. $a$, face valvaire; $b$, face connective

Il est évident que, non seulement à cause de la disposition de leur endochròme, mais encore en raison de leur forme et de leur aspect général, les Epithemia se rapprochent beaucoup des Amphora. Nous avons vu, eu cffet, que si l'on part des Cocconema, en passant par les Encyonema et les Amphora, on trouve des frustules arqués avec un còté bombé, le còté opposé plus ou moins rentrant, et un raphé qui divise la valve en deux parties de plus en plus dissemblables, la partie dorsale devenant toujours de plus en plus grande, tandis que la partie ventrale devient de plus en plus petite; si bien que nous avous fini par trouver des Amphora chez lesquels 
le raphé longe presque le bord ventral de la valve et ou le nodule médian est porté tout à fait sur la marge.

Si l'on suppose que la migration du raphé vers le bord ventral continue au delà de sa position dans les Amphora, on peut admettre qu'il finit par sortir de la valve et disparaitre. On a alors un frustule arqué, strié en travers et qui n'a plus de raphé ni de nodule. C'est un Epithemia.

D'autre part, si le raphé véritable a disparu, chez beaucoup d'espèces on voit apparaitre une ligne longitudinale, un pseudo-raphé, droit ou infléchi, qui aboutit souvent à une sorte de nodule médian.

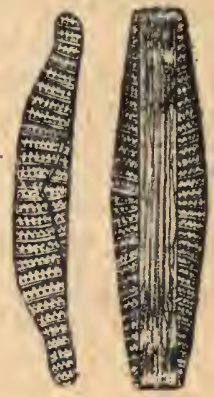

Fig. 142

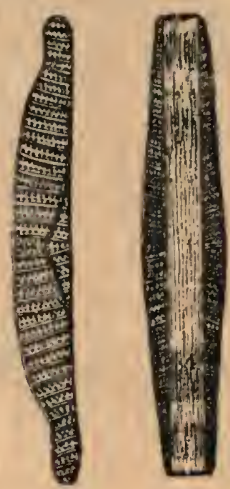

Fig. 143

Fig. 142. - Epithemic turgida, Ehb.

Fig. 143. - Epithemia turgida, var. granulata, Grun.

Enfin ajoutons, comme détail particulier, que les Epithemia présentent des épaississements internes, visibles à l'extérieur sous l'aspect de cotes plus ou moins robustes. Ces côtes, que W. Smith considérait comme des canalicules, ont une direction à peu près transversale sur la valve et sont souvent dilatées aux extrémités, c'est-à-dire au bord des valves, où elles forment par leur coupe optique comme une rangée de grosses perles quand on regarde le frustule par la face connective.

Entre ces còtes, on voit sur les valves des stries perlées, à peu près transversales et plus ou moins serrées.

Vus par la face connective, les frustules sont souvent gonflés en leur milieu, aux extrémités du petit axe.

Les Epithemia ne sont point portés sur des stipes ni dans des tubes, mais ils vivent ordinairement accolés par le còté concave de leur zone connective sur d'autres plantes aquatiques, qu'ils 
recouvrent d'une couche mueilagineuse. Les frustules sont le plus souvent isolés un par un, quelqucfois géminés, et forment rarement des séries plus nombreuses.

L'Epithemia turgida, Ehb., est une des espèces les plus belles et les plus communes sur les plantes aquatiques dans nos eaux douces. Le frustule est long et assez mince, et la valve est environ six fois plus longue que large, bien que la forme de eette espèce soit très variable. Le bord dorsal est assez fortement convexe, mais le bord ventral est presque droit, sauf aux extrémités, qui sont atténuées, un peu capitées et s'infléchissent du côté ventral. Les còtes transversales sont robustes, celles du centre rayonnant autour du milicu du bord dorsal; il y en a de quatre à six dans 1 ecnt. de mill., et entre elles est une rangée de grosses perles oblongues composées chacune de deux petites perles rapprochées. Ces perles s'alignent aussi en séries longitudinales, ou plutòt diagonales, en écharpe. Il y a environ huit de ces séries perlées dans la largeur de la valve. Du bord ventral se détache un pseudoraphé formé de deux lignes courbes venant se couper au centre de la valve sur une demi-côte impaire partant du bord dorsal et qui marque le petit axe. Au-dessus et au-dessous de ce petit axe, les deux demi-valves sont rigoureusement symétriques.

La face connective a une section rectangulaire très allongée, un peu r'enflée au milieu.

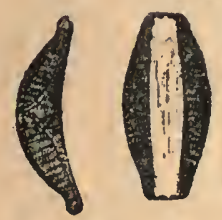

Fig. 144. - Epithemia sorex, Kz॰ (d'après le Dr Van Heurck.)

Cette belle Diatomée peut avoir de $\mathbf{7}$ à $10 ّ$ cent. de millimètre de largeur.

On pense que l'E pithemia Hyndmanni, W. Sm., qui ressemble extrèmement à l' $E$. turgida, mais qui est beaucoup plus grand et atteint 20 cent. de mill., n'est que son frustule sporangial.

L'Epithemia sorex, $\mathrm{Kz}$, est moitié plus petit, plus trapu, plus arqué, avec un bord ventral très concave, des cxtrémités atténuées capitées, qui ne s'infléchissent pas du côté du ventre, mais plutòt du côté du dos. Vu par la face valvaire, le frustule a tout à fait la forme d'un petit chapeau bicorne. Les deux lignes courbes qui représentent le pseudo-raphé se prolongent jusqu'au bord dorsal 
où elles se coupent, embrassaut dans leur angle d’intersection un espace clair imitant un pseudo-nodule. Les còtes sont moins robustes que dans l'E. turgirle; il y en a 6 à 7 dans 1 cent. de

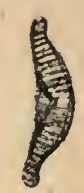

Fig. 145.

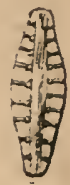

Fig. Ifli.

Fig. 145. - Evithemia sores, Kz. id'apres M. 1. Truan .

Fig. 146. - Epithemia orellata, Ehb. 'f. conn.)

mill., et elles comprennent aussi den langs de perles très tin es. Vu par la face connective, le frustule est benucoup plus épais cl plus bombé au centre que dans l'E. turgida.

C'est une espèce d'eau douce, assez communc et qui mesure de 2 a 4 cent. de millim. - On connait sa forme sporangiale dont le frustule est très analogue, quoique relativement moins larçe. mais dont la longueur est presque doulıle.

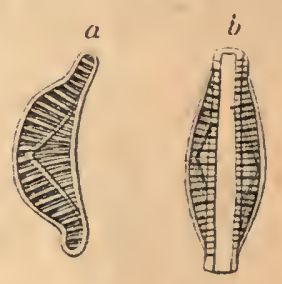

Fig. 147. - Epithemıa gibberula, Ehb. (d'après M. Paul Petil).

L'Epithemia gibberula, Ehlb. ne nous parait ètre qu'une variété de l'E. sorex.

L'Epithemia gibba, K\%. cst encore une espèce d'eau douce très répandue. Elle a un frustule très long et très mince, presque bacillaire, avec des valves très étroites, dont le bord veniral est droit et le bord dorsal presque droit aussi, sauf une bosse au milieu du dos. L.es deux extrémités, pointues, se recourbent en crochet du cóté du ventre. Il n'y a pas de pseudo-raphé formé par deux lignes courbes qui se coupent an centre, mais une seule ligne longitudinale yui longe le bord dorsal. Les còtes sont fortes, 6 a 7 dans 1 cent. de mill., à peu près parallòles, sauf aux extrémités. Elles comprennent deux rangées de stries linement perlées. Le frustule peut atteindre $20 \%$ cent. de mill. 
L'Epithemia gibba fournit quelques variétés, dont l'E. gibbn. ventricosa, Gr., qui est plus petit, plus trapu et plus bossu. (Voir

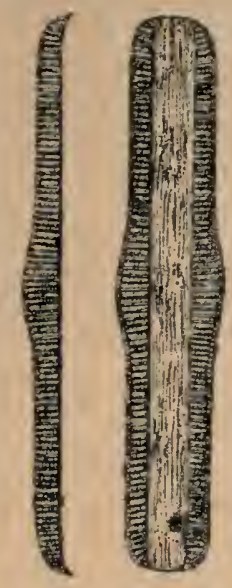

Fig. 148 .

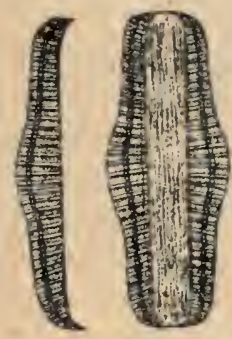

Fig. 149.

Fig. 148. -- Epithemia gibba, Kr.

Face connective et face valvaire

Fig. 149. - Epithemia gibba ventricosa, Gr.

PI. II. Fig. 1, 2); et l'E. gibba parallela, Gr., qui est, au contraire, plus long, plus mince et n'est pour ainsi dire plus bossu du tout sur le dos.

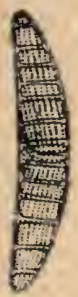

Fig. 150. - Epithemiu sebra, К\%.

L'Epithemirs sebia, Kz., est une julie espece, plus petite, tris peu arquée, presque cylindrique, à extrémités arrondies, non recourbies, zébrées en travers de cótes presque parallèles ( 3 à $31 / 2$ dans 1 cent. de-mill.) comprenant entre elles, non plus deux, mais quatre ou cinç stries perlées. On voit apparaitre, près du milieu du bord ventral. deux très courtes lignes courbes qui se coupent tout de stuite près de ce bord et représnntent un psendo-raphé très 
abrégé. Vu par la face connective, le frustule donne une coupe optique en rectangle allongé, sans renflement. C'est encore une espece d'eau douce, mais qu'on trouve ausși dans les eaux saumàtres et dont la taille varie de 2 à 6 cent. de mill.

L'Epithemia Argus, Kz, ressemble au précédent, mais il est un peu plus grand, moins arqué encore, avec des extrémités arrondies, comme un cigare qui serait un peu courbé. Les côtes sont très robustes, presque parallèles, écartées ( 1 à 2 dans 1 cent. de mm.) contenant entre elles 6 ou 7 stries finement perlées. Le pseudo-raphé règne sur toute la longueur de la valve, formé de deux lignes qui, venant des extrémités, longent le bord ventral et vont par une courbe abrupte se couper sur le bord dorsal. - Vu par la face connective, le frustule montre de chaque còté de la zone une rangée de grosses perles, ou ocelles produits par la coupe optique des còtes, très dilatées à leurs extrémités.

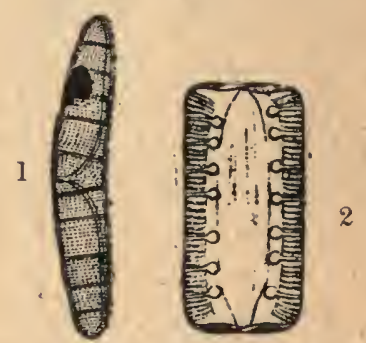

Fig. 151 .

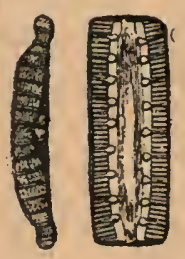

Fig. 152 .

Fig. 151 - Epithemia Argus, Kz.

1 , Frustule vu par la face valvaire; 2 , autre frustule vu par la face connective

Fig. 152 - Epithemia Argus, var. amphicephala, Grun.

Cette espèce, d'eau douce, a de 4 à 7 cent. de mm. de longueur. Elle fournit.des variétés à bords ondulés ou à extrémités capitées, fort élégantes, telles que l'Ep. Argus amphicephala, Grun.

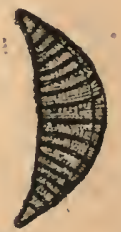

Fig. 153 - Epithemia musculus, Kz:

Ce genre renferme aussi quelques espèces marines, parmi lesquelles nous citerons le joli Epithemia musculus, dont la face 
valvaire est en demi-lune avec des còtes rayonnantes et un pseudoraphé formé d'une ligne qui longe le bord dorsal et montre un petit nodule médian. La face connective est ovale. Les stries sont finement perlées, et le frustule mesure de 4 à ò cent de millim.

Cymbella. - Le genre Cymbella, $\mathbf{A g}$, aux dépens duquel ont été formés les genres Cocconema et Encyonema, tend au retour à la symétrie des valves par rapport aux deux axes. Il forme ainsi le passage des Cymbellées, asymétriques, aux Naviculées, symétriques.

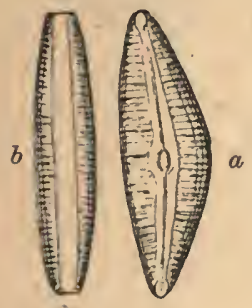

Fig. 15i - Cymbella Ehrenbergii, Kz.

$a$, Face valvaire; $b$, Face connective.

Ainsi, les Cocconema comprennent des espèces à frustules arqués, à dos convexe et à ventre concave, de sorte que le bord dorsal et le bord ventral sont plus ou moins parallèles; dans les Encyonema, le bord ventral se redresse, il est à peu près droit, sauf aux extrémités, qui se recourbent souvent de manière à lui conserver une courbe générale concave. En mème temps, le raphé se rapproche de plus en plus du bord ventral et les valves sont de moins en moins symétriques par rapport à ce raphé. Dans les Epithemia, le bord dorsal est toujours convexe, le bord ventral tantôt concave, tantôt presque droit et le raphé a disparu tout à fait, parfois remplacé par un pseudo-raphé de forme diverse. Chez les Cymbella, le bord dorsal reste convexe; mais le bord dorsal non seulement n'est plus concave, non seulement n'est plus droit, mais il devient convexe à son tour, de sorte que les deux bords latéraux sont courbés en sens contraire, étant tous deux convexes, mais le dos plus que le ventre, ce qui conscrve au frustule une forme encore un peu arquée. Il en résulte que le raphé reparait et tend à prendre la position médiane qu'il occupe normalement dans les frustules réguliers, et, par exemple, dans les Naviculées.

Nous pouvons donc donner comme caractères de ce genre la forme lancéoléc plus ou moins arquée du frustule, avec le bord dorsal et le bord ventral tous deux convexes, des valves asymé- 
triques divisées en deux parties inégales par un raphé plus ou moins courbe, présentant des nodules terminaux et un nodule médian excentrique. Pas de fausses cloisons, ni de còtes, mais seulement des stries perlées transversales, souvent plus serrées aux extrémités que dans la partie médiane. Les frustules, à l'état jeune, sont

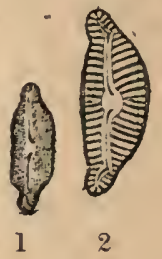

Fig. 155. - 1, Cymbella anglica. Lag.'; 2, Encyonema prostratum, K\%.

fixés, solitaires ou par paires, ou groupés, dans une masse gélatineuse hyaline formant comme un sac, qui sc prolonge quelquefois en pédicelles portant un ou deux frustules (comme nous l'avons décrit pour le Gomphonema olivaceum, d'après M. Matteo Lanzi, Pl. II, Fig. 7). Ils s'en séparent avec l'àge, et e'est à l'état libre qu'on les trouve le plus souvent.

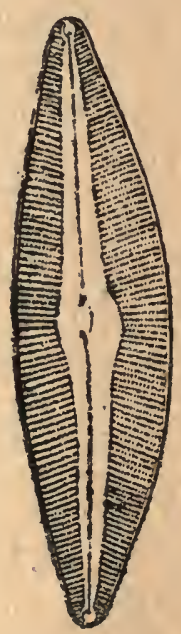

Fig. 156. - Cymbella Elirenbergii, Kz.

Nous n'arons à signaler que des espèees d'eau douce. Le démembrement dont ce genre a été l'oljet n'y a, d'ailleur's, laissé qu'un assez petit nombre d'espèces, sujettes, il est vrai, à diverses variations. Il faut avouer, du reste, une certains Encyonemn pour- 
raient aussi bien ètre rangès parmi les C'ymbella, s'ils n'étaient dans leur jeune àge contenus dans des tubes hyalins à forme bien définic. (Fig. 19\%.)

Nous donnerons comme type la plus belle et la plus grande espece, le Cymbella Ehrenbergii, Kz, dont le frustule a des valves largement lancéolées, avec des extrémités un peu atténuées el rostrées, un raphé notablement rapproché du bord ventral, bordé d'une large zone hyaline très peu dilatée autuur du nodule médian. Les stries sont fortes (environ 8 dans 1 cent de $\mathrm{mm}$ ), mais très finement perlées. La longueur du frustule varie de 6 i 13 cent. de mill.

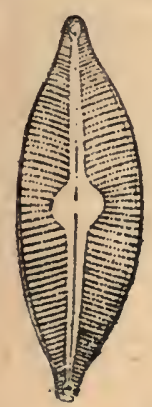

Fig. 157. - Cymbella cuspidata, Kz.

Le Cymbella cuspidata, Kz., se reconnait tout de suite à ses extrémités pointues. La zone hyaline qui borde le raphé, courbe, est étroite, mais très dilatée au nodule médian. Les stries sont grosses ( 6 environ dans 1 cent. de $\mathrm{mm}$.) mais deux fois plus serrées aux extrémités, finement divisées. Sa longueur ne dépasse pas 8 cent. de $\mathrm{mm}$.

Toutes les autres espèces sont de beaucoup plus petite taille. Ce I'est pas là un caractère bien scientifique, mais il permet néanmoins de les distingner à première vue des deux précédentes.

Le Cymbella obtusa, Greg. a une furme naviculée, à bord ventral presque droit, un raphé un peu flexueux bordé d'une étroite bande hyaline, faiblement dilatée au nodule médian. Les stries sont au nombre de 12 dans 1 cent. de nm. au milieu, 13 aux extrémités. Le frustule ne dépasse pas 3 cent. de $\mathrm{mm}$.

Le Cymbella affinis, $\mathrm{Kz}$. , a les frustules courts, trapus, à bord dor'sal très arqué, à bord ventral un peu convexe, avec un raphé très arqué, parallèle au bord dorsal, bordé d'une zone hyaline étroite. Les extrémités sont rostrées; les stries médianes sont au nombre de 9 dans 1 cent. de $\mathrm{mm}$. du côté du dos, de 11 du côté du ventre. 
C'est une petite espèce d'eau douce et qui ne dépasse guère $21 / 2$ cent. de millim.
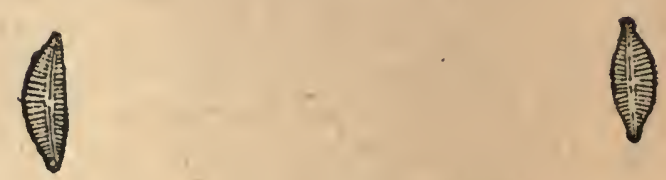

Fig. 158.'-Cymbella affinis, Kz. Fig. 159.-Cymbella amphicephala, Næg.

Le Cymbella leptoceras, $\mathrm{Kz}$, a les extrémités atténuées, le dos très arqué, le ventre gonflé aree un raphé non pas courbe, mais un peu flexueux, bordé d'une large zone hyaline. Environ 8 stries dans 1 eent. de $\mathrm{mm}$. Espèce d'eau douce, longue de 2 à $3 \mathrm{c}$. de mm.

Signalons encore le Cymbella stomatophora, Gr., espèce exotique signalée par M. P. Petit aux environs de Paris, et qui se distingue de toutes les précédentes par la présencc d'un ou deux points isolés dans l'aire hyaline près du nodule central, du côté le moins courbe de la valve, et par un léger étranglement au-dessous des extrémités des valves. Il y, a 8 à 9 stries dans 1 eent de $\mathrm{mm}$. et le frustule a 7 à 8 cent. de $\mathrm{mm}$. de long.

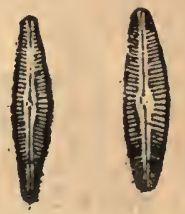

Fig. $160 \quad$ Fig. 161

Fig. 160. -Cymbella gracilis, Kz. Fig. 161. - Cymbella subaequalis. Grun.

Les Cymbella amphicephala, Neg, et C. gracilis, Kz, sont encore deux petites espèces de 1 1/2 à 4 c. de mill., dont la première est remarquable par ses extrémités capitulées, et la seconde par sa forme grêle.

Le Cymbella.subaqualis, Gr., a une forme naviculoïde avec un raphé presque droit, qui partage la valve en deux parties presque égales. C'est une forme de passage des Cymbella aux Navicula. Les stries sont un peu plus serrées que dans le précédent, 10 dans 1 cent. de mm. au milieu et 14 aux extrémités. Petite espèce d'eau douee, de 2 à 3 eent. de $\mathrm{mm}$. 


\title{
X \\ DESCRIPTION DES PRINGIPALES ESPĖCES
}

\author{
$4^{\mathrm{me}}$ TRIBU \\ NAVICULËES \\ $\S 1$. - Caractères
}

La tribu des NAVICULÉES présente ce caractère remarquable que l'endochròmeest formé de deux lames séparées, dont chacune repose par son milieu sur la zone connective et se relève plus ou moins sur les valves adjacentes jusqu'au voisinage du raphé. (Fig. 162, 3.)

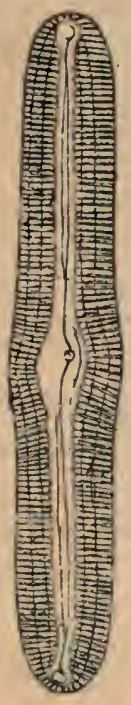

1
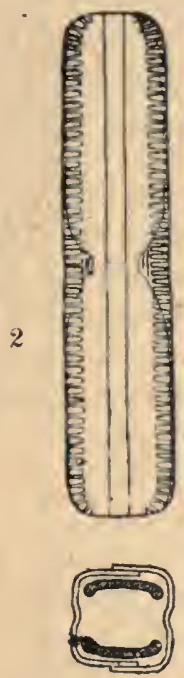

3

Fig. 162. - Navicula major, Kz.

1 , face valvaire. 2 , face connective.

3 , Coupe transversale montrant la disposition de l'endochrôme.

Cette tribu, la plus nombreuse de toute la famille des Diatomées, comprend des espèces dont les frustules ont des valves presque toujours symétriques par rapport au grand axe, et le plus souvent même par rapport aux deux axes. Le grand axe, marqué par un 
raphé, peut ètre droit ou sigmoïde; il présente toujours un nodule médian et deux norlıles terminaux. Il y a toujours des stries, disposées quelquefois en systèmes compliqués, le plus sourent perlées. mais parfois rémnies en còtes plus ou moins robustes.

Les frustules ont une forme régulière, naviculaire ou fusiforme, (eu navette, en nacelle on en fuscau), elliptique plus ou moins allongée, quelquefois resserréc au milicu, en guitare, ou présentant des dilatations et des constrictions successives, mais symétriques des deux côtés. Quelquefois enfin, le frustule, plıs ou moins fusiforme, est sigmoïde, c'est-ì-dire tordu en S.

Quelques espèces ont, dans le jeune àge, les frustules renfermés dans des tubes mucilagineux hyalins, d'antres dans des masses gélatinenses amorphes; d'autres encore ne sont recourerts que d'une couche de coléoderme, pour ainsi dire individuelle. Dans tous les eas, la plupart paraissent se débarrasser de très bonne heure de leur thalame pour nager librement dans l'eau ambiante, et e'est parmi les Naviculées ru'on trouve les espéces douées des mouvements les plus rapides et les plus persistants.

Cette tribu, avons-nous dit, est la plus nombreuse de tontes; elle renferme une longue liste de genres, presque tous très chargés d'espèces, et d'autant plus chargés que les diatomistes, cherchant avec raison la simplification, ont supprimé, dans ees dernières années, certaines coupes génériques jui n’étaient peut-être pas toutes bien nettement earactérisées, mais dont quelques-unes, au moins, facilitaient la détermination des espèces.

M. Paul Petit admet les 14 genres suivants :

Navicula, Bory.

Schizonema, Ag.

Brebissonia, Grun.

Mastogloia, Ag.

Stigmaphora, Wall.

Stictodesmis, Grun.

Diadesmis, Kr.
Stauroneis, Ehb.

Scoliopleura, Gr.

Pleurosigma, W. Sm.

Donkinia, Ralfs.

Toxonidea, Donk.

Berkeleya, Ehb.

Amphiplenira. Kz.

La tribu des Cymbeluées se rattache à celle des Naviculées par le genre Cymbella, dont certaines espèces, comme le Cymbella subaqualis, ont les frustules presque droits et les valres presque symétriques par rapport à leur grand axe, et se rapprochent beaucoup de certains Navicula. D'autre part, il y a dans le genre Navicula quelques espèces qui se rapprochent spécialement de ecrtains Cymbella, par exemple le Navicula spharophora, dont l'endochrôme est disposé comme celui des Cymbella et dont les valves ne sont pas tout à fait symétriques par rapport au grand axe, 
rn laison de l'absence des stries sur l'un des còtés du nodule central.

D'autre part, Ics Naviculées, par les genres Donkinia et Toxonidea, se rapprochent de certains Epithemia, avec lesquels lcur's frustules ont d'éridentes analogies d'aspect.

Nous ne pouvons ici, comme on le comprend, qu'indiquer les principales espèces de cette très nombreuse tribu, et parmi cclles-ci nous choisissons de préférence celles qu'on rencontre en Europe, et particulièrement en France, en Belgique, en Suisse, en Allemagne, en Italie ou en Espagne.

\section{ล. 2. - Genre Navicula.}

Le grand genre Naricula comprend des espèces dont les fiustules ont une forme cn nacelle ou en navettc, naviculaire, - car e'est

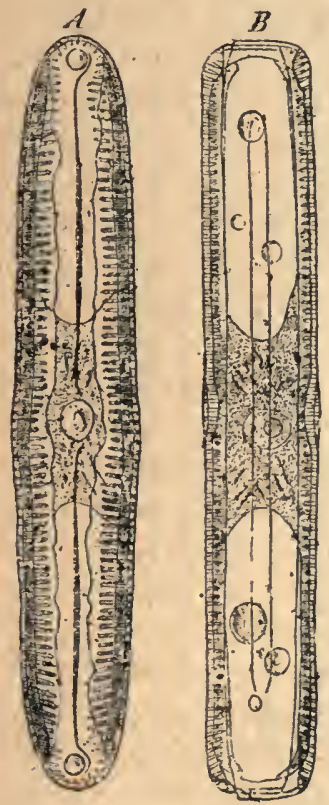

Fig. 163. - Navicula viridis, Kz.

Montrant la disposition des yalves, l'endochrôme, le plasma et le noyau.

$A$, face valvaire; $B$, face connective,

coupe longitudinale faite suivant le raphé, montrant la cyclose des glubules huileux (d'après E. Strasburger)

de cettc forme mème que la tribu a pris son nom, - ordinairement beaucoup plus longue que large, quelquefois cependant assez largement ellipsoïde. Les valves présentent un raphé droit avec deux 
nodules terminaux ct un nodulc médian; clles sont symétriques par rappor't à leurs dcux axes, et sont maryuées de côtes plus ou moins robustes, parfois lisses, e'est-à-dire non résolubles en perles, mais le plus souvent divisécs en travers ou composécs de grains ou perles séparables au microscope.

La facc connective du frustule a ordinaircment une forme bacillaire plus ou moins allongée, montrant sur ses bords l'extrémité des stries ct au milieu, de chaque còté, l'encoche produite par le nodule médian. (Fig. 162 et 163.) Elle peut présenter des dilatations ou des étranglcments cn différentes parties.

Les espèces de ce genre sont ordinairement libres et douées de mouvements très actifs.

Pour nous guider dans la description de ces espèces, nous şommes obligés de pratiquer parmi elles des coupes, souvent artificielles, qui nous aidcront à les classer.

C'est ainsi que nous séparerons d'abord toutes les espèces dont les valves présentent les strics transversalcs robustes que nous avons appelées côtes, et qui nc sont point divisibles en scgments ni résolubles en perles ou grains séparćs. Ces côtes paraissent, en elfet, réellement lisses, étant formées par la soudure des grains qui doivent résulter de la structure aréolaire commune, à ce qu'il semble, aux valves de toutes les Diatomées, ainsi que nous l'avons expliqué antérieurement. (Voir p. 7\%.) Quelquefois, cependant, ces côtes ne sont pas absolument lisses, et présentent des divisions, très peu marquées il est vrai, mais encore résolubles, indiquant que si les grains aréolaires sont soudés, ils ne le sont pas d'une manière complète et peuvent encore ètre discernés. Néanmoins, mème dans ce cas, il s'agit encore d'une còte montrant des divisions légères et non une strie perlée, c'est-à-dire formée d'une succession de grains nettement séparés les uns des autres.

Les espèces qui présentent ce caractère formaient l'ancien genre Pinnularia d'Ehrenberg, genre que l'on n'a peut-ètre pas eu raison de raycr des classifications méthodiques. Elles ont toutes une forme longue avec les extrémités arrondies, forme en bàton très caractérisée, et une physionomie três spéciale. Elles ne forment plus maintenant, dans le genre Navicula, que le sous-genre Pinnularia ou le groupe des Navicules Pinnulariées.

Elles se distinguent tout de suite, par lcurs côtes, de toutes les autres espèces, qui sont munies de stries divisibles ou perlées, plus ou moins fines, plus ou moins parallèles ou rayonnantes et qui constituent les autres groupes des NA viculÉEs.

Examinons d'abord les Pinnulariées.

Nous avons dit qu'elles ont une forme longue, en bàton, mais elles peuvent ètre plus ou moins gonflées aux deux extrémités ou au 
milieu, sur la face valvaire; quelquefois mème le bord des valves présente deux ou trois ondulations ou festons. Le raphé, quoique droit dans sa direction générale, peut éprouver quelques flexuosités.

On les divise en grandes et petites espèces ou, comme on dit, en majeures et mineures. Les majeures peuvent atteindre jusqu'à 40

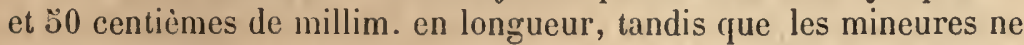
dépassent guère 10 à 13 cent. et encore pour un très petit nombre d'espèces, car la plupart n'ont que de 4 à 7 centièmes.

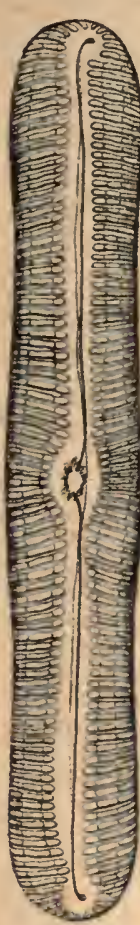

Fig. 161

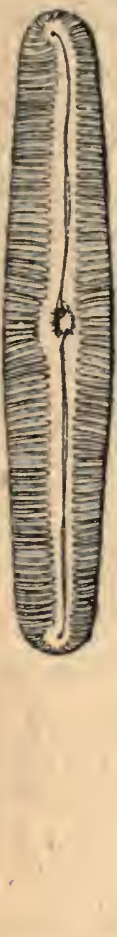

Fig. 165

Fig. 164. - Navicula (Pinnularia) nobilis, Ehb. Fig. 165. - Navicula (Pinn). major, Kz.

Cette distinction empirique est d'ailleurs très défectueuse, car mème les grandes espèces, comme le $N$. viridis, peuvent présenter de très petits individus, n'ayant par exemple que $ّ$ centièmes de millimètre de longueur.

Quoi qu'il en soit, le groupe des Pinnulariées majeures renferme quatre des plus belleś espèces d'eau douce, et quelques-unes marines un peu plus petites.

Le Navicula (Pinnularia) nobilis, Ehb, est une magnifique 
Diatomée, en bàton, légèrement dilatée au milieu, sur la face valvaire, et très légèrement gonflée aux deux bouts. Les còtes, très grosses, ne vont pas jusqu'au raphé; il y en a "j à 6 dans 1 cent. de $\mathrm{mm}$.

Cette espèce, d'eau douce, qui a de 20 à 40 cent. de millim. de long, donne une variété encore plus grande, le Naricula (Pinnularia) dactylus, moins gonflé encore au milieu et aux extrémités, a còtes encore plus grosses, et qui peut atteindre 30 centièmes de $\mathrm{mm}$. de long, e'est-i-dire un demi-millimètre.

Le Navicula (Pinnularia) major, Kz., autre belle espece l'eau douce, un pert plus petite (Fig. 49,162, 163̈), est plus ou moins renflée au centre, mais non aux extrémités. Les còtes, robustes, s'approchent assez près du raphé, mais laissent un large espace oblong autour du nodule médian. Il y en a de $\check{5}$ á 7 dans 1 cent. de mm. et le frustule a de 18 à 30 cent. de $\mathrm{mm}$.

Le Yavicula (Pinnularia) viridis, Kz., ne présente plusaucun gonflement; il a la forme d'une ellipse très allongée, avec des còtes au nombre de $7 \mathrm{env}$. dans $1 \mathrm{c}$. de mm., laissant un certain espace le long du raphé, mais moins autonr du nodule médian que dans l'espèce précédente. Il est plus petit, mais de taille très variable, allant de ž à 20 cent. de mm. (Eau douce.) (Fig. 163, 167). Cette espèce est une de celles où l'on réussit le miéux à voir le mouventent intracellulaire des globules d'apparence huileuse autour du noyau.

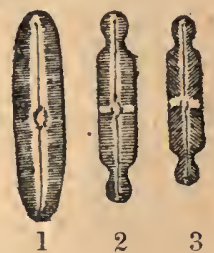

Fig. 166. - 1. Nav.(Pinnu.) viridis, var. commutata, Gr.

2. Stauroneis anceps, H.

3. Nav. (Pinn.) bicapitata, Lag. d’après A. Truan.

Sa variété $N$. (Pimn.) viridis commutata (Fig. 166, 1), Gr., a les còtes beaucoup plus fines et les deux valves ne sont pas semblables. Sur l'une, lẹ côtes laissent un espace beaucoup plus large que sur l'autre autour du nodule médian. (Eau donce.)

La dernière espèce d'eau douce que nous signalerons est le Naricula (Pinn.) cardinalis, Ehb. Elle est quclquefois un peu dilatée au centre, gonflée aux extrémités, mais souvent aussi ne présente aucun gonflement, avec des còtes au nombre de 5 env. dans 1 cent. de $\mathrm{mm}$., laissant une large zone hyaline le long du raphé sinueux et un vaste espace lisse autour du nodule médian, qui forment une 
grande croix sur la valve. Ces còtcs sont notablement rayonnantes autour du nodule médian, et tout à fait autour des nodules terminaux. Les frustules mesurent de $1 \ddot{3}$ à 20 centièmes de mm.

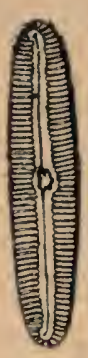

Fig. 167

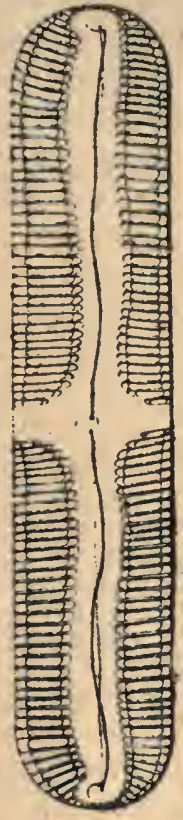

Fig. 168

Fig. 167. - Navicula (Pinn.) viridis, Kz. Fig. 168. - Nav. (Pinn.) cardinalis, Ehb.

Parmi les espèces marines, nous signalerons lc Navicula (Pinnularia) rectangulata, Greg. qui ressemble au précédent, avec les extrémités largement arrondics, et un gonflement médian; mais les còtes, beaucoup plus serrées, rayonnantes aussi autour des nodules, ne laissent qu'une étroite bande lisse le long du raphé et un petit cspace autour du nodulc médian. Vu par la face connective, le frustule est tronqué à anglc droit à ses extrémités et resserré au niveau du nodule médian.

Cette espêce ne dépasse pas $\mathbf{i}$ cent. de mm.

Lcs Naviculées Pinnulariées dites mineures ont, les unes, les bords unis, sans ondulations ni constriction au milieu; les autres ont, au contraire, les bords bi ou tri-ondulés et une constriction manifeste au niveau du nodule médian.

Parmi celles dont les bords sont unis, nous citerons les suivantes : Le Navicula (Pinnularia) latà, Breb. C'est encore une espèco 
d'assez grandc taille, et formant, pour ainsi dirc, le passage des « majeurcs » aux « mincures ". Elle a des còtes très robustes, 4 ou כ̌ dans un centième de mm,, n’atteignant pas le raphé et laissant un espace hyalin autour du nodule médian; elles sont un peu rayonnantes dans la partie médianc autour de cc nodule. Le frustule a une facc valvairc elliptique longuc, tronquée aux extrémités, une face connectivc rectangulaire à angles mousses. Cette Diatoméc vit dans les eaux douces des montagnes et mesurc de ö à 11 ccnt. de mill.

Elle présente plusicurs variétés qui la relicnt à l'espèce suivante.

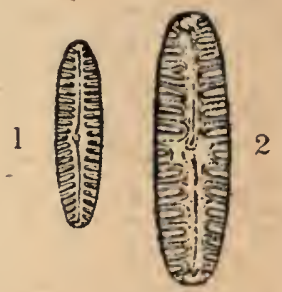

Fig. 169

Fig. 169. - 1, Navicula (Pinn.) borealis, Ehb.

Fig 2, Le même montrant les lignes de points

Le Navicula (Pinnularia) borealis, Ehb., a une forme clliptique sur la face valvaire avec des còtes assez fortcs, $ّ$ à 6 dans un cent. de mm., mais entre lesquelles on distingue des lignćs de points, plus ou moins nettement, suivant les échantillons. Les còtes, un peu rayonnantes, atteignent presque lc raphé, et laisscnt peu d'espace lisse autour du nodulc médian. Cette espèce, dont la longueur varie de 3 à 6 cent dc mill., habite les eaux douces, mais on la trouvc aussi sur les mousses qui couvrent les rochers et les murs liumides.

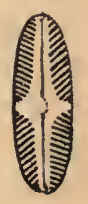

Fig. 170. - Nav. (Hinn.) Brebissonii, Kz.

Toutes les autres espèces de cc groupc ont les còtes beaucoup plus serrées, tout en nc préscntant pas de bords ondulés ni de constriction médiane. Chez certaines, les còtes laisscnt un espace hyalin trìs marqué le long du raphé et autour du nodule médian. Tcls sont : 
Le Naricula (Pinnularia) Brebissonii, Kz.; jolie Navicule d'eau donce, régulièrement elliptique, marquée de còtes rayonnantes qui manquent au milieu, laissant ainsi un large espaee hyalin autour du nodule central, espaee qui forme une croix avec celui qui borde le raphé. Il y a environ 11 còtes dans un eent. de mill. et le frustule varie de 4 à $\ddot{~}$ centièmes de mm. - Elle fournit des variétés: plus large (N.Br. subproducta), et plus grêle (N. Br.diminuta).

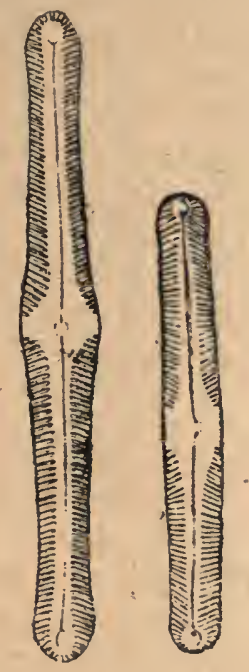

Fig. 171 Fig. 172

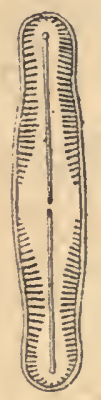

Fig. 173

Fig. 171. - N. (Pinn.) Tabellaria, var. stauroneiformis, V. H.

Fig. 172. - Navicula (Pinn.) stauroptera, Ehb).

Fig. 173. - Nav. (Pinn.) stuuroptera, Kz (d'après P. Petit).

Le Navicula (Pinnularia) stauroptera, Grun. est plus allongé, légèrement gonflé au milieu et renflé aux extrémités. Les côtes sont courtes et l'espaee lisse qu'elles laissent le long du raphé s'élargit notablement autour des nodules terminaux, beaucoup autour du nodule eentral. Elles peuvent même manquer tout à fait au niveau de ee nodule. On en compte 10 à 12 dans 1 cent. de mm. et la valve atteint 10 cent. de $\mathrm{mm}$. de longueur. Elle habite les eaux douces.

Le Navicula (Pinnularia) Tabellaria, Ehb, ressemble beaucoup au $N$. stauroptera, sauf que les renflements du milieu et des extrémités sont un peu plus marqués et que les eôtes au niveau du nodule médian sont réduites à des points. Cependant, il y a des variétés (N. Tabellaria stauroneiformis, V. H.) où elles manquent com- 
plètement et oủ les renflements sont très peu sensibles (1). Mais le frustule est relativement plus large que dans l'espèce précédente, les côtes sont plus serrées ( 12 a 10 dans 1 c. de mill.) et la taille du frustule est plus grande, 14 cent. de $\mathrm{mm}$. Eau douce.

Le Navicula (Pinn.) gibba, Kz. appartient encore à ce groupe. Le renflement médian se prolonge jusqu'aux renflements terminaux. Les còtes; au nombre de 12 dans le centième de millim., très courtes autour du nodule médian, ne manquent ordinairement pas tout à fait.

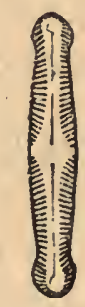

Fig. 174

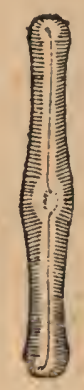

Fig. 175

Fig. 174. - Navicula (Pinn.) gibba, Kz (d’après Van Heurck).

Fig. 175. - Leméme d'après A. Truan). N. Tabellariu.

Plusieurs petites espèces d'eau douce se rangent encore dans ce groupe, caractérisé par la large bande hyaline le long du raphé et autour du nodule médian, telles que les :

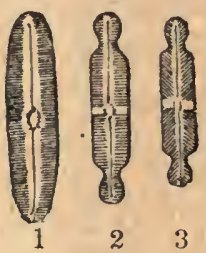

Fig. 176. - 1. Nav. (Pinn) viridis, var. commutet \& Gr.

2. Stauroneis anceps.

3. Nav. (Pinn.) bicapitata, Lag. (d'après A. Truan).

Navicula (Pinn.) bicapitata, Lagerst., jolie espèce aux còtes rayonnantes, aux extrémités capitées, arêc 10 à 12 còtes dans 1 cent. de mm. et une longueur de 6 centiemes de $\mathrm{mm}$.

(1) Cette forme parait plutôt un Navicula stauroptera qu'une variété du Na Tabellaria. 
Naricula (Pinn.) subcapitata, Greg., plus petit de moitié et dont les extrémités sont faiblement capitées.

Naricula (Pinn.) appendiculata, K\%, très petit (2 1/2 à 3 cent. de $\mathrm{mm}$.), aux còtes trés fines ( 16 à 17 dans 1 e. de $\mathrm{mm}$.), aux valves elliptiques longues aree des extrémités très peu ou pas eapitées ni rostrées. Le $N$. exilis, Kz. n'est qu'une variété à extrémités capitulées de cette espèce.

Navicula (Pin.) globiceps, Greg., et Nav. (Pinn.) Braunii Gr., petites espèces aux extrémités rostrées ou capitées, arec un renflement au milieu, globuleux chez le premier, fusiforme ehez le sccond. Cótes fines : 16 à 18 dans 1 cent. de mm. pour le premicr, 11 à 12 pour le second. Ete.

Pour compléter ce qui a rapport aux Pinnularićes mineures dont les valyes ne sont ni contractées au milieu, ni bi ou tri-ondulées sur les bords, ajoutons qu'il y a eneore un petit groupe d'espèces qui se distingue du préeédent par ec que les còtes attcignent presque le raphé, en laissant un faible espace lisse autour du nodule médian. Ce détail de structure change tout de suite l'aspect de ces Naviculées et les différencie immédiatement des précédentes.

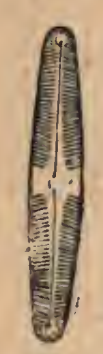

Fig. 17\%.

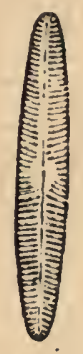

Fig. 178.

Fig. 177. - Nav. (Pinn.) appendiculatu, K\%, (gr. 900. diam. env.)

Fig. 178. - Nav. (Pinn.) retusa, Breb. (gr. 450 dian.)

C'est ainsi que le Navicula (Pinn.) sublinearis, Gr. ressemble au $N$. appendiculata, sauf l'absence de bande lisse le long du raphé, et les côtes qui sont beaucoup plus serrées; - ct que le Nav. (Pinn.) Itilseana, Jan, ressemble au Nav. bicapitata, sauf le mème détail relatif au raphé.

Le Naricula (Pinn.) relusa, Bréb. ressemble aussi beaucoup au Nav. appendiculata, mais sa taille est double, ses cótes sont un peu moins serrées et il est marin.

Toutes les espèces que nous venons de décrire appartiennent au groupe des Pinnulariées mineures à còtes serrées et à bords non 
eontractés au milieu ni bi ou tri-ondulés sur la face valvaire. Nous avons maintenant à signaler les cspèces dont les bords sont ondulés.

Le Navicula (Pinn.) mesolopta, Ehb., a les valves très ondulées avcc les extrémités rostrées-capitées. Les còtcs sont assez serrées (10 à 14 dans 1 cent. de mm.), laissant très pcu d'espace lisse le long du raphć et autour du nodulc médian. Elles sont un peu rayonnantes autour de ce nodulc. C'est unc asscz petite espèce d'cau douee, ayant dc 3 à 6 cent. de $\mathrm{mm}$.

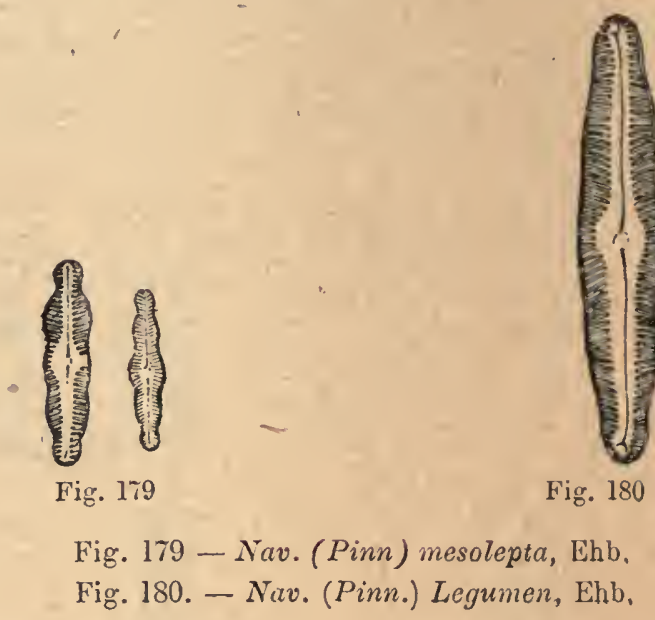

Le Navicula (Pinnularia) legumen, Ehb., cst plus grand, mesu. rant de 8 à 10 cent. de millim. Il a une forme analogue avec des ondulations moins accentuées et les cxtrémitès à peine capitées, comme unc gousse de légumineuse qui renfermerait trois graines. Il se distingue surtout par la large zone hyaline le long du raphé et autour du nodule. - Ses còtes sont nettement rąyonnantes. (Eau doucc).

Le Navicula (Pinn.) polyonca, Bréb., pourrait ètre considéré eomme un $N$. legumen très dilaté, globuleux au niveau du nodule médian; il est un peu plus petit.

Nous bornerons à ces détails ce que nous avons à dire des Naviculécs formant l'aneien genrc Pinnularia, d'Ehrenberg, caractérisé par la présenee de côtes plus ou moins robustes au lieu de stries perlées. Nous pouvons résumer de la manière suivante la division que nous avons opérće parmi les espèees :

1. Majeures : taille généralement considérable, de 10 à 50 eentièmes de millimètres. Còtes robustcs ct peu serrées : de $\breve{a} \dot{a} \mathbf{T}$, 
rarement 10, dans 1 cent de mill. (Nav. major, nobilis, viridis, cardinalis, etc).

2. Mineures : taille plus petite, de 2 à 10 ou 14 cent. de millim.

A. Còtes robustes et peu serrées ( 4 à 6 dans 1 c. de mm.) (Nar. lata, borealis, etc).

$B$. Còtes serrées (de 8 à 24 dans 1 c. de $\mathrm{mm}$ ).

* Bords sans contriction au milieu (sur la face valvaire) et non bi ou tri-ondulés.

a. Large zone hyalino le long du raphé et autour du nodule médian (N. Brebissonii, stauroptera, gibba, Tabellaira, bicapitata, appendiculata, globiceps, etc).

b. Pas de bande hyaline le long du raphé (N. sublinearis, Hilseana, retusa, etc).

* Bords bi ou tri-ondulés, ou resserrés au milieu (N. mesolapta, Legumen, etc.

Nous arrivons maintenant aux espèces dont les valves sont marquées de côtes ou de stries, plus ou moins fines, résolubles en perles ou grains. Ce sont les Navicula proprement dits, et nous allons retrouver parmi eux des formes nombreuses semblables à celles que nous avons rencontrées parmi les Navicules Pinnulariées, avec cette différence que les còtes ou les stries sont divisées en travers ou perlées, ou bien, dans quelques cas, que des cótes nọ résolubles sont alternées avec des stries perlées.

Les diatomistes ont établi des divisions assez nombreuses pour mettre de l'ordre parmi toutes ces espèces en les répartissant en plusieurs groupes; nous suivrons ce schéma d'une manière générale, en le simplifiant autant que possible. Néanmoins, nous n'hésitons pas à déclarer que nous ne trouvons pas toutes ces divisions suffisamment fondées. Établies souvent sur des caractères de détail, elles ont le tort de séparer, dans des groupes différents, des espèces que l'ensemble de leurs caractères, leur aspect général tendent au contraire à rapprocher. Il y a, à notre avis, parmi toutes ces Navicules, quelques formes types autour desquelles les autres se groupent naturellement, et ces formes typiques ne sont pas aussi nombreuses qu'on pourrait le croire.

Aussi n'avons-nous pas adopté généralement la méthode dichotomique qui, lorsqu'elle s'applique à un très grand nombre de sujets, est moins commode qu'on le dit et arrive souvent à créer des groupements artificiels tout à fait contraires à ceux que la nature a établis. 
Nous nous sommes borné à décrire les quelques types que nous considérons comme primordiaux, en les accompagnant des principales formes qui s'y rapportent et dont nous indifuons seulement alors les coractères différentiels. Nous trouvons ce systène, que nous considérons comme rationnel, plus commode que tout autre pour arriver à ranger une espèce donnée à la place qu'elle doit occuper dans une classification naturelle.

Les espèces rangées par M. II. Van Heurck dans le groupe des Radiosées ont encore l'aspect de Pinnulariées, parce que leurs valves portent de réritables còtes qui, au premier abord, semblent lisses comme celles des Pinnularia, mais examinées de plus près paraissent plus ou moins finement divisées en travers. Ce ne sont pas à proprement parler des stries perlées, formées de séries do perles ou de grains isolés et alignés dans certains sens : l'aspect est différent et, bien que difficile à définir, se reconnait très bien, ear on distingue facilement, sur les Naviculées qui montrent à la fois des côtes divisées et des stries perlées, ce qui est côtes et ce qui est stries.

M. H. Van Heurck répartit les Naviculées Radiosées en trois groupes, suivant la disposition de leurs côtes :

$1^{\circ}$ Côtes médianes rayonnantes, còtes terminales convergentes;

$2^{\circ}$ Còtes médianes rayonnantes, còtes terminales perpendiculaires au raphé.

$3^{\circ}$ Cótes rayonnantes partout, jusqu'aux extrèmités de la valve.

Dans le premier groupe, nous trouvons d'abord deux toutes petites espèces d'eau douce, les Navicula costulata, Gr. et $N$. humilis, Donk. qui ont été jusqu'ici décrites parmi les Pinnulariées, parce que leurs côtés n'avaient pas encore été résolues. C'est le $\mathbf{I}^{\mathbf{r}} \mathrm{H}$. Van Heurck qui a réussi à les résoudre dans le milicu à haut indice de M. H. I. Smith.

Elles ont toutes deux les côtes robustes et écartées (8 dans 1 cent. de $\mathrm{mm}$ ). Mais la première, $N$. costulata, a une forme lancéolée rhomboïdale, et la seconde, $N$. humilis, est renflée au milieu avec des extrèmités capitées. La première n'a pas de bandes hyalines le long du raphé, mais un large espace autour de nodule médian; la seconde a une bande le long du raphé un peu dilatée au nodule. La taille de ces deux espèces ne dépasse pas 2 c. de $\mathrm{mm}$. (Fig. 183, 1).

Toutes les autres espèces de ce groupe ont les côtes beaucoup plus facilement résolubles, bien que les petites lignes qui divisent 
les eòtes en travers soient généralement très délieates. Nous eilerons les suivantes.

Le Navicula oblonga, Kz.; belle espèee d'eau douee à valve longue et minec, très peu gonflée au nilieu et extrêmement peu aux extrêmités. Les còtes sont robustes : il y en a $\because$ au milieu, plus haut 7 , et aux extrèmités de la valve 8 , dans 1 eent. de millim. Ces eòtes qui, au niveau du nodule médian, sont divergentes du eòté des bords, deviennent convergentes à l'extrêmité de la valve, et, à une certaine distanee des nodules terminaux, se brisent dans leur direetion et, de droites qu'elles étaient, deviennent angulaires. Cette espèee, assez eommune dans les eaux douees, montre une zone hyaline le long du raphé avee élargissement au eentre, et des eòtes finement divisécs. Elle mesure de 15 à 18 eent de $\mathrm{mm}$.

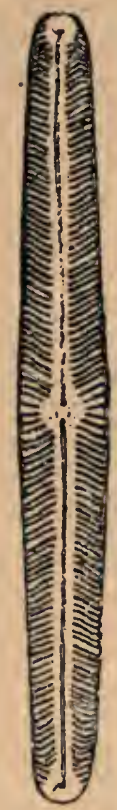

.Fig. 181.

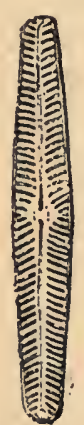

Fig. 182.

Fig. 181, - Navicula oblonga, Kz, d'après H. V. Heurck.

Fig. 182. - Le même, d'après A. Truan.

Le Navicula peregrina, $\mathrm{Kz}$, est relativement beaueoup plus large, avec des extrèmités plus atténuées. Il y a une étroite zone lisse le long du raphé et un espaee dilaté transversalemont autour du nodule médian. Les còtes sont espacées eomme dans l'espèee préeédente et se rapprochent de mème à mesure qu'elles sont plus près des extrèmités; mais elles ne forment à aucun niveau de ligne brisée. 
Cette espèce, qui vit dans les eaux saumàtres et qui a de 8 à 11 cent. de millim., fournit quelques variétés dont certaines vivent dans les eaux douces (N. peregrina minusculus, Schum).

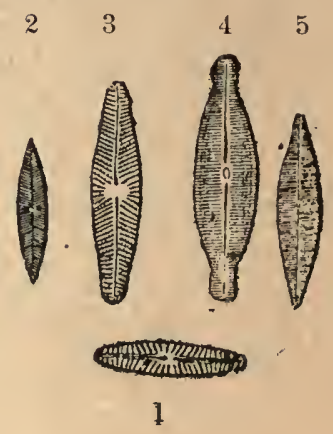

Fig, 183, - 1. Ncuviculà costulata, Gr.

2. Navicula gracilis, Ehb.

3. Navicula peregrina, Ehb.

4. Nuvioula producta, W. Sm.

5. Schironema amplius, Gr. (d'après M. Truan).

Les autres espcèes de ces groupes ont les côtes plus serrées : 10 en moyenne par centième de millim. Ce sont, par exemple :

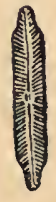

Fig. 184

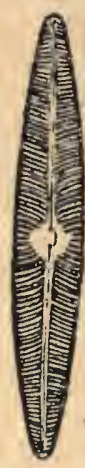

Fig. 185

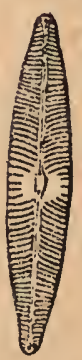

Fig. 186

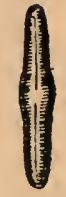

Fig. 187

Fig. 184. - 1. Navicula angusta, Gr.

Fig. 185. - Navicula radiosa, Kz.

Fig. 186. - 4. Nav. viridula, Kz.

Fig. 187. - Nav. viridula avenacea, Bréb.

Le Navicula gracilis, Kz., a les extrêmités en fer de lance. Toutes les côtes atteignent le raphé, les 2 ou 3 côtes médianes seulement 
sont un peu éeourtées et ne vont pas tout à fait jusqu'au nodule eentral. Il habite les eaux douees et sa taille varie de 4 à 8 cent. de mm. (2, Fig. 183).

Le Navicula radiosa, Kz., est une espèee d'eau douee assez eommune et d'une physionomie très earaetérisée. Les valves sont longues, laneéolées, pointues mème dans une variété (Pinnularia acreta, de W. Smith). Les còtes sont fortes, très nettement rayonnantes sur une grande étendue. de la valve, les terminales seulement eonvergentes. Toutes atteignent le raphé et laissent un petit espace lisse autour du nodule central. On en eompte 10 à 12 dans 1 eent, de millim. et le frustule mesure de 4 à 6 eent, de $\mathrm{mm}$. (Fig. 18.ั.) La variété Nav. radiosa acuta, W. Sm. est représentée (Fig. 208, 5, p. 272),

* Le Navioula viridula, Kz, a les extrémités plus obtuses, un peu rostrées; les eôtes sont eomme dans l'espèee préeédente, mais moins nettement rayonnantes, sauf à la partie médiane, atteignant le raphé mais laissant un large espace lisse autour du nodulo médian, C'est une espèce d'eau douee, longue environ de 7 cent, de mill, Elle fournit quelques variétés, dont une, le $N$. vir. avenacea, Bréb. a la forme d'un grain d'avoine. (Fig, 186 et 187.)

Les Navicula rhyncocephala, Kz., et $N$. cryptocephala, $\mathrm{Kz}$., sont plus petits, un peu plus larges, ont les extrémités nettement rostrées, les eòtes robustes atteignant le raphé et laissant une aire lisse autour du nodule médian; mais dans le premier, elles sont plus espaeées (9 à 12 dans 1 eent. de mm.) et plus fortement divisées en travers que dans le seeond, où elles sont serrées (16 env. dans 1 eent. de mm.), et très faiblement divisées.
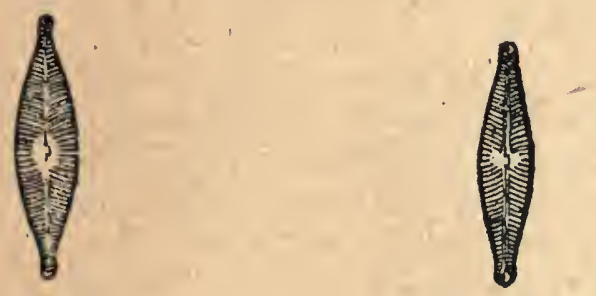

Fig. 188. - Nav. rhyncocephala, Kz. Fig. 189. - N. cryptocephala.

Le $N$. rhyncocephala vit dans l'eau saumàtre et mesure de כั à 6 cent de mm.; le $N$. cryptocephala habite les eaux douces et ne dépasse guère 3 eent. de $\mathrm{mm}$.

Le groupe des Radiosées à côtes- médianes rayonnantes et à eôtes terminales perpendieulaires au raphé, est peu nombreux. 
Nous citerons le Navicula cancellata, Donk, qui vit dans i'eau de mer ou l'eau saumàtre, et le $N$. Reinhardtii Grun, qui habite les eaux douces. Le premier a 6 ou 7 còtes; le second 9 à 10 dans 1 cent. de $\mathrm{mm}$. Ce sont des espèces à forme ellịptique ou lancéolée; mais le $N$. cancellata est nettement déprimé au niveau du nodule médian, de sorte que le frustule, vu par la face connective, est contracté au milieu. Leur taille varie de $31 / 2$ à 7 cent. de $\mathrm{mm}$.

Enfin, dans le groupe des Navicules Radiosées à còtes rayonnantes sur toute la lòngueur de la valve, nous signalerons les deux espèces suivantes :

Navicula dicephala, W. Sm, petite espèce d'eau douce, clliptique en nacelle, mais avec les deux extrémités rostrées-capitées. Les côtes atteignent le raphé mais s'écourtent au niveau du nodule médian : 9 à 11 dans 1 cent. de mm. - Longueur : de 2 à 4 cent. de $\mathrm{mm}$.
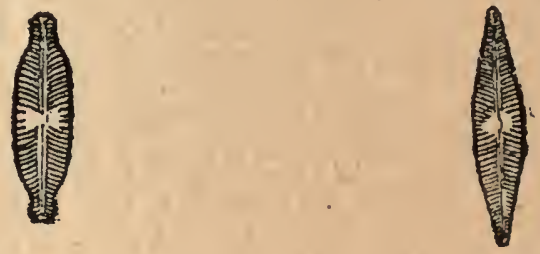

Fig. 190. -- Nav. dicephala W. Sm. Fig. 191. -- N. lanceolata, Kz.

N. lanceolata, $\mathrm{Kz}$; autre petite espèce d'cau douce, elliptique lancéolée, à extrémités atténuées, un peu rostrées, avec des còtes écourtées au niveau du nodule médian, atteignant le raphé partout ailleurs : 12 au milieu, 15 à 16 aux extrémités, dans 1 cent. de mm. — Longueur de 3 à $\check{~ c e n t . ~ d e ~} \mathrm{~mm}$.

On a réuni dans une sous-tribu dite des Didlymées (nous dirons plus loin ce que nous pensons de cette division), quelques fort belles espèces, la plupart marines, et qui se distinguent facilement à leur forme en guitare quand on les regarde par la face valvairc. Quelques-unes sont rayées de côtes à direction transversale, entre lesquelles sont des rangées de perles; d'autres ne présentent que les rangées de perles.

Parmi les premières, la plus remarquable est le Navicula crabro, Ehb., dont la valve a en longueur plus de quatre fois sa largeur médiane. Elle est étranglée en violon et marquée de fortes còtes perpendiculaires sur le raphé à la partie médiane, mais arquées sur les deux moitiés supéricure et inférieure de la valve, de manière à imiter les anneaux de l'abdomen de la guèpe : $31 / 2$ à 4 dans 1 cent. 
de $\mathrm{mm}$. Le raphé est droit, bordé d'une faible zone hyaline de eliaque eòté et au delà d'un sillon étroit, longitudinal. Le nodule médian est earré. Entre deux eòtes conséeutives, on voit deux rangées do petites perles. La longucur du frustule est de 8 à 12 cent. de mm.

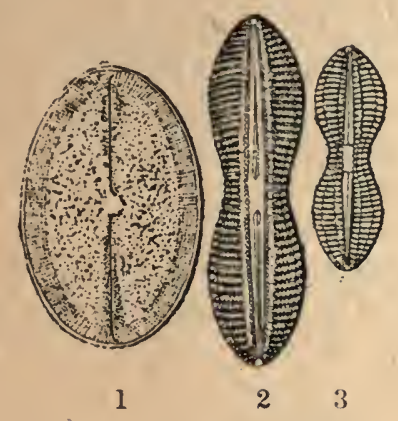

Fig. 192. - 1. Navicula protexta, Ehb.

2. Nav. Crabro, Ehb., Var. pandura, Bréb.

3. Nav. Crabro, Ehb., var. 'multicostata, Grun.

Cette espèce, marine, fournit différentes variétés, comme les $N$. Crabro pandura, Breb., et N. Crabro multicostata.

Le Navicula interrupta, Kz, marin aussi, ressemble au précédent, mais les demi-valves sont plus arrondies; les eòtes sont.un peu plus serrées et né eomprennent qu'une seule rangée de perles, difficiles à voir. Les sillons longitudinaux sont plus éloignés du raphé et interrompent les còtes, qui dans la partic médiane s'interrompent encore avant d'avoir atteint les bords latéraux de la valve. La longueur est de 7 à 8 cent. de mm.

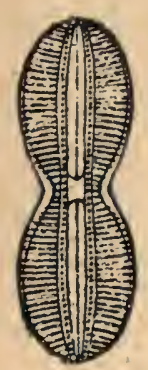

Fig. 193. - Navicu'a interrupta, K\%.

Parmi les Navieulées Didymées qui ne présentent pas de còtes, mais seulement des rangées de perles:, la plus commune est le $\mathrm{Na}$ vicula didyma, Ehb, espèce marine assez petite; peu contractíe au milieu, présentant de fines rangées transversales de grains, au. 
nombre de 8 environ, dans 1 cent. de mm. et deux protonds sillons droits de chaque còté et asscz éloignés du raphé. Sa longueur est de $\breve{3} 1 / 2$ cent. de $\mathrm{mm}$.

Le Navicula splendida, Greg., est plus grand, arec de forts sillons arqués, des rangées de points beaucoup moins serrées et qui, à la partie médiane, n’atteignent pas le bord externe de la valve.

Le Navicula bomboïdes, A. Schm., ressemble au précédent, mais les sillons sont brusquement dilatés à la partie moyenne et les stries, plus arquées, sont composées de groś grains quadrangulaires, qui se touchent presque comme des rangées de pavés. Il y a 4 à $\breve{b}$ stries dans 1 cent. de $\mathrm{mm}$., et le frustule mesure jusqu'à 11 cent de mm. - Marin.

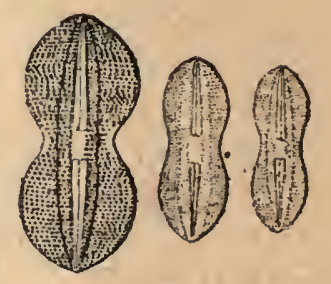

Fig. $191 \quad$ Fig. 195

Fig 194. - Navicula splendida, Greg.

Fig. 195. - Navicula didyma, Ehb.

La sous-tribu des Ellipticées rcnferme des espèces dont les valves sont elliptiques, et qui présentent des détails analogues à ceux qu'on observe chez les Didymées. Ce sont, pour ainsi dire, des Didymées qui ne sont pas contractées au milieu, sur la face valvaire. Elles ont les sillons longitudinaux le long du raphé, et les unes ont à la fois des côtes et des rangées dẹ perles, tandis que les autres n'ont que des rangées de perles.

Le Naricula Smithii, Bréb., est la plus belle espèce de ce groupe. Elle est elliptique dans sa forme générale, mais de contour variable, avec son raphé droit bordé d'une zonle hyaline, de larges sillons arqués, partant des nodules terminaux et plus ou moins éloignés du raphé.

Le nodule médian est grand et quadrangulaire; les nodules terminaux sont gros. Les còtes, au nombre de $\breve{b}$ dans 1 cent de mm., ne sont pas très fortes, mais soulignées par une double rangée de pétites perles. Cette espèce, marine, a de 9 à 10 cent. de $\mathrm{mm}$. de longuèur. 
Le Navicula fusca, Greg., ressemble au précédent, mais il est un peu plus grand, et n'a qu'un rang de perles, plus grosses, entre les còtes. Marin. - C'est aussi à ce groupe qu'il faut rapporter le Navicula Debyi, trouvé par le Dr J. Pantocsek, dans les dépòts fossiles de Hongrie.

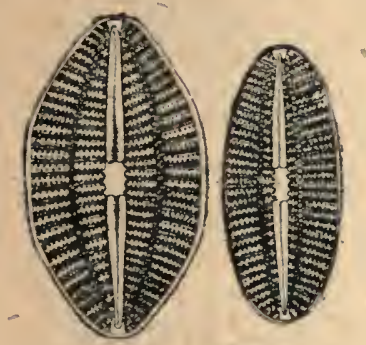

Fig. 196. - Navicula Smithii, Bréb. (2 exemplaires).

Parmi les Ellipticées sans còtes, nous citerons le Navicula elliptica, petite espèce en ellipse large, assez commune dans les eaux saumàtres et les eaux douces. Les sillons sont très rapprochés du

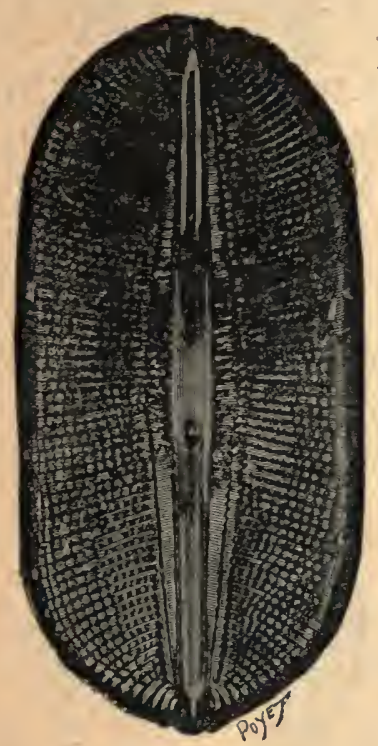

Fig. 197. - Navicula fusca, Greg., résolu à la lumière électrique par le $D^{r} H$. Van Heurck.

raphé, qui est bordé d'une zone hyaline élargie au nodule médian. Les stries, serrées (11 environ dans 1 cent. de $\mathrm{mm}$.), sont formées de grosses perles. Longueur de 2 à 3 cent. de $\mathrm{mm}$. 
La Navicula oculata, Brél)., se rapproche du précédent, mais il est plus petit encore et ses stries, à fines perles, sont beaucoup plus serrées : 17 dans 1 cent. de $\mathrm{mm}$. - Eau douce.

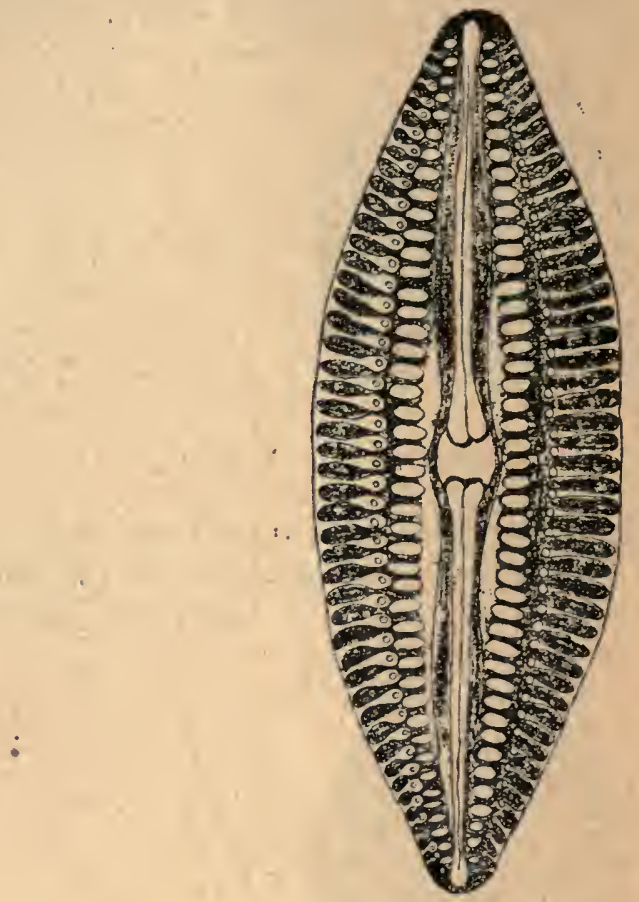

Fig. 198. - Navicula Debyi, Pantoc.

Nous pouvons placer à la suite des Naviculées Ellipticées les $\mathrm{Na}_{7}$ vicula protexta, Ehb. (Fig. 192, I) et $N$. Hennedyi,W. Sm., espèces marines, très jolies, mais rares. Dans la premiè re, les stries ne for-
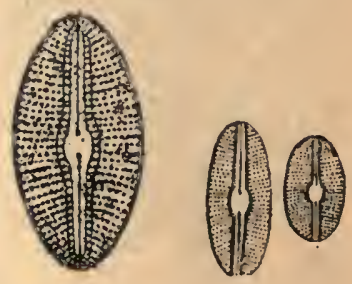

Fig. 199. - Ncviculn elliptica, Kz.

ment qu'une bande ou un cadre autour de la valve; elles sont à direction rayonnante, au nombre de 6 à 7 dans 1 cent. de mm., et formées de gros granules. Le long du raphé, les stries reparaissent compo- 
sécs de quelques gros points. Les sillons sont transformés en deux larges dépressions, demi-clliptiques, dont le fond est parsemé de points épar's, et qui coupent ainsi la striation en deux parties, l'une marginale, l'autre axiale. Le nodule médian est entouré d'une zone lıyaline. Vu par la face eonnective, le frustule est étranglé au milieu et la zone connective est striéc de 6 lignes longitudinales, formées de fins granules et disposées par groupes: une, deux, deux, une. Le frustule mesure de 7 à 9 eent. de mm. (1, Fig. 192.)

Le $N$. Hennedyi, W. Sm. est un peu plus petit, avee les stries plus serrẻes, formées de granules fins, et le fond des dépressions qui représentent les sillons lisses est trẹ̀s finement granulé.

Dans les espèces suirantes, les sillons longitudinaux prennent une autre disposition : ils forment une double eourbe, comme deux aceolades qui se regardent, et dont les milieux se rejoignent par un espace lisse, un stauros, autour du nodule médian; de sorte que leur cnsemble forme comme deux lyres, plus ou moins larges, opposées par leur base et dont le raphé représcinte les cordes. C'est ce qui a fait douner par Ehrenberg, le nom de Navicula lyra, à l'espèce type de ce groupe qu'on désigne sous le nom de Naviculées Lyrées.

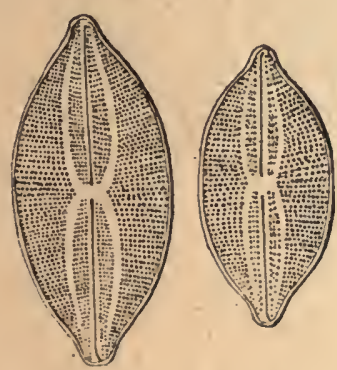

Fig. $200 \quad$ Fig. 201

Fig. 200. - Navicula lyra, Ehb.

Fig. 201. - Nıv. lyra, var. elliptica (d'après A. Truan).

Le Navicula lyra, Ehb. est une belle espèce marine et rare qui, outre la disposition des sillons que nous venons d'indiquer, présente une forme elliptique large, à extrémités un peu rostrées. Les stries arquices, eomme dans les précédentes espèces, autour du nodule médian comme centre, sont au nombre de 7 à 9 dans 1 cent. de $\mathrm{mm}$. et composées de granules; quelquefois, clles secontinuent, affaiblies, au fond des sillons qui, souvent aussi, sont lisses. Le frustule peut atteindre 12 cent. de mm.

Le Navicula forcipata, Grev., espèce marine moins rare que la 
précédentc, est plus petitc et a les branches des lyres plus droites, moins fermiées, un peu en forme de pince à sucre par suite de l'élargisscment en travers de leur base, c'est-ì-dire du stauros autour du nodule médian. Les stries sont beaucoup plus fines: 14 à $18 ̈$ dans 1 cent. de min. - La valve sèche est brune.

- Le $N$. abrupta, Greg., a les brançies des lyres ou des pinces brusquement intcrompucs avant d'atteindre les extrémités; il est elliptique sans rostration, et sa value sèche est hỵaline. Il y a 9 à 10 stries par cent. de mm. Espècc marine et rarc.

Le N. Truanii, Pantoc., trouvé par M. A. Truan dans le dépòt de Moron, en Espagne, et par M. J. Pantocsek (1), dans ceux de Szent-Peter, en Hongrie, appartient à ce groupe.

Nous ferons remarquer, avant d'aller plus loin, que toutes ces espèces distribuées dans les groupes des Didymées, Ellipticées, Prætextées (ou Hennedyées) et Lyrées peuvent se rattacher à un type commun qui, pour nous, serait le type Ellipticé. La forme généralc de la valve est une ellipse qui, dans les Didymées, subit unc dóformation par étranglement au petit axe, et une autre, dans les Lyrées, par un allongement rostré aux bouts du grand axe. Toutes présentent deux sillons longitudinaux sur leur valve, sillons qui prennent différents aspects et des formes diverses. Toutes ont un largé nodule médian qui sert de centre aux còtes ou aux stries des deux demi-valves supérieure et inférieure, strics qui figurent comme des ondes quasi-concentriques, et plus ou moins courbes, autour du nodule médian.

Il est évident que cet ensemble de caractères, très topiques malgré les modifications qu'ils éprouvent, constituc à toutęs ces espèces un certain " air de famille », qui permet de les rapprocher on un seul et véritable groupe naturel.

Nous placerons à la suitc des espèces que nous venons de décrire, un groupe composé de Navicules qui ont aussi un air de famille très évident. Comme les précédentes, elles ont une forme généralement elliptique, mais avec les extrémités pointues, en nacelle, ou bien terminées par une capitation ou une rostration obtuse qui en émousse la pointc. Elles ont toutes des stries fines, perlées, rayonnant plus ou moins autour du nodule central comme centre. Elles présentent toutes autour de ce nodule un large espace lisse, elliptique, naviculaire ou losangique, qui occupe quelquefois près de la moitié de la surface de la valve, réduisant les stries à une zone marginale. Nous pensons qu'on peut prendre le Navicula palpebralis,

(1) Pantocsek, Fossilen Bacillarien Ungarns, 1887. 
de Brébisson, pour type. Nous proposons donc de désigner sous le nom de Palpébiées les espèces qui composent cette sous-tribu (1).

Dans ce groupe, nous citerous plusieurs belles espèces, dont certaines présentent encore des sillons longitudinaux et font ainsi le passage de ces Navicules à celles du groupe précédent. Mais ces sillons, qui dans ces dernières avoisinaient plus ou moins le raphé, ont, dans le groupe qui nous occupe, émigré vers le bord des valves et sont presque marginaux. De plus, ils deviennent très étroits. Chez les autres, les sillons ont tout à fait disparu.

Parmi les éspèces qui présentent encore des sillons, nous signalerons les suivantes :

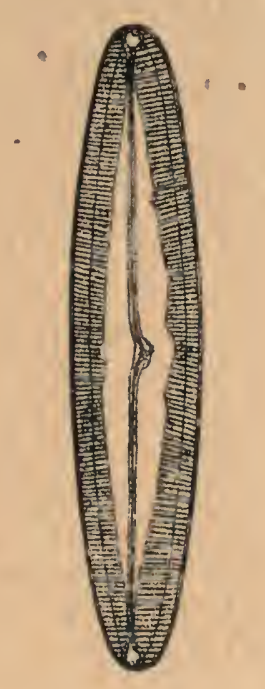

Fig. 202. - Nivicula formosa, Greg.

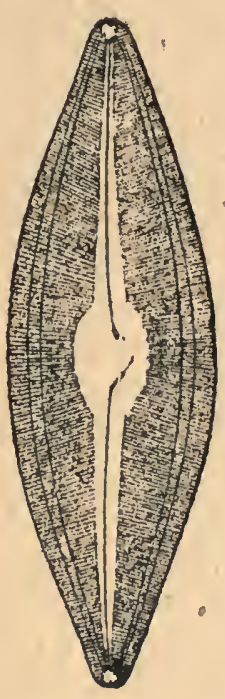

Fig. 203. - N. permagna, Bail.

Le Naricula formosa, Greg., belle espèce marine et d'eau saumàtre, dont la valve est elliptique, avec des extrémités atténuées mais obtuses. Le raphé est entouré d'une large bande hyaline, ${ }^{\circ}$ elliptique, avec un nodule médian latéral. Un sillon étroit, parallèle au bord latéral et encore éloigné de ce bord, coupe les stries vers leur moitié. Ces stries sont faiblement rayonnantes, et au nombre de 10 environ dans 1 cent. de $\mathrm{mm}$. Le frustule peut mesurer $14 \mathrm{c}$. de $\mathrm{mm}$.

(1) Nous y faisons rentrer les sous-tribus des Formosées, des Palpébraless, des Abréviées, des Perstriées, des Sculptées, des Affinées et des Limo. sées établies par M. H. Var' Heurck dans sa Synopsis. 
Le Navicula permagna, Bailey, a la même taille à peu près, mais il est un peu plus large, avec les extrémités plus aiguës. La bande hyaline s'étale en s'arrondissant autour du nodule médian. Les stries sont un peu plus serrées, et le sillon, plus près de la marge de la valve, parait double parce qu'il est eonstitué en réalité par une dépression dont on voit les deux lords. Cette espèce vit dans les eaux saumàtres.

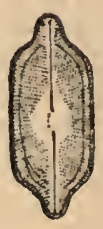

1

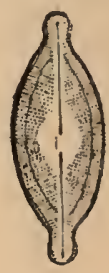

2

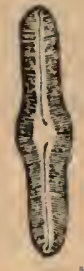

3

Fig. 204. - 1. Navicula umphisbana, Bory.

2. Naviculu subsulina, Donkiา.

3. N. limosa, Kz.

Le Naricula ampliisbœena, Bory, est une jolie espèee, très anciennement eonnue. Elle a la forme d'une ellipse quadrangulaire dont le grand diamètre est environ double du petit, mais aux deux extrémités de laquelle s'ajoute une rostration arrondie qui eoiffe le grand axe (raphé) eomme le bouton d'un fleuret. Le raphé est droit, avee un espace hyalin losangique, très large, autour du nodule médian qui est latéral. Un sillon parallèle au bord des valves, sub-marginal, eoupe les stries, qui sont nettement rayonnantes et au nombre de 14 dans 1 cent. de mm. Cette jolie espèee, très mobile, qui a 6 ou 7 cent. de mm. de long, vit dans les eaux douees ou légèrement salées.

Le Naricula subsatina, Donkin, réssemble eomplètement au précédent, sauf que les extrémités sont eapitulées, mais atténuées, obtuses, imitant l'angle interne d'un œil, et la Diatomée, regardée transver'salement, rappelle beaueoup la forme d'un œil vu de face. C'est de cette Navieule que nous voudrions faire le type de ce groupe. Mais elle est eonsidérée eomme une variété du $N$. amphisbcena, et elle fournit elle-même d'autres variétés. (Voir Pl. V.)

Le Navicula Bäimlerı, Pant., du dépòt fossile de Szent-Peter, est extrèmement voisin de $N$. subsalina et se présente tantôt avec un sillon sub-marginal, tantôt sans sillon.

Au nombre des espèees qui se rattaehent aux précédentes, mais qui n'ont plus de sillon sub-marginal, tout en conservant le large espace hyalin central, nous compterons : 
Le Navicula palpebialis, Breb., qui resscmble au $N$. subsalina, mais a les extrèmités tout à fait pointues, bien qu'il fourıisse une variété à extrémités obtuses légêrement capitées, avec nodule médian latéral. (N.palpebralis obtusa); il imite aussi la forme d'un xil. Mais outre qu'il n'y a pas de sillon, l'espace hyalin central est elliptique très long, ayant son bord parallèle aux bords mêmes de la valve. Les stries sont au nombre de 10 dans 1 cent. de mm. et le frustule ne mesure que $\check{3}$ cent. de mill.

Cette espèce fournit aussi des variétés, peu différentes 'du type. Une de ces variétés, cependant, a une tout autre forme; elle s'élargit carrément tout en conservant des extrèmités atténuées, obtuses, et établit un passage aux Navicula humerosa, $N$. gramulata, $N$. marina, N. breris, etc.

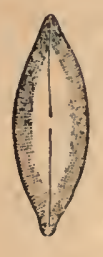

1

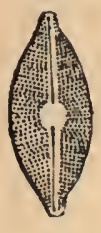

2

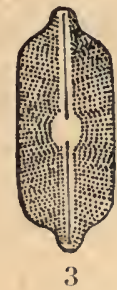

Fig. 205. - 1. Navicula xalpebralis, Bréb.

2. Nav. marina, Bréb.

3. Nav. granulata, Bréb.

Le Navicula humerosa, Bréb., a toujours le raphé droit des cspèces précédentes, mais sa forme est largement elliptique subquadrangulaire, arec extrémités brusquement atténuées obtuses, et l'espace hyalin central est considérablement diminué, dilaté non plus en long, mais en travers. Les strics, rayonnantes, composées de gros grains, sont aủ nombre de 9 environ dans 1 cent. de mill. La valve sèche est incolorc. Cette cspèce, marine, atteint 7 cent. de $\mathrm{mm}$. de long.

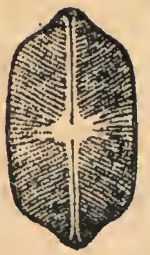

Fig. 206. - Navicula tumerosa, Bréb.

Le Navıcula granulata, Bréb., ressemble beaucoup. au précédent; l'espace hyalin central, assez restreint, est élargi transversa- 
lement, les stries sont un peu moins serrées et les grains plus gros. La valve sèclie est d'un bleu purpurin. La taille est un peu plus grande ( 7 à 9 cent de $\mathrm{mm}$ ). Espice marine.

Le Navicula marina est nettement elliptique, large, avec des extrêmités un peu atténuées obtuses. L'espace hyalin est plus petit encore, les stries plus serrées (10 à 11 dans 1 cent. de mm). Lo frustule a de 6 à 7 centièmes. Espèce marine.

Le Narzcula brevss, Greg., dérive comme forme du précédent. L'ellipse est plus allongée, l'espace hyalin plus grand, nettement arrondi; les stries beaucoup plus fines (14 dans 1 cent. de $\mathrm{mm}$ ). C'est une espèce marine de 6 à 7 cent: de $\mathrm{mm}$.

Cette espèce est encore une forme de passage et se rallie aux Naricula palpebralis et même au $N$. amphisbcena, car le $D^{r} H$. Van Heurck dit qu'elle présente sourent des sillons longitudinaux.

Du reste, toutes ces espèces donnent un grand nombre de variétés qui les rattachent manifestement les unes aux autres.

C'est ainsi qu'on peut encore rapprocher des espèces précédentes celles qui suivent; elles ont évidemment une affinité avee le tỹpo amphisbcena et leurs caractères se modifient graduellement pour passer à un autre type.

Le Naticula sculpta, Elbb, a la valve elliptique avec extrémités atténuées obtuses, en forme d'œil, semblable à cẹlle des $N$. palpebralis ou subsalina, un raphé droit bordé d'une ligne hyaline, et, de chaque côté, une large dépression régnant sur toute la longueur de la valve et qui interrompt les stries de maniêre à ne laisser qu'un seul rang de perles le long du raphé ; les stries reparaissent du còté du bord, n'ayant plus guère que la moitié de leur longueur normale. Elles sont très peu rayonnantes, formées de grosses perles et serrées (15̆ à 16 dans 1 cent. de mill). Sur l'un des cótés de la valve, il y a aussi une dépression transversale, perpendiculaire au raphé, au niveau du nodule médian, et allant jusqu'au bord de ce côté. Au fond de cette dépression les śtries marginales sont affaiblies. Cette espèces vit dans les eaux douces et saumàtres, et mesure de 7 à 8 cent. de millim.

Le Navicula sphœrophora, $\mathrm{Kz}$, a une forme analogue, mais les extrèmités sont ptus capitées, comme dans le $N$. amphisbcena: il est relativement un peu moins large que le précédent; il présente la même dépression transversale, unilatérale,-lisse; mais la dépression longitudinale, qui dans le $N$. sculpta règne de chaque côté du raphé, est remplacée par plusieurs dépressions étroites ou rides parallèles, dans le creux desquelles les stries manquent, tandis qu'elles persistent sur les crêtes, disposition qui aligne les perles 
sur le milieu de la valre en séries longitudinales. Ces stries, qui reparaissent entières du còté marginal, sont extrèmement peu rayonnantes, presque parallèles et serrées ( 16 dans un cent. de $\mathrm{mm}$ ). C'est une espèce d'eau douce, de 6 à 8 cent. de $\mathrm{mm}$. de long.

Cette espèce présente cette particularité, et nous penchons à croire que les espèces voisines la partagent plus ou moins, d'avoir l'endochròme disposé en une sẻule lame, comme chez les CyyBrLLÉEs (P. Petit); comme le $N$. sculpta, elle présente une certaine asymétrie en raison de l'absence de stries médianes sur un côté de la valve. On peut donc la considérer comme une forme de passage des Naviculées aux Crabellés. (Voir Pl. V.)

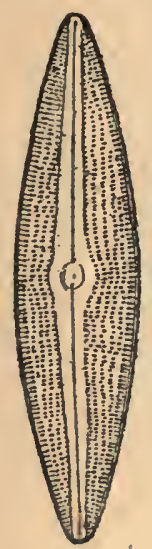

Fig. $20 \tau$ - Navicula serians, Bréb.

Dans le Navicula serians, Bréb., nous trouvons une valve elliptique longue, nettement naviculaire, dans laquelle le raphé est bordé d'une bande hyaline élargie autour du gros nodule médian. Il n'y a plus de dépression transversale, et les dépressions longitudinales sont devenues des sortes de rides très peu profondes, ou d'espaces qui segmentent les stries sur toute la valve et alignent les perles en quatre ou cinq séries longitudinales ondulées. Les stries sont très peu rayonnantes, composées de grains allongés transversalement, fines et serrées ( 24 dans 1 cent. de $\mathrm{mm}$ ). Vu par la face connective, le frustule est assez large et dilaté au milieu. Cette Navicule habite les eaux douces, les marais tourbeux, et atteint 8 cent. de $\mathrm{mm}$. de longueur.

A còtẻ de cette espèce s'en placent plusieurs,' comme le Navicula exilis, Grun., dans lesquelles les stries deviennent de plus en plu fines, les rides disparaissent; l'espace hyalin le long du raphé et autour du nodule devient de plus en plus petit, les extrémités pren- 
nent quelquefois une forme capitée ou sub-capiteee. Ces transformations successives nous amènent aux espèces suivantes.

Le Navicula cuspidata, Kz; la valve est lancéolée naviculaire, à peu près quatre fois aussi longue que large, avec les extrémités atténuées, presque pointues. Toute la valve est couverte de stries atteignant presque le raphé, elles sont à peu près perpendiculaires au raphé, par conséquent parallèles et non rayonnantes, au nombre de 14 dans 1 cent. de mm., coupées en travers par de fines stries longitudinales plus serrées. C'est une élégante espèce d'eau douce, assez communc, et qui peut atteindre jusqu'à 14 cent. de mm, (J. Brun) (Fig. 208. 1.)

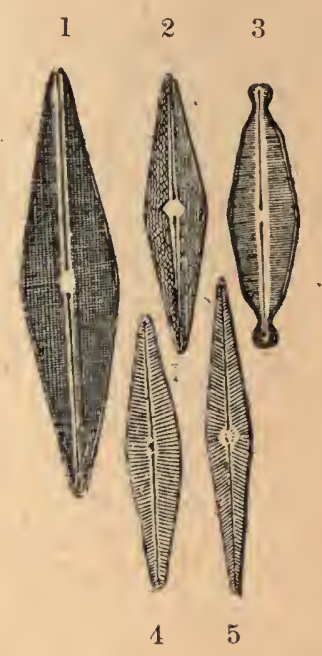

Fig. 208. - 1. Navicula cuspidata, Kz.

2. Navicula serians, var. minor, Gr.

3.'Navicula àmbigua, Ehb.

4. Schizonema floccosum, Kz.

5. Navicula rádiosa, var. acuta.

Le Navicula ambigua, Ehb. est considéré par le $\mathrm{D}^{\mathrm{r}} \mathrm{H}$. Van Heurck comme une variété plus petite, avec des extrémités prolongées et plus ou moins capitées. Les stries sont parallèles, perpendiculaires au raphé (16 à 19 dans 1 c. de mm. (Fig. 208, 3.)

Le Navicula iridis, Ehb., est une grande espèce d'eau douce, en forme de navette, "six fois aussi longue que large, dont le raphé, droit, a les bouts centraux tournés en crochet en sens opposés, avec une bande hyaline élargie autour du nodule médian un peu oblique. Les stries sont fines ( 16 env. dans 1 cent. de mm.), presque 
parallèles, formées de perles allongées et interrompues près des bords par un sillon submarginal profond. Sa longueur atteint 17 cent. de $\mathrm{mm}$.

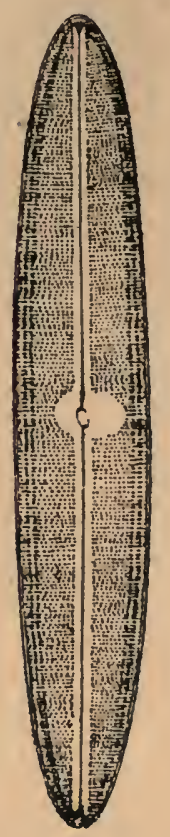

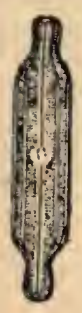

1

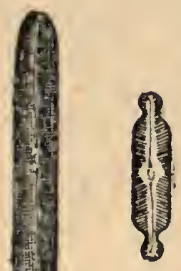

3

Fig. 209. - Nav. Iridis, Ehb. Fig. 210. - 1. Nav. amphirhynchus, Ehb. 2. Nav. firma, $\mathrm{Kz}$

3. Nav. bicapilata, Lag.

Le Navicula firma, $\mathrm{Kz}$, est une variété plus petite de cette espèce avec 23 à 28 stries dans 1 cent. de $\mathrm{mm}$.; ainsi que le $N$. amphigomphus, Ehb., belle forme d'eau douce aussi, qui a 18 stries dans 1 cent. de mill. et peut atteindre plus de 9 cent. de $\mathrm{mm}$. en longueur.

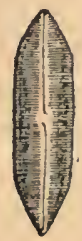

Fig. 211. - Nuv. Iridis amphigomphus, Ehb.

Le Dr H. Van Heurck donne aussi le Navicula producta, W. Sm. comme une varièté dụ Navicula Iridis. Cette variété 
revient à la forme d'ellipse avee les deux eapitations extrèmes et les deux sillons submarginaux, Les stries sont perpendieulaires au raphé et formées de grains allongẹ́s. (4, Fig. 183, p. 258.)

Le Navicula amphirhynchus, Ehb., est cneore, pour le Dr H. Van Heurck, une variété de N. Iridis, remarquable par ses extrémités largement rostrées, tandis que le Navicula affinis, Ehb. reproduit pour sa forme le Navicula producta et le type amphistöna.

Toutes ces espèces, d'eau douce, dérivent, quant à la forme, du type amphisbona. Les stries deviennent de plus en plus parallèles entre elles; perpendieulairement au raphé, et les sillons dégénèrent peu à peu en des lignes longitudinales qui eoupent les stries, deviennent à leur tour de plus en plus nombreuses et serrées, et forment des stries longitudinales qui chez le Navicula producta sont au nombre de 14 dans 1 cent. de millimètre.

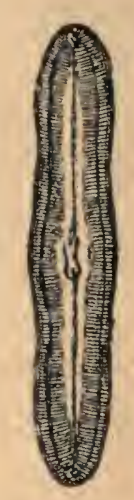

Fig. 2i2. - Nav. limosa, Kz.

C'est ainsi 'que, graduellement, nous arrivons d'une part au Navicula limosa et à ses congénéres, d'autre part au Navicula bacillum et à sẹ analogues.

Le Navicula lmosa, Kz. est une jolie espèce d'eau douce, de forme bacillaire allongée, renflée au milieu comme certains Pinnularia. Le raphé est toujours droit, entouré, sauf aux extrémités, d'un espace hyalin elliptique très allongé, avee un nodule médian un peu latéral et des stries presque perpendiculaires au raphé, sauf dans le milieu où elles sont un peu rayonnantes autour du nodule central. Il y en a 16 à 17 dans 1 cent. de millim; mais elles sont coupées non loin du bord par un sillon parallèle à ce bord. Le frustule a 7 à 8 cent. de mm. de larg. (Fig. 204, 3, p. 268 et Fig. 212.)

Le Navicula rentrıcosa, Elıb. ne nous parait ètre qu'une variété du précédent, ear tous les détails de strueture sont absolument iden- 
tiques, et la différence ne porte que sur la dilatation médiane des valves, dilatation qui est moins marquée et se fait d'une manière progressive, le frustule, vu par sa face valvaire, ayant une forme presque eomplètement bacillaire.

Le Navicula bacillais, Greg., a une forme tout à fait bacillaire, surtout dans la varićté Nav. bacillai is the'malis, Grun., et présente des stries partout perpendiculaires au raphé, parallèles par conséquent, fines et serrées, mais manquant souvent sur un des eòtés de la valve au niveau du nodule médian, disposition qui reproduit ee que nous avons déjà vu chez ies Navicula sculpta, sphcerophora, etc.

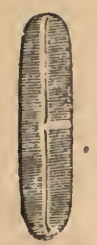

Fig. 213. - Nav, bacillaris, var, thermalis, Grun,

Avee le Navicula liber, W. Sm., nous arrivons à une forme tout à fait bacillaire, avec des stries exactement perpendiculaires au raphé, atteignant presque le raphé et laissant un espace hyalin étroit, elliptique, autour du nodule médian. Les nodules terminaux, très marqués, sont assez éloignés des extrémités, et les stries extrêmes rayonnent autour d'eux eomme dans beaueoup de Pinnulariées. Un sillon, partant de ees nodulss, suit les bords de la valve en s'arquant un peu au niveau du nodule médian. Les stries sont fines, serrées (18 dans 1 eent. de mm.). L'espèce est marine et longue d'environ 8 centièmes.

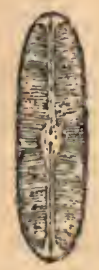

Fig. 214. -- Navicula bacillum, Ehb.

Enfin, le Navicula bacillum, Ehb., représente un bàtonnet court, 3 fois $1 / 2$ aussi long que large, avee des stries très faiblement rayonnantes, mais plus serrées aux extrémités qu'au eentre (17 aux extrémités, 14 au centre, dans 1 eent. de mm.) 
Pour terminer ee que nous avons à dire sur le genre Navicula nous n'ajouterons que quelques mots relatifs à une série de très petites espèces que le $\mathrm{D}^{\mathrm{r}} \mathrm{H}$. Van Heurek réunit sous le nom de Minutissimées. La plupart ne dépassent guère 2 eent. de millinı. en longueur et beaueoup n'atteignent pas eette dimension. Tels sont les Navicula atomus, Nægeli; N. atomoüdes, Grun.; N. minima, Grun.; $N$. binodis, W. Sm.; $N$. exilissima, Grun. Ces deux dernières espèces sont intéressantes en ce qu'elles eonstituent des « tests » assez diffieiles. Le $N$. binodis présente 30 stries, et le $N$. exilissima 40 stries environ dans 1 cent. de millim., très délieates et peu visibles, mème aree les objectifs homogènes. (H. V. Heurck.)

Nous n'avons pu, dans cet examen rapide de ce nombreux genre, que signaler les prineipales espèees; mais on roit qu'on 'peut les répartir en quelques groupes qui se distinguent aisément par des earactères dominants, lesquels, en se modifiant, donnent des espèees de passage. On ne peut, elı effet, s'empècher de reconnaitre eertains types importants, autour desquels se groupent, par des variations diverses portant tantôt sur un earactère, tantôt sur un autre, des séries plus ou moins nombreuses de formes pour ainsi dire dérivées, et quelques autres un peu aberrantes.

Nous avons indiqué plus haut les divisions qu'on peut établir parmi les espèees Pinnulariées et Radiosées; parmi les autres, on roit eombien celles qui appartiennent aux types crabro (ou didyma), elliptica, lyra, se rapproehent d'une manière étroite et passent aux types suivant : amphisbana, serians et bacillaris qui, eux aussi, sont alliès de très près. Beaucoup d'espèees exotiques ou très rares, que le eadre de eet ouvrage ne nous a pas permis de signaler, trouveraient toutes à se plaeer auprès de l'un ou de l'autre de ces types originaux.

Parmi les types que nous eonsidérons eomme aberrants, nous devons eiter le Navicula aspera, Ehb., pour lequel M. H. Van Heurek a eréẻ un groupe à part, eelui des Aspérées, à la suite des Naviculées lyrées, dont le type est le Navicula Lyra, Ehb. Nous pensons, comme nous l'expliquons plus loin, que eette espèce doit ètre distraite de ce genre, et nous l'arons jointe aux Stauroneis comme faisant le passage au genre Scoliopleura.

\section{§ 3. - Vankeurchia, Schizonema, Mastogloia, Diadesmis.}

A la suite du genre Navicula proprement dit se placent divers autres genres qui s'y rattachent de très près et offrent avec celui-ci, 
entre eux, et avec les genres voisins, de nombreuses formes de passage.

Vanheurckia. - Tel est, par exemple, le petit genre Vanheurckia, dédié par M. de Brébisson au savant botaniste d'Anvers, le $\mathrm{D}^{\mathrm{r}} \mathrm{H}$. Van Heurck. Ce genre est composé de quelques espèces d'eau douce, remarquables par la finesse de leurs stries, et qui constituent des tests intéressants. On trouve "quelquefois les frustules réunis dans des tubes, comme les Schizonema et les Collelonema; il faut done les considérer comme ayant un thalame tubulaire qu'ils abandonnent de bonne heure. En réalité, ce sont des Schizonema, plutòt que des Naricula.

Ces espèces ont les valves elliptiques lancéolées avec des stries parallèles, quelquefois seulement un peu rayonnantes autour du nodule médian, atteignant le raphé qui forme une double ligne droite. Le nodule médian est plus long que large et les nodules terminaux, compris dans la double ligne du raphé, sont assez éloignés des extrémités.

Tel est le Vanheurckia \%homboïdes, Bréb., le Naricula rhomboüdes d'Ehrenberg, jolie espèce qui vit dans les eaux douces, les tourbières, et atteint 6 à 7 cent: de millim. Ses stries sont très délicates, environ 24 à 28 dans un centième de millim.; clles sont formées de fines perles qui s’alignent en stries longitudinales. Les valves sont un peu resserrées au-dessous des extrémités.
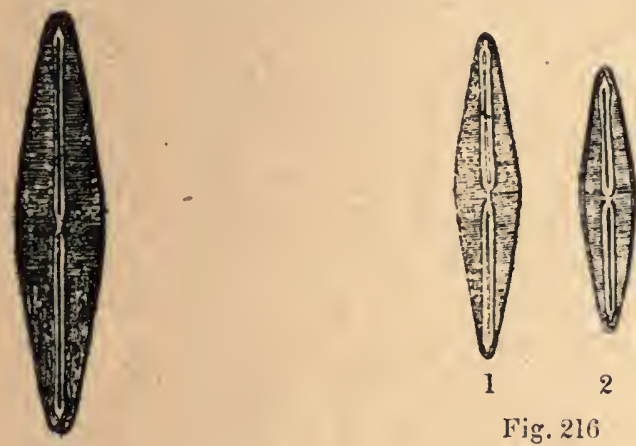

Fig. 216

Fig. 215. - Vanheurckia rhomboïdes, Breb. (1)

Fig. 216 - 1. Vanheurckia vhomboïdes, Bréb. 2. Vanh.crassinervis.

Lo Vanheurchia crassineris est le Naicula crassinerva de

(1) La partie supérieure est'résolue en stries transversales, et la partie inférieure en stries longitudinales formées par l'alignement des perles composant es stries. 
Brébisson, le Frustulua saxonica de Rabenhorst; e'est un test célébre, un peu plus petit que le préeédent (ふ eent. de millim.) dont il n'est qu'une variété. Ses stries sont très fines et très difficiles à résoudre: 34 à 33 dans 1 eent. de $\mathrm{mm}$.

Le Vanheurckia vuidula, Breb., est un Schizonema viridulum. Brébisson en avait d'abord fait un Colletonema rividulum. On trouve souvent les frustules réunis dans des tubes. Il atteint jusqu'à 11 eent. de millim. et présente 28 à 30 stries, finement perlées, dans 1 cent. de millim.

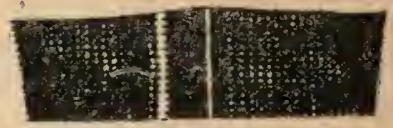

Fig. 217. - Vanh. rhombioides, fragment résolu en perles sous un gross. de 1800 diamètres, d'après une photog. du $\mathrm{D}^{\mathrm{r}}$ Woodward.

Enfin, le Vanheurckia vulgaris, V. Heurck, est le Colletonema vilgare de Thwaites. Il a les bords plus arrondis, les extrémités plus obtusés, les lignes du raphé éeartées, interrompues avant d'atteindre le nodule médian. Les stries médianes rayonnent un peu; elles sont plus fortes et plus écartées (24 dans 1 cent. de mm.) que les ștries terminales (34 dans 1 ecnt. de $\mathrm{mm}$.) Cette espèce, d'eau douce, ne dépasse guère 5 cent. de $\mathrm{mm}$.

Schizonema. - Le genre Schizonema est composé de Navieules qui, à l'état jeune au moins, ont leurs frustules renfermés dans des tubes mucilagineux, comme les Encyonema, qui appartiennent à la tribu des Cymbelidées. Nous y rattacherons l'ancien genre Colletonema, qui possède aussi un thalame tubulaire, et qui établit plus particulièrement eneore un passage aux Encyonema, parce que les valves ne sont pas tout à fait symétriques par rapport au grand axe. Les Schizonema sont ordinairement des espèces marines à valves peu silieifiées. Ils ont un raphé droit où, dans certaines espèces, on peut reconnaitre une double ligne. Les stries sont à direction généralement parallèle, quelquefois un peu rayonnantes autour du nodule médian; clles atteignent le plus souvent le raphé, mais non le nodule médian, autour duquel règne parfois une zone hyialine.

L'une de ces espèces, le Sch. Smithii, C. Ag., possède de véritables eôtes ou du moins des stries très fortes, relativement espacées (13 dans 1 cent, de mm.), finement divisées, rayonnant dans la partie médiane, laissant une étroite bande elaire le long du raphé et une 
zone élargie autour du nodule médian, et convergeant vers les bords de la valve aux cxtrémités. L'espèce mésure 6 cent. de mm. de long.

Le Schizonema crucigemum, W. Sm., est très allongé et très étroit avec les extrémités aiguës. Les stries sont serrées ( 24 dans 1 cent. de mm.), presque parallèles, divisées en perles qui s'alignent en stries longitudinales très fines. Pas de bande hyaline le long du raphé, mais un espace élargi autour du nodule médian, espace qui porte dcux fortes stries. La longueur du frustule varie de 7 à 8 eent. de $\mathrm{mm}$.

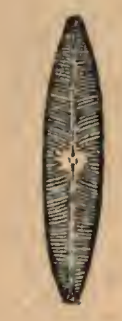

Fig. 218

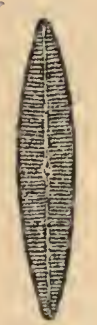

Fig. 219

Fig. 218. - Schironema Smithii, C. $\Lambda$ g.

Fig. 219. - Sch. ramosissimum, C. Ag.

Le Schizonema ramosıssmm les valves sont couvertes de stries perlées, sans espace lisse, dont les divisions s'alignent en stries longitudinales. Il y a environ 14 stries dans 1 cent. de $\mathrm{mm}$. et le frustule mesure : centièmes de long. - Le Sch.floccosum, Kz., est une forme voisine.(4, Fig. 208, p. 272$)$.

Le Schizonema amplius, Grun., est une espèce un peu plus forte, de même forme, mais à stries plus grosses, dont les divisions forment aussi des lignes longitudinales. ( $\%$, Fig. 183, p. 258.)

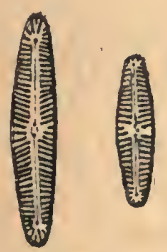

Fig. 220 - Schizonemu lacustre, C. Ag.

Le Schizonena lacustre, C. Ag., est le type de l'ancien genre Colletonema, dont les frustules se forment aussi dans des tubes mais ne sont pas toujours complètement symétriques par rapport à la ligne médiane. Dans l'espèce qui nous occupe, ainsi que dans 
celles qui s'y rattachent comme les Schizonema (Colletonema) neglectum, Thw., Schizonema (Collet.) Thwaitesii, Grun., les stries sont plus grosses, atteignant le raphé sauf au niveau du nodule médian où, plus courtes ou plus espaećes, elles laissent un espace lisse élargi transversalement. Ces stries sont très rayonnantes autour du nodule médian et autour des nodules terminaux qui sont plaeés loin des extrémités. Il n'y en a quc 9 dans 1 cent. de $\mathrm{mm}$. ; elles sunt fincment divisées en travers. C'est une espèce d'eau douce longue de 3 à 5 cent. de num.

Nous passerons rapidement sur plusieurs petits genres qui se placent à la suite de ceux que nous venons de décrirc et qui présentent moins d'intérêt, étant pcu riches en espèees, assez rares d'ailleurs ẹt souvent peu connues.

Mastogloia. - Le genre Mastogloia, Thwaites, sc distingue par un caractère très eurieux. Il se compose d'assez petites espèees, de forme navieulée elliptique, dont les vilves présentent à l'intérieur, le long de leurs bords, une rangée de grosses eellulcs formées par des lames silieeuses intcrnes adhérentes à la faee profonde des

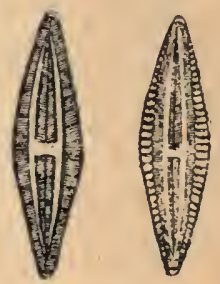

Fig. 221. - Mastogloia Braunii, Gr.

valves. La surface supérieure de celle-ci est marquée de stries ordinaircment assez fines.

Ces espèees vivent dans l'eau douce ou l'eau saumàtre ; les frustulcs se forment au sein d'une masse gélatineuse plus, ou moins volumineuse.

L'une des plus remarquables est le Mastogloia Braunii, Gr., dont les cellules ou logettes sont nombreuses; les stries, finement ponetuées, au nombre de 18 dans 1 eent. de $\mathrm{mm}$., un peu raxonnantes, sont interrompues par une ligne lisse se réunissant au nodule - médian dc manière à former un dessin en double lyre, comme dans lc Navicula lyra.

Les autres espèees ne présentent pas ee sillon lyré; tels sont les Mastoglora Smithii, Thw., M. lancealata, Thw, M. exigua Lew., dont les bords sont courbes, de sorte que la rangée des eel- 
Iıles qui les ourle est en ligne courbe aussi; les deux premières espèces ont des cellules nombreuses, la dernière n'en a souvent que trois (H. van Heurck). Les Mastogloia Dansei, Thw. et M. Grevillei, W. Sm., ont les còtés assez rectilignes, dans une partie de leur étendue, pour que la rangée de cellules, qui ne borde que cette partie, soit elle-mème rectiligne.

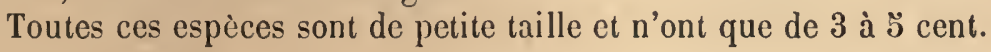
de mm. de long.

Diadesmis. - Les Diadesmis, Kz, sont des espèces encore plus petites, caractérisées surtout par ce que les frustules restent réunis les uns aux autres en longs rubans, sans être enfermés dans des tubes. Celles que nous connaissons habitent les eaux douces. Une de ces espèces présente une disposition qui rappelle les Mastogloia, c'est-ì-dire que le long des bords règne une rangée de grosses perles, mais ce ne sont pas des cellules placées sous la valve. Il s'agit du Diadesmis gallica, W. Sm., qui montre, en outre, des stries très fines, un peu rayonnantes, au nombre de 28 dans 1 centième de mill. Le frustule est elliptique allongé, à extrémités obtuses, et un peu élargi au milieu. Il y a un petit espace lisse le long du raphé et autour du nodule médian.

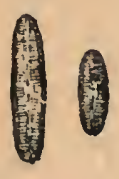

Fig. 222.

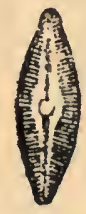

Fig. 223.

Fig. 222. - Diadesmis gallica. W. Sm. (1000 diam.)

Fig. 223. - Diad. confervacea, Gr. (1000 Diam).

Le Diadesmis confervacea, Gr., est une jolie petite espèce à strics rayonnantes, avec bande hyaline et aire lisse autour du raphẻ et du nodule médian.

Ces espèces ne dépassent guère 1 cent. de millimètre $1 / 2$. 


\title{
DESCRIPTION DES PRINCIPALES ESPECES (Suite)
}

\author{
NAVICULÉES (Suite) \\ $\S 1$. - Stauroneis
}

Le genre Stauroneis a été créé en 1843, par Ehrenberg, pour des Naviculées dans lesquelles le nodule médian s'est dilaté transversalement jusqu'aux deux bords des valves en un large espace

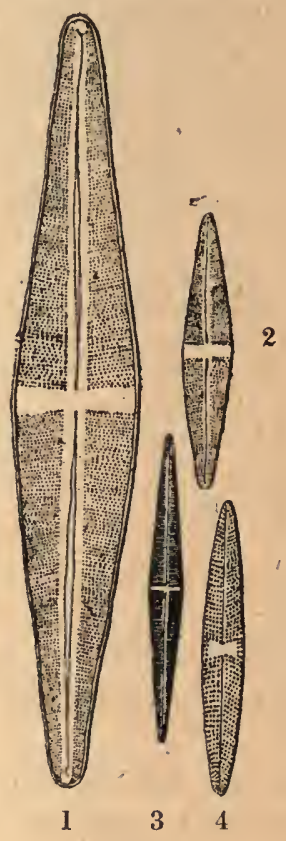

Fjg. 224. - 1. Stauroneis phonicenteron, Ehb.

2. Stauroneis gracilis, W. Sm.

3. Stauroneis spicula, Hickie.

4. Stauroneis aspera, Ehb.

lisse ou stauros. Comme le raphé est le plus souvent bordé d'une bande hyaline plus ou moins large, la valve parait marquée d'une grande croix suivant ses deux axes. Les valves sont couvertes de stries perlées.

Les deux lames d'endochròme qui forment les chromatophores 
des Stanioneis sont toujour's ondulées sur leurs bords, ce qui distingue ees espèees de celles du genre Navicula.

Le type de ee genre est le Stauroneis phonicenteron, Ehb., ainsi nommé paree que son eentre parait souvent rouge, par un jeu de lumière diffractée. Il a une forme laneéolée longue, progressivement atténuée vers les deux extrémités, qui sont obtuses arrondies. Le raphé est droit, formé de deux lignes qui se réunissent en atteignant les nodules terminaux et le nodule médian, bordé d'une bande hyaline assez large qui se croise avee le stauros résultant de la dilatation du nodule médian. Ce stauros est ordinairement plus large sur les bords de la valve qu'au eentre. Les stries sont délieates, finement perlées, légèrement rayonnantes, au nombre de 11 environ dans 1 eent. de mm. C'est une espèee d'eau douee, assez eommune, et longue de 10 à 17 eent. de mm.

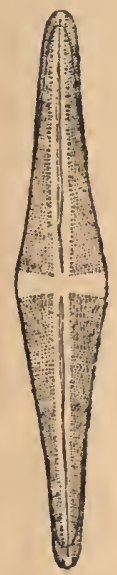

Fig. 225. - Stauroneis acuta, W. Sm.

Le Stauroners acuta, W. Sm., a les valves plus aiguës aux extrémités, paree qu'à partir du petit axe, jusque vers les bouts, les bordśde la valve se ereusent en courbe concave et les deux demivalves forment un peu eomme deux fers de fièehe opposés par la base, qui est le stauros. Le raphé est double aussi vers le milieu des deux demi-valves, simple en approehant des nodules. La bande hyaline le long du raphé et le stauros médian, élargi sur les bords, ont le mème earaetère que dans l'espèee préeédente, mais les extrémités sont doublées à l'intérieur d'un épaississement qui les eoiffe d'un en-bout hyalin (tumen). Les stries sont délieates, formées de perles peu serrées, très peu rayonnantes et au nombre dè 12 environ dans 1 eent. de mm. Cette espèee, d'eau douee, longue de 8 à $15 ̋$ eent. 
de $\mathrm{mm}$., se trouve par groupes de 2 à 6 individus. La zone connective montre des plis portant des lignes perlées. Les frustules mesurent de 5 à 16 cent de $\mathrm{mm}$.

Le Stauroners gracilts, IV. Sm., est plus petit, sans épaississements aux extrémités, avec un stauros plus étroit, moins dilaté du còté des bords de la valve qu'il n’atteint pas le plus souvent. Les stries sont fines, perlées, assez rayonnantes. Le frustule ne mesure que de ä à 8 cent. de mm. Il vit dans les eaux douces, les tourbières, etc. (2, Fig. 224.)

Le Stauroneis spicula, W. J. Hickie, est très étroit, à bords presque droits, en losange très allongé, avec des extrémités pointues. Le raphé n'est double en aucun point, bordé d'une bande hyaline très étroite; le stauros est très étroitaussi, non dilaté aux bords de la valve. Les stries sont à peu près perpendiculaires au raphé, délicates, finement perlées et très serrées ( 28 dans 1 cent de $\mathrm{mm}$ ). C'est une espèce d'eaux saumàtres dont la taille est de 9 à 10 cent. de mm. (3, Fig. 224 ci-dessus.)

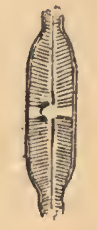

Fig. 226

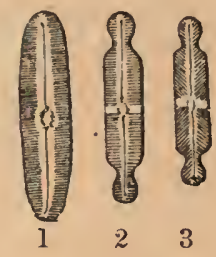

Fig. 227

Fig. 226. - Stauroneis anceps, Ehb.

Fig. 22\%. - 1. Nav. viridis, v. commutata, Gr.

2. Stauroneis anceps, var. amphicephala, $\mathrm{Kz}$.

3. Nav. bicapitata, Lag.

Quelques espèces ont les bords plus ou moins ondulés. Le Stauroneis anceps, Ehb., par exemple, est une jolie petite Diatomée d'eau douce à valves elliptiques avec les extrémités brusquement contractées, c'est-à-dire rostrées ou rostrées-capitées. Le stauros est relativement large, dilaté vers les bords de la valve, la bande hyaline le long du raphé très visible, les stries très rayonnantes, au nombre de 20 dans 1 cent de mill. Le frustule a de 4 à 6 cent de mm. de long.

Comme la plupart des espèces à capitations ou rostrations, celle-ci fournit diverses variétés dépendant de la forme et de l'amplitude des capitations. Telles sont les Stauroneis anceps var. linearis, $\mathbf{K z}$, et $\mathbf{S t}$. anceps var. amphicephala, $\mathbf{K z}$. Ces variétés ont les còtés parallèles, et, chez la première, les extrémités sont brusquement atténuées, mais non rostrées; dans la seconde, elles sont brusque- 
ment atténuées, mais rostrées (1). L'une et l'autre se trouvent mèlées au type dans les mèmes localités. Elles ont à peu près la mème taille. (2, Fig. 227.)

Les bords des valves peuvent ètre très ondulés. Tel est le Stauroneis legumen, Ehb, autre petite espèce d'eạu douce, de 3 cent. de $\mathrm{mm}$. de longueur, dont les cotés sont ondulés comme ceux d'une gousse qui renfermerait trois graines, celle du milieu plus petite. Les extrémités, rostrées, sont doublées d'un épaississement intérieur.

Le Stauroneis Smithii, Gr. n'en diffère que par son stauros un peu plus étroit et parce qu'il ressemble à une gousse dont la graine du milieu serait la plus grosse.

Nous signalerons encore plusieurs petites espèces d'eau douce, comme les Stauroneis pumila, Kz., le Stauroneis truncata, Schum, qui a 4 cent. de mm. de long. sur 1 1/2 de large; et le Stauroneis Cohnii, Hilse, jolie petite Diatomée ovale, de 3 cent. de $\mathrm{mm}$. environ de long sur $\mathbf{2}$ de large et qui présente $\mathbf{2 4}$ à 200 stries dans 1 centième de millimètre.

Quant au Stauroneis aspera, ou Navicula aspera d'Ehrenberg, il n'est pour nous un Stauroneis qu'en raison de son endochròme, dont les lames sont ondulées sur les bords, comme chez tous les Stauroneis, autrement nous le classerions parmi les Scoliopleura; mais il forme évidemment le passage de l'un à l'autre genre.

Nous avons déjà indiqué plus haut que nous considérions ce Navicula aspera comme une forme très aberrante et, à notre avis, égarée parmi les Navicula proprement dits dans lesquels il formait à lui tout seul un groupe à part, les groupes des Aspérées. (H. Van Heurck.) D'ailleurs, Ehrenberg et W. Smith, à l'époque où les genres étaient plus larges et leurs diagnoses moins serrées', avaient déjà été embarrassés pour classer.cette espèce, et ils en avaient fait tantôt un Stauroneis, tantôt un Stauroptera.

C'est une belle Diatomée longue, à côtés presque parallèles, avec des extrémités arrondies, des valves déprimées au centre, un raphé un peu flexueux partageant la valve à peu près par le milieu et bordé d'une bande hyaline qui s'étale, au niveau du nodule médian, en une zone lisse formant une ceinture transversale parfois complète, élargie sur les bords de la valve et au milieu de laquelle le nodule, grand et rond, est difficile à distinguer. Tout le restedes valves est cou-

(1) Les stries sont mal représentées dans la figure 227, 2 : elles doivent être rayonnantes et non perpendiculaires au raphé. 
vert de lignes rayonnantes composées de gros grains allongés. Ces grains font, en réalité, partie de stries interrompues et sont divisés en travers par des lignes difficiles à roir. Il y a 9 à 10 de ces stries

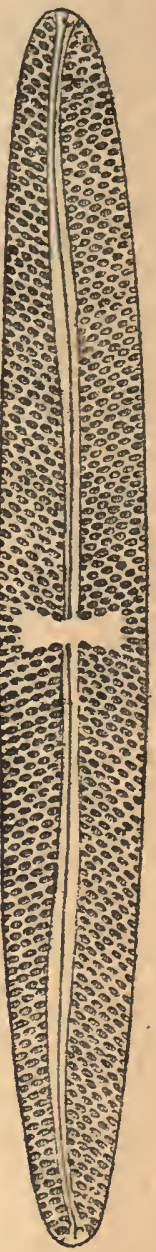

Fig. 228. - Stauroneis aspera, Ehb.

interrompues dans un cent. de mm. Le frustule, vu par la face connective, est contracté au milieu, épais et tronqué aux extrémités. Il a de 10 à 18 cent. de $\mathrm{mm}$. de long et sa valve est 6 à 8 fois plus longue que large. 


\section{\$. - Scoliopleura}

Le genre Scoliopleura a été créé par M. Grunow pour des Naviculées à frustules libres, à surface convexe, et un peu tordus en spirale de manière à rendre la zone connective et le raphé un peu sigmoïde.

Nous ne connaissons dans ce genre que deux espèces, marines: Scoliopleura latestriata, Gr., et Sc. tumida, Rab. Nous nous permettons d'en ajouter plusicurs, en donnant quelque extension à la caractéristique du genre telle qu'elle a été établie par M. Grunow.

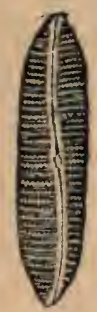

Fig. 229. - Scoliopleura latestriata, Gr.

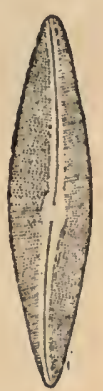

230. - Sc. tumida, Rab.

C'est ainsi que nous rapportons à ce genre Scoliopleura, tel que nous le définissons, plusieurs espèces appartenant à un genre Alloioneis, créé en 1867 par le naturaliste viennois J. Schumann(1), et qui n'a pas été conservé. Ce sont les Alloioneis Antillarum, Cl. et Gr., A. Kurzii, Gr., A. Grundleri, Cl. et Gr., et A. curvinervia, Gr. (2).

Dans ces conditions, nous pouvons définir ce genre en disant qu'il se compose de Naviculacées à frustules libres, parfois un peu tordus, à valves très convexes, marquées de stries perlées, s'alignant quelquefois en plusieurs systèmes, avec un raphé plus ou moins flexueux ou sigmoïde et ne divisant pas toujours la valve en deux parties symétriques.

Les Scoliopleura forment ainsi le passage des Navicula et autres Naviculacées symétriques, comme cclles que nous avons étudiées

(1) J. Schumann. Dic Diatomeen der hohen Tatra, 1867.

(2) P. T. Clève. Diatoms from the West Indian Archipelago, 1878. 
usqu'à présent, aux Naviculacées asymétriques ou sigmoïdes, comme la plupart de celles qui nous restent à examiner.

Ajoutons que toutes ces espèces sont marines, aussi bien celles dont nous avons parlé d'abord que les dernières.

Le Scoliopleura Antillarum, (Cl. et Gr.) Pell., a une forme elliptique lancéolée avec des extrémités subaiguës; la valve est un peu déprimée au centre et bombée près des extrémités, ce qui rend

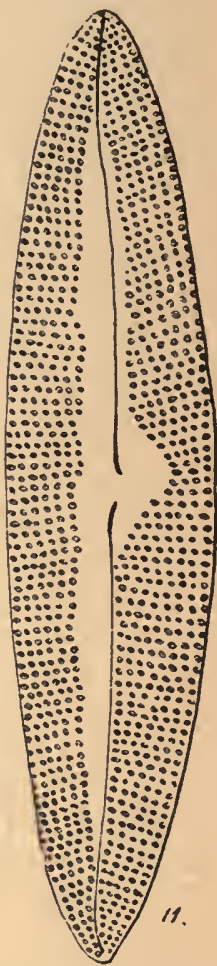

Fig. 231. - Scoliopleura Antillarum (Cl. et Gr.) Pell.

le raphé flexueux. Celui-ci est bordé sur une grande partie de sa longueur d'une bande hyaline très étroite ou même absente d'un côté, mais large de l'autre. Du côté où elle est le plus étroite, elle se dilate en une aire hyaline triangulaire qui embrasse le nodule médian, peu visible. Les stries sont rayonnantes, formées de gros grains interrompus, mais nous ignorons si ces grains sont divisibles en parties plus fines. Ces stries, du còté du raphé, sont d'inégales longueurs. Le frustule a 12 cent. de mm. de longueur (Grunow). C'est 'Alloioneis Antillarum de Clève et Grunow. 
Le Scolioplewra tumiăa, Rab, a une forme elliptique lancéolée, avec des extrémités ordinairement assez aiguës. La valve est déprimée au centre, bombée vers les extrémités, ce qui rend le raphé flexueux. Il est bordé d'une mince bande hyaline qui se dilate en ellipse à grand axe longitudinal autour du nodule médian, difficile à roir. Les stries, au nombre de 10 environ dans 1 cent. de mm., rayonnantes au milieu, un peu courbes à cause de la forme de la valve,
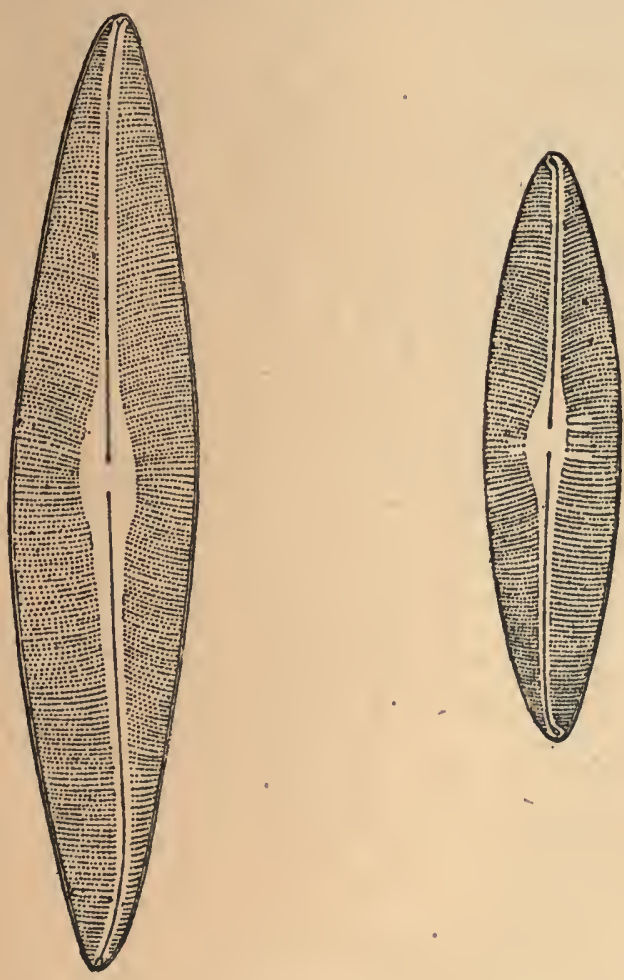

Fig. 232. - Scoliopleura tumida, Rab.

sont continues, mais finement perlées. Elles sont souvent de longueur inégale dans la région médiane. Le frustule mesure de 10 à 16 cent. de $\mathrm{mm}$. de longueur.

Le Scoliopleura Kurzii (Gr.) Pell., est l'Alloioneis Kurzui de Grunow. Il a des frustules elliptiques larges à extrémités atténuées. Sa longueur est environ trois fois sa largeur. Le raphé est flexueux et ne partage pas la valve en deux parties égales; il est bordé d'une bande hyaline plus étroite d'un côté que de l'autre et brusquement 
dilatée du còté plus étroit autour du nodule médian, en raison de ce que les stries médianes restent très courtes de ce cóté et à ce niveau. De l'autre cóté, les stries, formées partout d'assez grosses granulations, paraissent laisser le long du raphé et autour du nodule médian une surface lisse plus large, mais ce n'est qu'une appa-

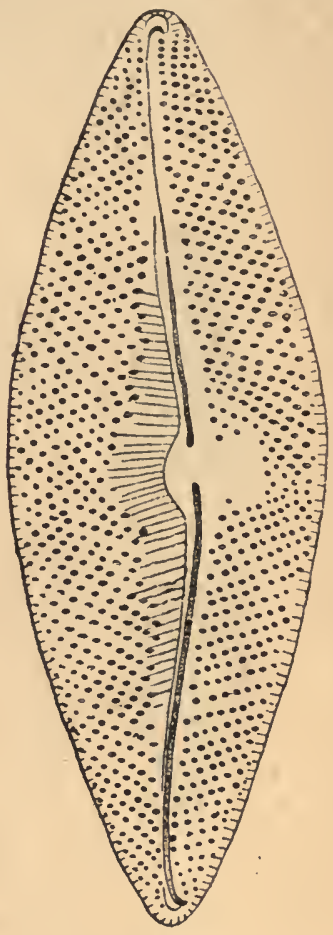

Fig. 233.
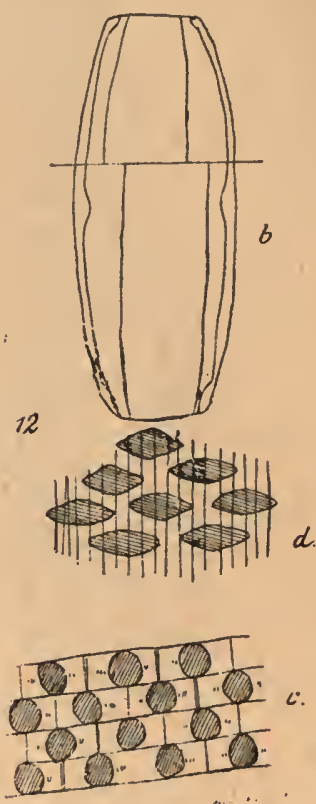

Fig. 231.

Fig. 233. - Scoliopleura Lursii (Gr.) Pell.

Fig. 234. - Scoliopleura Kurrzii. Détails :

b. Frustule entier (montrant lapartie sup. et la partie inférieure).

c. Perles décussées, lumière centrale; gross. 3040 diamètres.

$d$. Les mèmes, éclairage oblique; gross. 3040 diam. (P. T. Clève).

rence : les stries se continuent de ce côté jusqu'au raphé et presque jusqu'au nodule médian, seulement elles ne sont plus ponctuées et passent, dans cette partie, à l'état de côtes. Quant aux stries ponctuées elles-mêmes, elles sont disposées de manière à s'aligner en trois systèmes (ou décussées), un système transversal et deux systèmes obliques qui se croisent. Les stries transversales sont plus 
serrées que les stries obliques et au nombre de 10 à 11 dans 1 cent. de $\mathrm{mm}$. (Grunow.) Le frustule mesure de 9 a 10 cent. de $\mathrm{mm}$.

Le Scoliopleura Grundleri (Cl. et Gr.) Pell., est une espèce un peu plus petite, a frustule bacillaire par sa face valvaire, avec les côtés parallèles et les extrémités presque arrondies mousses. Il est environ quatre fois plus long que large et parait un peu déprimé au milieu. Le raphé est flexueux et partage la valve en deux parties

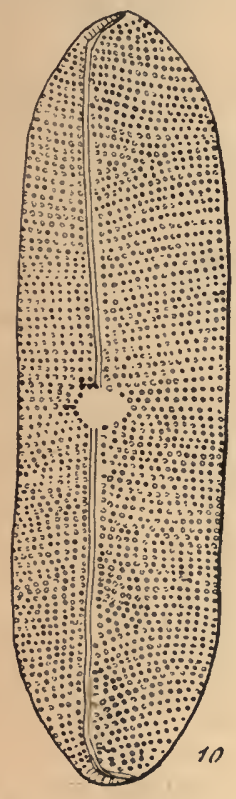

Fig. 235. - Scoliopleura Grundleri (Cl. et Gr.) Pell.

très inégales. Les stries sont transversales, atteignant le raphé des deux côtés, mais elles laissent un petit espace lisse, en ellipse transversale, autour du nodule médian. Elles sont composées d'assez gros grains et au nombre de 11 dans 1 cent. de $\mathrm{mm}$. Le frustule n'a que $71 / 2$ cent. de $\mathrm{mm}$. de long.

Le Scoliopleura curvinervia (Gr.) Pell., a une forme elliptique lancéolée, régulièrement atténuée du milieu aux extrémités. Le frustule est quatre fois aussi long que large et parait gonflé au centre; le raphé est flexueux et ne partage pas la valve en deux parties tout à fait égales; il est bordé par une bande hyaline qui va en s'élargissant des extrémités vers le milieu, où elle se dilate autour du nodule médian, plus d'un côté que de l'autre. Les stries sont rayonnantes et ont l'aspect de còtes, mais avec un fort grossis- 
sement, on reconnait qu'elles sont formées de perles très fines et difficiles à voir. Il y a de 8 à 9 stries dans 1 cent de mm. (Grunow) et elles sont interrompues par un sillon très net, à peu près parallèle au bord de la valve, qui les coupe de chaque còté du raphé, à près de la moitié de leur longueur. Le frustule mesure de 7 à 11 1/2 cent. de mm. (Fig. 236.)

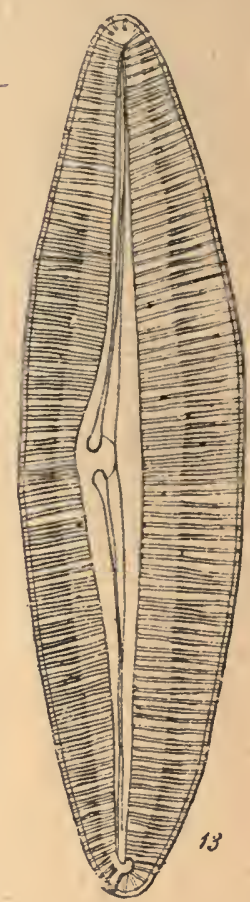

Fig. 236. - Scoliopleura curvinervia (Gr.) Pell.

Le Scoliopleura latestriata, Gr., a une forme bacillaire longue avec les côtés parallèles et les extrémités peu à peu atténuées. Le frustule, environ six fois aussi long que large, parait un peu bombé vers l'une de ses extrémités et un peu déprimé vers l'autre, ce qui donne au raphé une forme non seulement flexueuse, mais sigmoïde. Celui-ci est bordé d'une très étroite bande lisse, qui s'élargit légèrement autour du nodule médian. Les stries se présentent sous forme de còtes robustes, 7 a 8 dans 1 cent. de mm., mais entre ces còtes on reconnait, avec un bon objectif, deux rangées de perles alternées, très fines et difficiles à voir; ce qui, avec le système transversal, constitue trois systèmes croisés. Ces stries sont interrompues de chaque côté, dans le voisinage du raphé par un sillon 
très marqué parallèle à ce raphé. Cette Diatomée a de 10 à 1 č cent. de $\mathrm{mm}$. de longueur.

Telles sont les espèces à nous connues qui nous paraissent devoir ètre rapportées dans un mème genre, lequel à notre avis doit plutòt être le genre Scoliopleurce que tout autre. Nous avons peut-ètre insisté un peu trop longuement sur les quelques formes que nous y

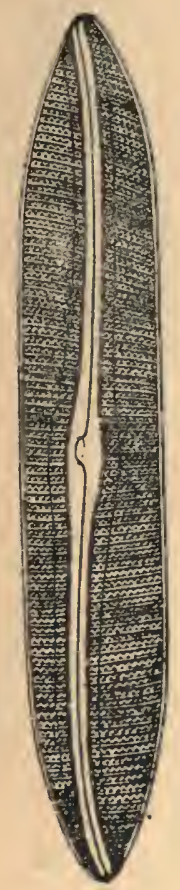

Fig 237. - Sc, latestriäta, Gr.

réunissons et dont quatre sur six sont rares et appartiennent aux mers de l'Inde ou du Mexique; mais nous tenions à faire ressortir les points de contact, nombreux et variés, qui les rapprochent naturellement et nous paraissent justifier le remaniement que nous avons fait subir à ce genre.

Nous ferons encore remarquer à ce sujet que la position systématique de ces espèces est bien où nous l'établissons, car nous les royons par leur raphé flexueux, quelquefois décidément sigmoïde, et mème par certains détails de leur striation, offrir un passage naturel aux Pleurosigma, qui les avoisinent en effet dans le tableau de classification des Naviculacées. De plus, les lames d'endochròme, du moius dans les espèces où elles ont été observées, présentent 
un lobe saillant vers le nodule médian, conme chez les Rleurosigma. Et mème, nous y voyons apparaitre l'approche de la famille suivante, celle des AMPHIPRoRÉEs, dans les espèces dont les valves sont bombées vers leurs extrémités, et particulièrement dans le Scoliopleura latestriata, dont la valve est bombée dans une de ses moitiés et déprimée dans l'autre. Cette disposition est, d'aillcurs, bien

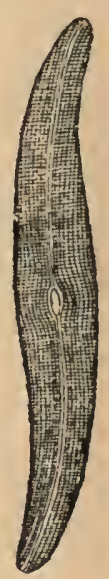

Fig. 238. - Pleurosigma hippocampus, Ëhb. (stries rectangulaires).

plus accentuée dans une forme qui appartient aussi aux mers des Antilles, le' Rhoïcosigma Antillarum, Cl. et Gr., dont nous parlerons plus loin.

\section{$\S 3-$ Pleurosigma}

Le genre Pleurosigma, créé par W. Smith, est un des plus beaux de la famille des Diatomées. Ses caractères nettement tranchès, et qui permettent de distinguer à première vue les espèces qui le composent de celles de tous les genres voisins, en font un des groupes les plus naturels que les diatomistes aient établis. Ces espèces sont souvent de très grande taille, très élégantes de forme et marquées d'admirables systèmes de stries, qui depuis fort longtemps ont fait de quelques-unes des tests très pratiques, très usités et très utiles.

Les frustules sont naviculaires; en général allongés, mais plus ou moins tordus en $\mathbf{S}$ quand on les regarde par la face valvaire. 
Le raphé suit naturellement cette eourbe, mais il est souvent plus sigmoïde, d'autres fois moins, que la valve elle-mème. Ces valves ne sont ordinairement pas planes, mais un peu bombées dans le sens transversal. Les frustules vus par la face eonneetive sont droits.

M. Otto Müller a étudié d'une manière spéciale l'endoehròme de certains Pleurosigma et a trouvé qu'il est formé, eomme dans toutes

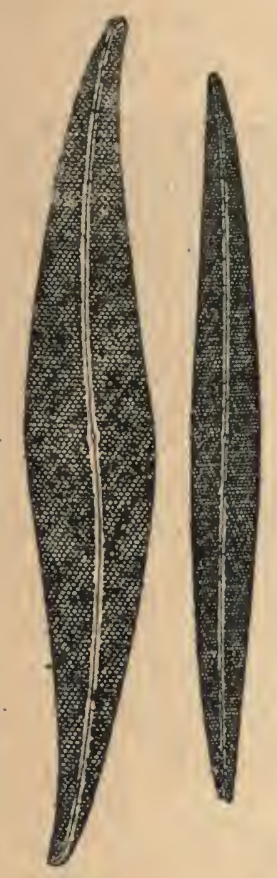

Fig. 239.

Fig. 240.

Fig. 239. - Pleurosigma angulatum, W. Sm. (1)

Fig. 240. - Pleurosigma intermedium, W. Sm.

(Stries décussées).

les Naviculées, de deux plaques ou ehromatophores qui reposent par leur ligne médiane sur la ligne médiane de la zone connective, mais leurs bords sont déehiquetés, avee un lobe qui s'avanee ver's le nodule médian, et leur surface présenterait des perforations régulièrement disposées.

(1) Dans les figures que nous donnons des Pleurosigma, on comprend que nous n'avons pas pu représenter les stries à leur véritable distance. Elles sont, en géneral, deux fois plus serrées sur les valves naturelles que dans nos dessins, excepté dans les reproductions d'épreuves photographiques. 
Les Pleurosigma se trourent le plus ordinairement à l'état de frustules libres; cependant il parait qu'on les rencontre quelquefois dans des tubes gélatineux.

Les stries, si remarquables, qui ornent leurs valves peuvent ètre disposées de deux manières. Elles sont composées de perles, qui s'alignent souvent en deux systèmes perpendiculaires l'un à l'autre. Les stries sont alor's dites "rectangulaires». Mais, d'autres fois, les perles sont disposées en quinconce et s'alignent en trois directions, une transversale et deux obliques, qui se croisent sous des

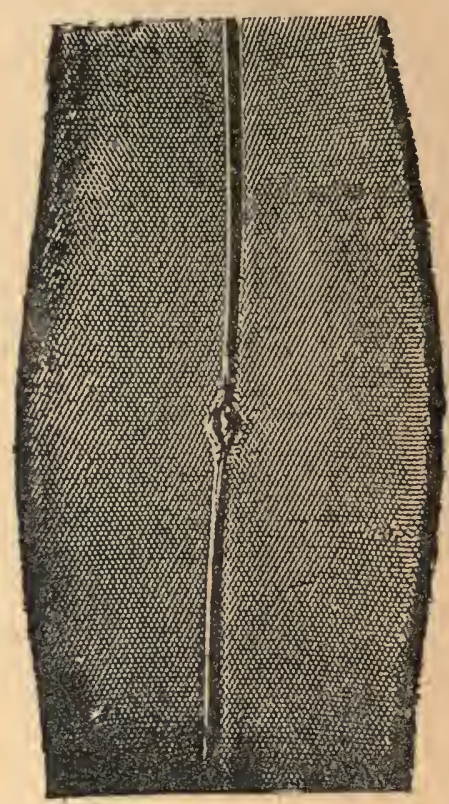

Fig. 241. - Pleurosigma angulatum, résolu et photographié à la lumière électrique par le $\mathrm{D}^{\mathrm{r}} \mathrm{H}$. Van Heurck.

angles un peu variables, l'une de lignes obliques étant dirigée de droite à gauche et l'autre de gauche à droite. Dans ce cas, les stries sont dites «décussées ». Suivant le mode d'éclairage que l'on emploie et la direction de la lumière oblique que l'on dirige sur la valve, on peut faire apparaitre à volonté le systéme des stries transversales, l'un ou l'autre des systèmes obliques et mème parfois un quatrième systéme, longitudinal, comme en le comprend en examinant le schéma que nous en avons donné. (Voir Fig. 52, p. 73.)

Résolues en perles, comme on dit, les stries paraissent formées par des grains hexagonaux, mais M. Alfred Nachet a fait voir jadis qu'il s'agit là d'une illusion d'optique produite par l'entrecroise- 
ment des trois systèmes d'interstries et que les grains sont arrondïs. C'est ce qu'il est facile aujourd'lui de vérifier avec les objectifs que nous possèdons (1).

Cette dirersité dans la disposition des stries permet de diviser d'une manière très simple les Pleurosigma en deux groupes : ceux qui présentent des stries décussées et ceux qui ont des stries rectangulaires.

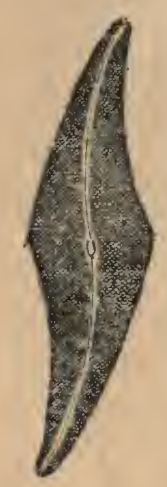

Fig 212.

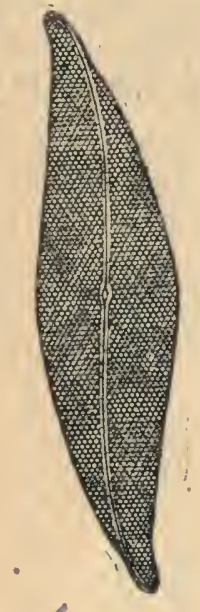

Fig. 243.

Fig. 242. - Pleurosigma angulatum var. quadratum, W. Sm.

Fig. 243. - Pleurosigma angulatum var. Estuarii. W. Sm.

Parmi les espèces à stries décussées, la plus remarquable de toutes est le fameux Pleurosigma angulatum, W. Sm., qui sert de test, depuis bien des années, pour les objectifs moyens. Il a une forme lancéolée un peu élargie au milieu, avec un raphé dont la courbure suit à peu près celle de la valve. Ces stries, au nombre de 18 à 20 dans 1 cent. de millim., ont la mème force dans tous les sens et sont orientées dans les mèmes directions sur toute la valve. Les stries obliques sont un peu moins serrées que les stries transversales. Woodward a obtenu autrefois de magnifiques photographies de ce Pleurosigma résolu en perles, à la lumière solaire monochromatisće, avec les objectifs de Prazmowsky et de Powell et Lealand, et le $\mathrm{D}^{\mathrm{r}} \mathrm{H}$. Van Heurck a donné aussi récemment d'admirables épreuves réalisées à l'aide de l'éclairage électrique.

Cette belle espèce marine peut atteindre jusqu'à $10 ّ$ centiẻmes de

(2) Voir J. Pelletar, Le Microscope, son emploi et son application, p. 577. 
millimètre de longueur. Elle fournit un assez grand nomore ae jolics variétés, dont les plus importantes sont les suivantes :

D'abord, une forme plus grande, qui peut attcindre 22 centièmes, le $P l$. angulatum, forma major, et que beaucoup de diatomistes considèrent comme le type, désignant alors celle que nous venons de décrire sous le nom de $P l$. angulatum, forma minor.

Le $P l$. angulatum var. quadialum est beaucoup plus large, a une forme losangique anguleuse tordue, un raphé plus flexueux. Il y a des espèces qui diffèrent moins l'une de l'autre que cette " variẻté » ne diffère du type.

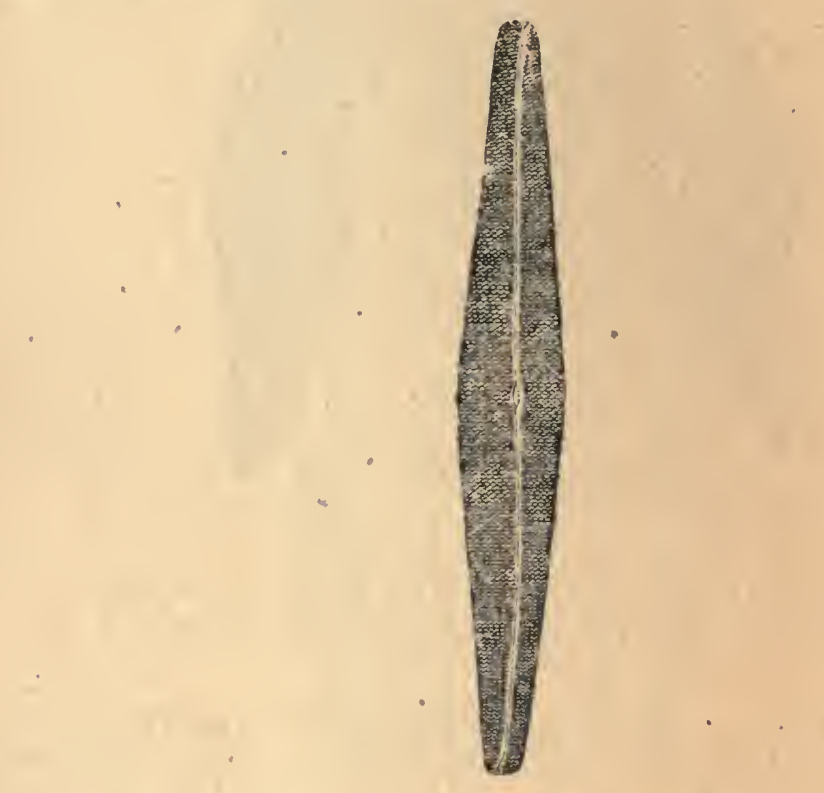

Fig. 244. - Pleurosigma rigidum, W. Sm.

Le Pleurosigma angulatum var. Astuarii, W. Sm. sc rapproche davantage du type, quoique plus petit, plus large, avec des extrémités un peu rostrées et un raphé plus sigmoïde que la valve elle-même, aussi devient-il tangent à l'un des bords vers chacune des extrémités.

M. H. Van Heurck donne encore comme variétés du type $P l$. angulatuni, le $P l$. delicatulum, W. Sm., très long et très étroit, à stries un peu plus serrées, moins atténué aux extrémités; l'énorme $\mathrm{Pl}$. strigosum, qui peut atteindre $1 / 3$ de millimètre de long, non anguleux, et obtus; le $P l$. elongatum, très long, très mince et dont les strieș se croisent sous un angle plus aigu. 
Le Pleurosigina affine, Gr. est une espèce voisine, lancéolée large, à peine tordue, mais dont le raphé est plus sigmoïde que la

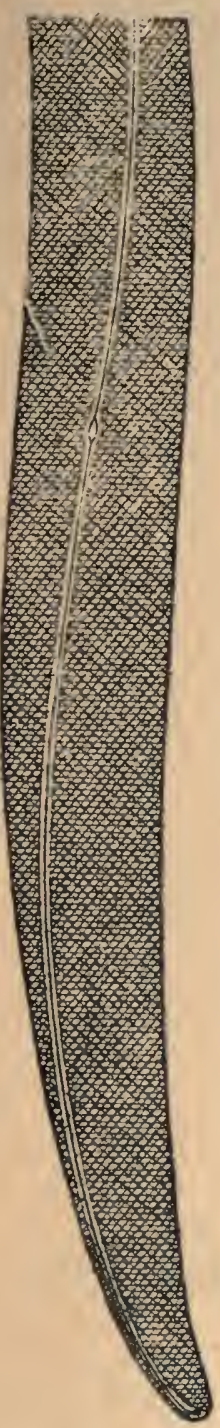

Fig. 245.

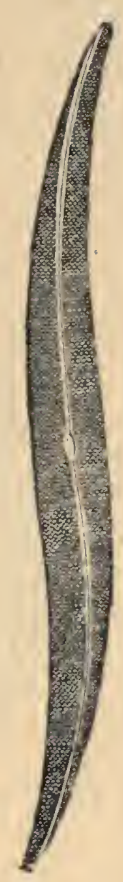

Fig. 246.

Fig. 245. - Pleurosigma formosum, W. Sm.

Fig. 246. - Pleurosigma decorum, W. Sm.

valve. Les stries obliques se coupent à angle droit, vers le milieu de la valve, où elles sont flexueuses, et à angle aigu aux extrémités, 
18 à 20 dans 1 cent. de mm. C'est encore une espèce marine qui peut atteindre 20 à 22 cent. de mill.

Le Pleurosigma intermedirm, W. Sm., est très peu courbé, très étroit et très long, avec un raphé presque droit. Les stries se coupent partout sous le mème angle et les stries transversales sont plus serrées (20 à 24 dans 1 cent. de $\mathrm{mm}$ ). Le frustule mesure de 13 à 30 cent. de mm, et les plus petits individus sont les plus sigmoïdes. (Grunow.) Espèce marine. (Fig. 240.)

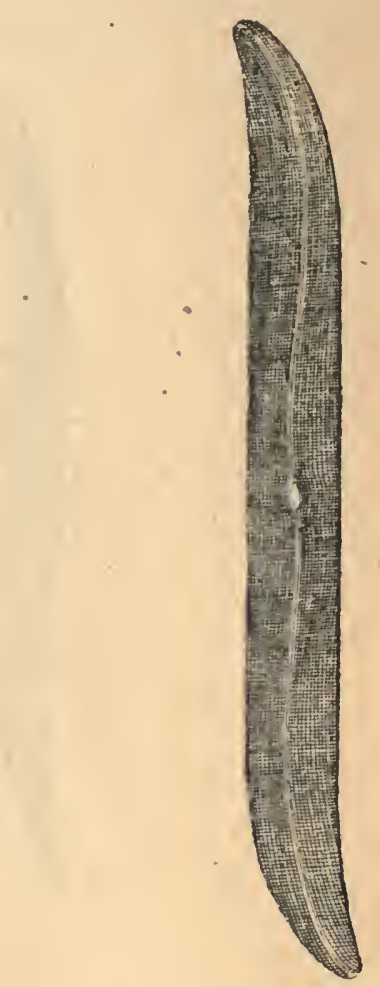

Fig. 247. - Pleurosigma balticum, W. Sm.

Le Pleurosigma rigidum, W. Sm., se distingue tout de suite à sa forme, qui est droite, le raphé seul est très légèrement sigmoïde. Les extrémités sont brusquement obtuses. Il a des stries comparables à celles des espèces précédentes; c'est aussi une espèce marine, longue de 13 a 26 cent de mm. (Fig. 221.)

Nous citerons encore les deux espèces suivantes, dont le raphé est beaucoup plus tordu que les valves et, par conséquent, coupe celles-ci en deux parties très inégales vers les extrémités. Les stries 
obliques sont plus fortes et moins serrées que les stries transversales qui sont assez fines :

Pleurosigma formosum, énorme espèce maṛine qui atteint 1/2 millimètre dans les forts individus, et dont les stries obliques se coupent à angle droit. Il y a $1 \ddot{\text { à }} 17$ stries transversales fines et 10 à 12 obliques dans 1 cent. de millimêtre. (Fig. 243.)

Pleurosigma decorum, WV. Sm., autre grande espèce marine, de 20 à $20 ̈$ centièmes de millimètre, étroite, longue, pointue, très sigmoïde, à raphé plus flexueux que la valve, et dont les stries obliques, fortes, se coupent presque (?) à angle droit, au nombre de 14 environ dans 1 centième de millimètre, tandis que les stries transversales, fines, sont au nombre de 18. (Fig. 246.)

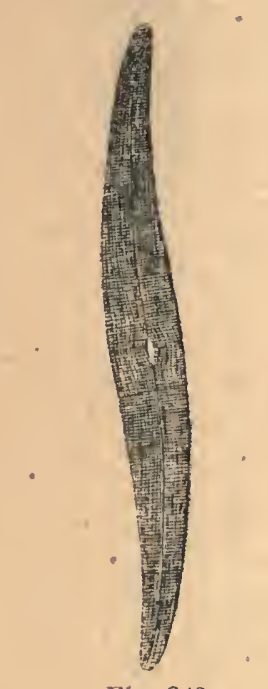

Fig. 24s.

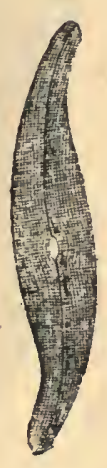

Fig. 249.

Fig. 248. - Pleurosigma acuminatum, Grun.

Fig. 249. - Pleurosigma scalprum, Gr.

Les espèces qui n'ont que deux sysièmes de stries, rectangulaires, c'est-à-dire des stries transversales et des stries longitudinales perpendiculaires les unes aux autres, sont assez nombreușes. Les plus remarquables sont les suivantes :

Le Pleurosigma ballicum, W. Sm., est une grande espèce marine, longue de 20 à 36 centièmes de millimètre, moyennement large et dont les longs côtés sont drỏits et parallèles, la courbure ne se produisant qu'aux extrémités. Le raphé, est.ordinairement 
plus sigmoïde que la valve et flexueux au voisinage du nodule médian. Les stries longitudinales s'incurvent un peu autour de ce nodule et elles sont à pcu près aussi scrrées quc lcs stries transversales, environ 1 s dans 1 cent. de millim. (Fig. 247.)

Le Pleurosigma scalprum, Gr., est une espèce d'eau saumàtrc ou salée, à valves assez larges, avec les extrémités atténuées et recourbées, le rcste de la valve restant sans flexion. Les stries longitudinales et transversales sont de mème valeur, comme dans l'espèce précédente, mais plus délicates. Le frustule n'a que 10 à 180 cent. de mm. (Fig. 249.)

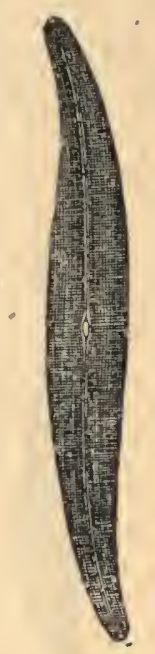

Fig. 250.

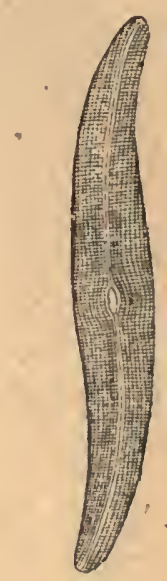

Fig. 251.

Fig. 250. - Pleurosigma attenuatum, W. Sm.

Fig. 251. - Pleurosigma hippocampus, IV. Sm.

Le Pleurosigma acuminatum, Gr., est une espèce d'eau douce, assez commune, ayant de 13 à 17 ccntièmes dc millimètre, assez sigmoïde, avec un raphé qui partage la valve en deux parties presque égales. Il est un peu renflé au milieu avec des extrémités légèrement acuminées. Les stries longitudinales et transversales sont également marquées et serrées, 18 environ dans 1 cent. de mill. (Fig. 248.)

Dans le Pleurosigma attenuatum, W. Sm., les striés longitudinales sont dominantes, sans doute parce qu'elles sont moins serrées; il n'y en a, en effet, que 10 environ dans 1 centième de mm., tandis qu'il y a 14 à 16 stries transversales. Elles s'incurvent un peu con- 
centriquement au nodule médian. Le frustule est plus grêle que dans l'espèce précédente, moins large et s'atténue insensiblement depuis le milieu jusqu'aux extrémités. Le raphé partage la valve en deux parties sensiblement égales. C'est encore une espèce d'eau douce, longue de $1 / \check{s}$ à $1 / 4$ de millimètre.

Le Pleurosigma hippocampus, W. Sm., ressemble beaucoup au précédent pour le nombre et la disposition des stries, dont le systeme longitudinal domine; mais il est plus trapu, plus court et plus large, sa longueur ne dépasse pas 16 centièmes de millim. Il vit dans les eaux saumàtres, à l'embouclıure des fleuves.

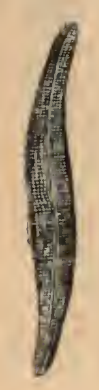

Fig. 252.

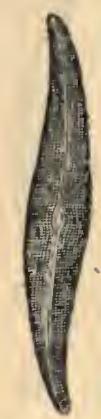

Fig. 253.

Fig. 252. - Pleurosigma curvuIn, Gr.

Fig. 253. - Pleurosigma Kutzingii, Gr.

Le Pleurosignia curvulum, Gr., est une petite forme d'eau saumâtre, longue de 8 à 12 centièmes de mm., assez grèle, neuf à. dix fois plus longue que large, sigmoïde, et dont les stries longitudinales sont plus serrées que les stries transversales, contrairement à ce qui a lieu dans les espèces précédentes. Il y a 24 à 200 stries longitudinales et 21 à 22 transversales dans 1 cent. de $\mathrm{mm}$.

Le Pleurosigma Kü̈zingii, Gr., d'eau douce, est un peu plus grand, car il peut atteindre 14 centièmes de mill.; il est aussi relativement plus large, avec les extrémités plus atténuées. Sa largeur, en effet, n'est guère que six à sept fois plus petite que sa longueur. Les stries longitudinales sont un peu plus serrées que les transversales : $221 / 2$ des premières pour $201 / 2$ des secondes dans 1 cent. de $\mathrm{mm}$.

Enfin, le Pleurosigma nodiferum, Gr., ressemble par sa forme générale au $\mathrm{Pl}$.hippocampus, c'est-à-dire qu'il est presque droit, avec les extrémités seulement infléchies; mais il est plus petit, 
n'ayant que de 8 à 12 eent. de mm. de longueur el environ 1 à $11 / 2$ de largeur. Son raphé est assez sigmoïde et il présente la mème disposition que les précédents pour les stries, dont le système longitudinal est plus serré (22 à 23 stries pour 1 eent. de mm.) que le système transversal (17 à 20 stries). Il offre cette particularité intéressante qu'il y a un très petit espaee hyalin eoupant obliquement le nodule médian, el que les stries transversales médianes sont un peu rayonnantes autour de ce nodule. Il vit dans l'eau douee.

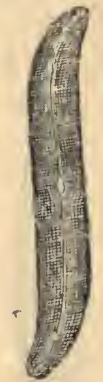

Fig. 254.

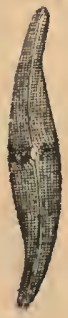

Fig. 255.

Fig. 254. - Pleurosigma nodiferum, Gr.

Fig. 255. - Pleurosigna Parkeri, Harr.

Le $\mathrm{D}^{\mathrm{r}} \mathrm{H}$. Van Heurck réunit ees trois dernières formes : Pleurosigma curvulun, $\mathrm{Pl}$. Kützingii, $\mathrm{Pl}$. nodiferum et quelques autres voisines au Pleurosigma Spencerii, W. Sm., espèee d'ean douce, dont elles ne seraient que des variétés.

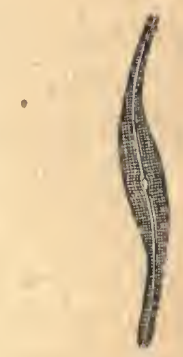

Fig. 256. - Pleurosigma fascîola, Ehb.

Mais le Pleurosigma Parkeri, Harr., présente une disposition particulière des stries médianes, plus remarquable encore que eelles dont le $P l$. nocliferm, Gr., nous a donné un exemple, disposition caraetéristique. Iei, ce sont les stries Iongitudinales qui, à la hauteur du nodule médian, déerivent de petites courles qui se eoupent en formant au frustule une ceinture de petites figures elliptiques. 
C'est, d'ailleurs, une petite espèee d'cau douee, de 8 à 10 eent. de $\mathrm{mm}$. de long, assez large, à extrémités un peu brusquement atténuées et fléehies, avee un raphé qui divise la valve en parties inégales. (Fig. 2̈̈̈.)

Quant au Pleurosigma fasciola, Ehb., il présente, exagérée, l'atténuation brusque des extrémités, qui se prolongent en deux rostres minees et fléehis en sens contraire. Le raphé partage la valve en deux parties égales et les stries sont disposées comme dans les espèees préeédentes, e'est-à-dire que ec Pleurosigma appartient au groupe dont les stries longitudinales sont plus serrées que les stries transversales, eomme 23 cst à $\mathbf{2 1}$, dans 1 cent. de $\mathbf{m m}$. C'est une espèec marine, longue de 10 eent. de $\mathrm{mm}$. et très caraetérisée.

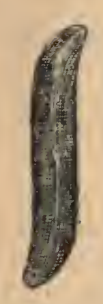

Fig. $25 \%$.

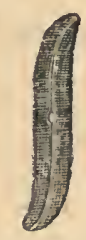

Fig. 25s.

Hig. 257. - Rleurosigma scalproides, Rab.

Fig. 258. - Pl. eximium, |Thw:

Les Pleurosigma scalproïdes, Rab., et $P l$. eximium, Thw., sont deux autres petites espèees, longues de 8 à 10 eent. de mm., appartenant eneore à ee groupe par la disposition des stries longitudinales plus serrées. Ils ont la forme épaisse, à eorps droit, avee les eôtés parallèles et les extrémités scules fléehies, en lame de sealpel. Le $P l$. scalproüdes habite les eaux douees; il a 6 à 7 eent. de $\mathrm{mm}$. de long et les stries longitudinales sont aux transversảles eomme 29 est à 22, dans 1 ecnt. de mm. Le Pl. eximinem, qui cst marin, ou d'eau saumàtre (embouehures), n'a que $301 / 2$ cent. de $\mathrm{mm}$. avec 28 stries longitudinales pour 24 transversales dans 1 cent. de $\mathrm{mm}$.

Ces deux dernières espèees sont remarquables par ee détail partieulier que leurs frustules se forment dans des tubes gélatineux, eomme les Schizonema. Aussi, le Plenrosigma eximium de II. Van Ileurek avait-il reçu de Brébisson le nom générique d'Endosigma eximium, le préfixe Endo rappelantl'intérieur des tubes. D'autre part, Ehrenberg en avait fait un Gloionema sigmoüdeum, indiquant ainsi le filanent glaireux formé par le tube et la forme du frustule. Thwaites en fit tout simplement un Schizonema exi- 
mium et Sullivant le plaça avec raison dans les Pleurosigma, mais sous le nom de $P l$. obtusatum. C'est le $D^{r}$ II. Van Heurck qui a rectifie cette désignation en Pleurosigma eximium.

Telles sont les principales espèces et variétés de Pleurosigma; nous devons ajouter que les unes et les autres sont sujettes à d'innombrables variations dans la forme et la taille, mais la disposition des stries ne change pas, et leur nombre dans un espace donné ne varie que dans des limites très étroites et qui ne suffisent pas à modifier l'aspect de la striation.

En résumé, on voit que ce beau genre, dont les espèces passent presque insensiblement de l'une à l'antre, se divise naturellement en deux grands groupes :

$1^{\circ}$ Espèces à trois systèmes de stries (décussées);

$2^{\circ}$ Espèces à deux systèmes de ștries (rectangulaires).

Le premier groupe se subdivise en deux types :

A. Type à stries toutes pareilles, se croisant sous un angle aigu, plus ou moins voisin de $60^{\circ}$. Les espèces se distinguent alors par la forme : $P l$. angulatum, $P l$. quadratum, $P l$. costuarii, $P l$. intermedium, $\mathrm{Pl}$. rigidum, etc.

B. Type à stries obliques se croisant à angle droit. On distingue les espèces par la forme: Pleurosigma formosum, Pl. decorum.

Les espèces de ce type à trois systèmes de stries, dont deux perpendiculaires ou rectangulaires, forment la transition aux espèces du second groupe.

Ce second groupe comprend, en effet, les espèces à deux systèmes 'de stries, rectangulaires. Il se divise à son tour en trois types.'

C. Type à stries également marquées ou distantes dans les deux sens; on distingue les espèces à la forme, (Pl. balticum, $\mathrm{Pl}$. acuminatum, $P l$. scalprum, etc.).

D. Type à stries longitudinales dominanteś, parce qu'elles sont moins serrées. Les espèces se reconnaissent à la forme ( $P l$. attenuatum, $\mathrm{Pl}$. hippocampus, etc.).

E. Type où les stries transversales sont dominantes parce qu'elle $\mathbf{S}$ sont moins serrées. Les espèces se distinguent à leur forme, à diverses particularités caractéristiques de la valve ou à leur formation dans des tubes (Pl. Spencerii, Pl. curvulum, Pl. Küt:ingii, $P l$. fasciola; $P l$. nodiferum, $P l$. Parkeri; Pl. eximinm, Pl. scalprö̈des, ctc). 


\section{$\S 4$. - Donkinia, Toxonidea, etc.}

C'est ici que devrait se placer la deseription de diverses formes de transition qui relient les Pleurosigma aux familles voisines; malleureusement, le plus grand nombre sont assez peu connues, exotiques d'ailleurs, et très rares. Aussi, nous citerons seulement pour mémoire les deux suivantes.

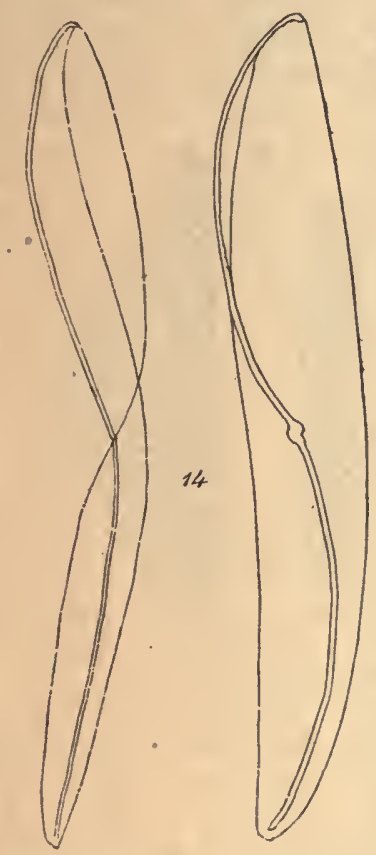

Fig. 259. - Rhoïcosigma Antillarum, $\mathrm{Cl}$.

Le Rhoïcosigma Antillarum, Cl., que nous avons déjà signalé à propos des Scoliopleura, est une espèce à forme pleurosigmée dont le raphé, très sigmoide, forme une erête très saillante dans une moitiẻ de la valve et plonge, au eontraire, en.dépression profonde dans l'autre moitié. La valve est distinctement striée' transversalement, de 14 à 13 stries dans 1 eent. de mm. Le frustule n'a que $41 / 4$ eent. de mm. Nous n'avons pas d'autres détails. Du reste, eette espèce n'est pas unique, et M. Grunow en a décrit, plusieurs autres. Ces formes se rapprochent beauconp des Donkinia, si même on ne doit 
les classer dans ce genre, qui fait une transition très marquée aux Aypiniprorḱes, ou dans les Aypirprorées elles-mèmes.

Les Donkinia, constituent 'un petit genre créé par Ralfs, en 1860, et qui contient quelques éspèces chez lesquelles le raphé forme une crète saillante ou carêne, sigmoïde, au milieu de laquelle est le nodule médian, et le frustule vu par la face connective est fortement contracté au milieu.

Tel est le Donkinia recta, Gr., dont le frustule vu par la face valvaire est droit, à còtés parallèles, à extrémités en lancette obtuse, traversé en écliarpe par un raphé saillant. La valve est couverte de stries, sans aires lisses, à deux systèmes, rectangulaires, 21 stries environ dans 1 cent. de mm. L'espèce, marine, mesure 8 à 9 cent. de. $m \dot{m}$.

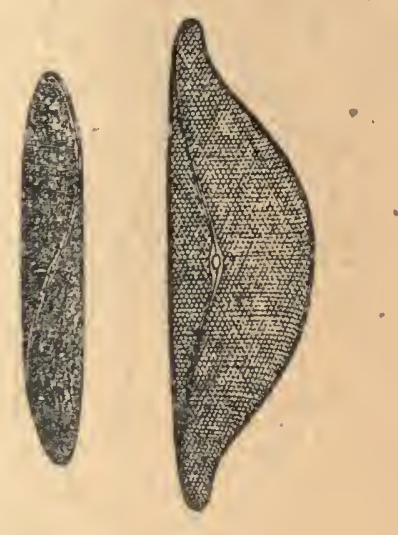

Fig. 260. Fig. 261.

Fig. 260. - Donkinia recta, Gr. Fig. 261. - Toxonidea insignis, Donk.

C'est un Pleurosigma droit, à raphé saillant et formant doux crêtes ou carènes, l'une dans la demi-valve supérieure, l'autre dans la demi-valve inférieure, avec un'nodule médian déprimé. Donkin, qui l'a découvert, l'avait nommè Pleurosigna rectum.

Quant aux Toxonidea, Donkin, ils représentent encore des Pleurosigma déprimés, Ainsi le Toxonidea insignis, Donkin, est un Pleurosigma droit qui a une énorme fluxion sur un còté. Il en résulte une Diatomée asymétrique à raphé courbe, partant du bord droit et y rerenant en passant par un nodule médian excentrique. Les extrémités se sont rostrées par suite de la tuméfaction unilatérale du frustule. Les stries sont restées celles d'un Pleurosigma à trois systèmes (décussées), 21 environ dans 1 cont. de mm. C'est 
une espèce marine, rare, mais que l'on trouve, comme la précédente, sur les moules et qui a tout à fait l'aspect d'un Epithemia. Ce scrait une forme de passage aux Cynbeluées.

Une autre forme de transition a été trourée par M. L. Reinhard, naturalistc russe, dans des récoltes faites par.M. Mereschkowsky dans

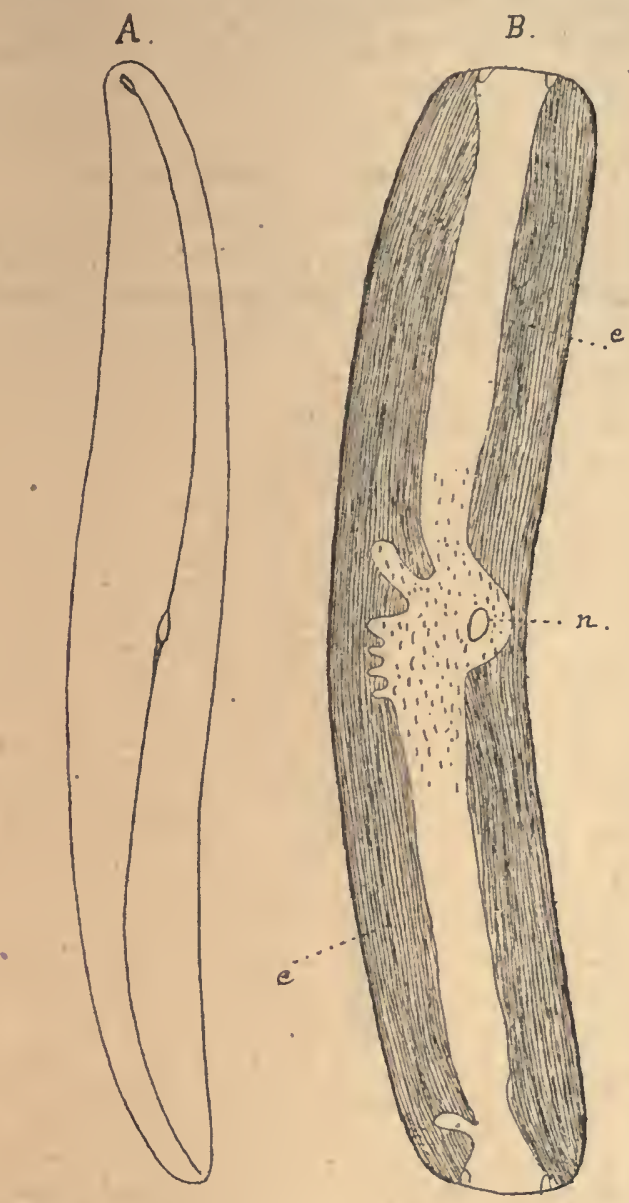

Fig. 262. - Achnanthosigma Mereschouskii, Reinh.

A. face valvaire; $B$, face connective; $c$, endochrôme, $n$ noyau.

la mer Blanche. Sous le nom d'Achnanthosigma Mereschkowshii, il en a fait le type d'une espèce et d'un genre nouveaux.

Cette Diatoméc, vue par la face valvaire, estévidemment un Pleurosigma, mais par la face connective, le frustule est courbé comme un Achnanthes, d'où le nom que M. Reinhard lui a donné. 
C'est une Diatomée marine, à frustules libres, avec un endochròme formé de deux plaques et un noyau placé contrc la valve concave. Sa taille est de 17 1/2 centièmes de millimètre. Elle est d'une grandc transparence, mais la disposition de ses stries n'est pas connue (1).

Cette formc, qui s'est trouvée en grandes quantités dans les récoltes de H. Mereschkowsky, établirait une transition des Pleurosigma aux Achnanthes, ou des NATiculÉES aux AcHNANTHÉEs.

\section{$\$$ 5. -- Berkeleya - Amphipleura.}

Les. Berkeleya constituent un petit genre créé autrcfois par Greville, et dans lequel M. H. Van Heurck ne cite que deux espèces : le Berkerleya micans, Lyngb. et le B. Dillwynii, Ag., qui vivent dans l'eau de mer.

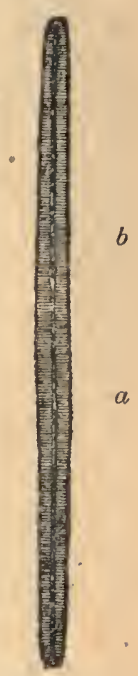

Fig. 263. - Berkeleya micans, Lyngb.

Le caractère le plus remarquable de cc genre est de présenter un nodule médian extrêmement allongé dans le sens longitudinal, s'effaçant dans la partie médiane et ne faisant saillie qu'à ses deux extrémités, de sorte qu'il parait dédoublé (2).

(1) L. Reinhard. - Les Bacillariacées de la mer Blanche. Bull. de la Soc. I. des Nat. de Moscou, et Journal de Micrographie, 1883.

(2) La figure ci-dessus du Berkeleya micans n'est pas très claire; néanmoins, on reconnaît, avec un peu d'attention et une loupe, les deux points extrêmes $a$ et $b$ du nodule médian dédoublè. 
Le Berkeleya micans est extrèmement long et mince, environ 20 fois plus long que large. Sa longueur varie de 8 a 9 cent. de millimètre. Il présente des stries fines et parallèles; 26 dans 1 cent. de mill.

Le Berkeleya Dilwynii est beaucoup plus petit, long de 3 centièmes de millim. environ, et elliptique, 4 fois moins large que long. Il présente 30 stries dans 1 cent: de mm., rayonnantes aux extrémités, parallèles au centre.

Amphipleura. - Les Amphipleura constituent un genre, établi paí Kützing en 1844, très pauvre en espèces, mais qui n'en est pas moins un des plus célèbres de la famille des Diatomées, gràce à l'Ampliipleura pellucida, Kz., l'une des plus intéressantes petites plantes qui soient au monde.

Nous ne croyons pas qu'il existe un seul organisme qui ait autant exercé la patience des micrographes, ait été l'objet de plus de recherches et de plus d'eflorts, qui ait plus contribué aux progrès de la construction du microscope et des objectifs, au perfectionnement des artifices d'observation et de la technique des préparations de Diatomées, que l'Amphipleura pellucida. Pendant de longues années, la plupart des diatomistes n'ont connu les stries de l'Amphipleura qüe par oui-dire, et nous ne craignons pas d'avancer que le jour où ils ont éprouvé leur plus grande joie est celui où, pour la première fois, ils ont vu apparaitre sous leur objectif ces stries délicates dont la résolution avait été si longtemps pour eux impossible et chimérique.

Aujourd'hui, avec les moyens perfectionnés que nous possédons, la résolution des stries transversales de cette petite Naviculée cs. beaucoup moins difficile, mais celle des stries longitudinales est encore très épineuse ; elle exige non seulement d'excellents instruments, mais de plus une grande habileté dans leur maniement; et, en somme, l'Amphipleura pellucida est restée le plus difficile de tous les tests. C'est elle qui forme encore le vingtième et dernier numéro du fameux test de Moller (1).

Ce petit genre, qui n'a longtemps compris qu'une espèce, celle dont nous venons de pąrler, n'en contient encore que deux, que nous sachions. Ses caractères génériques résident dans la disposition du

(1) Ler tests de Moller sont maintenant remplacés avec avantage par les magnifiques préparations-types de Ch. -J. Tempère, 168, rue Saint-Antoine, à Paris, préparations qui surpassent de beancoup celles de Moller, et coûtent infiniment moins cher: - Elles contiennent de 3 à 200 espèces. 
raphé, qui est droit sans nodule médian, mais se dilate vers les extrémités pour embrasser des nodules terminaux, très allongés et oeeupant souvent prés du einquième de la longucur de la valve.

Les valves sont fusiformes, très allongées, à extrémités atténuées et presque aiguës; elles sont de 9 à 11 fois aussi longues que larges et présentent, d'après Borseow, une ligne saillante longitudinale de ehaque eôté du raphé et un peu plus loin de ee raphé que du bord de la valve. Cette disposition n'est visible que quand on voit le frustule en eoupe optique transversale.

L'Amphipleura pellucidla est une espèce d'eau douce, assez eommune, dont la longueur varie de 8 à 1 כ̆ eentièmes de millimètre. Elle présente un système de stries transversales qui vont du bord jusqu'au raphé, qu'elles entament mème sur ses eôtés. Le nombre de.

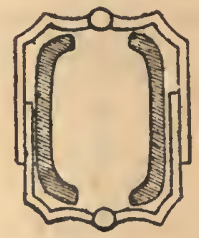

Fig. 264. - Coupe transversale schématique

de l'Amphipleura pellucida, Kz, d'après Borscow.

ces stries dans 1 eentième de millimètre est représenté par des chiffres assez différents, suivant les-divers auteurs. Nous en avons eompté 40 et M. Castraeane 52. Le Dr H. Van Heurck n'en indique que 37 en moyenne. C'est à ee chiffre qu'à notre avis on doit s'arrêter, car plus que tout autre il mérite eonfianee, II. Van IIcurek ayant (au mois de novembre 1884) réussi à résoudre en perles et à photographier l'Amphipleura pellucicla. Du reste, le chiffre donné par lui n'est qu'une moyenne et n'infirme pas absolument eelui qne nous avons trouvé. Quant à eelui de II. Castraeane, nous le croyons ecrtainement trop fort. Ce n'est pas, en effet, pareequ'elles sont très scrrées que ces stries sont difficiles à résoudre, mais paree qu'elles sont extrèmement délieates. D'autres Diatomécs ont une striation plus scrrée, quoique beaucoup plus faeile à voir.

Ces stries sont formées de perles dont l'alignement donne naissanee à des stries longitudinales. Celles-ei, d'après M. II. Van Heurck, ne sont pas plus serrées que les stries transversales, mais elles sont encore plus délicates et plus difficiles à résoudre. 
Ajoutons que la manière la plus facile de résoudre les stries de l'Amphipleura pellucida consiste à éclairer la Diatomée obliquement par la lumière solaire monochromatique, en faisant passer le

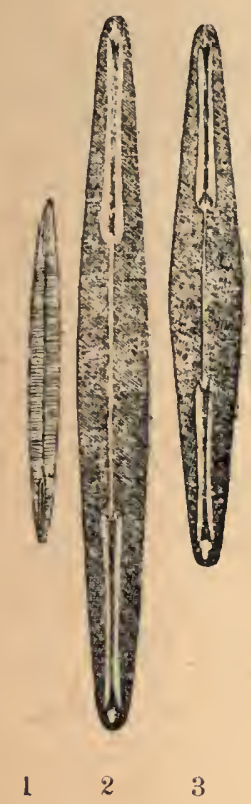

Fig. 265. - 1. Amphipleura pellucida, Kz.

2 et 3. Amphipleura Lindheimierii, Gr.

Var: Truani. H. v. H.

rayon de soleil à travers une petite cuve à faces parallèles contenant une solution de sulfate ou d'azotate de cuivre ammoniacal, comme nous l'avons indiqué plus haut. (Voir p. 17\%.)

M. Grunow a désigné sous le nom d'Amphipleura Lindheimerii une nouvelle espèce d'Amphipleura provenant du Brésil et du Texas. Elle est plus grande, mesurant de 16 à 22 centièmes de millimètre. Sa forme est à peu près la mème, sauf que les nodules terminaux sont plus robustes et encore plus longs, occupant chacun plus du quart, quelquefois près du tiers de la longueur de la valve. Celle-ci esı, du reste, moins étroite et plus naviculoïde que celle de l'A. pellucida, car elle n'est que de 6 à 8 fois aussi longue que large. Le raphé décrit à son milieu une petite ondulation latérale qui indique comme un rudiment de nodule médian. .

Cette espèce présenterait non seulement des stries transversales, 
mais un système de stries obliques inclinées à environ $45^{\circ}$ sur les premières, et dans un seul sens, c'est-à-dire d'un bord de la valve à l'autre en franchissant le raphé. La résolution de ces stries est difficile.

Enfin, M. A. Truan a trouvé, en 1883, dans des dépôts d'eaux ferrugineuses, dans la Trubia (Espagne), une dernière forme d'Amphipleura à laquelle il a donné le nom d'Amphipleura trubiana A. Tr. Elle présente les caractères de l'espèce brésilienne, A. Lindheimeriï, Gr.; M. H. Van Heurck la considère comme une varıété de cette dernière. Le savant diatomiste belge pense, d'ailleurs, que cet Amphipleura Lindheimerii, var, Truani, pourrait ètre la forme sporangiale de l'Amphipleura pellucida. 


\section{TABLE}

\section{ALPHABÉTIQUE DES MATIĖRES}

A

\begin{tabular}{|c|c|c|c|c|c|c|c|c|c|c|c|c|c|c|}
\hline & & & & & & & & & & & & & & \\
\hline HÉES & ${ }^{\circ}$ & - & $\bullet$ & & . & . & & & - & - & & & 192 & 21 \\
\hline hes. & 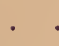 & . & & & & & & & & & & & 192 & 19 \\
\hline um, Bréb . & & 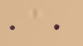 & & & & & & & & & & & & \\
\hline $\mathrm{ma}, \mathrm{I}$ & & . & - & . & & & & & . & & & & & 308 \\
\hline & . & . & . & & & & & & & & & & & \\
\hline , Ehb. & . & . & . & 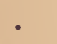 & & & & & & & & & & \\
\hline , Grev. & . & . & & & & & . & & & & & & & \\
\hline s, Ehb. & . & . & & & & & & & & & & & 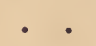 & $0 t$ \\
\hline Ehb. & . & . & & ${ }^{\circ}$ & & & & & & & & & & \\
\hline a, Kz. & & - & 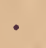 & 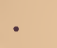 & & & & & $\cdot$ & & & & 195 & 1. \\
\hline , Ehb. & . & . & - & : & . & & . & & . & & & & & \\
\hline 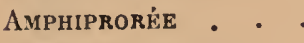 & . & . & - & - & - & & & & . & . & & & & 97 \\
\hline Ehb. & . & . & - & . & & & . & & & & & & & \\
\hline & - & . & . & - & . & & - & 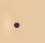 & . & - & & & 194 & \\
\hline . & . & . & & . & 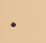 & & & & . & . & & & & 20 \\
\hline iatomé & ées . & . & . & . & . & 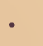 & - & - & 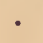 & . & & & - & 6 \\
\hline siti & & s parti & & & & & & & 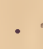 & & & & & \\
\hline & & - & - & . & - & & . & & . & . & & & . . & \\
\hline & & . & & & & & & & & & & & & \\
\hline & . & 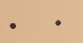 & - & $\bullet$ & 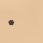 & • & . & & . & & & & & 0 \\
\hline & - & - & - & - & - & 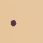 & 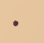 & 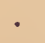 & . & & & & ${ }^{\circ}$ & \\
\hline & . & . $\quad \cdot$ & - & . & - & 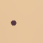 & - & • & · & . & & & & 20 \\
\hline Ehb. & . & - & . & . & & . & . & • & . & 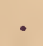 & & & • & 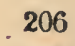 \\
\hline & - & . & - & - & - & - & . & - & - & 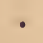 & & & & 20 \\
\hline Ehb. & . & - & . & - & - & 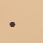 & & . & • & & & & & \\
\hline 10 & & & $\cdot$ & - & 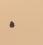 & & 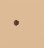 & & & & & & & \\
\hline
\end{tabular}




\section{$\mathrm{B}$}

Bacillaria, Gmel. . . . . . . . . . . . . . . . 197

Bucteriastrum, Lauder. . . . . . . . . . . . . . . 203

Baumes, vernis, résines . . . . . . . . . . . . 119

Berkeleya, Ehb. . . . . . . . . . . . . . 195,310

Biblarium, Ehb. . . . '. . . . . . . . . . . . . 202

Biddnlphia, Grun . . . . . . . . . . . . 204

Brddulphiées . . . . . . . . . . . . . . 204

Brebissonia, K\%. • . . . . . . . . . . . . . . . 195

Brightroellia, Ralf. . . . . . . . . . . . . . 207

\section{C}

Campylodiscus, Ehb. . . . . . . . . . . . . . . 197

Campylosira, Grun. . . . . . . . . . . . . . 199

Cerataulus, Ehb. . . . . . . . . . . . . . 204

Cestodiscus, Grev. . . . . . . . . . . . . 205

ChETOCÉRÉES . . . . . . . . . . . . . . . 203

Chatoceros, Ehb. . . . . . . . . . . . . . 203

Cladogramma, Fhb. . . . . . . . . . . . . . 203

Classifications . . . . . . . . . . . . . . . . . 187

Classification de M. H. L. Smith . . . . . . . . . 61, 188

Classification de M. P. Petit . . . . . . . . . . . . 189

Clavularia, Grev. . . . . . . . . . . . . . . . 199

Climacosphenia, Ehb. . . . . . . . . . . . . 201

Cocconeis, Ehb. . . . . . . . . . . 193, 211

Cocconema, Ehb. . . . . . . . . . . . . . 194, 226

Coccochromatickés. . . . . . . . . . . . . 192, 199

Condensateurs et appareils divers. . . . . . . . . . . . 165

Condensateur achromatique . . . . . . . . . 167, 171

Condensateur d'Abbé . . . . . . . . . . . . . 172

Condensateur de Dujardin : . . . . . . . . . . . . 166

Coscinodiscées . . • . . . . . . . . • . • . . 206

Coscinodiscus, Ehb. • . . . . . . . . . . . . 207

Craspedodiscus, Ehb. . . . . . . . . . . . . . . . 207

Craspedoporus, Ehb. . . . . . . . . . . . . . 205

Creswellia, Grev. • . . . . . . . . • . . . 207

Cyclotella, Kz. . . . . . . . . . . . . . . : . 208

Cyclophora, Cast. . . . . . . . . . . . 193, 217

Cylindrotheca, Gr. . . . . . . . . . . . . 203

Cymatopleura, W. Sm. . . . . . . . . . . . . 197.

Cymatosira, Gr. • . . . . . . . . . . . . . . . 199

ऽymbella, Ag. . . . . . . . . . . . . . . . . . 194, 239 
CYMBellées .

Cymbosira, Kz.

Debya, Paut.

Déduplication des Diatomées.

Denticula, Kz.

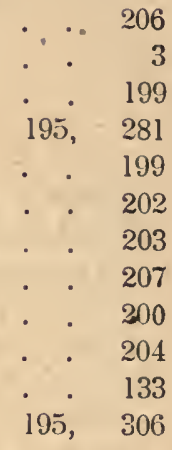

Diadesmis, $\mathrm{Kz}$.

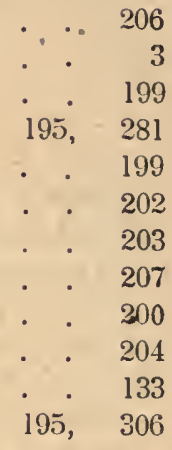

Diatoma, Ag.

Diatomella, Grev.

Dicladia, Ehb.

Dictyopyxis, Ehb.

Dimeregramma, Ralfs .

Ditylum, Bail.

Doigt mécanique

199

202

203

207

200

204

Donkinia, Ralfs. 306

E

Eclairage.

Eclairage d'Abbé

172

Eclairage électrique.

178

Eclairage solaire monochromatique . . . . . . . . . . . . 175

Encyonema, Kz.

$194, \quad 229$

Epithemia, Bréb.

$194, \quad 233$

Eucampia, Ehb.

204

Eunotia, Ehb.

Eunotí́es

199

198

Eunotogramma, Weiss. . . . . . . . . . . . . . 205

Euodia, Bail.

205

Eupodiscées.

204

Eupodiscus

205

F

Fragilaria, Lyngb . . . . . . . . . . . . . . . . 199

FragiLARIÉES

G

Gaillonellá, Bory . . . . . . . . . . . . . . 208

Gaillonellées . 
Gephyria, Arn.

Pages

Glyphodesmis, Grev. . . . . . . . . . . . . . 200

Gomphogramma, Braun . . . . . . . . . . . . 202

Gomphonema, Ag. . . . . . . . . . . . . . . 194, 219

GoMpHonÉuḱEs .

194, 218

Goniothecium, Ehb.

203

Grammatophora, Ehb.

202

$\mathrm{H}$

Halionyx, Ehb.

Heliopelta, Ehb.

HÉLIOPELTÉES

206

205

204

Hemiaulus, Ehb.

205

Hemidiscus, Wall

203

Hercotheca, Ehb.

207

199

Himantidium, Ehb.

Histoire naturelle des Diatomées . . . . . . . . . . . . 20

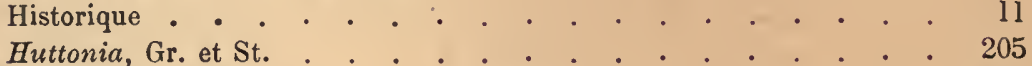

Hyalodiscus, Ehb. . . . . . . . . . . . . . . 208

Hyalosira, $\mathrm{Kz}$.

202

\section{I}

Instruments et produits pour le montage . . . . . . . . 118

Introduction .

Isthmia, Ag. 204

$\checkmark$

Janischia, Gr. . . . . . . . . . . . . . 204

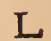

Lipidodiscus, 0. Witt. . . . . . . . . . . . 206

Licmophora, Ag. . . . . . . . . . . . . . . . 201

LicMophóḱes . . . . . . . . . . . . . . 201

Liquides conservateurs, . . . . . . . . . . . . . . 122 
Mástogloia, Thw.

Pages

Meridion, Ag.

$195, \quad 280$

Microscopes

Microtomes

181

Milieux à haut indice

121

Mode de végétation des Diatomées

Monopsia, Grov. et St.

205

Montage des Diatomées

118

Montage à sec . .

122

Montage dans les baumes.

124

Montage dans les liquides.

127

Mouvements des Diatomées

Moyens d'étude . . • . . . . . . . . . . . . 138

Multiplication des Diatomées . . . . . . . . . . . . . 36

Multiplication par conjugaison . . . . . . . . . . . . . 4 43

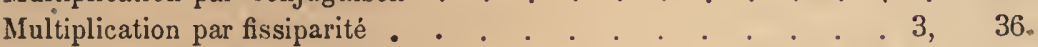

Multiplication par rajeunissement. . . . . . . . . . . 43

\section{$\mathbf{N}$}

Navicula, Bory . . . . . . . . . . . . . 195, 245

NAviculḱes . . . . . . . . . . . . . . 195, 243

Nettoyage des Diatomées . . . . . . . . . . . 36

Nitzschia. Hass. . . . . . . . . . . . . . . 197

NitZSCHIÉES . . . • . . . . . . . . . . 196

Nombre des stries . . . . . . . . . . . . . . 82

Noyau . . . . . . . . . . . . . . . . 89

O

Objectifs . . . . . . . . . . . . . . . 148

Omphalopsis, Grev . . . . . . . . . . . . 200

Opephora, P. P. . . . . . . . . . . . . . 201

\section{$\mathrm{P}$}

Pantocsekia, Grun . . . . . . . . . . . . 208

Periptera, Ehb. . . . . . . . . . . . . 203

Peronia, Bréb. . . . . . . . . . . . . 201

Pinnularia, Ehb. . . . . . . . . . . . . 246 
Placochromaticées.

Plagiogramma, -Grev.

\section{Plagiogrammées}

Pleurosigma, W. Sm.

Podocystis, Kz.

Podosirc, Ehb.

Podosphenia, Ehb. . . . . . . . . . . . . . 201

Polymyxus, Bail . . . . . . . . . . . . . . . 206

Porodiscus, Grev. . . . . . . . . . . . . . . . 207

Porpeia, Bail . . . . . . . . . . . . . . . 204

Préparations systématiques . . . . . . . . . . . 128

Préparations systématiques (procédé Rataboul). . . . . . . . 131

Préparations dans la naphtaline monobromée . . . . . . . . 136

Principaux gisements des Diatomées . . . . . . . . . . 96

Procédé de nettoyage par l'acide nitrique . . ^. . . . . . 107

Procédé de nettoyage par l'acide sulfurique et le chlorate de potasse . 109

Procédé de nettoyage par le permanganale de potasse et l'acide chlorhydrique . . . . . . . . . . . . . . . . 110

Procédé de nettoyage pour les dépôts marins (M. Kitton) . . . . 111

Procédé de nettoyage pour les guanos, les sondages marins et les fossiles. M. Kitton)

Procédé de nettoyage pour les guanos et les vases marines. (M. J. Brun)

Pseudauliscus, Leud. F.

Pseudo-Eunotia, Ehb.

Pterotheca, Grev.

Pyxidicula, Ehb.

R

Raphé, pseudo-raphé . . . . . . . . . . . . . 59

Raphoneis, Ehb. . . . . . . . . . . . . . . . . 199

Recherche et récolte des Diatomées . . . . . . . . . . 93

Récolte . . . . . . . . . . . . . . . . . . . 99

Reproduction des Diatomées . . . . . . . . . . . . . 7

Reproduction par conjugaison. . . . .

Réviviscence des Diatomées . . . . . . . . . . . . . . . . . 55

Rhabdonema, Ehb. . . . . . . . . . . . . . . . . 202

Rhiэosolenia, Ehb. . . . . . . . . . . . . . 203

Rhizosoleniḱrs, Ehb. • • . . . . . . . . . . . . 202

Rhoïconeis, Grun . . . . . . . . . . . . . . . 193

Rhoïcosigma. . . . . . . . . . . . . . . . 306

Rhoïcosphenia, Grun . . . . . . . . . . . . 194, 219

Roperià, Grun . . . . . . . . . . . . . . . 205 
$\mathrm{S}$

Sceptroneis, Ehb.

Pages

Schizonema, Kz.

Scoliopleura, Grun.

201

Skeletonema, Grun.

$\begin{array}{ll}195, & 278 \\ 195, & 287\end{array}$

Stauroneis, Ehb.

207

Staurosira, Ehb.

282

198

Stephanodiscus, Ehb

207

Stephanogonia, Ehb.

203

Stephanopyxis, Ehb.

207

Stictodesmis, Grev.

195

Stigmaphora, Wall.

195

207

Strangulonema, Grev.

202

Striatella, Ag.

79

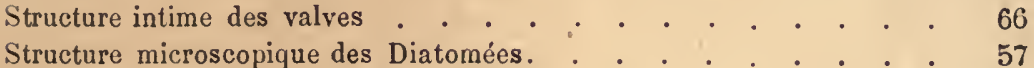

Surirella, Turp.

Surirellées.

Syndendrium, Ehb.

197

203

Synedra, Ehb.

SYNÉdRÉES

Syringidium, Ehb.

198

198

Systephania, Ehb.

203

$\cdot 203$

Tabellaria; Ehr.

202

TABELlariées

201

Technique des coupes.

181

Technique des Diatomées . . . . . . . . . . . 105

Terebraria, Grev.

- 199

Terpsinö, Ehb.

Tesselln, Ehb.

202

Tetracyclus, Ralfs. . . . . . . . . . . . . . 202

Thalassiothrix, Ehb. . . . . . . . . . . . . . . 198

Toxarium, Bail.

Toxonidea, Donk.

195, 308

Trachysphenia, P. P.

Trachysphéniées

201

200

Triage des Diatomées (M. Kitton)

117

Triage des Diatomées (M. H. L. Smith). 
Pages

Truania, Pant. . . . . . . . . . . . . 206

Tryblionella, W. Sm. . . . . . . . . . . . . . . 197

$$
\mathrm{V}
$$

Valves . . . . . . . . . . . . . . . . . 57

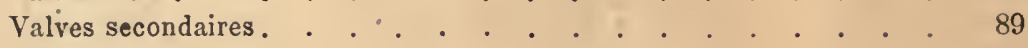

Vanheurckia, Bréb. . . . . . . . . . . . . . . . 277

Vernis, baumes; résines . . . . . . . . . . . . . 119

$\mathrm{X}$

XANTHIOPYXIDÉES . . . . . . . . . . . . . 207

Xanthiopyxis, Ehb. . . . . . . . . . . . . . 207 



